\title{
Biomechanical Optimization of the Angle and Position for Surgical Implantation of a Straight Short Stem Hip Implant
}

\author{
by
}

\author{
Gillian E. Cook
}

\begin{abstract}
A thesis submitted in conformity with the requirements for the degree of Master of Health Science in Clinical Engineering

Institute of Biomaterials and Biomedical Engineering University of Toronto
\end{abstract}




\title{
Biomechanical Optimization of the Angle and Position for Surgical Implantation of a Straight Short Stem Hip Implant
}

\author{
Gillian E. Cook \\ Master of Health Science in Clinical Engineering \\ Institute of Biomaterials and Biomedical Engineering \\ University of Toronto \\ 2014
}

\section{Abstract}

Conservative hip implants preserve healthy bone for revision surgeries and improve physiological loading; however, they have few supporting biomechanical data. This thesis determined the $3 \mathrm{D}$ orientation of a straight short stem hip implant within the proximal femur that would yield a stress distribution most similar to an intact femur. Synthetic femurs were implanted with a stem in one of seven maximum angles or positions and axially loaded, with resultant strain values used to validate a finite element model. Design of experiments was used to analyze the range of potential implant orientations under three gait cycle loading conditions. A global optimal orientation of $9.14^{\circ}$ valgus, $2.49^{\circ}$ anteversion, $0.48 \mathrm{~mm}$ posterior position, and $0.23 \mathrm{~mm}$ inferior position yielded optimal stress distributions across the range of the gait cycle. Orthopaedic surgeons should therefore implant straight short stem hip implants in valgus up to $10^{\circ}$ with an otherwise neutral position and version for maximal physiological loading. 


\section{Acknowledgments}

There are many people who contributed to the success of this thesis, and I could not have done it without each and every one of them.

Firstly, I would like to thank my exceptional supervisor, Dr. Emil Schemitsch, for his unwavering guidance and support these past two years. I would also like to thank my outstanding committee members, Dr. Cari Whyne and Dr. John Davies, for continually encouraging me to do more and be better.

I am eternally grateful to Dr. Radovan Zdero for always being there when I had questions or needed advice.

I offer my sincere thanks to Zachary Morison, Dr. Mina Aziz, Elnaz Ajami, Suraj Shah and Bruce Nicayenzi for their invaluable technical guidance and aid.

To Saeid Samiezadeh: you taught me how to finite element model. What more can I say?

I must extend my gratitude to Dr. Julie Audet and Hamid Ebrahimi for their profound statistical knowledge.

I would like to acknowledge Mr. and Mrs. Frank and Barbara Milligan for their financial support of this thesis.

Finally, I would like to thank my family. Mom, you were always there when I needed someone to talk to (or complain to), and I'm indebted to you for all of your hours spent profreading. Family, you suffered me in the lows and celebrated with me in the highs, and I could not have completed this without you. Thank you. 


\section{Table of Contents}

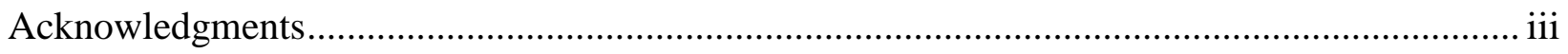

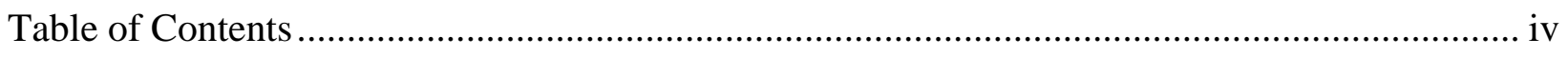

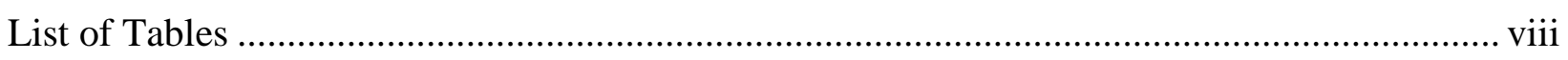

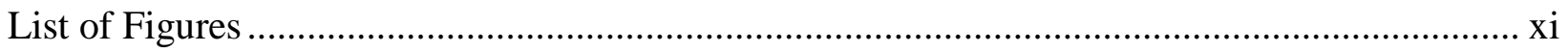

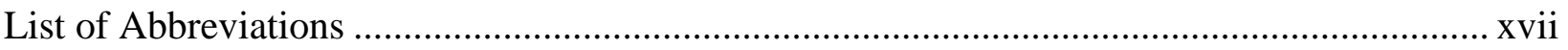

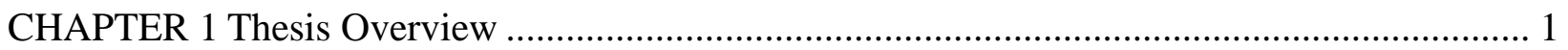

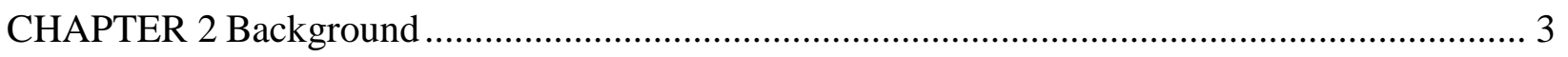

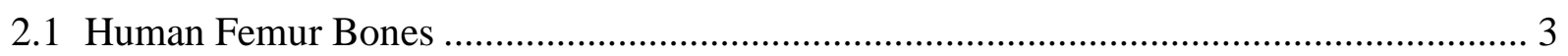

2.1.1 Composition and Structure …………………….......................................... 3

2.1.2 Biomechanical Properties ............................................................................. 3

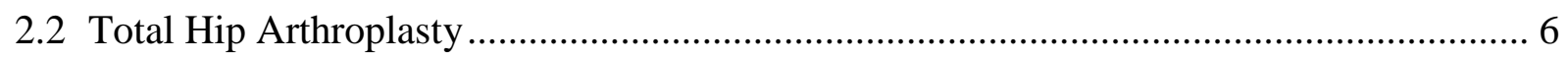

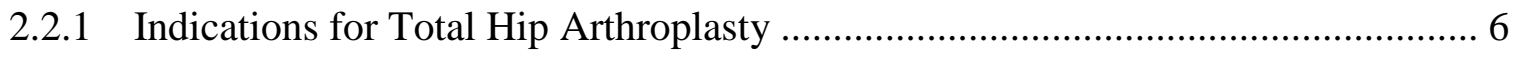

2.2.2 Hip Replacement Demographics ................................................................ 7

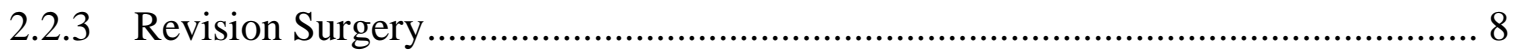

2.2.4 Cemented and Cementless Implants .................................................................. 8

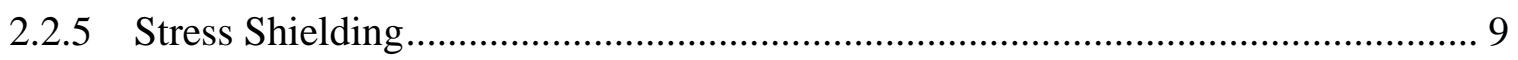

2.2.6 Gruen Zones ............................................................................................... 10

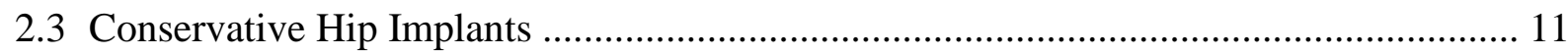

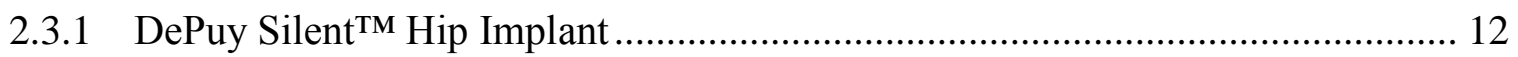

2.3.2 Examination of Orientation.......................................................................... 14

CHAPTER 3 Thesis Objectives and Hypothesis ………............................................................ 15

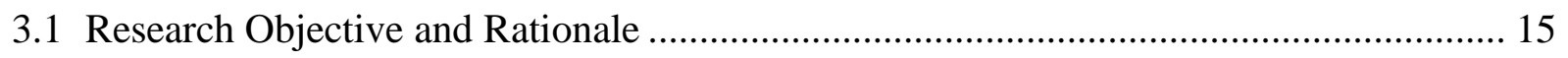

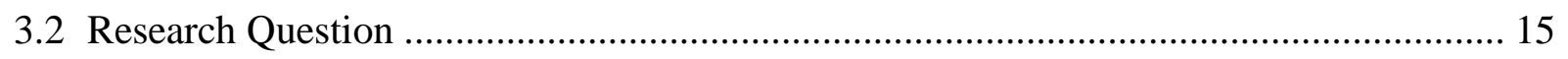

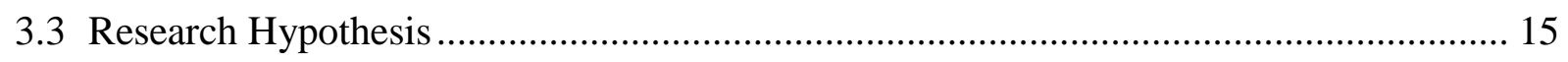

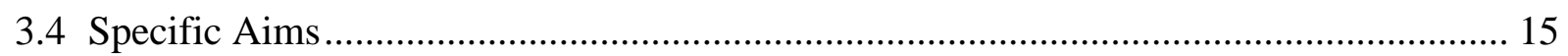




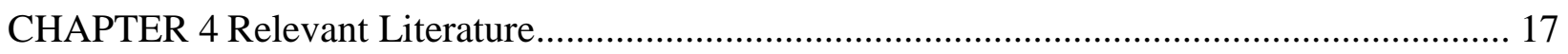

4.1 Conservative Hip Implant Reviews ............................................................................ 17

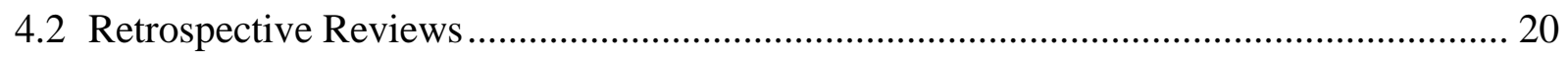

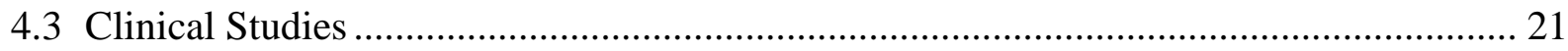

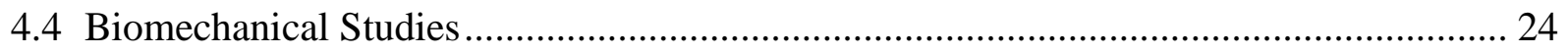

4.4.1 Finite Element Modelling Studies .................................................................. 24

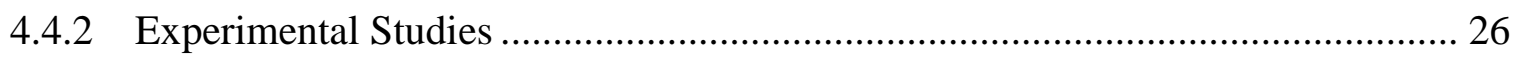

4.4.3 Implant Orientation Studies ......................................................................... 27

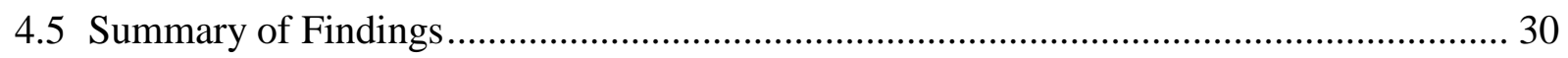

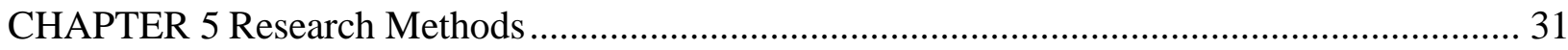

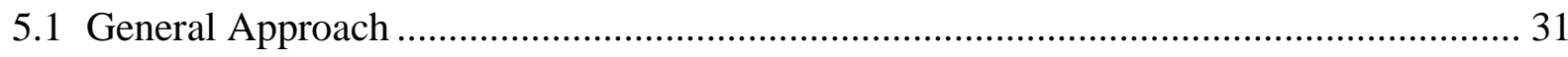

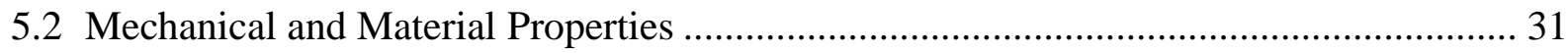

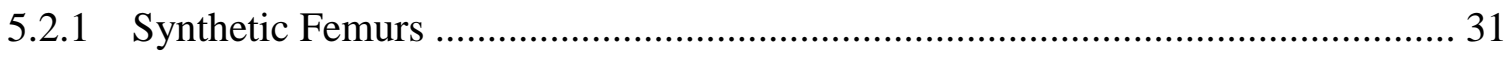

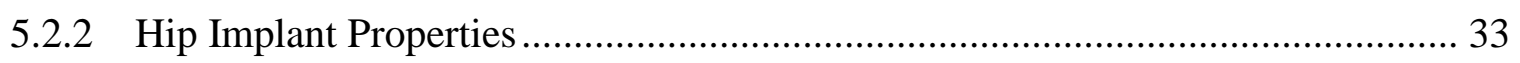

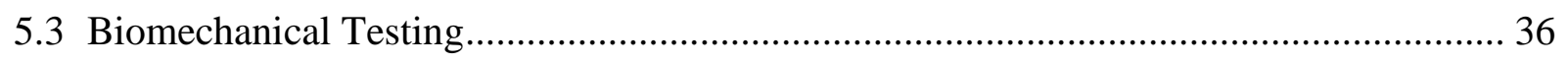

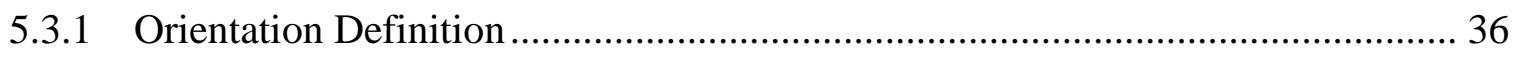

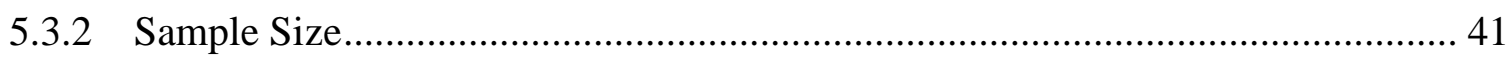

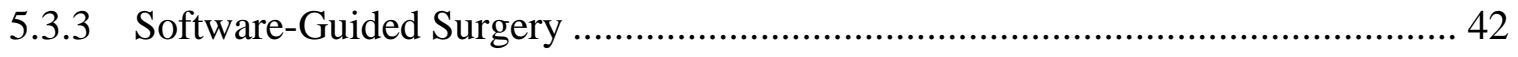

5.3.4 Silent Stem Implantation............................................................................... 46

5.3.5 Orientation Verification ............................................................................. 49

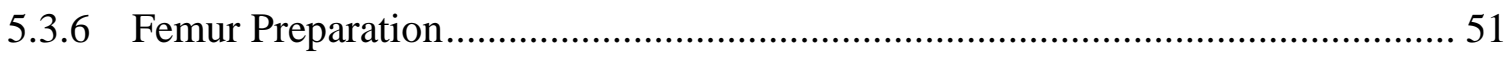

5.3.7 Biomechanical Testing.................................................................................... 54

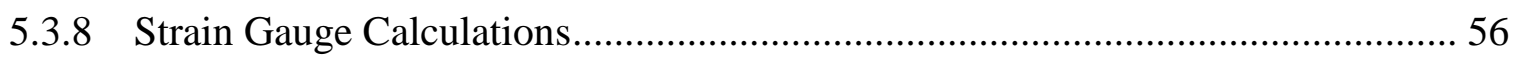

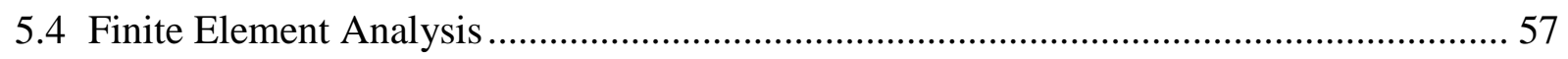

5.4.1 Generation of Solid Models ......................................................................... 57

5.4.2 ANSYS Workbench for Finite Element Analysis ................................................ 59 


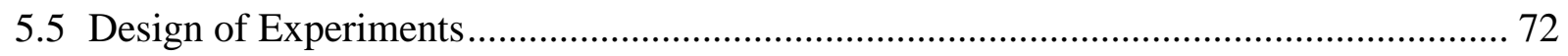

5.5.1 OFAT versus Factorial Design ....................................................................... 73

5.5.2 Response Surface Methodology Designs........................................................... 74

5.5.3 JMP for Design of Experiments.................................................................. 75

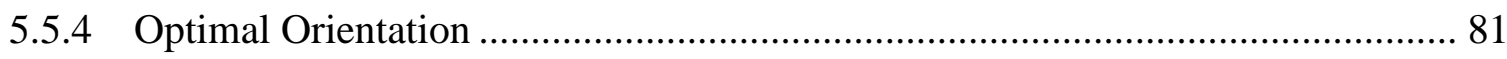

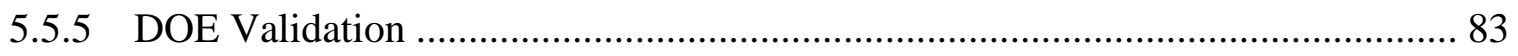

5.5.6 Global Optimal Orientation Verification ………………….................................. 83

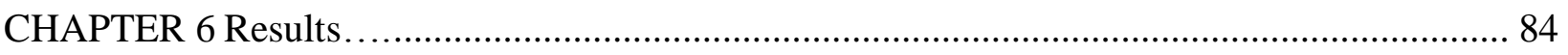

6.1 Verification of Implant Orientation .............................................................................. 84

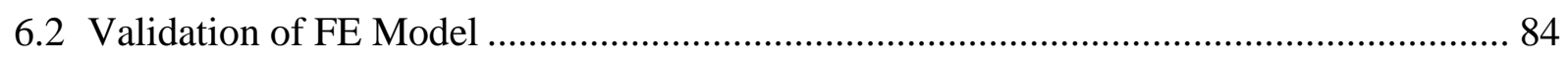

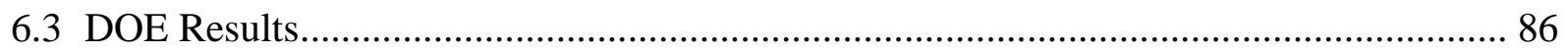

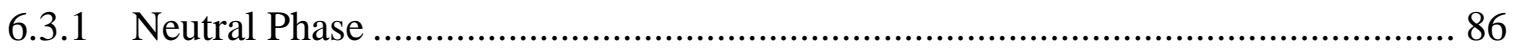

6.3.2 Extension Phase …………………………………………………………..... 104

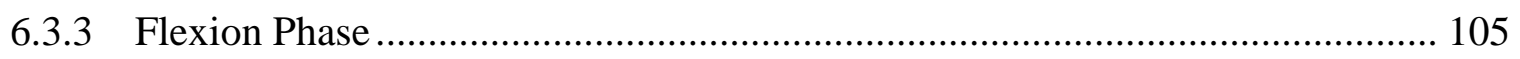

6.3.4 Global Optimal Orientation ......................................................................... 106

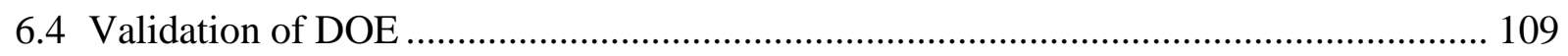

6.5 Validation of Global Optimal Orientation .................................................................. 111

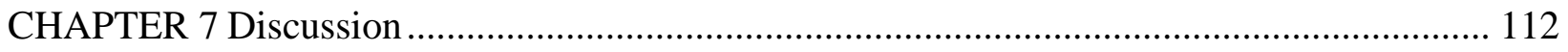

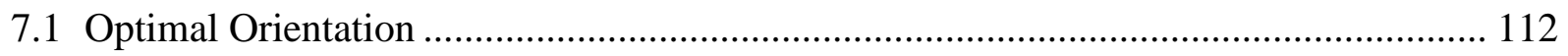

7.1.1 Common Significant Terms ........................................................................... 113

7.1.2 Region- and Phase-Specific Significant Terms ................................................. 114

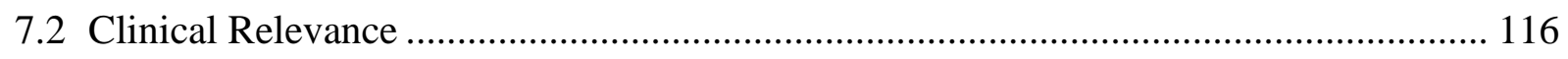

7.3 Study Limitations and Future Directions ....................................................................... 118

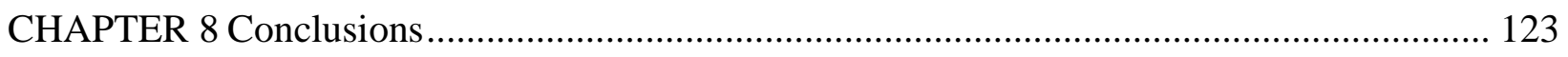

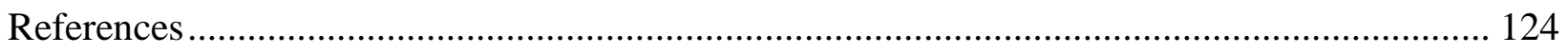

Appendix A: DOE Outcome Responses ..................................................................................... 136 
Appendix B: Weighted Averages for Each Gait Phase

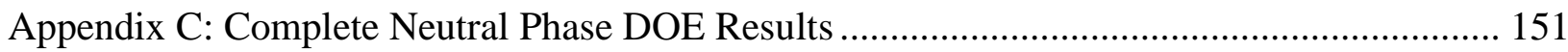

Appendix D: Complete Extension Phase DOE Results .............................................................. 173

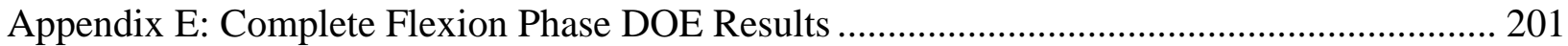




\section{List of Tables}

Table 1: Average mechanical and material properties for human femoral bone ${ }^{2,7} \ldots$ 5

Table 2: Mechanical and material properties for synthetic femur bones ${ }^{63}$ 32

Table 3: Material properties of the ceramic femoral head and titanium alloy stem ${ }^{68-70} \ldots \ldots \ldots \ldots . . . . .35$

Table 4: Planned angles and positions in the A/P and lateral planes for each of the 6 maximum angles and positions, and neutral

Table 5: List of the Young's modulus and Poisson's ratio used for each material in the ANSYS Workbench simulations 60

Table 6: DOE factors and corresponding maximum levels, as achieved in the biomechanical tests .73

Table 7: CCD factor levels with corresponding coded names and values 76

Table 8: All of the runs specified by JMP given the chosen inputs, with a pattern indicating the composition of each run: "+" for high setting, “-” for low setting, "A" high axial, "a" low axial, " 0 " for centre point ${ }^{111}$ 77

Table 9: Overview of each response selection approach and the method used to generate the employed input value 80

Table 10: Summary of the reduced Proximal Region stress model, sorted by significance, and indicating each response measure with its corresponding model terms, coefficients, and $p$-values. Significant terms have been marked with an asterisk $\left(^{*}\right)$ and bolded 87

Table 11: Summary of the reduced Proximal Region \% of intact femur model, sorted by significance, and indicating each response measure with its corresponding model terms, coefficients, and $p$-values. Significant terms have been marked with an asterisk $(*)$ and bolded.90

Table 12: Summary of the reduced regional stress model, sorted by significance, and indicating each response measure with its corresponding model terms, coefficients, and $p$-values. Significant terms have been marked with an asterisk (*) and bolded 92 
Table 13: Summary of the reduced regional \% of intact model, sorted by significance, and indicating each response measure with its corresponding model terms, coefficients, and $p$-values. Significant terms have been marked with an asterisk $(*)$ and bolded.

Table 14: Summary of the reduced regional $90^{\text {th }}$ percentile stress model, sorted by significance, and indicating each response measure with its corresponding model terms, coefficients, and $p$ values. Significant terms have been marked with an asterisk $(*)$ and bolded. 98

Table 15: Summary of the reduced regional $90^{\text {th }}$ percentile $\%$ of intact stress model, sorted by significance, and indicating each response measure with its corresponding model terms, coefficients, and $p$-values. Significant terms have been marked with an asterisk $(*)$ and bolded.

Table 16: Summary of common significant neutral terms and their corresponding effects, in addition to the region which was most significantly affected by the term 102

Table 17: Compilation of all optimal orientations generated by each neutral phase response model, specifying the weighted average values for each factor and the factor range 102

Table 18: Summary of common significant extension terms and their corresponding effects, in addition to the region which was most significantly affected by the term 104

Table 19: Compilation of all optimal orientations generated by each extension phase response model, specifying the weighted average values for each factor and the factor range 105

Table 20: Summary of common significant flexion terms and their corresponding effects, in addition to the region which was most significantly affected by the term 106

Table 21: Compilation of all optimal orientations generated by each flexion phase response model, specifying the weighted average values for each factor and the factor range 106

Table 22: Global optimal orientation, including weighted average values for each factor and the total acceptable factor range 107

Table 23: Response outcome measures for each Region of Interest across all gait phase optimal orientations, including the global optimal orientation, for loading under $1000 \mathrm{~N}$. Bolded and 
starred terms indicate responses that are more similar to the intact femur value than any other response outcome measure for that particular DOE ...................................................... 109 


\section{List of Figures}

Figure 1: Load versus deformation plot for bone, indicating the elastic and yield regions ${ }^{1} \ldots \ldots . . .4$

Figure 2: Stress-strain curve for bone, demonstrating the influence of strain rate on behaviour ${ }^{2,5}$ 5

Figure 3: a) Anatomy of a healthy hip joint; b) Outcomes of an osteoarthritic hip joint ${ }^{12} \ldots \ldots \ldots . . .7$

Figure 4: Radiographs of the right hip: a) before implantation; b) immediately postoperatively; c) 7.5 years after surgery, with severe stress shielding noted ${ }^{35}$. 10

Figure 5: Diagram depicting the seven Gruen Zones 39 11

Figure 6: A short stem hip implant with a lateral flare for metaphyseal fixation ${ }^{20}$ 12

Figure 7: Illustration of the DePuy Silent Hip (DePuy, Leeds, UK) ${ }^{17}$ 13

Figure 8: Silent Hip implanted in the femoral neck, demonstrating high neck cut ${ }^{17}$ 14

Figure 9: a) Short modular femoral stem (Smith \& Nephew, Inc., Memphis, TN, USA) ${ }^{57}$; b) Mayo conservative implant double wedge stem with proximal coating (Zimmer, Rochester, MN, USA) ${ }^{20}$ 18

Figure 10: a) Proxima Hip (DePuy, Leeds, UK) ${ }^{57}$; b) Birmingham mid-head resection (BMHR) (Smith \& Nephew, Marl, Germany) ${ }^{58}$

Figure 11: Silent Hip (DePuy, Leeds, UK) ${ }^{17}$

Figure 12: Radiograph of an implanted Type A short stem hip implant (right hip) and a Type B ultra-short stem implant (left hip), with a numerical depiction of the 5 modified Gruen zones for short stem implants 43

Figure 13: Radiograph of an implanted Gothenburg osseointegrated titanium (GOT) arthroplasty 41 23

Figure 14: Pre-remodeling and post-remodeling shear stresses in the long and short-stemmed reconstructions, illustrating the implant design and short stem model ${ }^{61}$ 24 
Figure 15: Depiction of compressive strain and strain shielding in the intact and reconstructed femur models 47

Figure 16: Depiction of the changes in the absolute value of the major principal strains (in \% of the intact femur value) after implantation of the Alloclassic (left), OPTAN (middle), and CUT (right) ${ }^{31}$

Figure 17: Depiction of cortical strain energy in each Gruen zone for all solid models under walking and stair climbing loads. Intact refers to the intact femur model, Ref is the reference implanted model, Med corresponds to the medialised model, and AV is the anteverted model ${ }^{62}$ 29

Figure 18: Strain distributions for a single femur model in the: a) intact femur, b) resurfaced in varus, c) resurfaced in neutral, d) resurfaced in valgus ${ }^{54}$ 30

Figure 19: Large fourth generation composite femur and femur cross-section (Model \#3406, Pacific Research Laboratories, Vashon, Washington, USA). Sizing is as follows: a) $485 \mathrm{~mm}, \mathrm{~b}$ ) $52 \mathrm{~mm}, \mathrm{c}) 37 \mathrm{~mm}, \mathrm{~d}) 120^{\circ}$, e) $\left.\left.32 \mathrm{~mm}, \mathrm{f}\right) 93 \mathrm{~mm}, \mathrm{~g}\right) 16 \mathrm{~mm}$ canal $^{63}$ 32

Figure 20: SEM of the Silent Hip stem, depicting the sintered calcium phosphate bead coating at x30 magnification 33

Figure 21: EDX of the stem neck region, illustrating the Ti-Al-V composition of the stem ....... 34

Figure 22: EDX of the stem body region, indicating the composition of the calcium phosphate beaded coating 34

Figure 23: Expanded scale EDX of the ceramic femoral head, indicating the aluminum oxide matrix composition 35

Figure 24: A/P view of the Silent stem neutrally implanted within the femur, where the solid red line indicates the long axis of the femur and the dashed line represents the implant axis at a CCD angle of $120^{\circ}$ 37 
Figure 25: Lateral view of the Silent stem neutrally implanted within the femur, where its neutral angle and position are displayed through its central location between lines tangent to the cortical bone. 37

Figure 26: A/P view of a Silent stem implanted in $130^{\circ}$ valgus 38

Figure 27: A/P view of a Silent stem implanted in $110^{\circ}$ varus..... 39

Figure 28: Lateral view of a Silent stem implanted in $10^{\circ}$ anteversion 39

Figure 29: Lateral view of a Silent stem implanted in $2 \mathrm{~mm}$ anterior 40

Figure 30: Lateral view of a Silent stem implanted in $2 \mathrm{~mm}$ posterior 40

Figure 31: A/P view of a Silent stem implanted in $4 \mathrm{~mm}$ inferior.

Figure 32: Depiction of the Birmingham Hip Resurfacing (BHR) implant (Smith \& Nephew, Marl, Germany) ${ }^{75}$

Figure 33: Depiction of an optical array of calibration markers attached to the intact femur 43

Figure 34: Screenshot of the surgical planning screen provided by the BrainLAB Hip Essential software 44

Figure 35: Image of the registered femur undergoing guidewire placement using a drill and marker-equipped drill guide. 45

Figure 36: Screenshot of the planned and achieved surgical paths for a neutrally aligned guidewire 46

Figure 37: Reamers included in the Silent Hip surgical technique 47

Figure 38: Silent stem placement using the implant impactor instrument, indicating the final seating height and the $3 \mathrm{~mm}$ distal gap 48

Figure 39: Model of the intact femur, where the solid red lines indicate the respective resection lines and the dashed line indicates the longitudinal axis of the femur 
Figure 40: A/P view of a neutrally implanted femur, indicating the neutral $120^{\circ} \mathrm{CCD}$ angle formed by the long axis of the femur and the implant 50

Figure 41: Lateral view of a neutrally implanted femur, depicting the modified Resubal method for determination of implant neutrality: a) tangent circles drawn on femoral neck; b) line drawn through circle centre-points, and distance to axis of implant measured 51

Figure 42: Synthetic femur located in a steel cube in preparation for potting, indicating the desired $7^{\circ}$ of adduction 52

Figure 43: Gauged femur, indicating the location of the medial linear gauges (1 and 2), lateral linear gauges $(3,4$, and 5$)$, and the rosette gauge 53

Figure 44: Experimental set-up, depicting the femur locked into a neutral orientation within the Instron machine and attached to the Cronos data acquisition system (DAS) 55

Figure 45: Silent Hip model fitted to the femoral head, indicating location of extra bodies in blue for eventual Boolean operations in ANSYS 58

Figure 46: CAD model of the implanted femur assembly with the Silent stem in neutral .......... 59

Figure 47: Screenshot of ANSYS DesignModeler, depicting the tree outline with each modification step. 62

Figure 48: Comparison of linear neutral model with load applied directly to femoral head (left) versus a nonlinear neutral model with contact between head and load plate set to frictionless (right), equivalently scaled in von Mises stresses (MPa) for comparison purposes 64

Figure 49: Depiction of a 10-node quadratic tetrahedron ${ }^{95}$ 65

Figure 50: Proximal cross-section of the mesh used for this study, depicting the finer mesh and areas of refinement 66

Figure 51: Screenshot of the final mesh used in this study..... 67

Figure 52: Proximal Region of interest selected based on a global coordinate system location, where the pink dots indicate underlying nodes..... 69 
Figure 53: Inferior Neck Region, depicting selected nodes

Figure 54: Superior Neck Region, depicting selected nodes 70

Figure 55: Lateral Region, depicting selected nodes 71

Figure 56: Depiction of a black-box system with variable inputs and corresponding responses ${ }^{107}$ 72

Figure 57: Two-level factorial design depicting the contents of each run, and the volume covered by the design 108 73

Figure 58: Depiction of a two-level CCD design space ${ }^{107}$ 74

Figure 59: Comparison of the three types of CCD, indicating factorial ranges (blue) and star points (pink) 109 75

Figure 60: Colour map depicting the aliasing of effects, where a blue square indicates no correlation and a red square indicates complete aliasing 78

Figure 61: Screenshot of the desirability tool, where the lower graphs indicate the desirability response curve for each factor and the corresponding degree of desirability on the $y$-axis for each of the given factor settings, indicated by the numbers in red 82

Figure 62: Correlation of all measured experimental and corresponding FEM strains including a line of best fit with $95 \%$ confidence limits. The equation of fit is $y=30.52+1.18 x$, with a

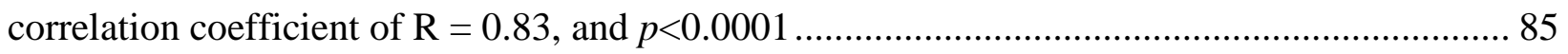

Figure 63: Residual plot of measured versus predicted strains......................................... 85

Figure 64: Bar chart of FEM and average experimental stiffness values for each implant orientation 86

Figure 65: Output of the desirability tool for stress response, indicating ideal factor settings along the bottom row, with corresponding stress responses and CI along the left. Starting from the top row and going down, intact femur values for this optimization were $5.13 \mathrm{MPa}, 3 \mathrm{MPa}$, and 9.19MPa. 88 
Figure 66: Output of the desirability tool for \% of intact, indicating ideal factor settings along the bottom row, with corresponding stress responses and CI along the left. All intact femur values for this optimization corresponded to 100

Figure 67: Output of the desirability tool for mean stress, indicating ideal factor settings along the bottom row, with corresponding stress responses and CI along the left. Starting from the top row and going down, the intact femur values for this optimization were 9.19MPa, 6.11 $\mathrm{MPa}$, 4.06MPa and 5.13MPa 93

Figure 68: Output of the desirability tool for percent of intact stress, indicating ideal factor settings along the bottom row, with corresponding stress responses and CI along the left. All intact femur values for this optimization corresponded to 100 . 96

Figure 69: Output of the desirability tool for $90^{\text {th }}$ percentile stress, indicating ideal factor settings along the bottom row, with corresponding stress responses and CI along the left. Starting from the top row and going down, the intact femur values for this optimization were 9.19MPa, 13.61 $\mathrm{MPa}, 9.66 \mathrm{MPa}$ and $5.88 \mathrm{MPa}$ 99

Figure 70: Output of the desirability tool for $90^{\text {th }}$ percentile $\%$ of intact femur stress, indicating ideal factor settings along the bottom row, with corresponding stress responses and CI along the left. All intact femur values for this optimization corresponded to 100 101

Figure 71: FE model of the intact femur loaded at 3000N (top), and the neutral phase optimally implanted femur loaded at 3000N (bottom), equivalently scaled in von Mises stresses (MPa) for comparison purposes. 103

Figure 72: FE model of the intact femur loaded at 3000N (top), and the global optimally implanted femur loaded at 3000N (bottom), equivalently scaled in von Mises stresses (MPa) for comparison purposes 108 


\section{List of Abbreviations}

ANOVA: Analysis of Variance

A/P: Anteroposterior

BHR: Birmingham Hip Resurfacing

BMD: Bone Mineral Density

BMHR: Birmingham Mid-Head Resection

BW: Body Weight

CAD: Computer-Aided Design

CCD Angle: Caput-Collum-Diaphyseal Angle

CCD: Central Composite Design

CI: Confidence Interval

CT: Computed Tomography

DAS: Data Acquisition System

DEXA: Dual-Energy X-Ray Absorptiometry

DICOM: Digital Imaging and Communications in Medicine

DOE: Design of Experiments

DOF: Degrees of Freedom

EDX: Energy-Dispersive X-Ray Spectroscopy

FE: Finite Element

FEA: Finite Element Analysis 
FEM: Finite Element Method

HA: Hydroxyapatite

HHS: Harris Hip Score

IR: Infrared

OA: Osteoarthritis

OFAT: One-Factor-at-a-Time

PMMA: Polymethylmethacrylate

RMSE: Root-Mean-Square Error

RSA: Radiostereometric Analysis

RSD: Relative Standard Deviation

RSM: Response Surface Methodology

SE: Standard Error

SEM: Scanning Electron Microscopy

SNA: Stem-Neck Angle

SE: Standard Error

THA: Total Hip Arthroplasty 


\section{CHAPTER 1 \\ Thesis Overview}

The following thesis explores the topic of conservative hip implants, in particular biomechanical considerations pertaining to the surgical placement of such implants, and is divided into 8 distinct chapters.

This thesis begins with Chapter 2, which provides background information on the behaviour and composition of human bone, the total hip arthroplasty (THA) procedure, and examines the motivation behind conservative hip implants. The DePuy Silent Hip (DePuy, Leeds, UK) in particular is discussed as a novel straight short stem hip implant with minimal supporting biomechanical evidence, and no research to support one surgical implant position over another, which defines the scope of this thesis. Chapter 3 then expounds upon the objectives of this thesis, discussing the rationale behind the study, the research question and hypothesis, followed by the specific aims which this thesis hopes to achieve.

Chapter 4 presents an overview of the research in this field, reviewing literature pertaining to conservative hip implants currently on the market. A number of review papers are discussed initially, followed by papers evaluating conservative implants in a clinical setting, and those using biomechanical techniques. Finally, the findings are summarized and knowledge gaps are discussed.

Chapter 5 outlines the three phases of this thesis, and the methods used to accomplish each. Initially, relevant mechanical and material properties are discussed, including the material properties of synthetic bone and the chosen conservative hip implant. An overview of the biomechanical testing phase of this study is then provided, wherein 10 synthetic femurs are surgically implanted with the Silent Hip and axially loaded for the purpose of validating a corresponding finite element (FE) model. This is followed by a discussion of the finite element method (FEM) modelling technique, and the approaches used to generate the FE model are then discussed. Finally, design of experiments (DOE) is examined as a method for determining the optimal implant orientation given the full range of potential angles and positions. 
The results of these procedures are detailed in Chapter 6, which presents the outcomes for both the biomechanical testing phase and the FEM validation phase, followed by DOE results describing the optimal orientation of the Silent Hip within the proximal femur. Chapter 7 then discusses these findings and elaborates upon their clinical relevance and significance to the field, following which the limitations and potential future directions of this study are assessed. Finally, Chapter 8 comprises a summary section which gives an overview of the study and its subsequent findings. 


\section{CHAPTER 2 \\ Background}

\subsection{Human Femur Bones}

The human skeleton composes the framework of the body, and consists of a number of different bones whose functions vary. While some bones provide protection for internal organs, other bones are the main site for hematopoiesis, and still others aid in locomotion. The femur is a long bone within the leg that articulates with the hip and knee joints to allow for human mobility.

\subsubsection{Composition and Structure}

Bones, in particular long bones such as the human femur, must be both stiff and flexible in order to resist deformation while still enabling energy absorption at impact. ${ }^{1}$ Furthermore, bones must be light enough to allow human mobility. This dichotomy is overcome through the material properties and morphology of bone. Bones are composed of a bone matrix with varying proportions of inorganic mineral ions, water, and organic components. Type I collagen is the main organic protein in the bone matrix, while calcium phosphate (primarily hydroxyapatite) is the primary inorganic component. ${ }^{2}$ On a macroscopic level, bones are composed of cortical and cancellous (trabecular) bone. Cortical bone is the minimally porous, hard outer layer of bone, and is composed of lamellae that provide much of the required stiffness. Cancellous bone fills the bony interior and is composed of a woven porous network that makes the bone lighter, allows for blood vessel growth, and provides for some of the energy absorbing capabilities of bone. Bone mass is further reduced through the presence of a medullary cavity, in which the marrow is stored. ${ }^{1}$

\subsubsection{Biomechanical Properties}

When a load is applied to bone it resists deformation, and stress (measured as force/area) is developed in response. The local deformation that occurs due to this applied load is called strain, and refers to the relative change in size or shape. ${ }^{1}$ The reaction of bone to an applied load can be defined using either a load-deformation curve or a stress-strain curve. In a load-deformation curve, the amount of force needed to achieve a given deformation is defined, and thus this curve takes into account both the material properties of bone and its shape (Figure 1). Load and 
deformation are generally linearly related until the yield point, and this linear portion describes the elastic region of bone during which any deformations will not be permanent. After yield, the bone will begin to undergo permanent deformation until finally it fails. The slope of the elastic region describes the stiffness of the bone. ${ }^{1}$

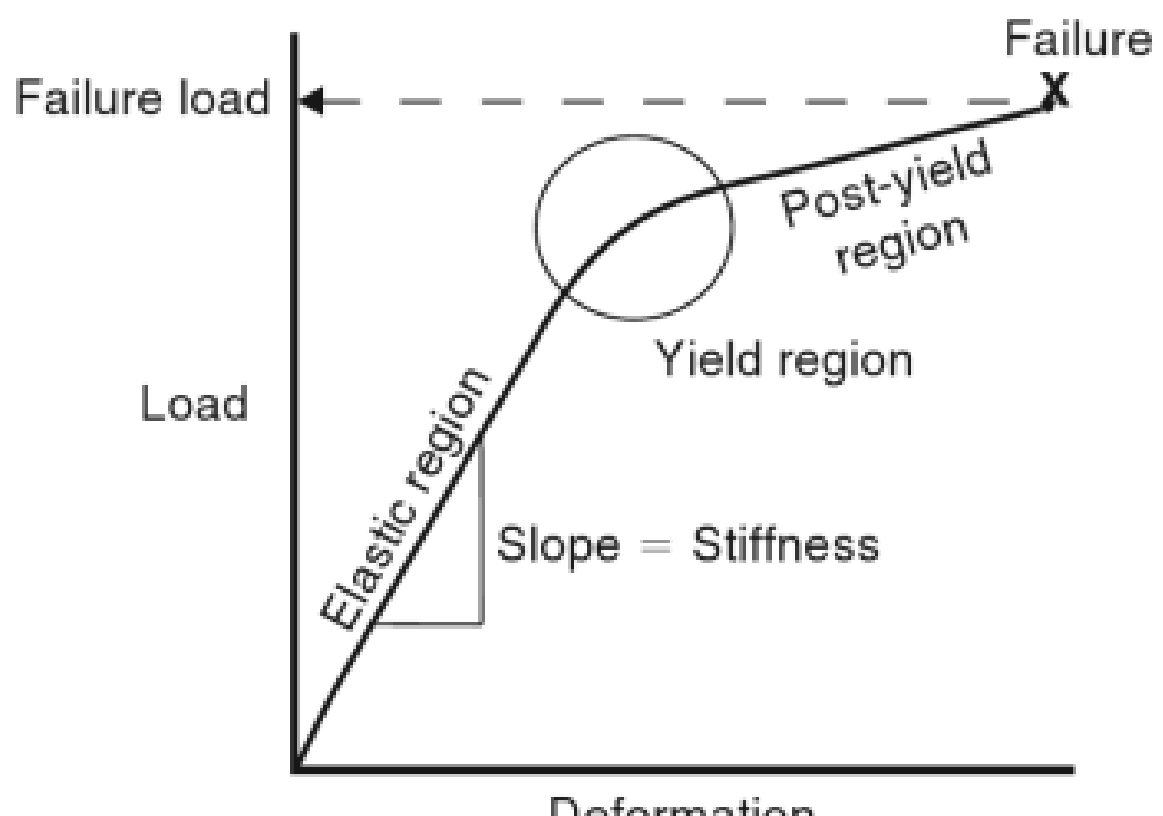

\section{Deformation}

Figure 1: Load versus deformation plot for bone, indicating the elastic and yield regions ${ }^{1}$

The stress-strain curve for bone reflects the material behaviour of bone, and contains: an elastic region whose slope is known as the Young's modulus (or elastic modulus) which measures bone rigidity, a yield point with a corresponding yield stress and strain, and an ultimate point following which bone enters the inelastic region and subsequently fails. ${ }^{1}$ Bone is a viscoelastic material, and so its strength is influenced by the rate of strain. For example, a higher strain rate increases the apparent stiffness of bone and so causes it to fracture at higher forces, where a strain rate of $0.01 \mathrm{~s}^{-1}$ to $0.03 \mathrm{~s}^{-1}$ represents physiological strain rates during rapid walking and running respectively (Figure 2). In general, bone typically fails at a deformation of about $1 \% .^{1-4}$ 


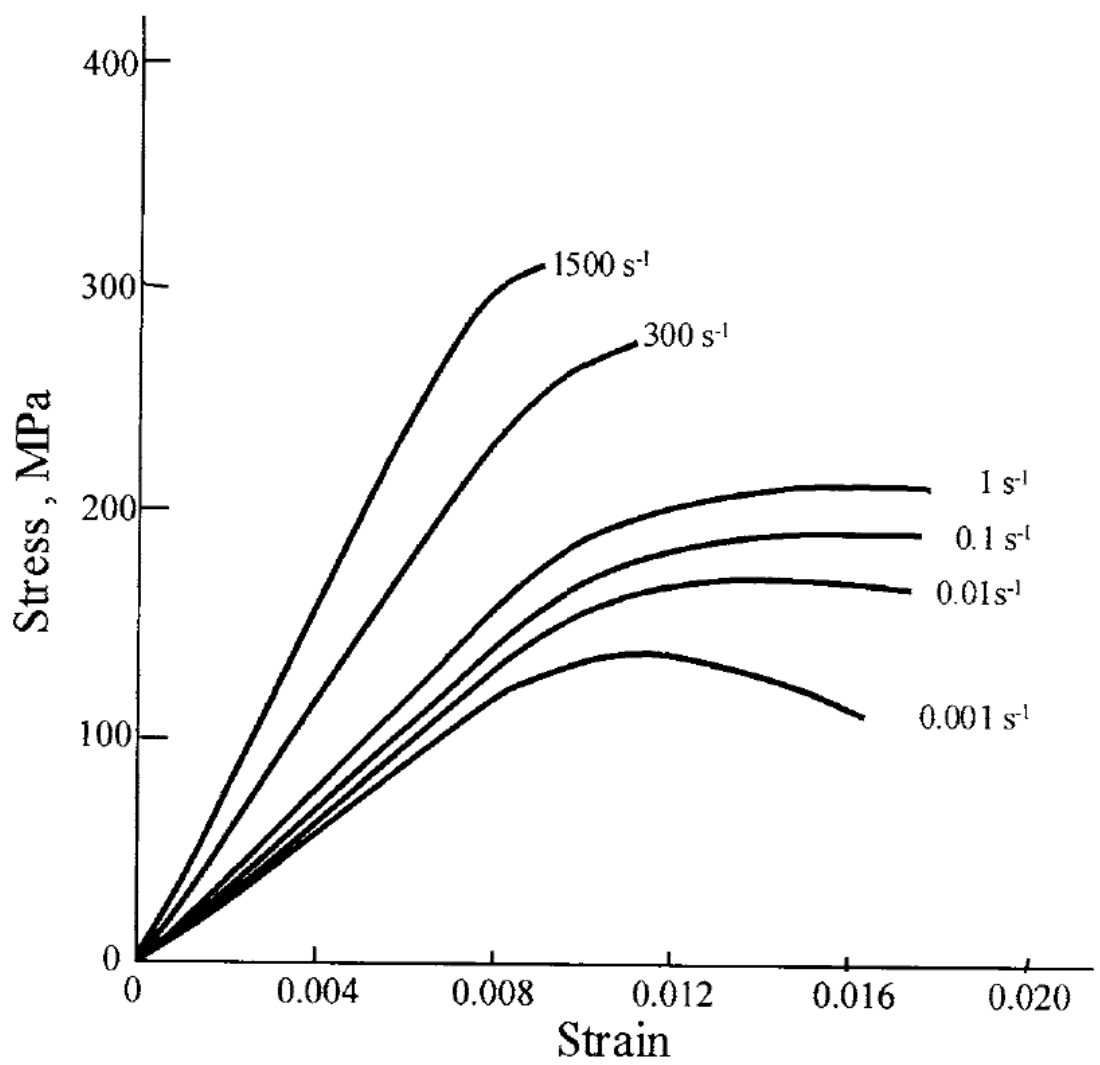

Figure 2: Stress-strain curve for bone, demonstrating the influence of strain rate on behaviour ${ }^{2,5}$

Due to the presence of directional constituents, such as lamellae, haversian canals and collagen, bone is anisotropic. This means that it responds differently to mechanical stimuli depending on the direction of load application. ${ }^{1}$ In the femur, anisotropy makes the bone strongest in its primary loading direction, namely axial compression (Table 1). Density also plays a role in bone strength, with increasing density generally being associated with higher compressive strengths. ${ }^{2}$ Bone is also an inhomogeneous material, both at the macroscopic and microscopic levels. As such, the elastic modulus for bone varies depending on both the large-scale anatomic location and the particular microscopic bone lamella. ${ }^{6}$

Table 1: Average mechanical and material properties for human femoral bone ${ }^{2,7}$

\begin{tabular}{|c|l|ccc|}
\hline \multicolumn{1}{c|}{ Bone } & \multicolumn{1}{|c|}{ Loading Mode } & $\begin{array}{c}\text { Density } \\
\left(\mathbf{g} / \mathbf{c m}^{\mathbf{3}} \mathbf{)}\right.\end{array}$ & $\begin{array}{c}\text { Ultimate Strength } \\
(\mathbf{M P a})\end{array}$ & $\begin{array}{c}\text { Young's Modulus } \\
\text { (GPa) }\end{array}$ \\
\hline \multirow{4}{*}{ Cortical } & Longitudinal Tension & $1.6-2.1$ & 133 & 17 \\
\cline { 2 - 5 } & Longitudinal Compression & $1.6-2.1$ & 195 & 17 \\
\cline { 2 - 5 } & Longitudinal Shear & $1.6-2.1$ & 68 & 3.3 \\
\cline { 2 - 5 } & Transverse Tension & $1.6-2.1$ & 51 & 11.5 \\
\cline { 2 - 5 } & Transverse Compression & $1.6-2.1$ & 33 & 11.5 \\
\hline Cancellous & Compression & $0.1-1.0$ & 6 & 0.76 \\
\hline
\end{tabular}




\subsection{Total Hip Arthroplasty}

Attempts at hip replacement have been recorded as early as 1891 in Germany, in which ivory was used to replace the femoral heads of patients whose joints had degenerated due to tuberculosis. $^{8}$ The total hip arthroplasty (THA) procedure as it is known today was first established by Sir John Charnley in the early 1960's, and is one of the most successful orthopaedic procedures of the $20^{\text {th }}$ century. ${ }^{8}$ Presently, THA is an elective surgical procedure in which the surgeon removes damaged sections of the hip joint and replaces them with prosthetics made from metal alloys, ceramic, or plastic.

\subsubsection{Indications for Total Hip Arthroplasty}

In a healthy hip joint, the femoral head articulates smoothly with the acetabulum due to the lubricating effect of the synovial fluid and the smooth articular cartilage, which also acts to cushion the hip joint during load bearing. However, with age and repeated overuse, this cartilage may begin to degenerate, causing the bones of the femoral head and acetabulum to grate against one another. This can lead to the formation of bony spurs called osteophytes, swelling in the joint, and ultimately pain and reduced flexibility (Figure 3). ${ }^{9,} 10$ This type of degenerative joint disease is called osteoarthritis (OA), and is the most common reason for joint replacement surgery in Canada, accounting for $82.1 \%$ of primary hip replacements. ${ }^{11}$

Other indications for THA include acute hip fractures, rheumatoid arthritis, osteonecrosis, and childhood hip diseases; however, all of these diagnoses combined are responsible for only $13.9 \%$ of hip replacements in Canada. ${ }^{11}$ 


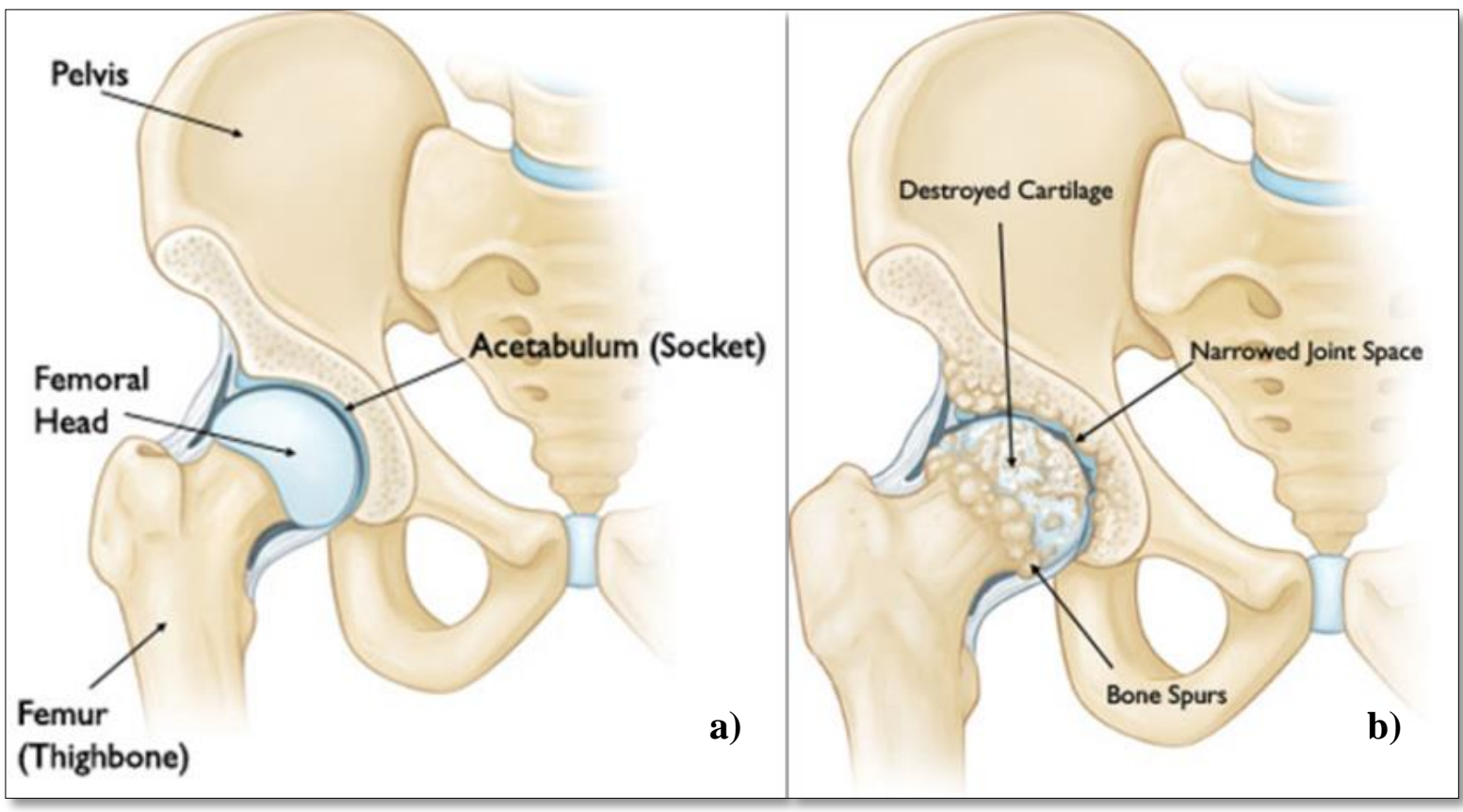

Figure 3: a) Anatomy of a healthy hip joint; b) Outcomes of an osteoarthritic hip joint ${ }^{12}$

\subsubsection{Hip Replacement Demographics}

Presently, orthopaedic surgeons make recommendations for hip replacement surgery based on the patient's pain and mobility levels, in addition to their overall health. ${ }^{12}$ While certain health conditions may contraindicate hip replacement surgery, an operable patient who is both healthy and active will be more likely to have a successful outcome. ${ }^{13}$ Thus while THA used to be reserved for those aged 60 and over, it is increasingly being successfully performed in the younger patient population in order to maintain quality of life. ${ }^{13,14}$ Furthermore, the prevalence of arthritis in the younger population is predicted to increase by half in the coming years, suggesting that THA in this population will become even more widespread. ${ }^{15}$

However, most hip implants have a functional lifespan of only $10-20$ years before a revision surgery is required, due to factors that include aseptic loosening, osteolysis and decreased implant function. ${ }^{16}$ Thus, undergoing hip replacement surgery at a younger age introduces the possibility that a revision surgery will be required within the patient's lifetime. ${ }^{17}$ 


\subsubsection{Revision Surgery}

Revision surgery is a complex procedure that requires removal of the failed component, reconstruction of the remaining bone and soft-tissue, and then determination of enough good bone stock to ensure fixation of the new implant. ${ }^{16}$ Secure fixation of the revision component is crucial to ensuring long-term survivorship of the implant. ${ }^{18}$ Traditional hip implants extend into the diaphysis of the femur and are cemented in place or undergo osseointegration along their length, making them difficult to remove. ${ }^{16}$ This often makes stable fixation challenging to achieve proximally, as this bone stock tends to be deficient and weak from the previous implant. $^{18}$

Manufacturers have therefore begun designing conservative hip implants that preserve a greater portion of healthy proximal bone stock initially to facilitate future revision surgeries if necessary. These hip implants include short stem or ultra-short stem designs that are usually uncemented and designed to be surgically implanted within the metaphysis of the proximal femur. ${ }^{19,20}$

\subsubsection{Cemented and Cementless Implants}

Hip implants can be affixed within the femur through either cemented or cementless means. In cemented THA, an acrylic polymer called polymethylmethacrylate (PMMA) is used to mechanically interact with the implant and the bone, providing stable fixation for the implant. Cementless THA utilizes a textured implant surface topography, often with a hydroxyapatite (HA) coating, that encourages direct bony ongrowth and ingrowth. There is much debate as to the efficacy of one fixation method over another, with the main difference in survivorship arising from the age of the patients at the time of surgery. Revision rates for cemented stems were found to be higher in the younger patient population when compared to the older population, typically due to aseptic loosening and osteolysis. ${ }^{21-23}$ The 10-year survivorship of cementless THA was found to be significantly better than cemented THA, particularly in the younger population. ${ }^{21,24}$ Use of cementless fixation has been steadily increasing in North America, and in Canada, cementless THA is currently the most common fixation method. ${ }^{11}$ 


\subsubsection{Stress Shielding}

Hip implants have begun to diverge from designs based on the Koch model of the hip. ${ }^{25}$ According to this model, the axial forces experienced during normal walking act as compressive and tensile loads on the medial and lateral proximal femur respectively. ${ }^{26}$ It is now known that the iliotibial band, which runs concurrent to the lateral edge of the femur, acts as a tension band to turn lateral tensile forces into a compressive load. ${ }^{26}$ This is important when considering implant design, as a good implant should therefore have the capacity to laterally distribute load in the proximal femur in order to align with physiological conditions. ${ }^{25}$

Physiological loading is defined as load transfer that produces a stress distribution equivalent to that present in a healthy hip joint under normal loading conditions. In THA, achievement of physiological loading imparts biomechanical stability to the implanted femur, and so is thought to prevent a cascade of events initiated by "stress shielding," in which the host bone is understressed due to the stiff femoral component supporting the majority of the load. According to Wolff's law, bone is formed or resorbed in accordance with the magnitude and direction of the applied forces. ${ }^{27}$ Inclusion of a femoral hip component would therefore cause the loads felt by the bone to decrease, eliciting bone adaptations that include decreased mass and increased porosity (Figure 4). ${ }^{28}$ This can lead to the development of osteopenia, bone resorption around the implant, loosening of the implant with a concurrent decrease in biomechanical stability, and ultimately failure, which can necessitate a revision surgery in compromised bone stock. ${ }^{29-31}$

Stress shielding can be difficult to define clinically, as a bone mineral density (BMD) loss of approximately $30 \%$ is required before changes can be seen radiographically. ${ }^{32}$ For that reason, a qualitative four degree system of bone remodelling is typically utilized when evaluating the severity of stress shielding. ${ }^{33}$ In this system, degrees 1 and 2 represent low levels of stress shielding represented by decreased cortical bone density in only the proximal femur. More severe stress shielding is defined by degrees 3 and 4, which characterize bone resorption extending into the diaphysis. ${ }^{34}$ 


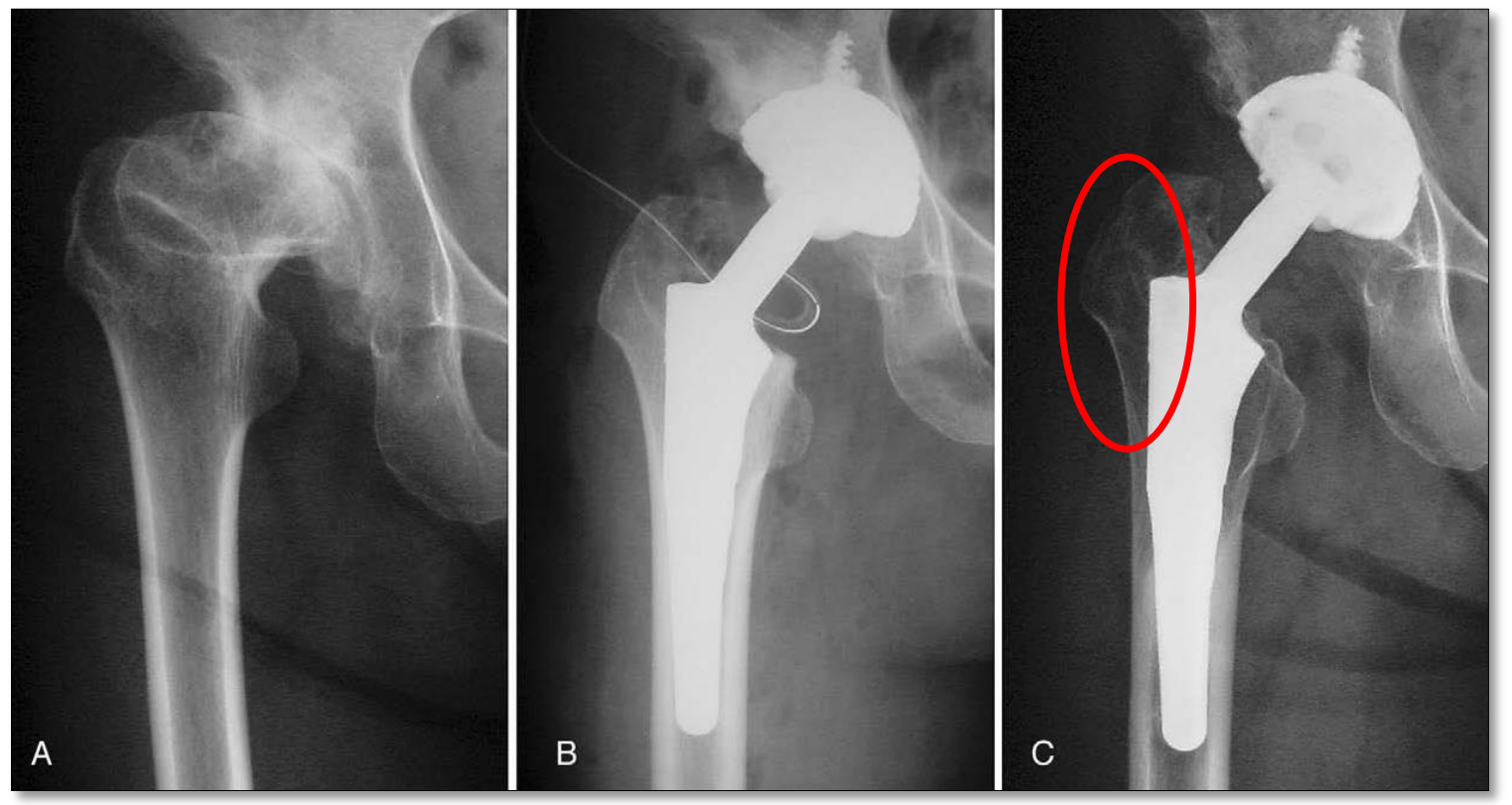

Figure 4: Radiographs of the right hip: a) before implantation; b) immediately postoperatively; c) 7.5 years after surgery, with severe stress shielding noted ${ }^{35}$

Many implants have been designed with a lateral flare in order to engage the metaphysis and maintain proximal loading. ${ }^{29}$ Furthermore, studies have found that short stem hip implants, which do not extend as far into the femoral diaphysis as traditional hip implants, exhibit more physiological loading proximally with less BMD loss. ${ }^{31,36,37}$

\subsubsection{Gruen Zones}

In 1979, Gruen et al. developed a system to standardize radiographic assessment of loosening around the femoral component interface by dividing it into 7 zones (Figure 5). ${ }^{38}$ This method was initially used to determine the location and type of loosening present in cemented femoral hip components. ${ }^{38}$ Currently, these zones are used as a standardized method to compare spatial and temporal BMD measurements across varying implants types. They are therefore useful when evaluating stress shielding, as it is possible to explore the progression of BMD loss or gain within consistent areas of the proximal femur. 


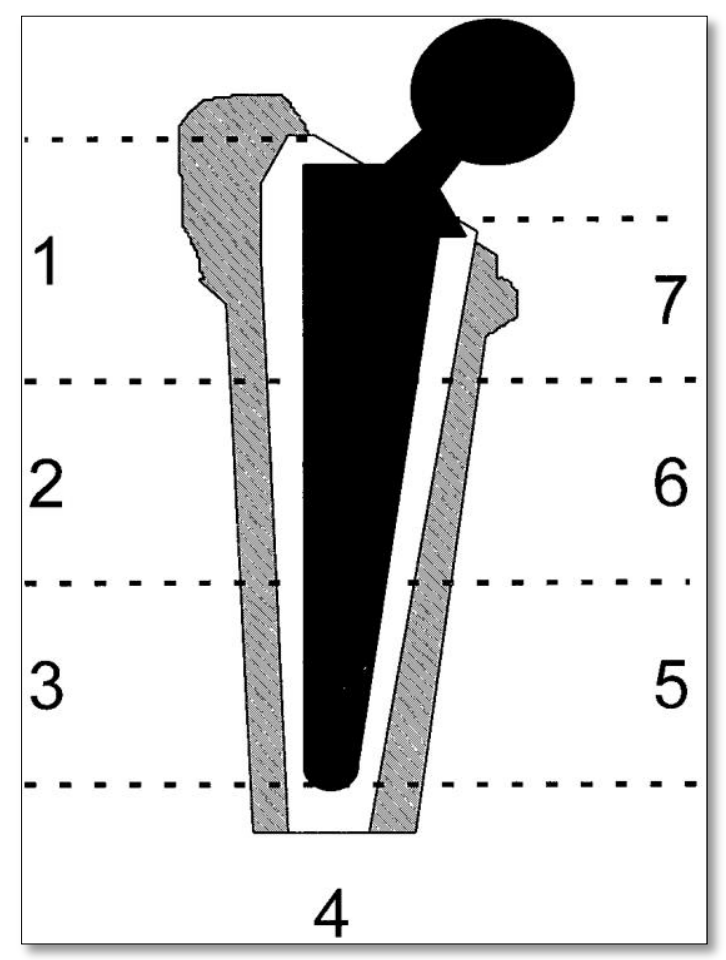

Figure 5: Diagram depicting the seven Gruen Zones ${ }^{39}$

\subsection{Conservative Hip Implants}

Short stem hip implants can vary significantly in geometry; however, they are all similar in that they extend only minimally into the femoral diaphysis, and are intended for metaphyseal fixation (Figure 6). ${ }^{36,}$ 40-44 These stems are typically designed for a minimally invasive surgical implantation technique, and a high femoral neck cut to allow for optimal bone stock preservation. $^{25,45}$ Maintaining the neck also ensures that the trabecular system of the metaphyseal cancellous bone remains intact, thereby allowing for more physiological stress propagation through the femur. ${ }^{46}$ Mid-term results for conservative implants have been favourable, illustrating that these implants have good initial stability, a tendency to provide more physiological conditions with less bone resorption, and a good implant survival rate. ${ }^{25,36,37,41,46-}$ ${ }^{48}$ However, there is a dearth of data supporting short stem implants, and hence there is still a need for more biomechanical and clinical analyses. ${ }^{44}$ 


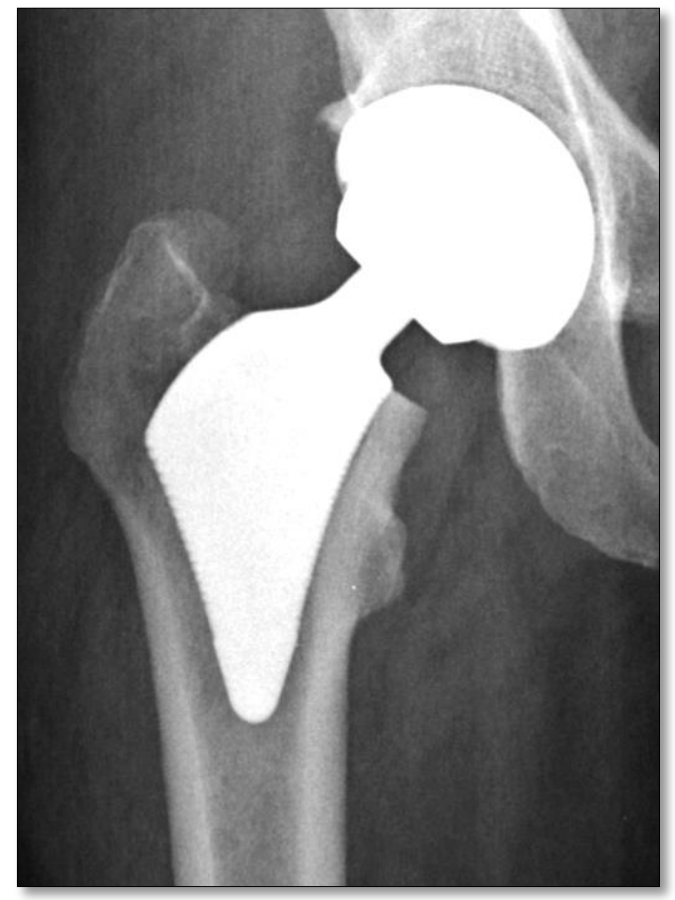

Figure 6: A short stem hip implant with a lateral flare for metaphyseal fixation ${ }^{20}$

\subsubsection{DePuy Silent ${ }^{\mathrm{TM}}$ Hip Implant}

The DePuy Silent ${ }^{\mathrm{TM}}$ Hip (DePuy, Leeds, UK) is an example of a modular, cementless short stem hip implant with limited supporting clinical or biomechanical data. It features a straight short stem with a collarless tapered profile for implantation in the femoral neck (Figure 7). It is composed of a Ti 6Al-4V alloy, and utilizes the DuoFix ${ }^{\mathrm{TM}}$ fixation system, which consists of a layer of hydroxyapatite coated beads to promote bony ingrowth. A 12/14 taper enables the Silent Hip to be combined with either a ceramic or metal head, for optimal choice of friction couple. ${ }^{17}$ 


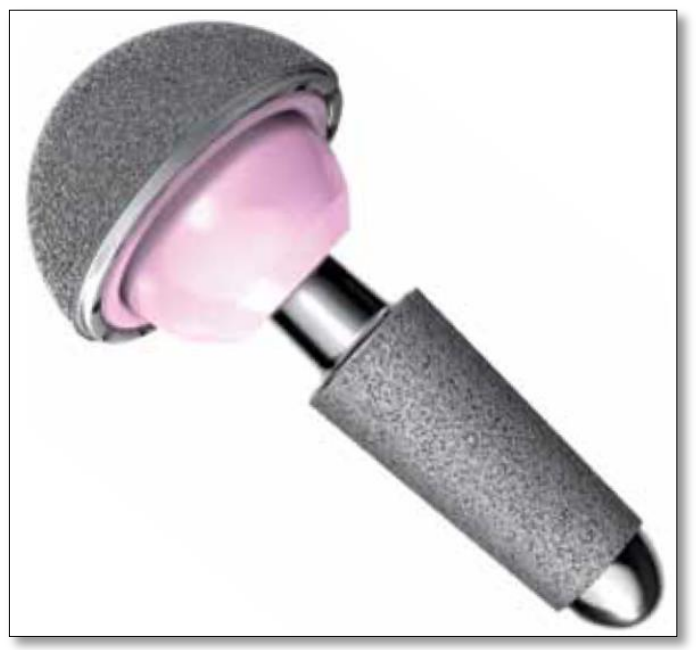

\section{Figure 7: Illustration of the DePuy Silent Hip (DePuy, Leeds, UK) ${ }^{17}$}

This implant was designed specifically for younger, active patients with primary or secondary arthritis, for which hip resurfacing may not be suitable. ${ }^{49}$ It facilitates minimally invasive surgical implantation techniques, enabling preservation of soft tissues and the potential for earlier recovery and rehabilitation. A very high neck cut preserves maximal bone stock, and the tapered stem engages the proximal femur to distribute loads in a physiological manner (Figure 8). ${ }^{17}$ Contraindications for this hip implant include poor bone quality or gross deformity in the femoral neck, morbid obesity, and males over the age of 65 years or females over the age of 60 years. $^{49}$

A pilot clinical study used radiostereometric analysis (RSA) to evaluate implant stability, finding acceptable levels of femoral stem migration at two years ( $<1 \mathrm{~mm}$ in any direction), and no evidence of continuing migration at five years to suggest implant loosening. ${ }^{50}$ The Kaplan-Meier survivorship at three years based on stem revision was 97\% (95\% CI of 94-100\%). ${ }^{51}$ While these are promising results, further long-term clinical evidence and biomechanical research would still be beneficial in fully understanding how this implant functions within the femur. 


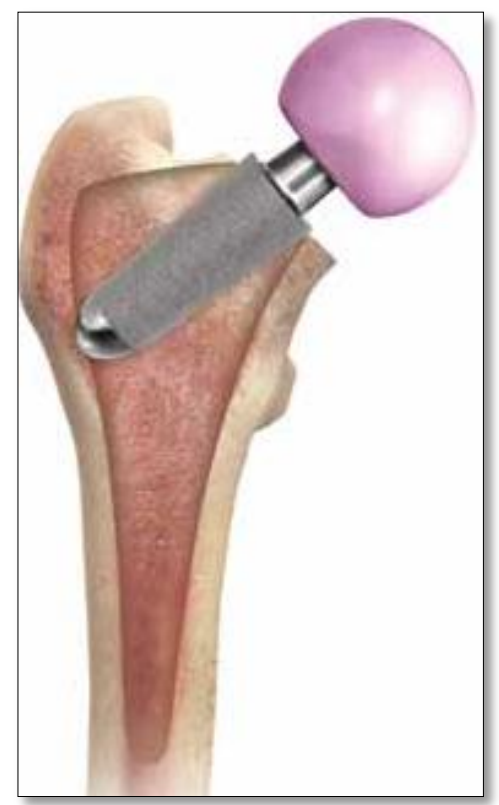

Figure 8: Silent Hip implanted in the femoral neck, demonstrating high neck cut ${ }^{17}$

\subsubsection{Examination of Orientation}

One area of research on conservative hip implants for which there is minimal data is the optimal orientations of these stems within the femur. Biomechanical studies examining the varus/valgus placement of the femoral component of hip resurfacing arthroplasties have shown a valgus orientation to be optimal. It was found that a valgus placement increased the proximal femoral strength of the femur, and so resulted in a higher femoral fracture load. ${ }^{52,53}$ Valgus placement was also found to induce strain patterns more similar to an intact femur, suggesting physiological loading. ${ }^{54}$ These results were further supported in a study that found valgus to be the optimal coronal alignment of the Birmingham Mid-Head Resection (BMHR) (Smith \& Nephew, Marl, Germany) to increase proximal femur strength and provide protection in the case of superior femoral notching. ${ }^{55}$

Another study assessed the effect of femoral head lag screw positioning on proximal femur stiffness and load-to-failure. It was determined that a central position in the lateral plane coupled with an inferior orientation in the anteroposterior plane significantly maximized both axial stiffness and load-to-failure. ${ }^{56}$ However, no prior studies have examined the ideal 3D orientation of conservative short stem hip implants to optimize biomechanical stability. 


\section{CHAPTER 3 \\ Thesis Objectives and Hypothesis}

\subsection{Research Objective and Rationale}

There are numerous conservative hip implants on the market that optimize lateral flare and are designed for femoral metaphyseal fixation; however, there is no corresponding biomechanical research on the ideal 3D orientation of these devices in order to enhance long-term mechanical stability of the femur-implant interface and extend the implant's service life. The goal of this study was therefore to determine the optimal 3D angle and position of a straight, short stem hip implant designed for metaphyseal fixation to achieve maximal biomechanical stability.

\subsection{Research Question}

What is the 3D orientation of a straight short stem hip implant within the proximal femur that will yield a stress distribution most similar to that of an intact femur, thereby providing optimal physiological loading?

\subsection{Research Hypothesis}

Given that the stem of a straight, short stem total hip replacement, such as the Silent Hip, has a similar geometric structure to hip resurfacing implants, the BMHR, and lag screws, it is postulated that the Silent Hip will be biomechanically similar. It was therefore hypothesized that a valgus and inferior coronal plane orientation of the stem combined with a neutral lateral placement would result in an optimal stress distribution and superior biomechanical stability.

\subsection{Specific Aims}

This research endeavoured to assess the optimal orientation of a straight, short stem hip implant within the proximal femur through:

A) Biomechanical Testing

a. Biomechanical testing was completed on implanted femurs that corresponded to six maximum achievable angles and positions of the hip implant, in addition to neutral. 
B) Finite Element Analysis (FEA)

a. Biomechanical tests were used to validate a corresponding finite element (FE) model of the implant-femur construct.

b. The validated model was then parameterized such that the range of implant angles and positions could be attained.

C) Design of Experiments (DOE)

a. DOE was used to optimize the parameterized FE model by determining the orientation which yielded the minimum difference from the intact femur in average proximal cortical bone stress.

This study will have clinical implications for the optimal surgical placement of straight, short stem hip implants within the proximal femur. It will also be one of the few existing biomechanical studies on any type of short stem hip implant, and the first study to optimize the 3D orientation for superior biomechanical stability. 


\section{CHAPTER 4 \\ Relevant Literature}

\subsection{Conservative Hip Implant Reviews}

There are a few studies which have examined available conservative hip implants, with the purpose of appraising their biomechanical characteristics and short- to mid-term clinical results. In their review, Valverde-Mordt et al. ${ }^{20}$ determined that currently available prostheses ranged from shortened classic stems (e.g. short modular femoral stem) to metaphyseal wedged stems (e.g. Mayo) (Figure 9). A newer line of implants included ultra-short stem devices with a high neck cut (e.g. Proxima) and prostheses which are designed to be used when a hip resurfacing procedure has failed or is not indicated (e.g. BMHR) (Figure 10). Shortened classic stems were determined to have too few available published results to conclusively establish their primary stability, while metaphyseal wedged stems were found to have a good survival rate with less bone resorption than traditional long-stem prostheses. However, hip implants such as the Mayo stem (Zimmer, Rochester, MN, USA) had reports of perforation of the external cortical bone, intraoperative fractures, and post-operative complications. 


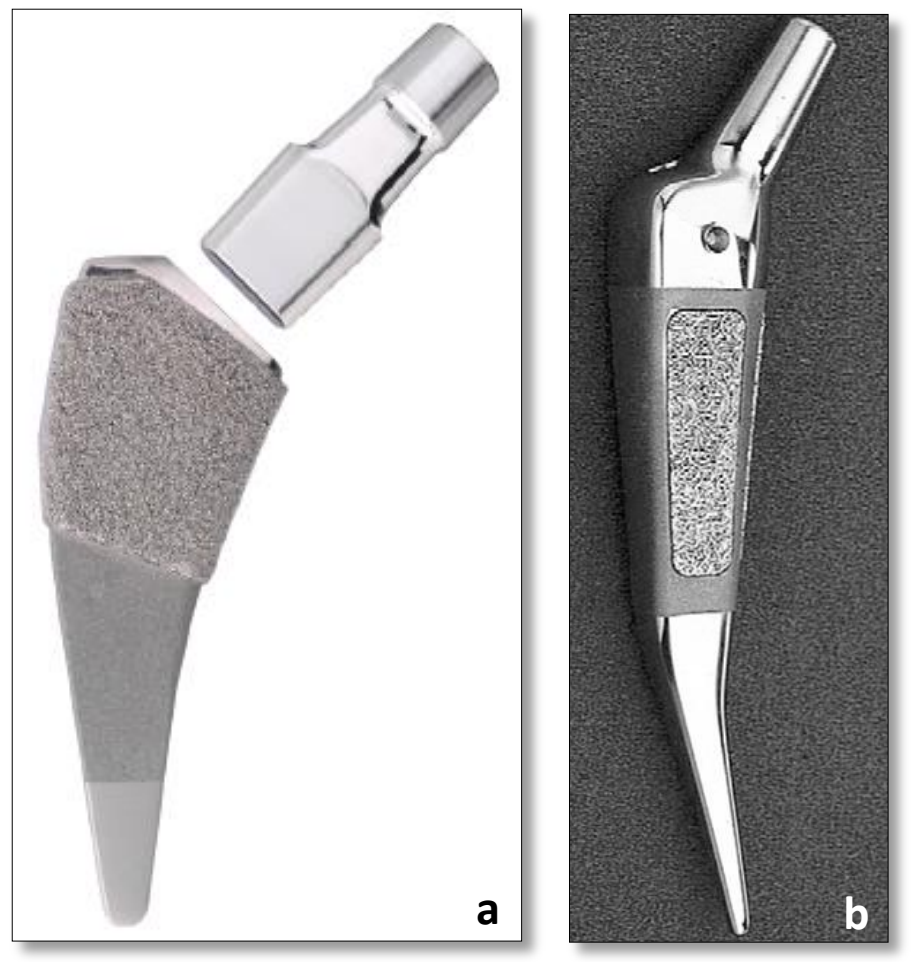

Figure 9: a) Short modular femoral stem (Smith \& Nephew, Inc., Memphis, TN, USA) ${ }^{57}$; b) Mayo conservative implant double wedge stem with proximal coating (Zimmer, Rochester, MN, USA) ${ }^{20}$

The Proxima (DePuy, Leeds, UK) was found to have early primary stability, greater bone sparing effects, and decreased fracture risk in young patients with good bone quality, as its lateral flare and short stem allowed a conservative high neck cut with metaphyseal fixation. The Birmingham mid-head resection (BMHR) (Smith \& Nephew, Marl, Germany) was defined as an intermediate prosthesis designed for osteonecrotic patients and as a secondary implant when a hip resurfacing fails. It consists of a solid metal hemisphere with a porous-coated stem for fixation in the femoral neck, and a metal-on-metal couple with the acetabular component. 

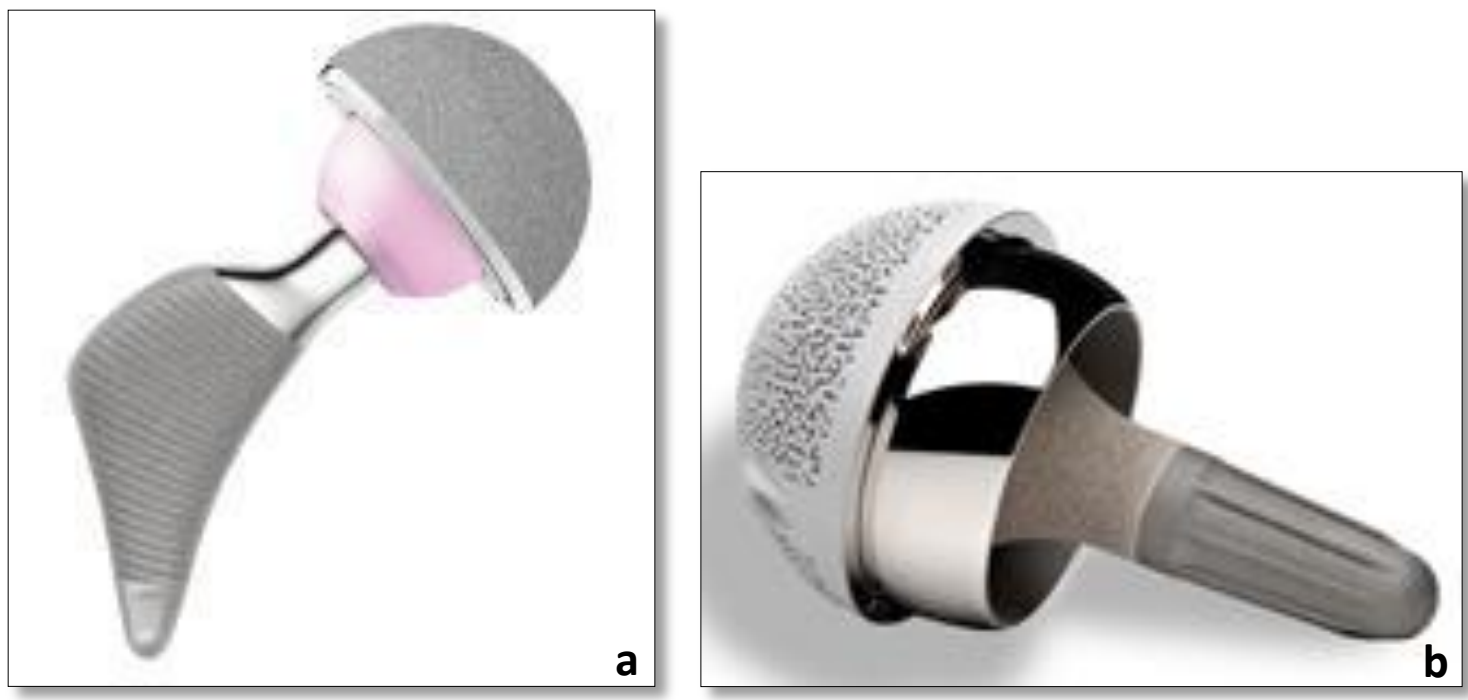

Figure 10: a) Proxima Hip (DePuy, Leeds, UK) ${ }^{57}$; b) Birmingham mid-head resection (BMHR) (Smith \& Nephew, Marl, Germany) ${ }^{58}$

A review completed by Kluge ${ }^{19}$ found that implants which have a stem extending into the femoral diaphysis exhibited stress shielding and bone density loss, while implants with proximal load transfer and no distal stem fixation required less intraoperative bone removal, preserved proximal bone stock in the long term, and had better physiological loading. However, it was noted that hip implants relying solely on metaphyseal fixation required sufficiently good cancellous bone quality to ensure primary stability.

Both Kluge and Valverde-Mordt et al. briefly mentioned the Silent Hip, which is a new hip implant similar in design to the BMHR (Figure 11). According to Kluge, this tapered press-fit implant, designed for fixation in the femoral neck and proximal metaphysis, allowed for nearly physiological loading. ${ }^{19}$ Valverde-Mordt et al. stated that the Silent Hip is still in the clinical trial phase and therefore no conclusive evidence can be drawn; however, it could prove to be a very versatile implant given its 12/14 taper that allows the surgeon a choice of acetabulum and friction couple. ${ }^{20}$ 


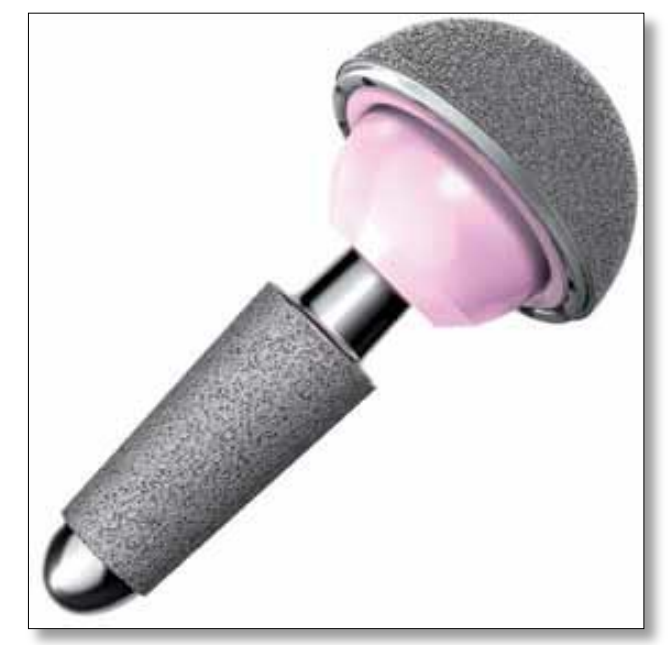

Figure 11: Silent Hip (DePuy, Leeds, UK) ${ }^{17}$

\subsection{Retrospective Reviews}

Oldenrijk et al. ${ }^{44}$ completed a systematic review of the revision rates reported in 49 clinical short stem hip arthroplasty studies. There were 19 different types of stems used overall, which they classified into three stem categories: "collum" (straight neck-sparing stems), "partial collum" (partial femoral neck-sparing), and "trochanter-sparing" (shortened tapered stem but not necksparing). Of these stem types, only 3 fell under the collum classification. As such, the clinical evidence for these stems was limited to a small number of studies with mid-term results, evaluating primarily the CUT (ESKA Implants GmbH \& Co, Luebeck, Germany) stem. Overall, it was found that partial collum and trochanter-sparing stems displayed adequate mid-term survival rates (above $90 \%$ survival at 10 years of follow-up), while collum stems did not.

When looking at some of the individual conservative stem reviews available, the results are slightly more favourable. Choy et al. ${ }^{59}$ completed a retrospective review in which data from the Australian Orthopaedic Association National Joint Replacement Registry was used to compare the survivorship of short Exeter stems with the standard-length Exeter implants (Stryker, Kalamazoo, Michigan, USA). It was found that there was no significant difference in the revision rates between standard and short stems, and short stems had the advantage of being able to fit smaller patient sizes, for whom oversizing and subsequent component loosening and failure is a common concern. 
Xing et al. ${ }^{60}$ looked at the effect of stem length on reoperation and complication rates for patients with proximal femur metastases. They retrospectively reviewed 203 patients with proximal femur metastases who had been treated with hip implants ranging in size from short stem (12 $14 \mathrm{~cm}$ stem length) to long stem $(25-35 \mathrm{~cm})$. It was discovered that short stem implants had a slightly higher reoperation rate than long stems (8.6\% as compared to $1.4 \%)$ due to aseptic loosening and periprosthetic fracture, which was attributable to poor proximal bone quality. However, long stems were associated with a much higher risk of cardiovascular complications and a correspondingly increased postoperative complication rate. This implies that long stems might not always be indicated depending on the quality of the proximal bone stock.

\subsection{Clinical Studies}

There are a number of clinical studies that have evaluated the performance of individual conservative hip implants, including custom-made implants. Santori et al. ${ }^{43}$ published mid-term results for a clinical trial in which 129 custom-made short stem hip implants were inserted into 109 patients. There were two types of hip replacements initially implanted: Type A consisted of implants with a short stem extending no more than $3 \mathrm{~cm}$ past the lesser trochanter, while Type B were ultra-short stem implants with a lateral flare shape (Figure 12). The patient criterion for the clinical trial included an age of less than 60 years and good bone stock, and the follow-up period was 11.8 years for Type A implants and 6.9 years for Type B. Radiographs of the hip replacement site were taken immediately post-operatively, and at 3 months, 6 months, 1 year and then annually. 


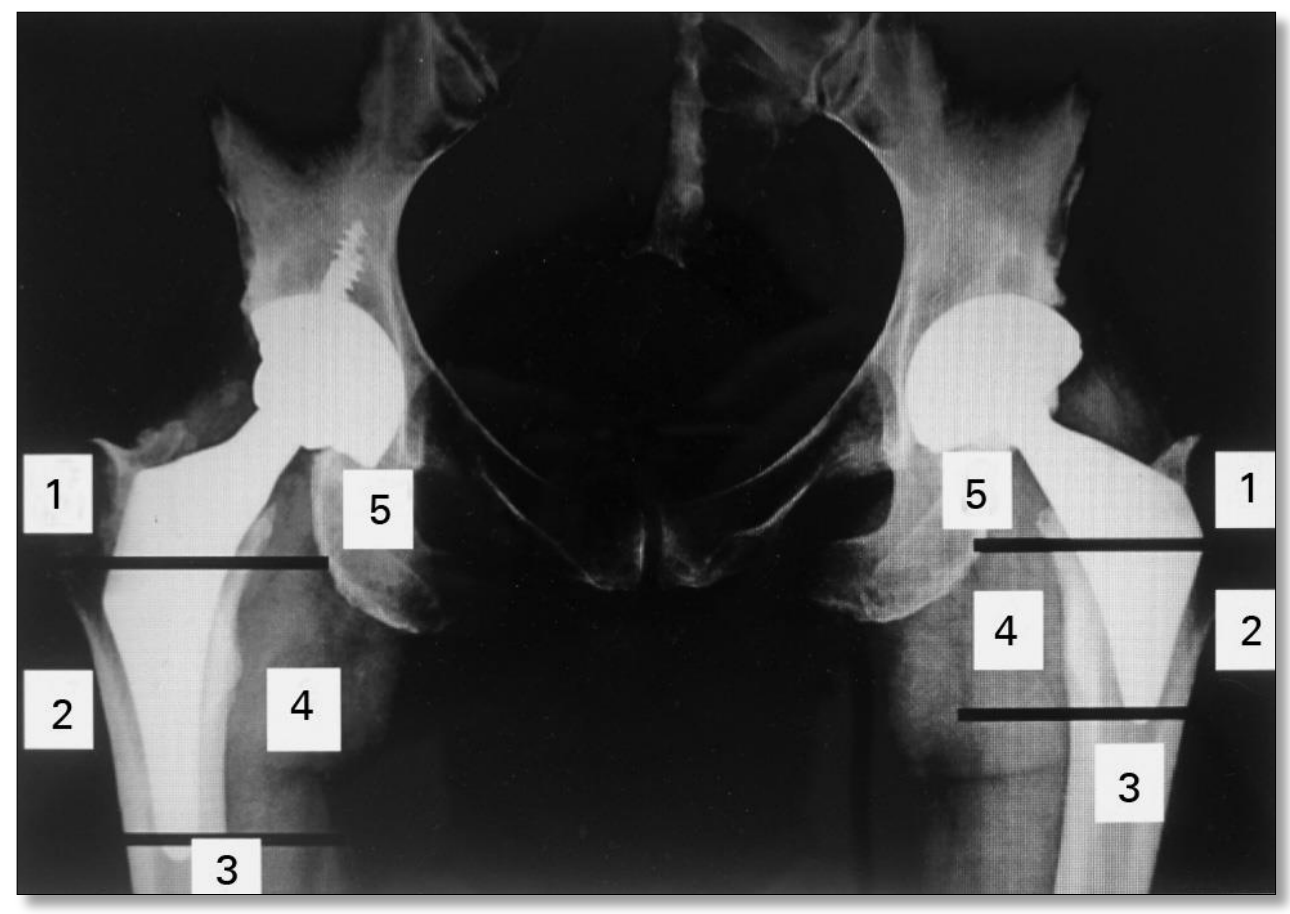

Figure 12: Radiograph of an implanted Type A short stem hip implant (right hip) and a Type B ultra-short stem implant (left hip), with a numerical depiction of the 5 modified Gruen zones for short stem implants 43

Santori et al. ${ }^{43}$ then consolidated patient data and measured the mid-term implant alignment, endosteal spot welds, and proximal bone resorption among other features. ${ }^{43}$ Stress-shielding only occurred in Type A implants, which also exhibited a decreased bone mineral density in all modified Gruen zones. Conversely, Type B implants showed spot welds in Gruen zone 2 and an increased bone density in zones 2 and 4, indicating that stress was distributed to the lateral proximal femur. These conclusions are supported in a previous study by Santori et al., ${ }^{25}$ which determined that the bone mineral density of ultra-short stem implants 2 years post-operatively was nearly identical to the non-operated contralateral hip. This implies that for young patients with good metaphyseal spongy bone, it is possible to rely on this trabecular bone for complete lateral load transfer.

In a prospective cohort study of the collum femoris preserving (CFP) prosthesis (Waldemar LINK GmbH \& Co, Hamburg, Germany), Hutt et al. ${ }^{48}$ assessed the Harris Hip Scores (HHS), radiographic lucency, and implant stability of 67 implanted stems at 10 years. They found a statistically significant improvement in HHS from those reported preoperatively (mean of 50 preoperatively and 91 postoperatively), and no radiographic evidence of loosening or osteolysis. Overall, the stems were found to be stable with good bony ingrowth. 
Similar measures were evaluated in a clinical study by Carlsson et al., ${ }^{41}$ in which the Gothenburg osseointegrated titanium (GOT) (Figure 13) hip arthroplasty was implanted in 20 patients, following which the HHS, implant migration, and radiographic lucencies at 2 years postoperatively were determined. HHS were high (>90) at 2 years, and radiographic analysis showed only minor resorption under the collar of the femoral component. Radiostereometric analysis (RSA) was used to evaluate implant migration and rotation, and only minimal movement was noted (within the threshold of $1.2 \mathrm{~mm}$ ). These results indicate that the implant has achieved osseointegration in the metaphysis, with good predictions for long-term stability.

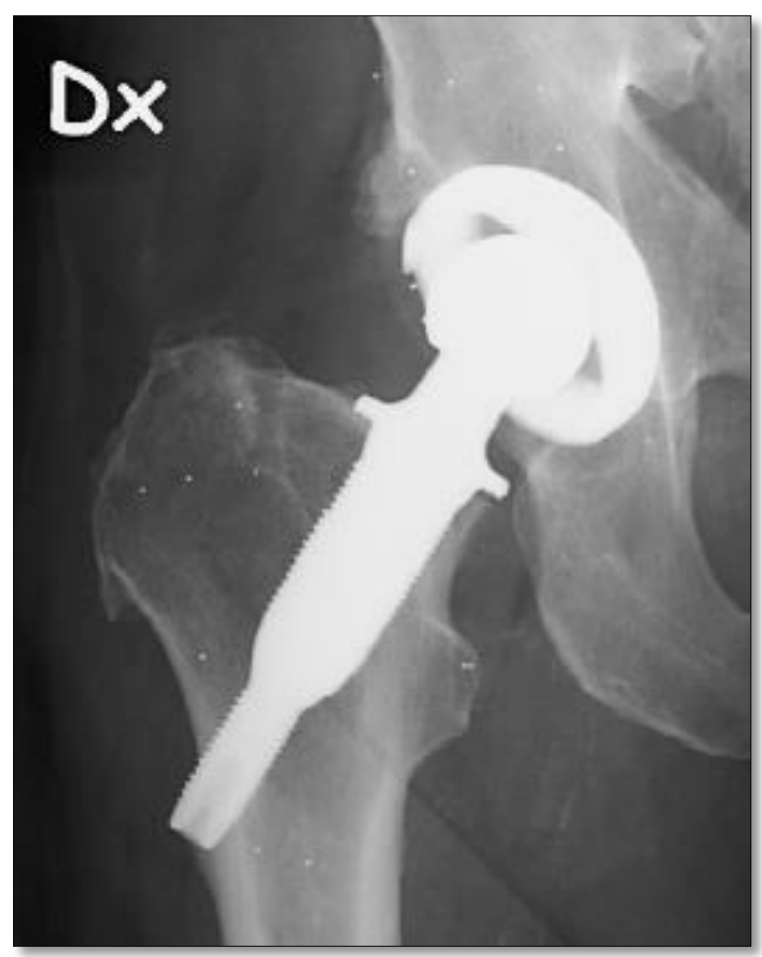

Figure 13: Radiograph of an implanted Gothenburg osseointegrated titanium (GOT) arthroplasty 41

Finally, Tóth et al. ${ }^{40}$ presented a study examining the short-term outcomes of the Proxima ${ }^{\mathrm{TM}}$ (DePuy, Leeds, UK) short stem hip implant. There were 35 patients included in the clinical trial with a total of 41 implanted hips, and HHS were recorded pre- and postoperatively. Mean 2 year HHS were 88 and radiographic analysis showed no signs of loosening or migration. However, it was noted that 2 stems were implanted in severe varus and 8 in varus orientation, as a result of the implant shape and "round the corner" broaching method. 


\subsection{Biomechanical Studies}

\subsubsection{Finite Element Modelling Studies}

While many suggest that conservative metaphyseal implants are the optimal solution for bone conservation and proximal load transfer, not all investigations are in agreement. In a study by Van Rietbergen et al., ${ }^{61}$ finite element analysis (FEA) was used to determine whether shortening of the distal implant stem would reduce stress shielding and improve proximal load transfer. A cementless size No. 5 Anatomique Benoist Girard (ABG) stem (Howmedica, Allendale, NJ, USA) was merged with a bone finite element (FE) model. Analyses were carried out on the full model and on a model with the distal portion of the stem given an elastic modulus of 1.0 MPa to remove all mechanical contributions. The probability of interface failure was determined using interface stresses (Figure 14), and bone mass development was found through a remodeling simulation.

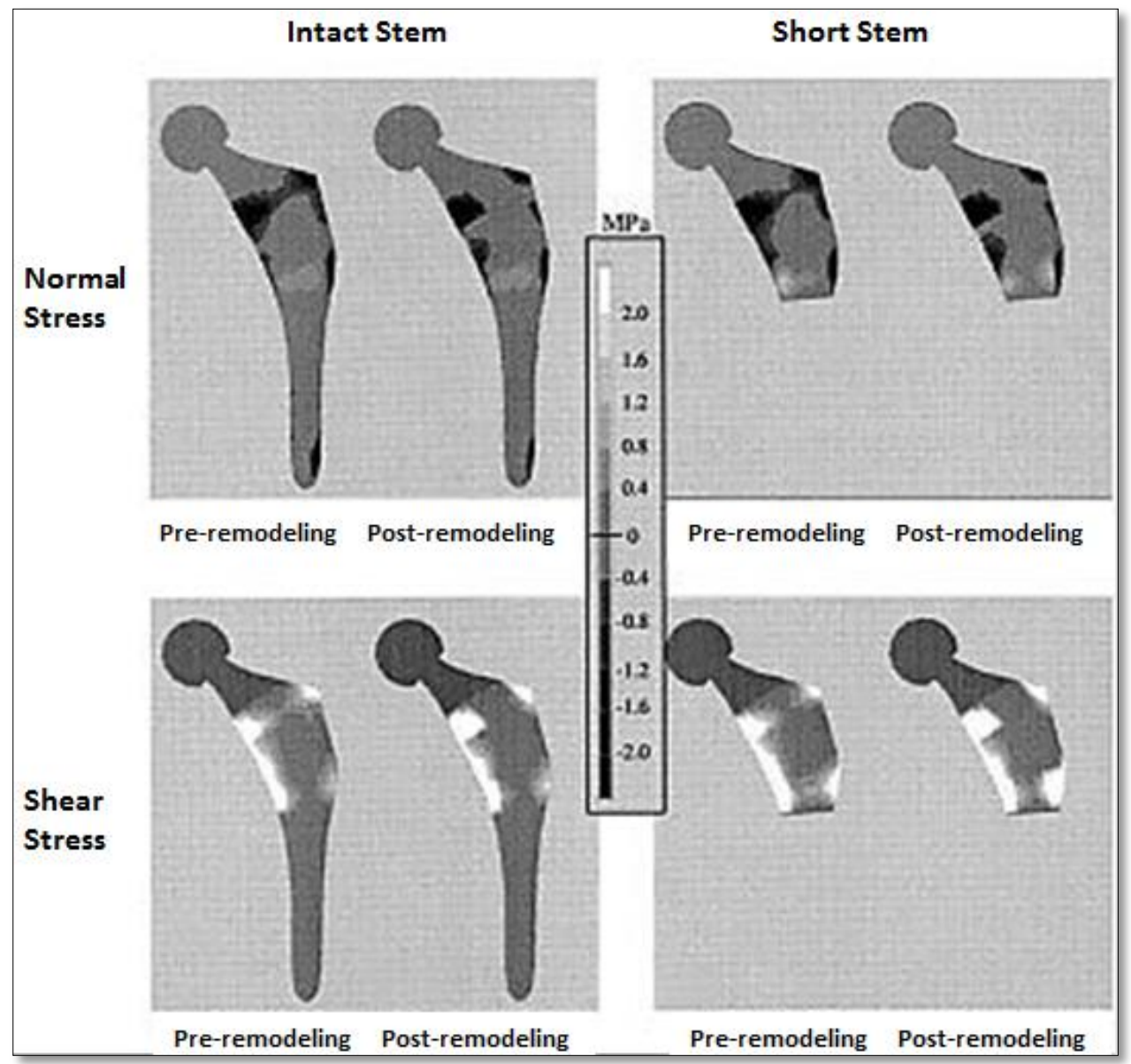

Figure 14: Pre-remodeling and post-remodeling shear stresses in the long and shortstemmed reconstructions, illustrating the implant design and short stem model ${ }^{61}$ 
The ABG short stem was found to provide little benefit in the reduction of stress shielding, transferring stresses more proximally instead of distributing them over the length of the diaphysis. It was also found that the probability for interface failure was very low in both cases, conferring no added benefit to the short stem design. However, while the bone remodeling simulation procedure was validated earlier, the failure criterion was not well supported through experimental validations.

Nevertheless, other literature appraises conservative hip implants much more favourably. Dabirrahmani et al. ${ }^{47}$ undertook an FE study to determine the primary stability and long-term bone remodelling of the BMHR stem. A size 3 BMHR implant was modelled and placed in a CT scanned model femur in the correct surgical position. An intact femur model and the reconstructed models were then subjected to a $45 \%$ gait cycle loading regime, which included muscle loads and the hip contact force. A strain-adaptive remodelling theory was utilized to generate predictive bone remodelling behaviour, from which bone mineral density (BMD) could be derived. Over a predicted period of 30 months, BMD loss occurred only to the proximal neck region, with an increase in BMD at the distal neck and minimal BMD changes otherwise. Some stress shielding was noted only in the proximal neck of the reconstructed femur model, with strain distribution being otherwise comparable to the intact model (Figure 15). This study determined that long-term behaviour of the BMHR was favourable, with minimal BMD changes and good stress distribution. 


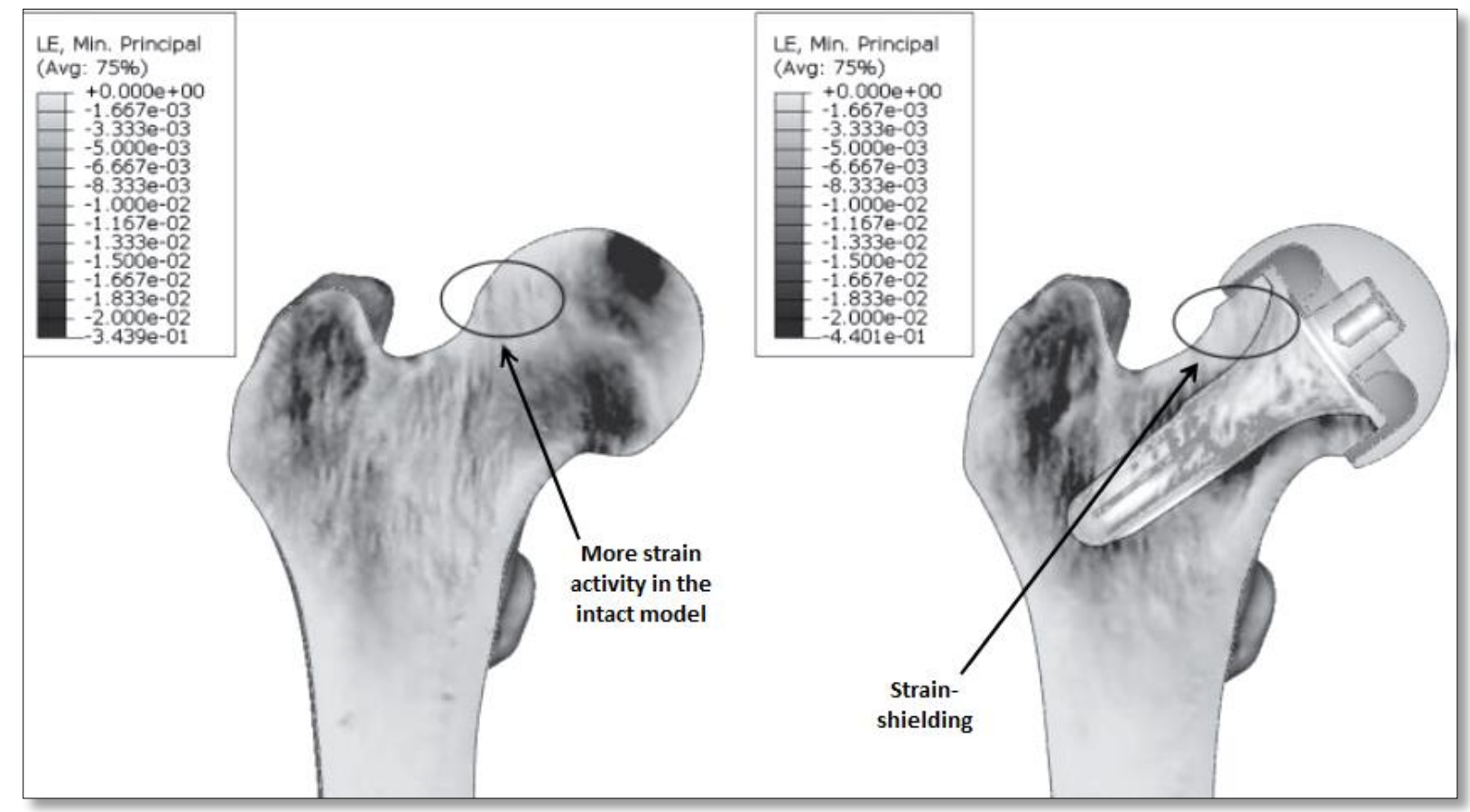

Figure 15: Depiction of compressive strain and strain shielding in the intact and reconstructed femur models ${ }^{47}$

\subsubsection{Experimental Studies}

There are a number of experimental studies that directly compare hip implants with a long stem versus those with a short stem. In a study by Gronewold et al. ${ }^{42}$ synthetic femurs were implanted with the short cementless METHA stem (Aesculap, Tuttlingen, Germany), and a traditional Bicontact stem (Aesculap, Tuttlingen, Germany). Strain gauges were affixed to key locations along the proximal femur, and the implanted femurs were loaded under 6 loading conditions that corresponded to the potential physiological range of hip-joint angles. Recorded strains were taken as a percentage of the measured intact femur strains, and these were then compared between the two stem models. The METHA stem was found to consistently elicit strains similar to the intact model, indicating reduced stress-shielding and good metaphyseal anchorage, while the Bicontact stem had dramatically decreased strains in the calcar region when compared to an intact femur.

In a similar study by Decking et al. ${ }^{31}$ three clinically successful implants - Alloclassic Zweymüller SL (Centerpulse, AG, Winterthur, Switzerland), OPTAN (Centerpulse AG, Winterthur, Switzerland), and CUT (ESKA Implants GmbH \& Co, Luebeck, Germany) - were 
implanted in pairs of human cadaver femurs affixed with rosette gauges. The femurs were loaded up to $2000 \mathrm{~N}$ in single-leg stance and the strains were recorded both before and after implantation. The two traditional stems were found to produce a reduction in principal surface strains in the proximal femur, while the ultra-short stem CUT prosthesis exhibited increased cortical strains in the proximal subtrochanteric region of the femur with otherwise minimal changes (Figure 16). While this indicates that overall proximal stress reduction can be mitigated through use of an ultra-short stem implant, the effect of the increased subtrochanteric strains cannot be determined without long-term clinical follow-up.

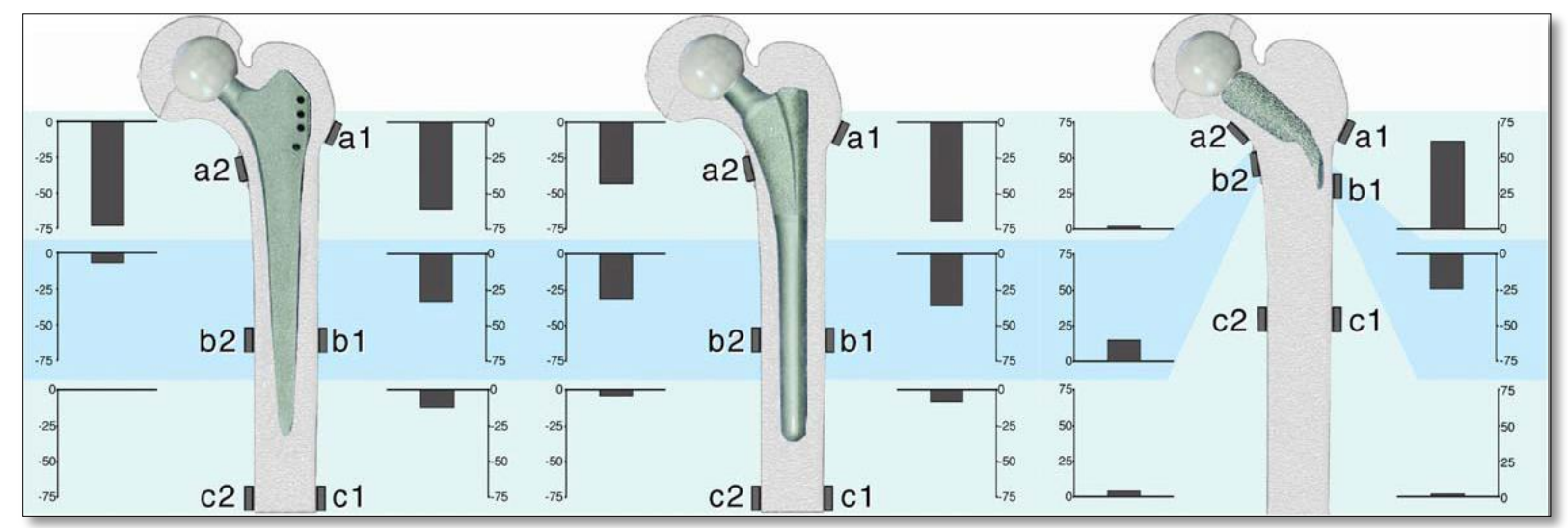

Figure 16: Depiction of the changes in the absolute value of the major principal strains (in $\%$ of the intact femur value) after implantation of the Alloclassic (left), OPTAN (middle), and CUT (right) ${ }^{31}$

Finally, in a study by Bieger et al., ${ }^{36} 9$ pairs of human cadaver femurs were implanted with a long, straight CBC stem (Mathys, Bettlach, Switzerland) and an optimys short stem (Mathys, Bettlach, Switzerland). Inductive miniature displacement transducers were used to measure implant motion over 100000 axial load cycles to simulate 6 weeks in vivo, while rosette gauges were used to measure proximal femur stress. It was found that both implants exhibited primary stability with reversible micromotions less than $150 \mu \mathrm{m}$; however, while both stems also showed stress shielding, the short stem induced a more physiological stress distribution when compared to an intact femur.

\subsubsection{Implant Orientation Studies}

With the development of these new conservative hip implants comes a change in the required surgical techniques and placement goals. For example, Santori et al. ${ }^{25}$ generated a surgical implantation technique for their ultra-short stem implant dubbed "round the corner," which 
involves curving the implant into the femoral neck below the greater trochanter. Surgical implant positioning is another key component of any hip replacement surgery, thereby necessitating studies to determine the optimal placement of this new wave of conservative implants.

Speirs et al. ${ }^{62}$ used an FE model to determine the effect of stem position and orientation on loading of the femur when compared to an intact femur FE model. A Nanos short stem hip implant (Endoplant $\mathrm{GmbH}$, Germany) was virtually implanted into an FE model of a standardized femur to yield 3 different orientations: reference, medialised and anteverted. The reference model was defined by aligning the implant with the femoral joint centre to agree with the intact model, while the medialised model placed the implant $6 \mathrm{~mm}$ medially, $2 \mathrm{~mm}$ posteriorly and $4 \mathrm{~mm}$ superiorly, and the anteverted model rotated the implant about the femoral shaft axis by $7^{\circ}$ to give an $11^{\circ}$ anteversion angle. A previously validated musculoskeletal model was then used to determine muscle and joint contact forces for walking and stair climbing, which were subsequently applied to each implanted model and an intact femur model. Resultant strain energy density was determined for each Gruen zone (Figure 17). Stress shielding was seen in the proximal femur for all implanted models when compared to the intact femur, and was found to be relatively insensitive to implant offset or anteversion. They concluded that surgical misplacement of a short-stemmed implant would have minimal effect on the dispersion of internal loads, and these variations are small when contrasted to the strain distribution in an intact femur. 


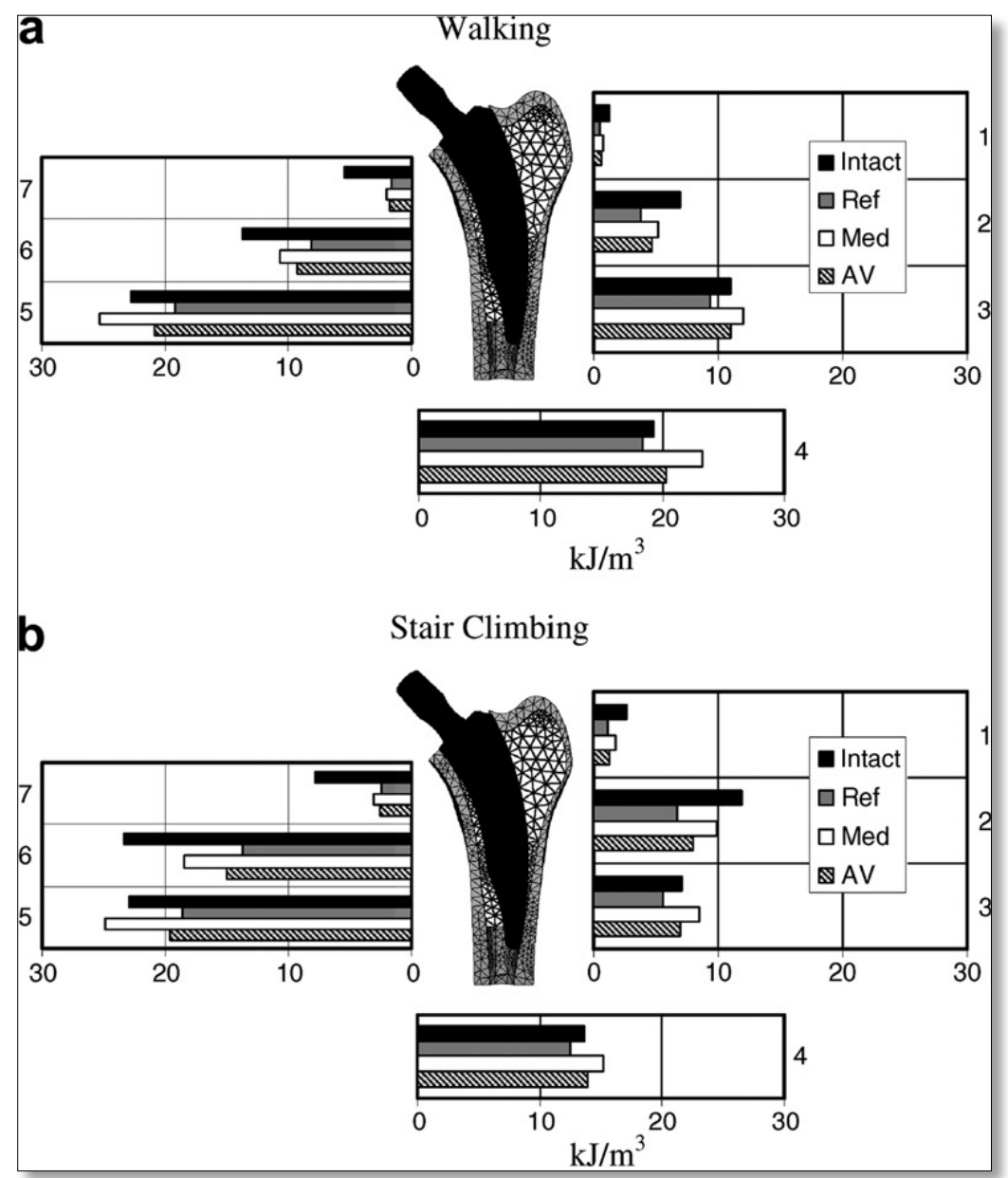

Figure 17: Depiction of cortical strain energy in each Gruen zone for all solid models under walking and stair climbing loads. Intact refers to the intact femur model, Ref is the reference implanted model, Med corresponds to the medialised model, and AV is the anteverted model ${ }^{62}$

Radcliffe et al. ${ }^{54}$ determined the effect of a hip resurfacing stem orientation on load transfer in an intact femur, with a particular focus on the varus/valgus orientation. FE models of 16 subjectspecific CT generated femoral solid models were used, with 4 models per femur: intact, neutral, varus by $10^{\circ}$ and valgus by $10^{\circ}$. The models were then loaded with a joint contact force and an abductor muscle force, and the percentage change in strain between the intact and implanted models was determined. All implanted femurs exhibited some stress shielding in the femoral head (Figure 18); however, the varus implantation angle least resembled the strain distribution seen in the intact femur, as determined through evaluation of the percent change from the intact femur in specified proximal zones. They concluded that the valgus orientation was most comparable to the intact femur, and hence was most desirable. 


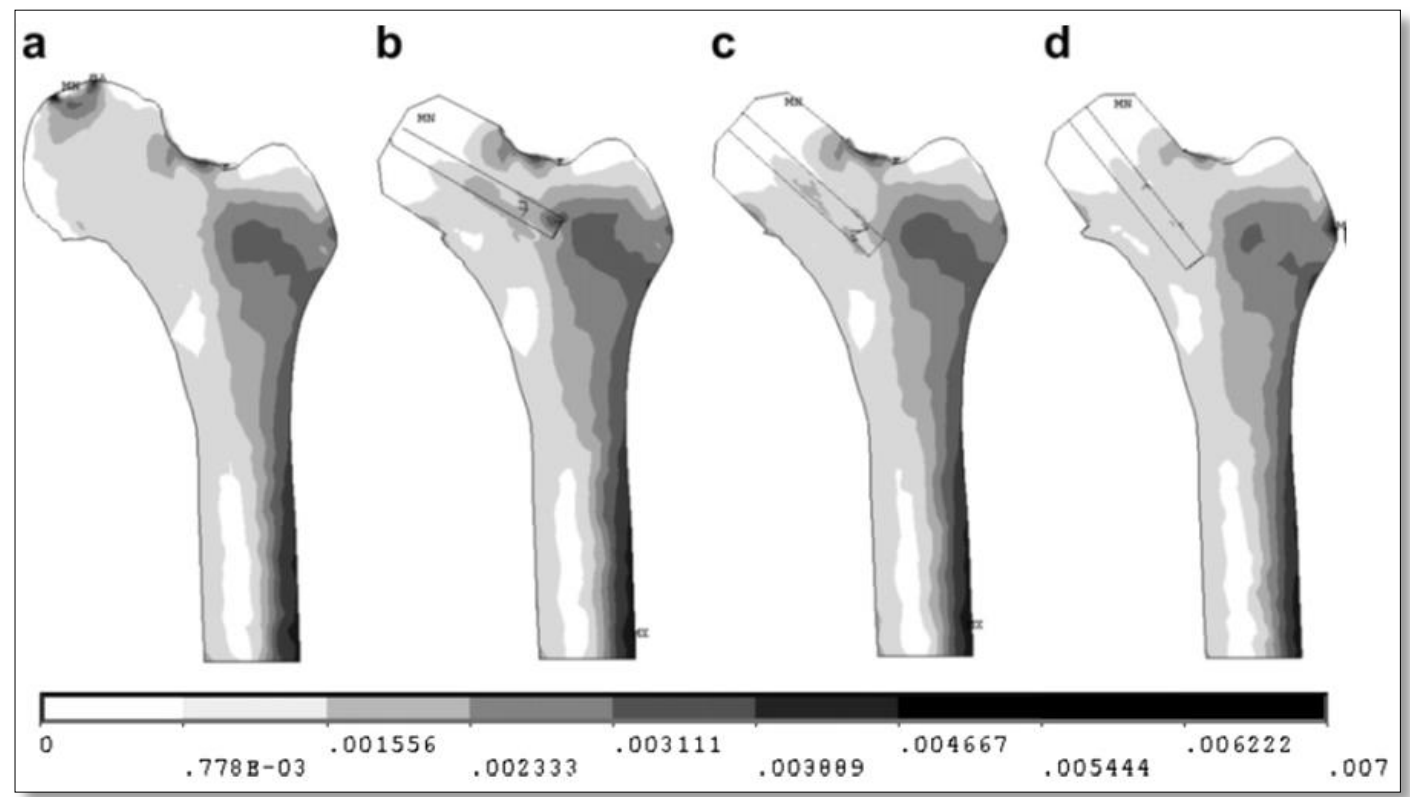

Figure 18: Strain distributions for a single femur model in the: a) intact femur, b) resurfaced in varus, c) resurfaced in neutral, $d$ ) resurfaced in valgus ${ }^{54}$

\subsection{Summary of Findings}

With the increasing prevalence of young patients requiring hip replacement surgery, there has been a corresponding increase in the need for more bone conserving hip implants. ${ }^{19}$ Several reviews have evaluated the conservative implants currently on the market, ${ }^{19,20,44,59}$ while others have specifically examined the physiological and/or biomechanical advantages of conservative implants. $^{25,31,36,40-43,47,48,54,60-62}$ It was determined that ultra-short stem implants have the most favourable stress distributions and hence the most beneficial bone remodeling outcomes, with the added benefits of a less invasive surgical implantation technique and increased bone stock preservation as compared to traditional implants. Surgical placement was also evaluated, and it was shown that a valgus orientation is desirable for hip resurfacing procedures. ${ }^{54}$ However, while the BMHR has been evaluated for its desirable physiological loading characteristics, the new and comparably designed Silent Hip has yet to undergo any biomechanical analysis. In particular, the optimal 3D surgical orientation of such an implant has not yet been studied. 


\section{CHAPTER 5 \\ Research Methods}

\subsection{General Approach}

This is a two part comparison study involving biomechanical testing and finite element modelling. Initially, synthetic femurs implanted with a straight short stem in a specified angle or position were instrumented with strain gauges and mechanically tested in axial compression at a subclinical load of $1000 \mathrm{~N}$. The femurs with a neutrally implanted stem were further tested under extension and flexion conditions in order to capture loading throughout the range of the gait cycle. The strain gauge results of this biomechanical testing were then used to validate an FE model of the implant-femur construct with simulated loading of 1000 N. Design of experiments (DOE) was implemented using the FE model to evaluate the range of potential implant angles and positions, and determine the optimal 3D implant orientation that generated a stress distribution most similar to the intact femur model. This was completed at 3 phases of the gait cycle, and the resultant optimal orientations were compared.

\subsection{Mechanical and Material Properties}

\subsubsection{Synthetic Femurs}

This study utilized 10 large, left, fourth-generation composite femurs (Model \#3406, Pacific Research Laboratories, Vashon, Washington, USA). These synthetic femurs featured cortical bone made using a mixture of short glass fibers and epoxy resin, pressure injected around a solid rigid polyurethane foam cancellous core. ${ }^{63}$ The mechanical and material properties for synthetic femurs can be chosen to fully match the characteristics of a normal human femur, or to partially match the properties of an osteopaenic or osteoporotic femur. Density and strength values corresponding to a normal human femur were selected for this study (Table 2). The densities given for synthetic cortical and cancellous bone both corresponded with human femoral bone properties (Table 1). The mechanical properties have been similarly correlated with human ranges, having exhibited mechanical bending and torsional behaviour comparable to human cadaveric femurs. $^{64,65}$ 
Table 2: Mechanical and material properties for synthetic femur bones ${ }^{63}$

\begin{tabular}{|c|ccccc|}
\hline \multirow{2}{*}{ Bone } & Loading Mode & $\begin{array}{c}\text { Density } \\
\left(\mathbf{g} / \mathbf{c m}^{\mathbf{3}}\right)\end{array}$ & $\begin{array}{c}\text { Ultimate } \\
\text { Strength (MPa) }\end{array}$ & $\begin{array}{c}\text { Young's Modulus } \\
\text { (GPa) }\end{array}$ & $\begin{array}{c}\text { Poisson's } \\
\text { Ratio }\end{array}$ \\
\hline \multirow{2}{*}{ Cortical } & Longitudinal Tension & 1.64 & 106 & 16 & 0.26 \\
\cline { 2 - 6 } & $\begin{array}{c}\text { Longitudinal } \\
\text { Compression }\end{array}$ & 1.64 & 157 & 16.7 & 0.26 \\
\cline { 2 - 6 } & Transverse Tension & 1.64 & 93 & 10 & 0.26 \\
\hline $\begin{array}{c}\text { Cancellous } \\
\text { ("Solid } \\
\text { Type") }\end{array}$ & Compression & 0.27 & 6 & 0.155 & 0.3 \\
\hline
\end{tabular}

The chosen synthetic femurs had a standard geometry with a femoral head diameter of $52 \mathrm{~mm}$, a length of $485 \mathrm{~mm}$, a mediolateral midshaft outer diameter of $32 \mathrm{~mm}$ and a cancellous-filled intramedullary canal diameter of $16 \mathrm{~mm}$ (Figure 19). ${ }^{63}$

Synthetic femurs have been chosen for use in this study as they have a number of benefits over human cadaveric femurs. Human cadaver femurs have large interspecimen variation in geometric and material properties, thereby decreasing experimental reproducibility. By comparison, synthetic femurs have an interspecimen variability 20-200 times lower, providing a much more reliable test sample and a standard geometry for use with accompanying FE models. Furthermore, synthetic femurs have a lower cost, no special storage requirements, and no toxicity. ${ }^{64}$

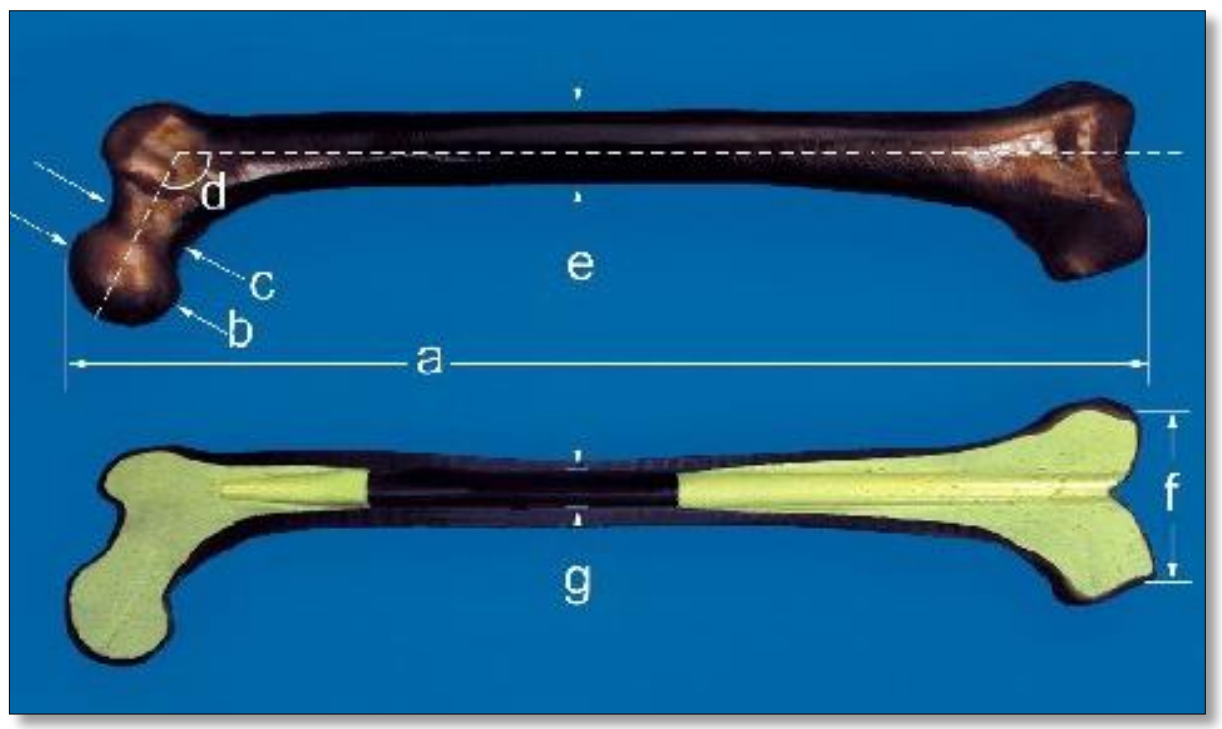

Figure 19: Large fourth generation composite femur and femur cross-section (Model \#3406, Pacific Research Laboratories, Vashon, Washington, USA). Sizing is as follows: a) $485 \mathrm{~mm}$, b) $52 \mathrm{~mm}$, c) $37 \mathrm{~mm}$, d) $120^{\circ}$, e) $32 \mathrm{~mm}$, f) $93 \mathrm{~mm}$, g) $16 \mathrm{~mm}$ canal $^{63}$ 


\subsubsection{Hip Implant Properties}

The hip implant that was chosen for use in this study is the Silent Hip (DePuy, Leeds, UK). Given the dimensions of the synthetic femurs, an optimally sized Silent stem of $22 \mathrm{~mm}$ diameter and 50mm length was selected. The femoral head component consisted of a 36mm 12/14 Articul/eze + 12 ceramic head.

Through scanning electron microscopy (SEM) and energy-dispersive x-ray spectroscopy (EDX), the surface features and material properties of the implant were determined. SEM allowed visualization of the surface of the implant (Figure 20), elucidating the beaded coating on the stem into which it can be assumed that bone would mechanically interdigitate. ${ }^{66}$

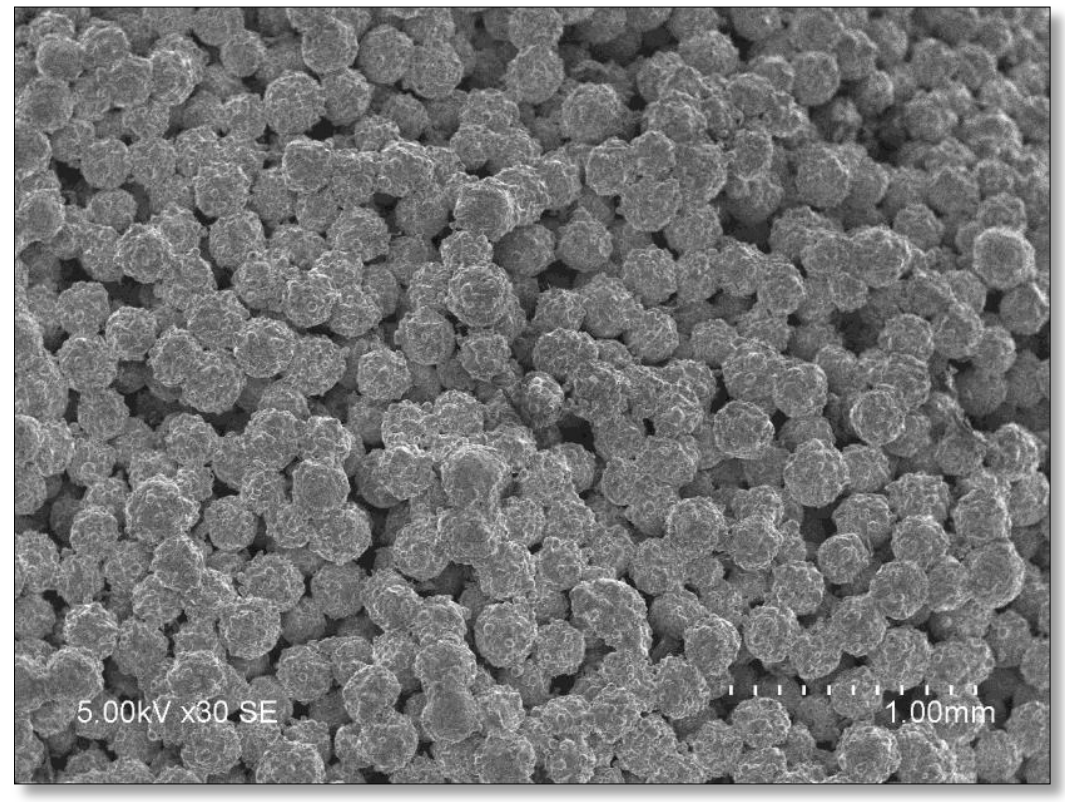

Figure 20: SEM of the Silent Hip stem, depicting the sintered calcium phosphate bead coating at $\mathbf{x 3 0}$ magnification

From EDX it was found that the stem is composed of a titanium alloy, Ti-6Al-4V (Figure 21), with a beaded calcium phosphate coating (Figure 22). 


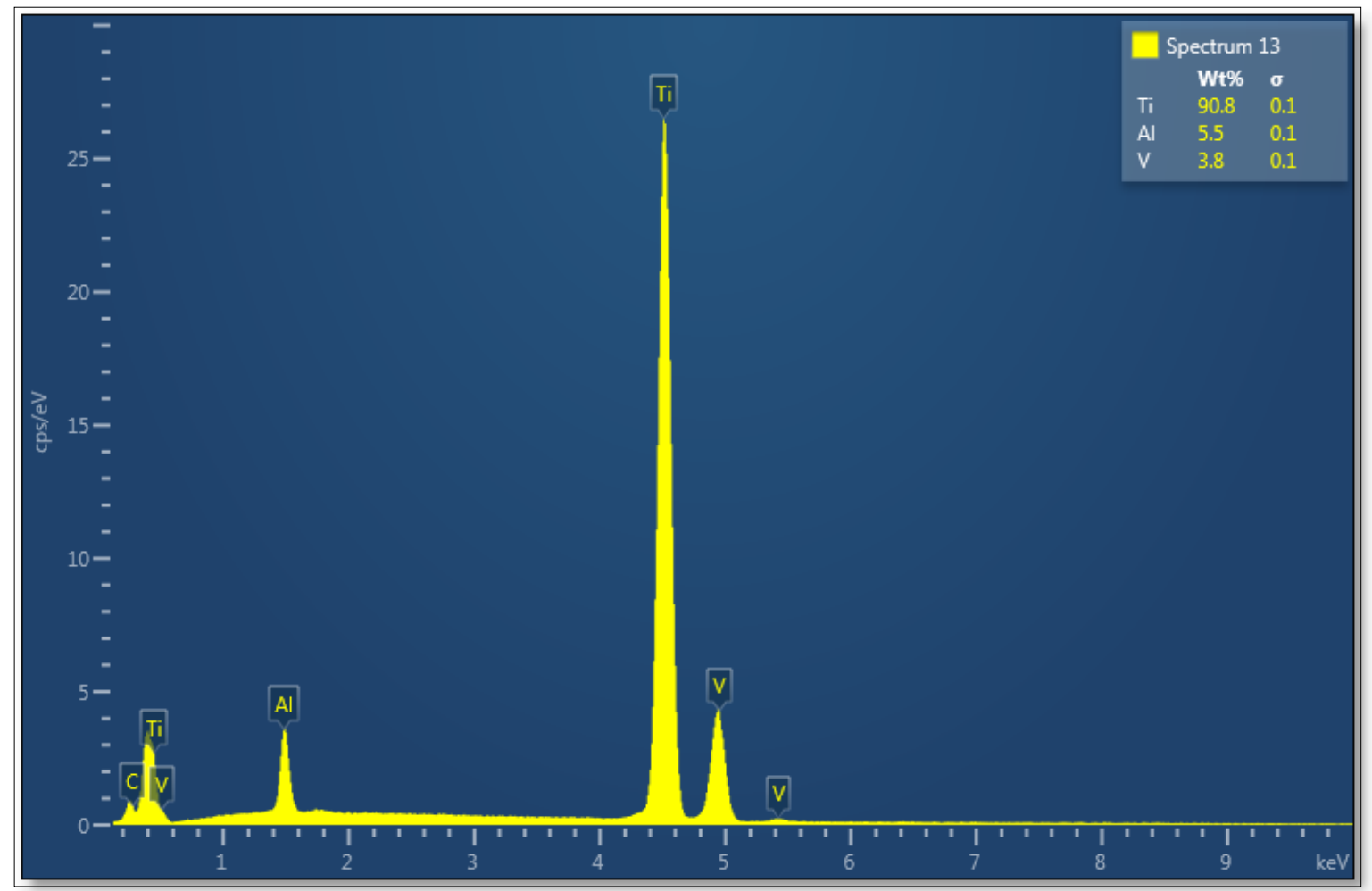

Figure 21: EDX of the stem neck region, illustrating the Ti-Al-V composition of the stem

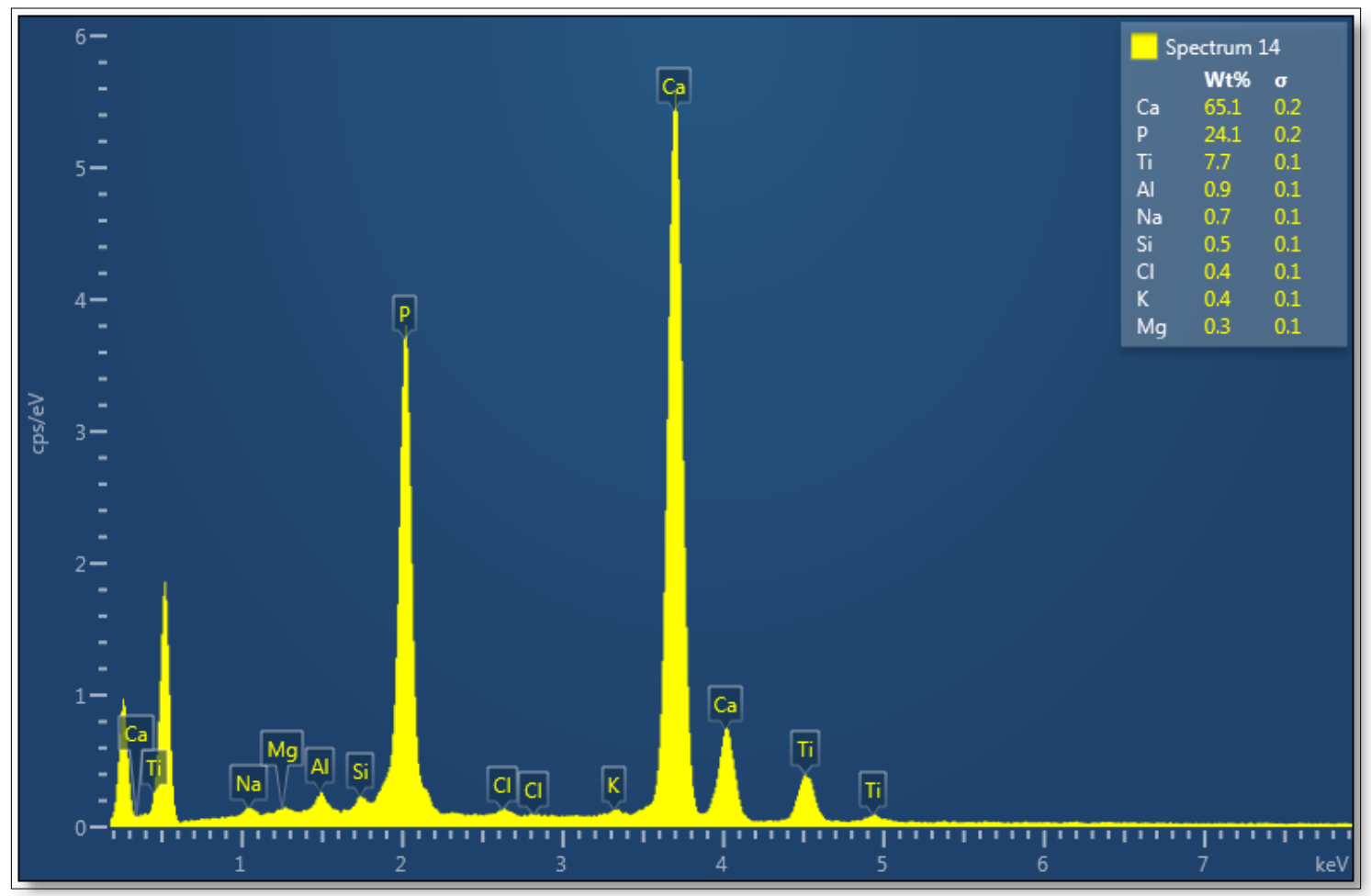

Figure 22: EDX of the stem body region, indicating the composition of the calcium phosphate beaded coating 
The femoral head is comprised of an aluminum oxide matrix composite ceramic called BIOLOX ${ }^{\circledR}$ delta, which consists of $82 \%$ alumina $\left(\mathrm{Al}_{2} \mathrm{O}_{3}\right), 17 \%$ zirconia $\left(\mathrm{ZrO}_{2}\right)$ and additional trace elements such as chromium oxide $\left(\mathrm{Cr}_{2} \mathrm{O}_{3}\right)$, which imparts the pink colour (Figure 23). ${ }^{67}$

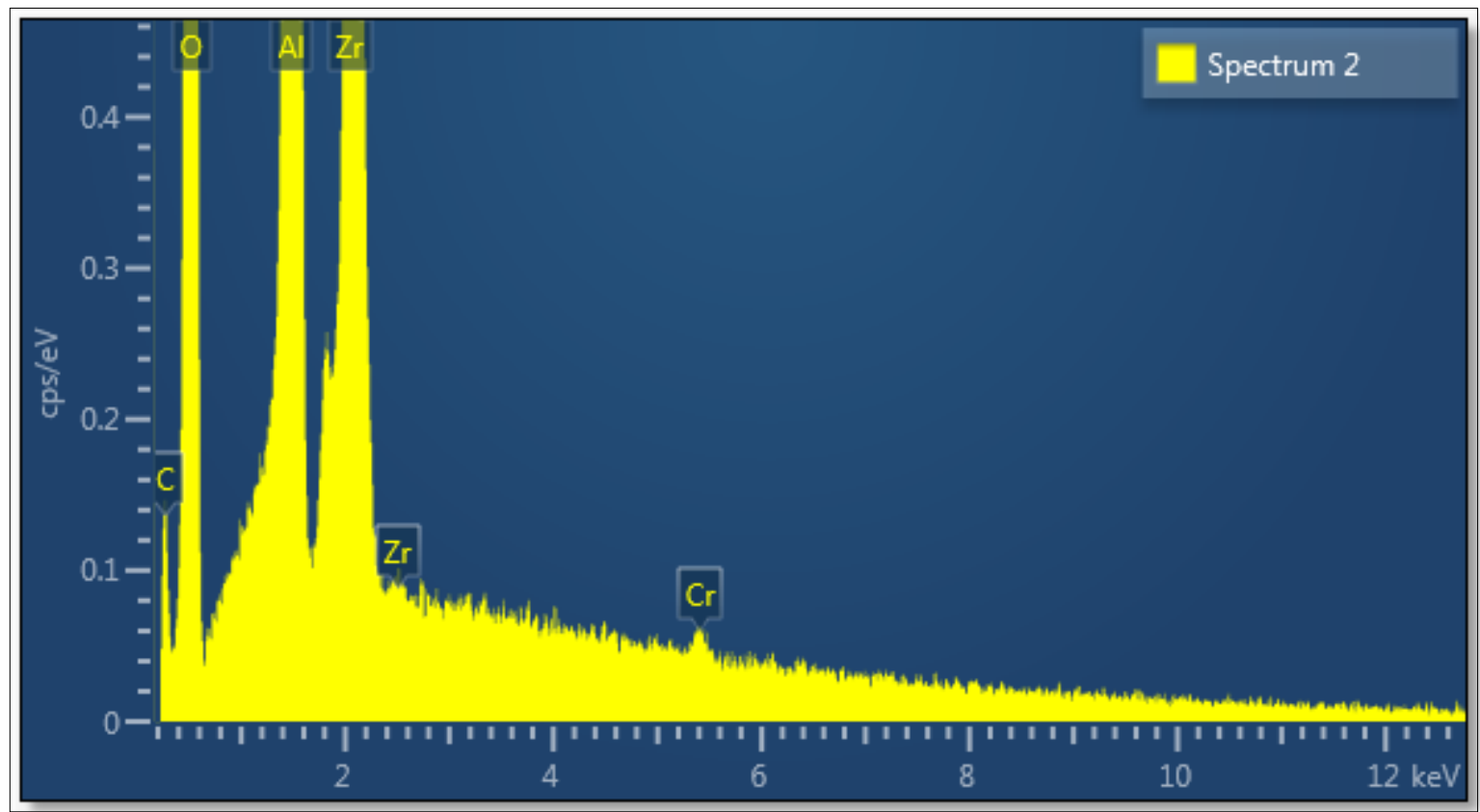

Figure 23: Expanded scale EDX of the ceramic femoral head, indicating the aluminum oxide matrix composition

Due to sintering of the beads used for the calcium phosphate coating, there was a high stiffness modulus throughout the implant. Assuming complete mechanical interdigitation with the implant, it was therefore possible to allow the mechanical properties of the implant to be governed by the bulk properties of the material. ${ }^{66}$ The material properties of the stem were therefore set to those of the titanium alloy from which its bulk is composed. The material properties that were used in the FEA have been further verified with the manufacturer (Table 3).

Table 3: Material properties of the ceramic femoral head and titanium alloy stem ${ }^{68-70}$

\begin{tabular}{|ccc|}
\hline Material & Young's Modulus (GPa) & Poisson's Ratio (v) \\
\hline BIOLOX $^{\circledR}$ delta ceramic (Femoral head) & 358 & 0.24 \\
\hline Titanium alloy (Ti-6Al-4V) (Silent stem) & 110 & 0.31 \\
\hline
\end{tabular}




\subsection{Biomechanical Testing}

The main objective of this section was to understand the experimental strain distribution within the femur at 7 maximum implanted orientations of the Silent Hip, at 3 phases of the gait cycle. These results were then used to validate corresponding FE models of each implant orientation.

\subsubsection{Orientation Definition}

It was determined that 7 experimental implant positions should be tested in order to ensure that the maximum range of clinically realistic potential orientations within the FE model was valid. The stem was therefore implanted in neutral, maximum valgus, maximum varus, maximum anteversion, maximum anterior, maximum posterior, and maximum inferior. Through discussion with an experienced orthopaedic surgeon, it was concluded that a retroverted implant angle or superior positioning would seldom be surgical goals, and hence these orientations were excluded from the current study. ${ }^{71}$ The following maximum orientations were determined experimentally through ascertaining the maximum translation or angulation possible while ensuring the cortical bone was not breached. This ensured that the stem could still be implanted using a press fit, which is required for primary stability. ${ }^{49}$

The neutral orientation was defined as a position that maintained the natural collum-caput-diaphyseal (CCD) angle of the synthetic femur in the anteroposterior (A/P) view (Figure 24), while also maintaining a neutral lateral angle and position (Figure 25). 


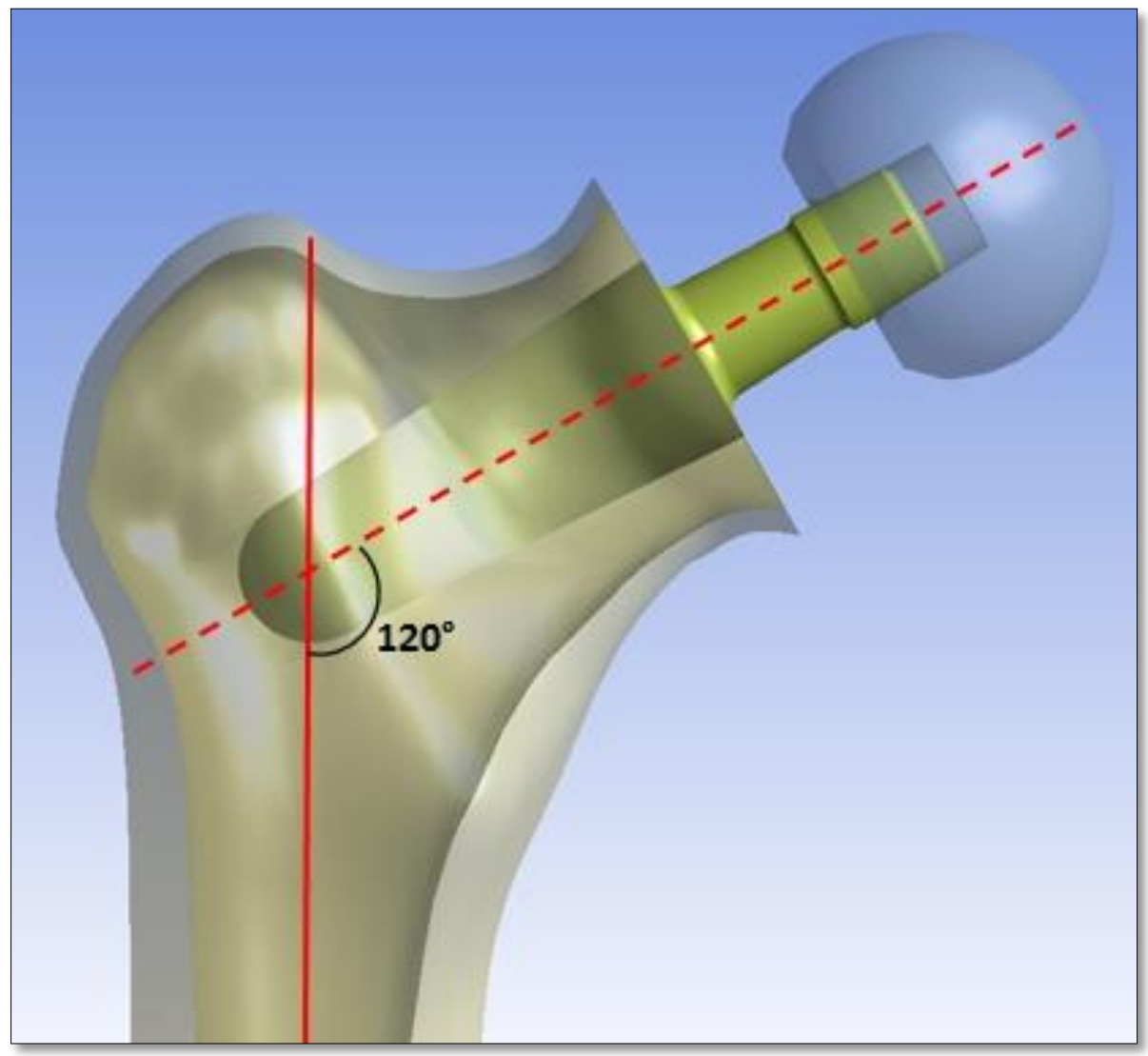

Figure 24: A/P view of the Silent stem neutrally implanted within the femur, where the solid red line indicates the long axis of the femur and the dashed line represents the implant axis at a CCD angle of $120^{\circ}$

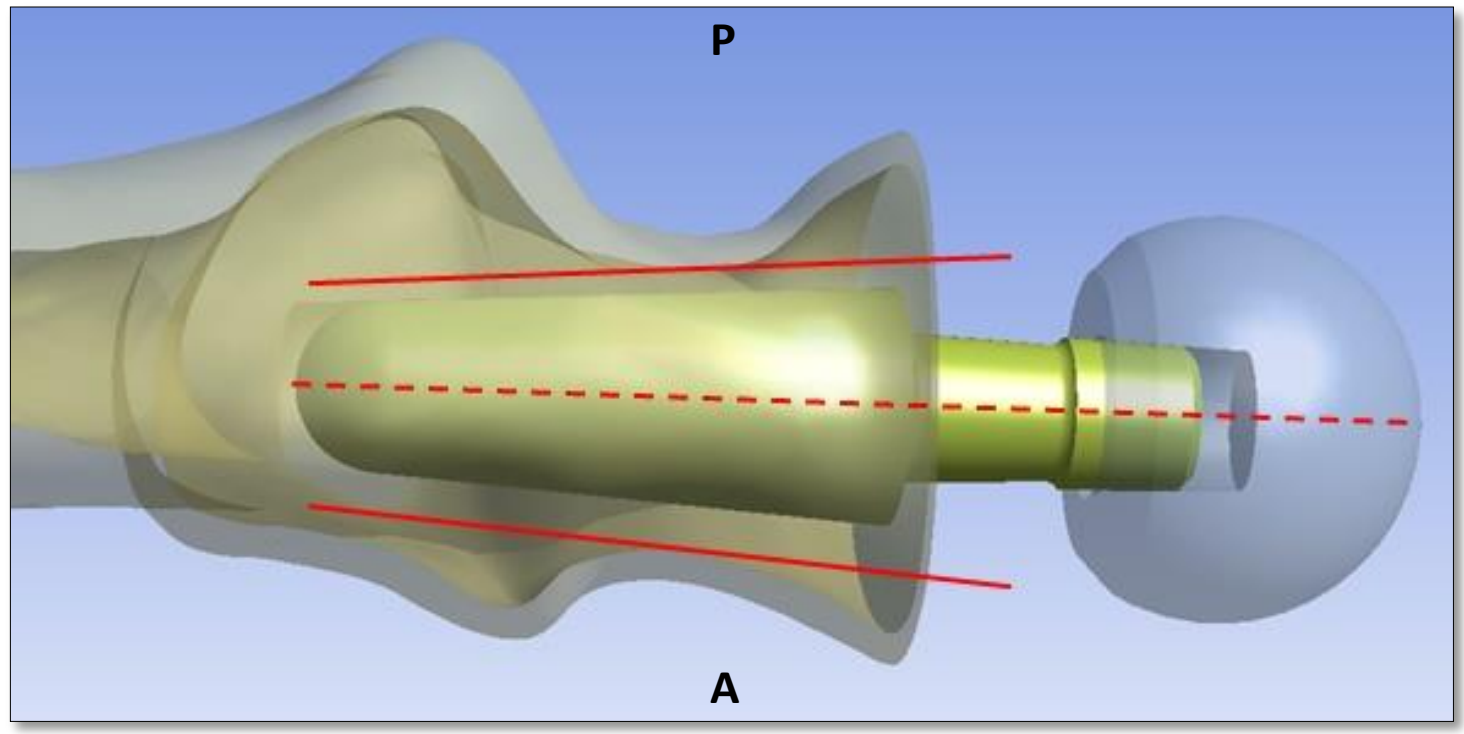

Figure 25: Lateral view of the Silent stem neutrally implanted within the femur, where its neutral angle and position are displayed through its central location between lines tangent to the cortical bone 
The maximum valgus implant orientation was determined to be at a CCD angle of $130^{\circ}$, with a neutrally angled and positioned lateral implant orientation (Figure 26).

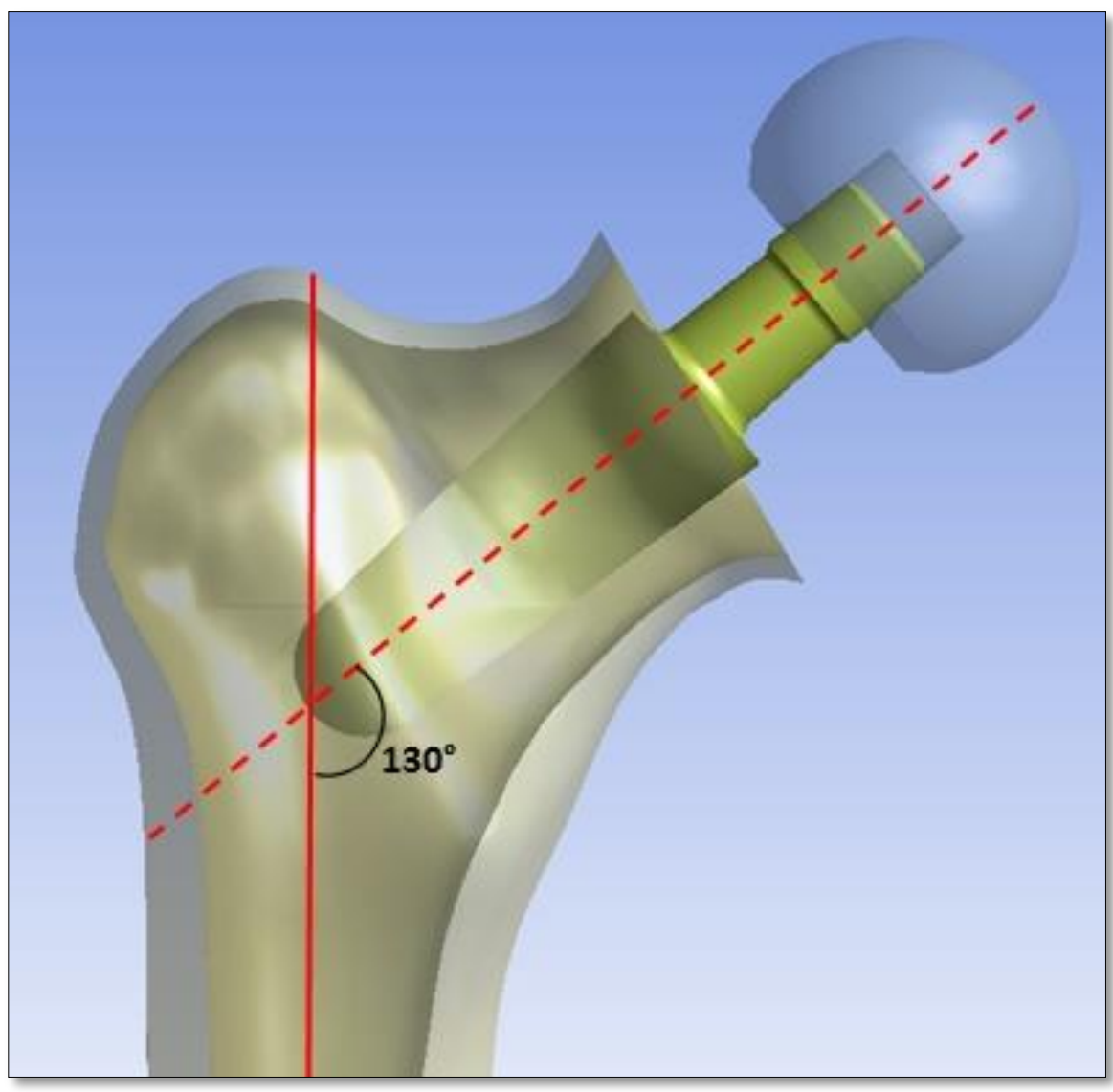

Figure 26: A/P view of a Silent stem implanted in $130^{\circ}$ valgus

The maximum varus orientation was similarly oriented laterally; however, it featured an implant CCD angle of $110^{\circ}$ (Figure 27). 


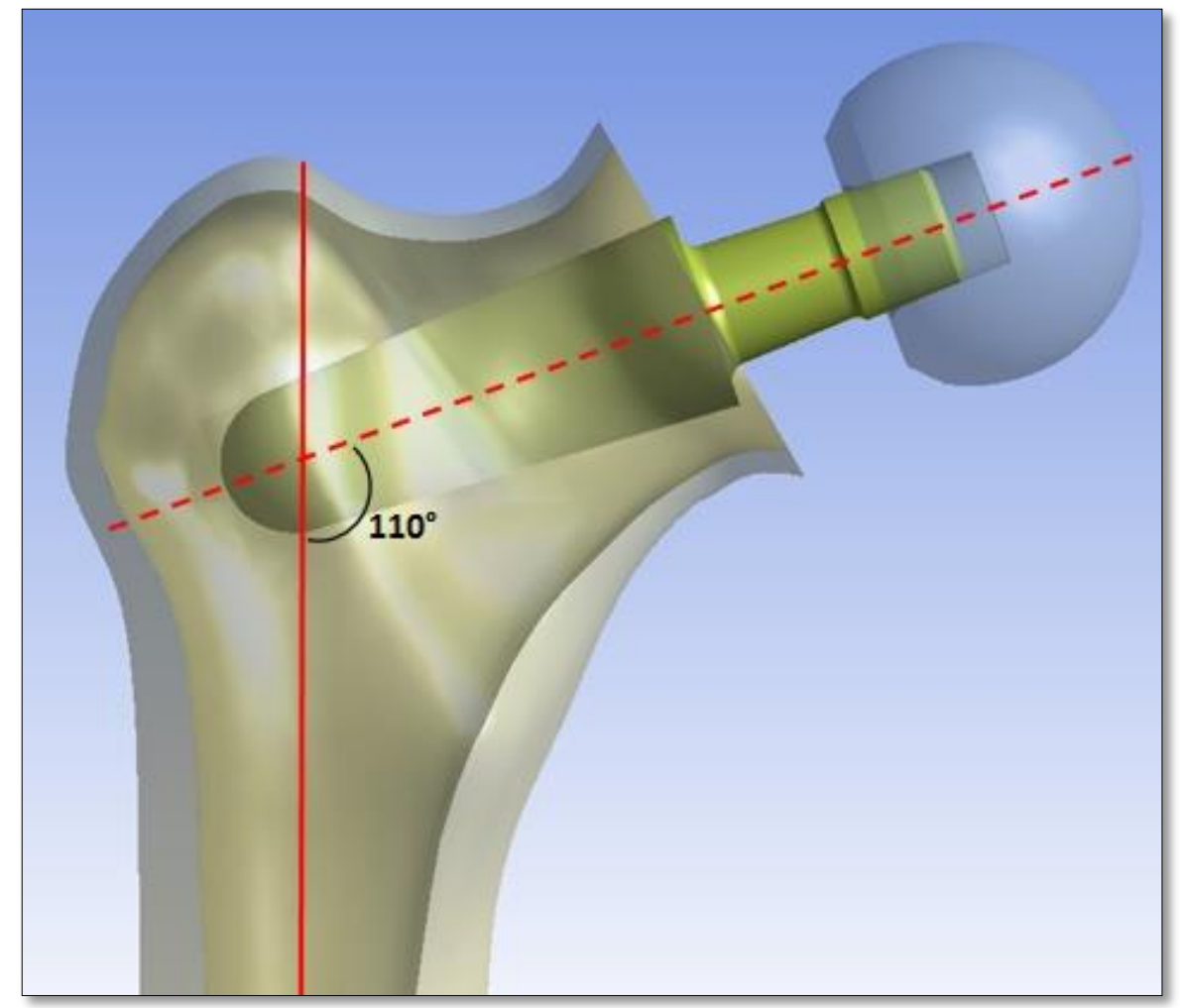

Figure 27: A/P view of a Silent stem implanted in $110^{\circ}$ varus

The maximum anteversion angle was defined as being a stem-neck angle (SNA) of $10^{\circ}$, with a neutral CCD angle (Figure 28).

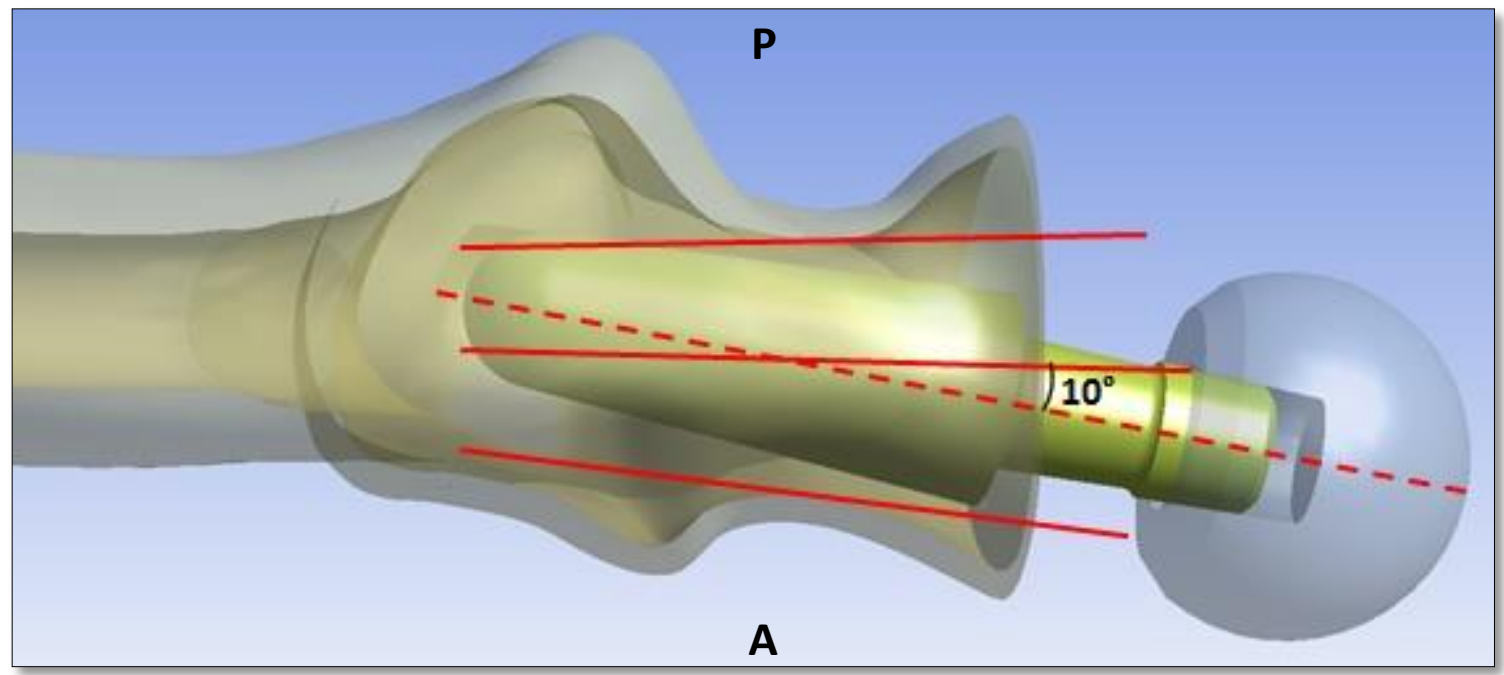

Figure 28: Lateral view of a Silent stem implanted in $10^{\circ}$ anteversion

The maximum anterior position was determined to be a distance of $2 \mathrm{~mm}$ from the neutral line in the lateral view (Figure 29). 


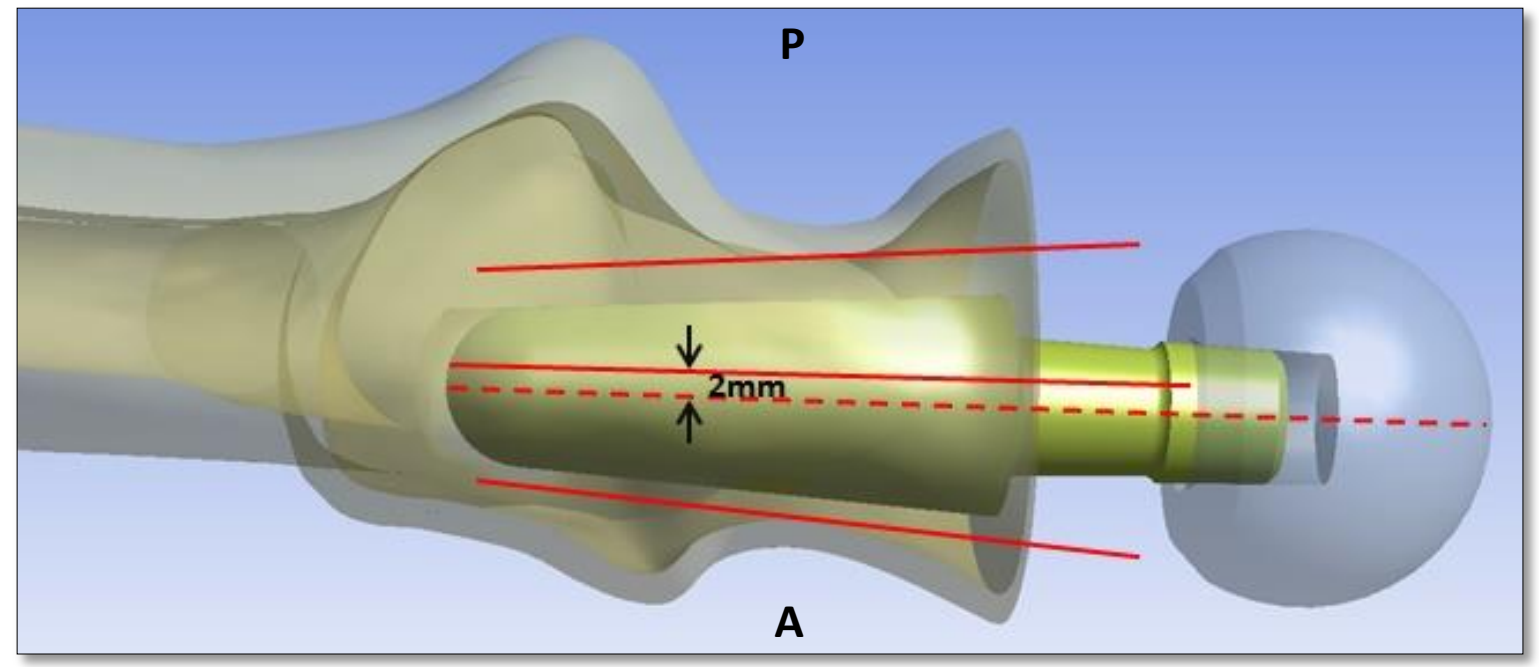

Figure 29: Lateral view of a Silent stem implanted in $2 \mathrm{~mm}$ anterior

The maximum posterior position was similarly found to be at a location $2 \mathrm{~mm}$ from the neutral line in the lateral view (Figure 30).

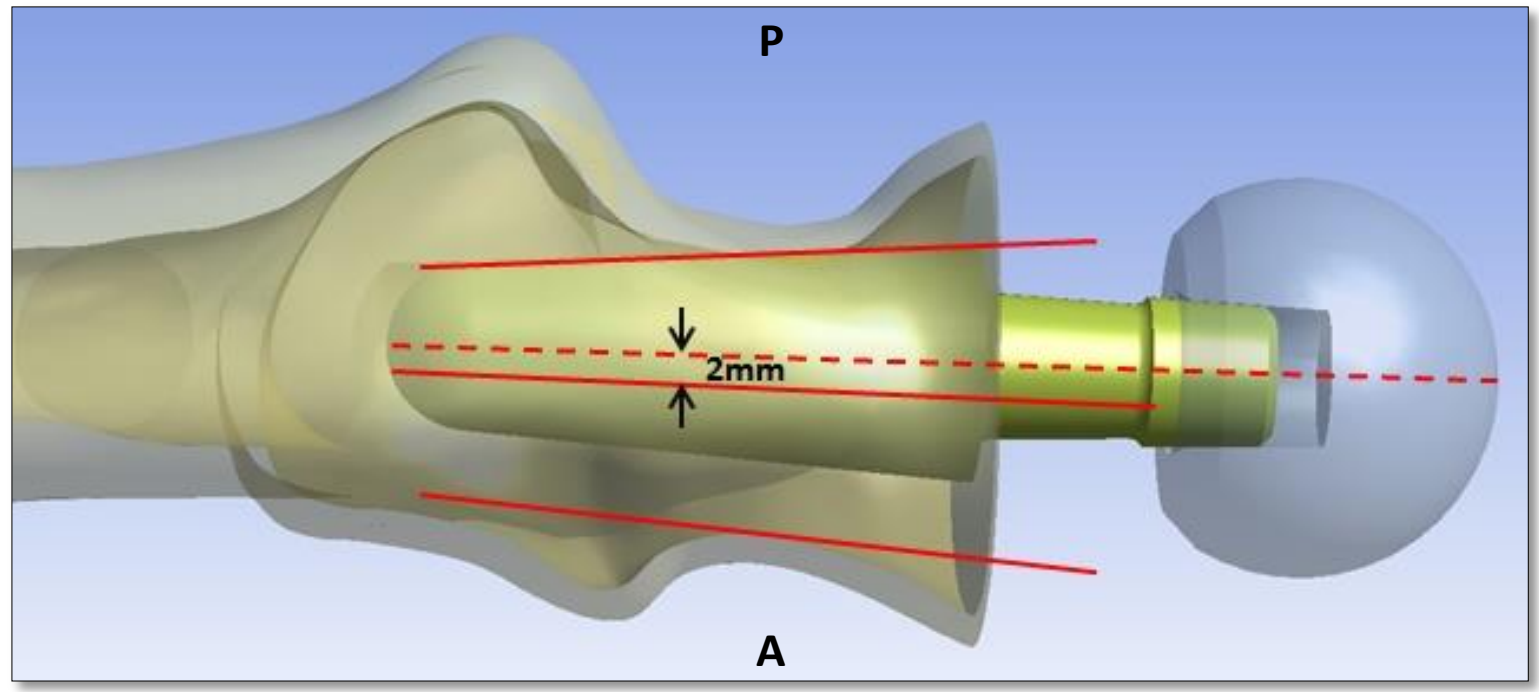

Figure 30: Lateral view of a Silent stem implanted in $2 \mathrm{~mm}$ posterior

Finally, the maximum inferior orientation was found to be located $4 \mathrm{~mm}$ vertically down from the neutral CCD angle line in the A/P view (Figure 31). 


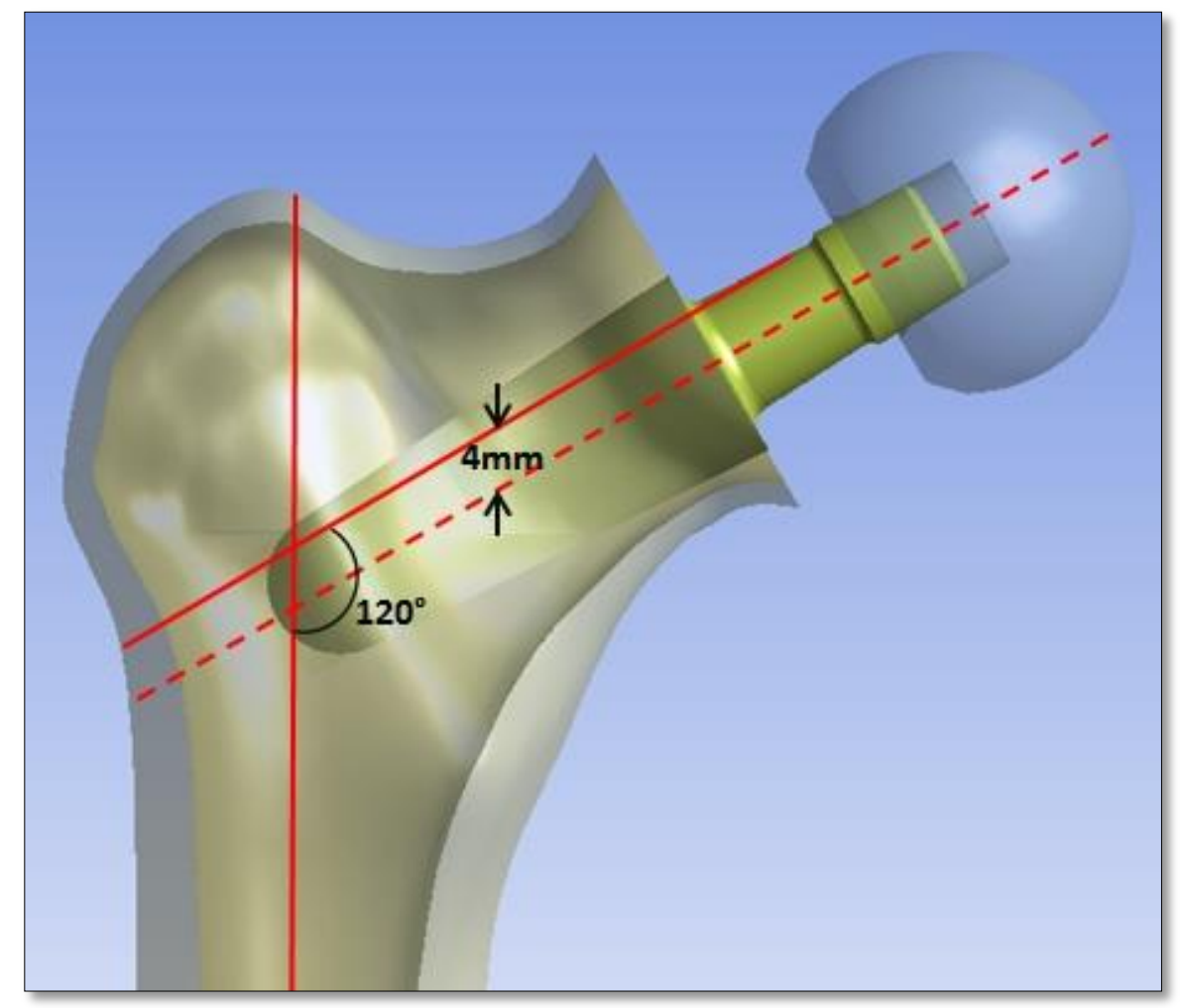

Figure 31: A/P view of a Silent stem implanted in $4 \mathrm{~mm}$ inferior

A summary of the tested angles and positions can be found in Table 4, which details the chosen implant angle or position as described in each view.

Table 4: Planned angles and positions in the A/P and lateral planes for each of the 6 maximum angles and positions, and neutral

\begin{tabular}{|c|c|c|c|c|}
\hline Orientation & A/P Angle $\left({ }^{\circ}\right)$ & A/P Position (mm) & Lateral Angle $\left({ }^{\circ}\right)$ & Lateral Position (mm) \\
\hline Valgus & 130 & 0 & 0 & 0 \\
\hline Varus & 110 & 0 & 0 & 0 \\
\hline Anteversion & 120 & 0 & 10 & 0 \\
\hline Anterior & 120 & 0 & 0 & 2 \\
\hline Posterior & 120 & 0 & 0 & 2 \\
\hline Inferior & 120 & 4 & 0 & 0 \\
\hline Neutral & 120 & 0 & 0 & 0 \\
\hline
\end{tabular}

\subsubsection{Sample Size}

As the biomechanical tests in this study were conducted purely for the purpose of FEM validation, it was determined that only one femur per orientation was required. However, to quantify the repeatability of the surgical implant method, the neutral orientation was implanted in 
3 femurs. Furthermore, an intact femur was prepared and tested to provide a baseline measurement and to allow for validation of an intact model. Overall, there were therefore 10 synthetic femurs used in this study.

\subsubsection{Software-Guided Surgery}

\subsubsection{Alignment of Hip Resurfacing Arthroplasties}

In order to get the degree of implanted accuracy required by this study, computer-assisted navigation was used in place of a goniometer for initial guidewire placement. This method has been shown to greatly decrease the error in guidewire placement when compared to conventional instrumentation during hip resurfacing. ${ }^{72,73}$ Software-guided surgery is often used for implant positioning in hip resurfacing arthroplasties, which feature a femoral component with a small straight stem attached to the femoral head (Figure 32). In typical Birmingham Hip Resurfacing (BHR) (Smith \& Nephew, Marl, Germany) arthroplasties, a $2.4 \mathrm{~mm}$ guidewire is initially inserted into the femur along the planned surgical path, following which a reamer is used to enlarge the hole in preparation for implant placement. ${ }^{74}$ Due to its similarly straight shape and a matching initial reamer size of $8 \mathrm{~mm}$, it was possible to use the BHR software-guided surgery system for initial orientation of the Silent stem.

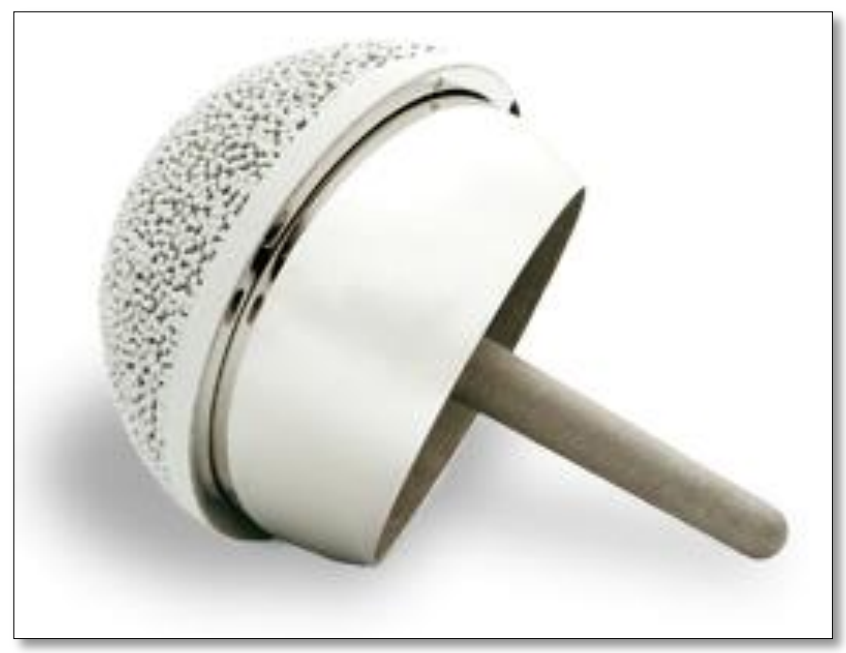

Figure 32: Depiction of the Birmingham Hip Resurfacing (BHR) implant (Smith \& Nephew, Marl, Germany) ${ }^{75}$

\subsubsection{BrainLAB System for Imageless Navigation}

Guidewire placement for orientation of the Silent stem was completed using the BrainLAB Hip Essential system (BrainLAB, Feldkirchen, Germany). This system utilizes two infrared cameras 
to triangulate the location of passive reflective markers in a process called optical motion capture. Initially, an optical array of 3 calibration markers was attached to the femur at the lesser trochanter using a $5 \mathrm{~mm}$ threaded pin (Figure 33) ${ }^{74}$ This established a global coordinate system for localization of the femur and surgical tools in 3D space.

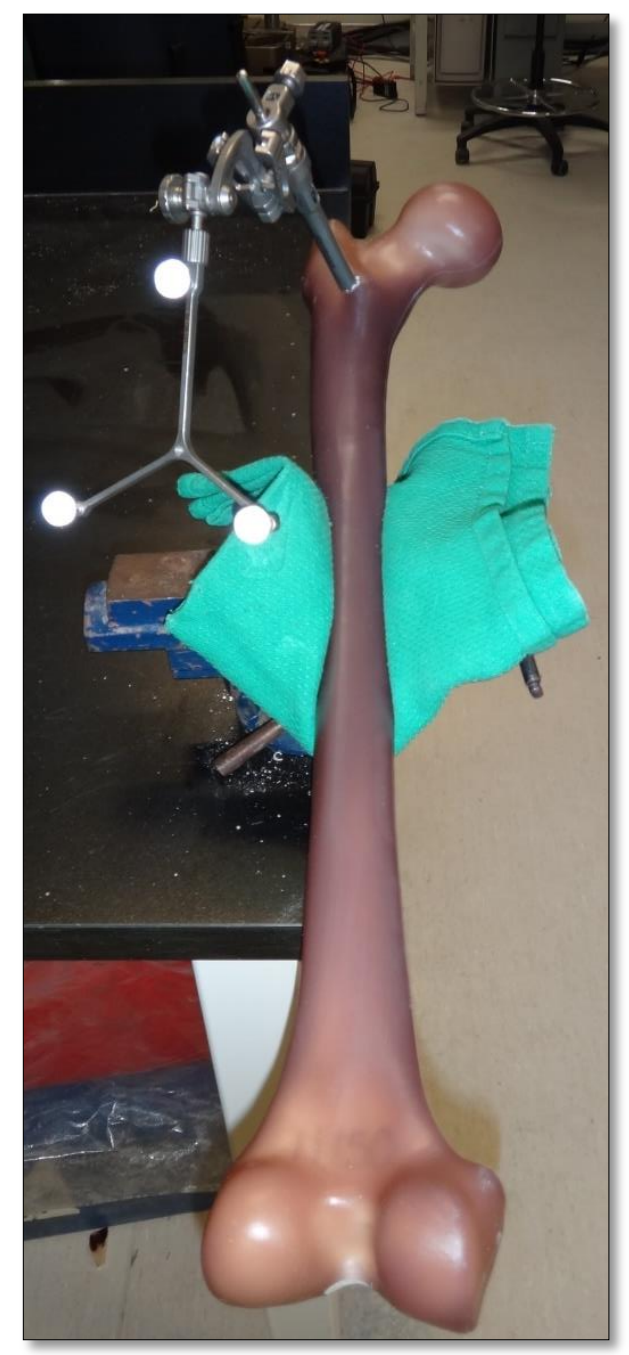

Figure 33: Depiction of an optical array of calibration markers attached to the intact femur The femur size was then registered using an angled pointer equipped with a marker set, which enabled identification of four particular landmarks: the medial and lateral epicondyles, the piriformis fossa, and the head-neck junction. ${ }^{74}$ Points around the femoral head were also taken to establish the femoral head size. Through precise initial calibration, the femur size and location can be established to within $\pm 1^{\circ}$ of accuracy. Furthermore, following registration of the femur, guidewire placement can be tracked in real-time. 
The BrainLAB software automatically generates a surgical plan based on the initial registration, which can then be modified according to user requirements. It was therefore possible to move the guidewire placement to a location or angle corresponding to each of the predetermined orientations. Within the surgical planning screen, an $\mathrm{A} / \mathrm{P}$ section allowed modification of the CCD angle and superior/inferior implant position, a lateral section enabled alteration of the version angle and anterior/posterior placement, and finally a section that included a cross-section of the femoral neck allowed verification of neutral alignment (Figure 34).

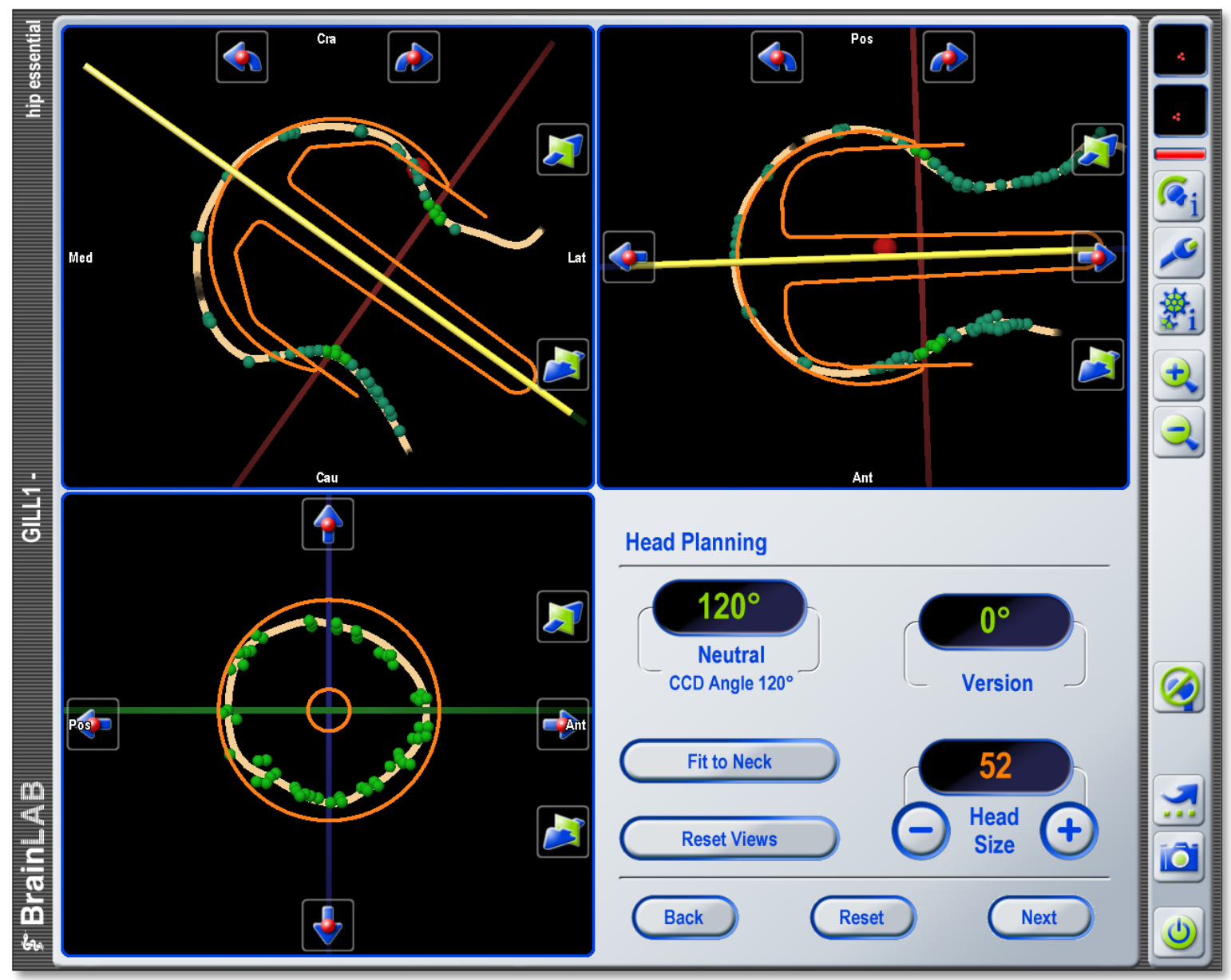

Figure 34: Screenshot of the surgical planning screen provided by the BrainLAB Hip Essential software

Once the desired surgical path had been selected, the guidewire was drilled into the femur through a drill guide, which was equipped with a marker set to allow for alignment of the guidewire along the planned path (Figure 35). 


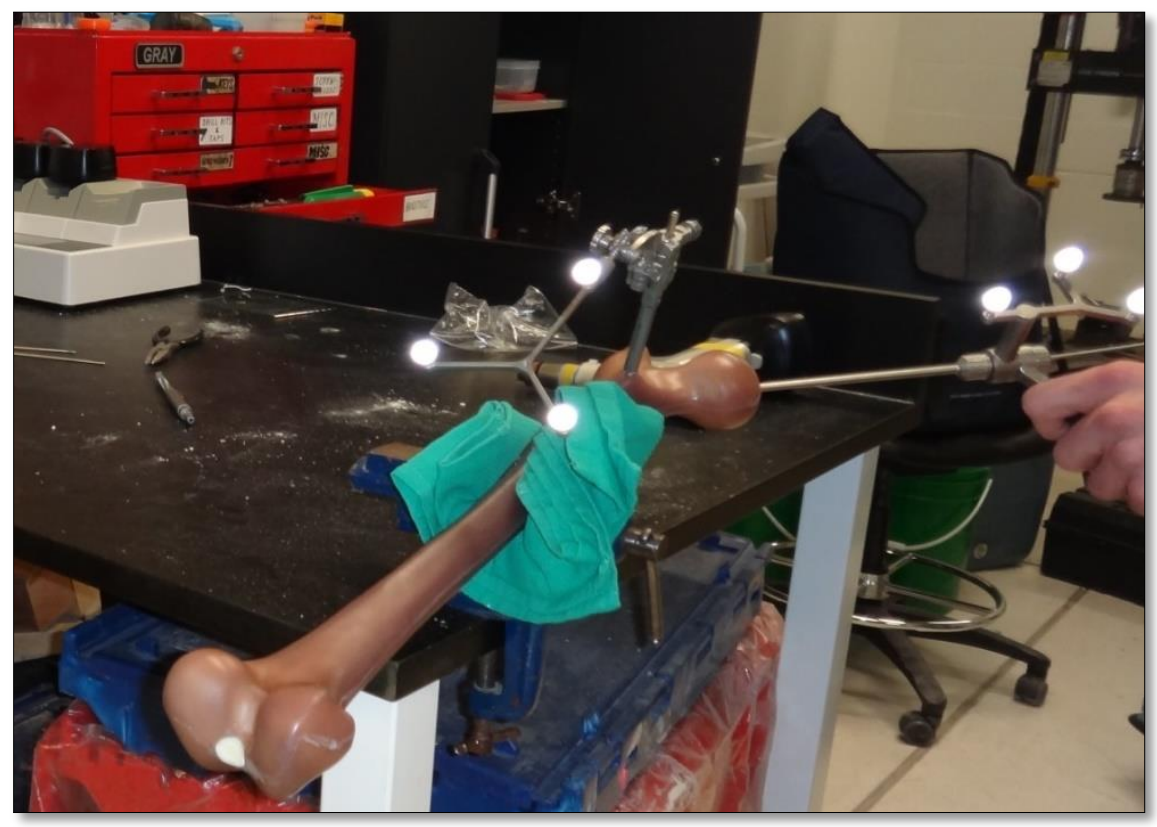

Figure 35: Image of the registered femur undergoing guidewire placement using a drill and marker-equipped drill guide

A real-time feedback system on the computer screen ensured that the guidewire was drilled to within $\pm 1^{\circ}$ of accuracy of the planned surgical path. Once the drill had been placed to an adequate depth within the femur, a verification screen was presented which indicated both the planned and achieved surgical paths and the difference between the two (Figure 36). This provided confirmation of the guidewire placement accuracy. 


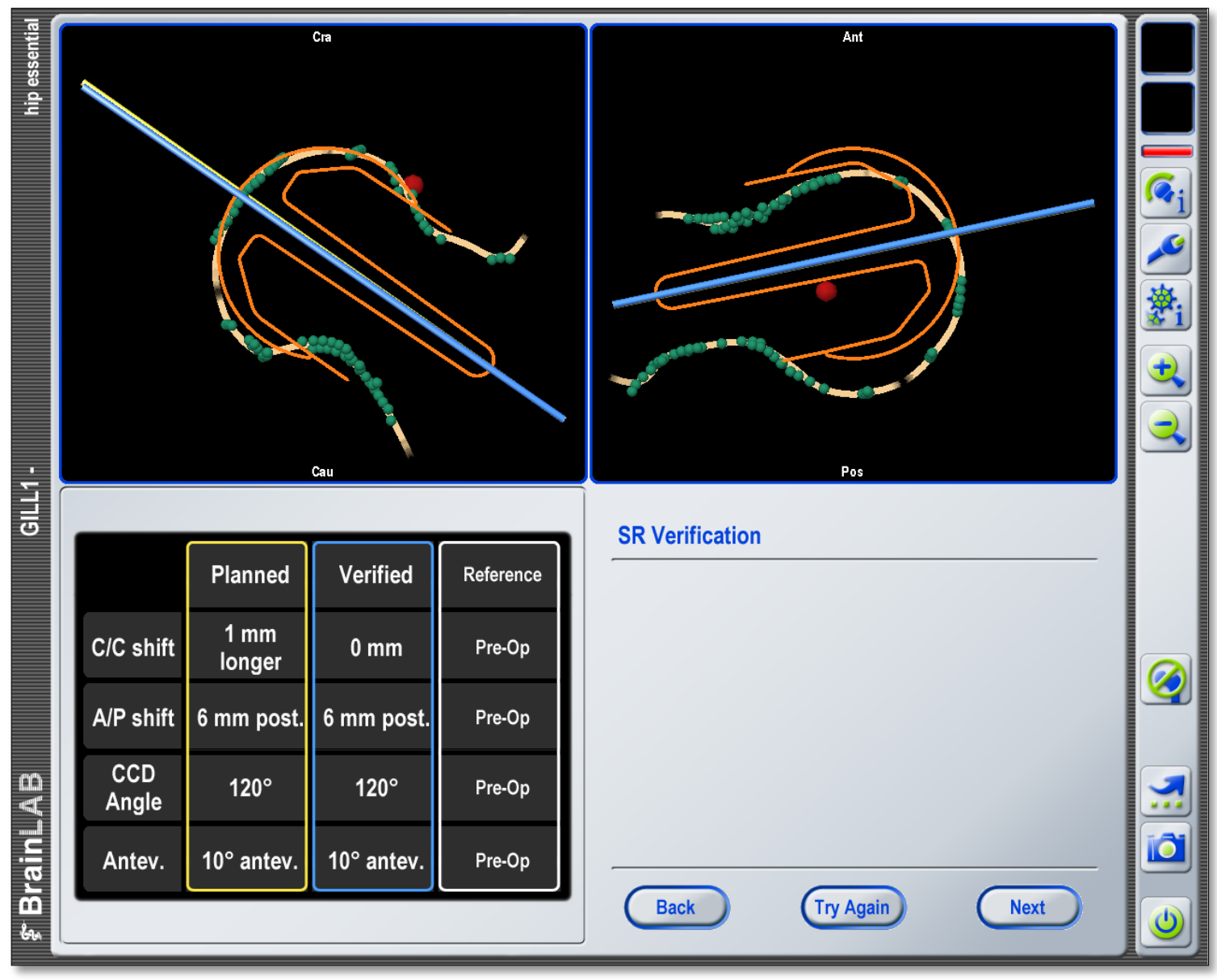

Figure 36: Screenshot of the planned and achieved surgical paths for a neutrally aligned guidewire

\subsubsection{Silent Stem Implantation}

Following guidewire placement for each of the 7 planned orientations (including 3 placements in neutral), a cannulated reamer from the BHR surgical set was used to ream overtop of the guidewire and enlarge the initial cavity. The Silent Hip surgical technique was then followed in order to implant the Silent stem. ${ }^{76}$ Following head resection, this technique utilizes sequential reamers in order to systematically ream the implant cavity to the desired diameter, without significantly altering the planned orientation (Figure 37). 


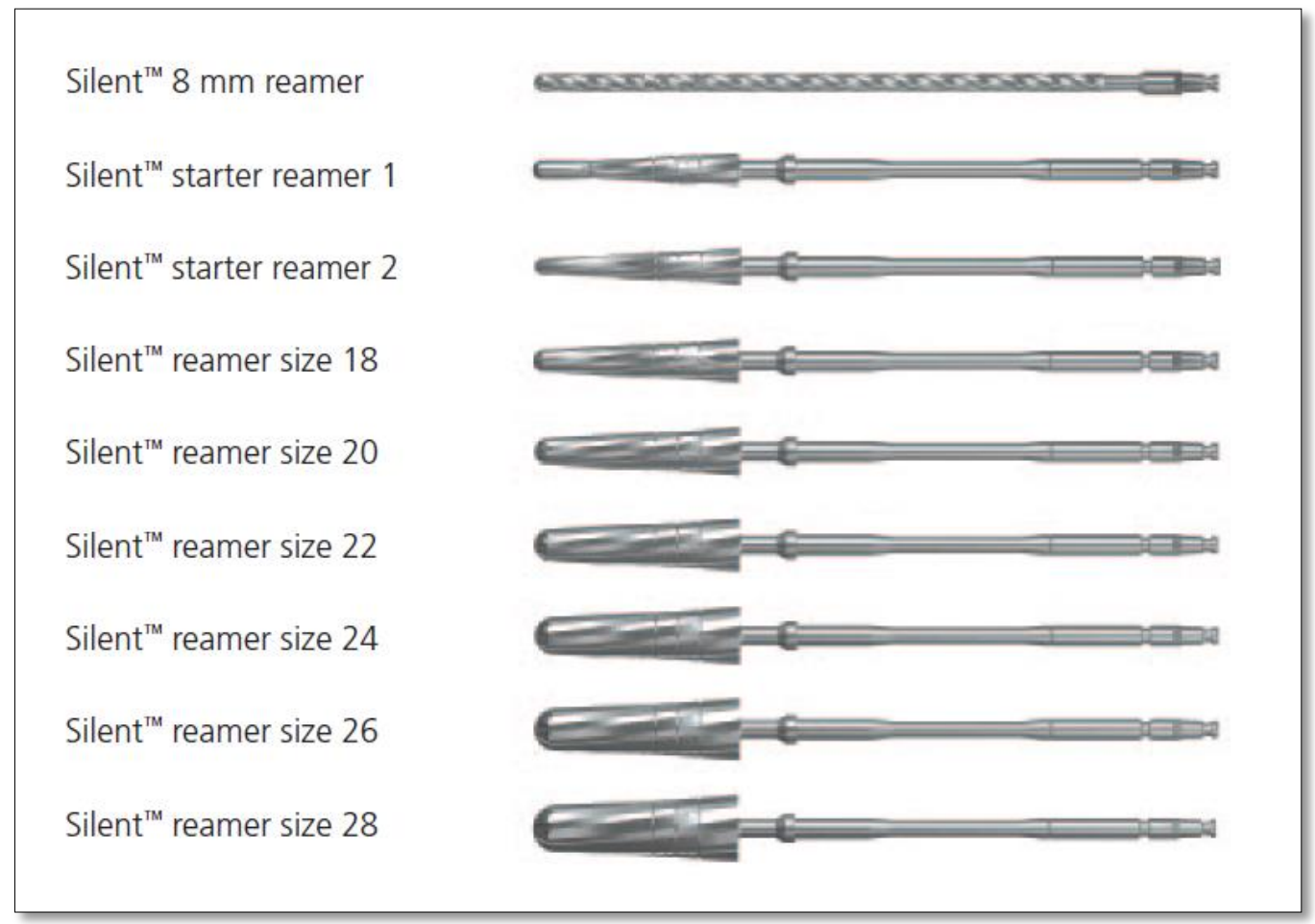

Figure 37: Reamers included in the Silent Hip surgical technique

A depth arm in addition to a depth gauge can be utilized to ensure reaming to an adequate depth for the intended implant. In the Silent Hip surgical technique, it is recommended that the final depth be greater than the implant length, as the implant should sit proud of the neck isthmus by $3 \mathrm{~mm}$ and should include a $3 \mathrm{~mm}$ distal gap (Figure 38). This ensures optimal implant support proximally, and reduces the potential for distal loading. ${ }^{76}$ 


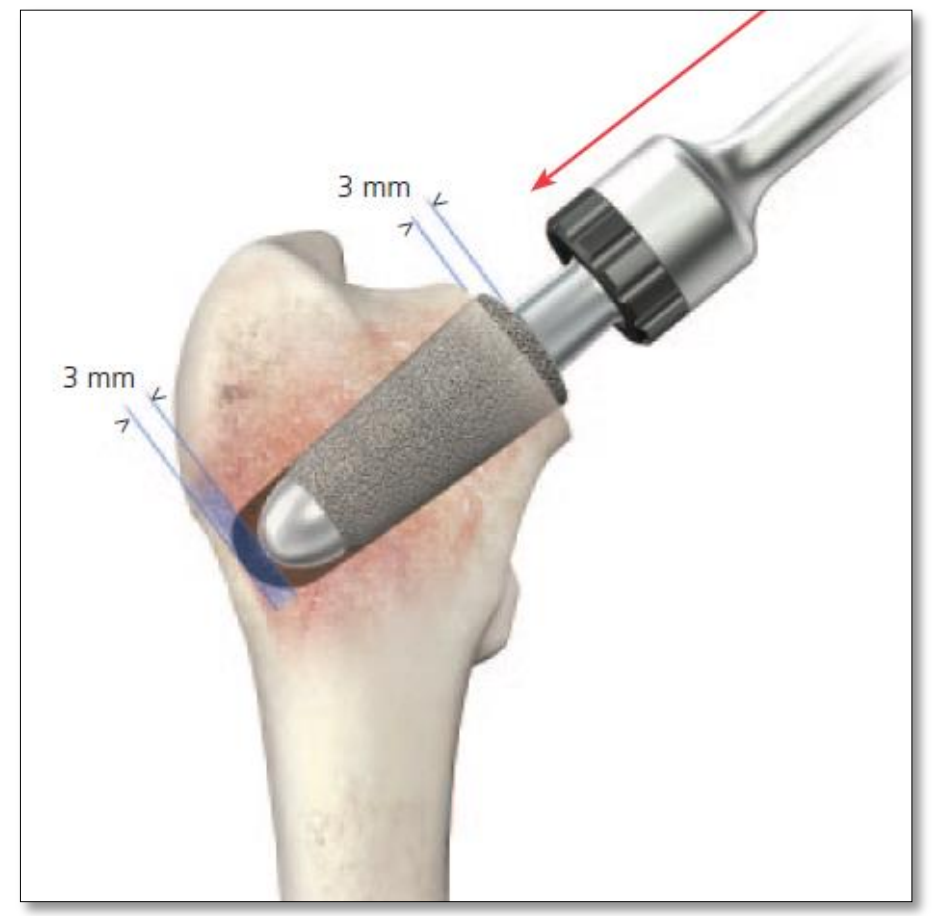

Figure 38: Silent stem placement using the implant impactor instrument, indicating the final seating height and the $3 \mathrm{~mm}$ distal gap

However, the femurs used in this experiment had to be uniformly resected in order to allow for a single validated FE model. It was therefore not possible to use the calcar mill to ensure a final resection plane at the angle of the implant. The femurs were therefore uniformly cut along a resection line located at approximately $38 \mathrm{~mm}$ from the proximal tip of the femoral head, and at an angle of $30^{\circ}$ from the longitudinal axis of the femur (Figure 39), which was determined to be a conservative resection line for a neutrally implanted stem. At this point, the distal condyles of the femur were also removed to allow for subsequent distal fixation (Section 5.3.6.1). For this study, the implant cavity was reamed to a diameter of $22 \mathrm{~mm}$, and a depth of $50 \mathrm{~mm}$. The Silent stems were then impacted to a point at which no portion protruded more than $3 \mathrm{~mm}$ from the resection line. Given that the surgical technique recommends only an approximate final seating height of $3 \mathrm{~mm}$, it was not expected that either the protrusion of the stem or the inclination of the final resection plane would have a significant impact on the final results. 


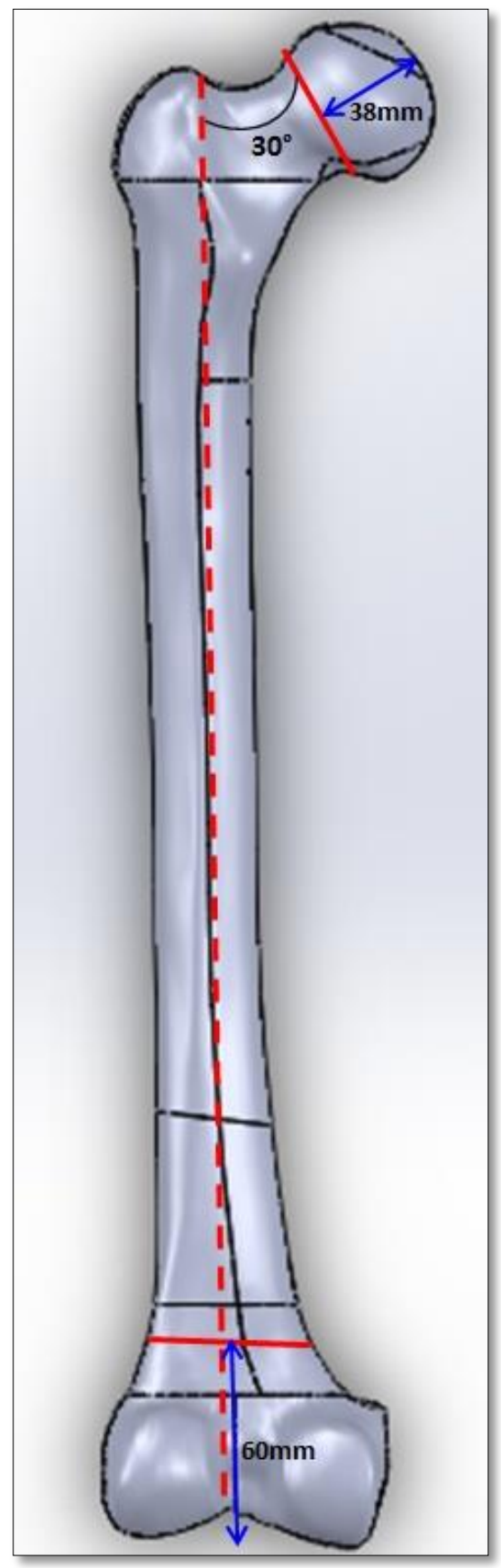

Figure 39: Model of the intact femur, where the solid red lines indicate the respective resection lines and the dashed line indicates the longitudinal axis of the femur

\subsubsection{Orientation Verification}

Implant placement was verified through plain film radiographs of the $\mathrm{A} / \mathrm{P}$ and lateral positions of the implanted femurs. This was completed following guidewire placement to initially confirm the desired orientation, and again following implant placement to identify the final implant 
orientation. The CCD angle was determined by drawing a line from the piriformis fossa down the longitudinal axis of the femur, and then measuring the angle to a line through the axis of the implant (Figure 40).

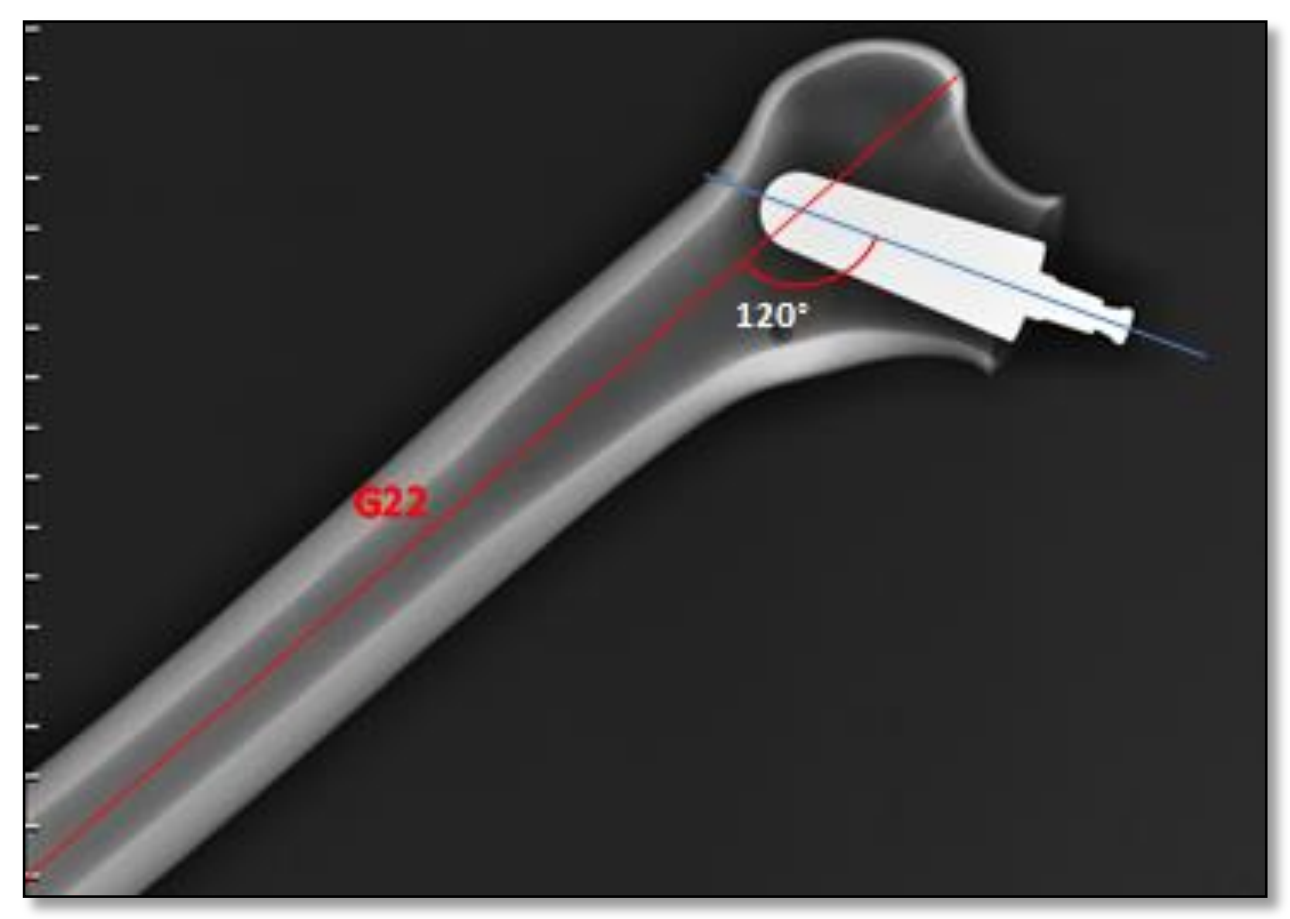

Figure 40: A/P view of a neutrally implanted femur, indicating the neutral $120^{\circ} \mathrm{CCD}$ angle formed by the long axis of the femur and the implant

The lateral angle of the femur was measured using the computer-assisted templating software EndoMap (Siemens AG, Medical Solutions, Erlangen, Germany). This software allows the user to load DICOM radiographs into the program, following which the images can be scaled, manipulated, and measured. A modified version of the Resubal method of measuring the stemneck angle (SNA) was employed in this study. ${ }^{77}$ While the Resubal method uses two lines tangent to the anterior and posterior cortical bone of the neck to determine a neutral axis, this modified method involves drawing two circles that pass through points along the cortical bone corresponding to the most proximal and distal regions of the neck. A line is then drawn between the centre points of each circle, which corresponds to the neutral axis of the neck. ${ }^{78}$ The angle between this axis and the implanted stem axis is defined as the SNA, or the lateral angle (Figure 41). 

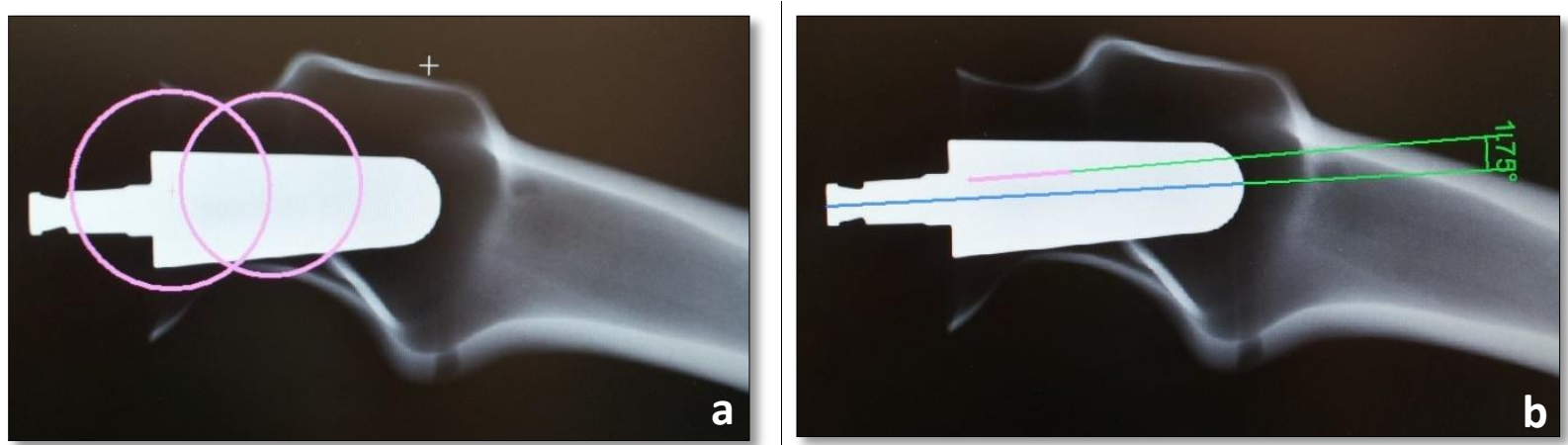

Figure 41: Lateral view of a neutrally implanted femur, depicting the modified Resubal method for determination of implant neutrality: a) tangent circles drawn on femoral neck; b) line drawn through circle centre-points, and distance to axis of implant measured

Implant positioning within the femoral neck was also determined using the $\mathrm{A} / \mathrm{P}$ and lateral radiographs of the femurs. In the A/P x-rays, a line located centrally within the isthmus of the neck was drawn at a CCD angle of $120^{\circ}$. The vertical distance from this line to the implant axis was then measured. Similarly, for the lateral radiographs, a neutral line was drawn centrally within the isthmus of the femoral neck and the perpendicular distance to the axis of the implant was determined.

\subsubsection{Femur Preparation}

\subsubsection{Potting}

In order to ensure a stable, fixed base for testing, the femurs were potted in cement-filled steel cubes measuring $88 \mathrm{~mm} \times 88 \mathrm{~mm} \times 75 \mathrm{~mm}$. Due to both machine constraints and to allow angling of the femur within the cube, the distal condyles of the femur were removed. Initially, lines were drawn along the base of the femurs at a level 60mm up from the base of the condyles (as determined by locating the condyles against a flat surface). A mitre saw was then used to remove the condyles at this preset resection level, along with the previously discussed proximal femoral head resection level.

Duct tape was placed along the base of the steel cubes to provide an enclosed box, and the femoral shafts were oriented vertically and held in place using a multiaxial clamping system. Each femur was angled at $7^{\circ}$ adduction within the coronal plane, and $0^{\circ}$ of flexion/extension in the sagittal plane in order to simulate single-legged stance. ${ }^{79-82}$ A magnetic angle level was used to ensure accurate positioning in each plane (Figure 42). A cement mixture made using commercially available anchoring cement (Flow-stone, King Packaged Materials Company, 
Burlington, ON, Canada) was then poured into each steel cube up to the brim and allowed to solidify for 24 hours, fixing the distal portion of each femur.

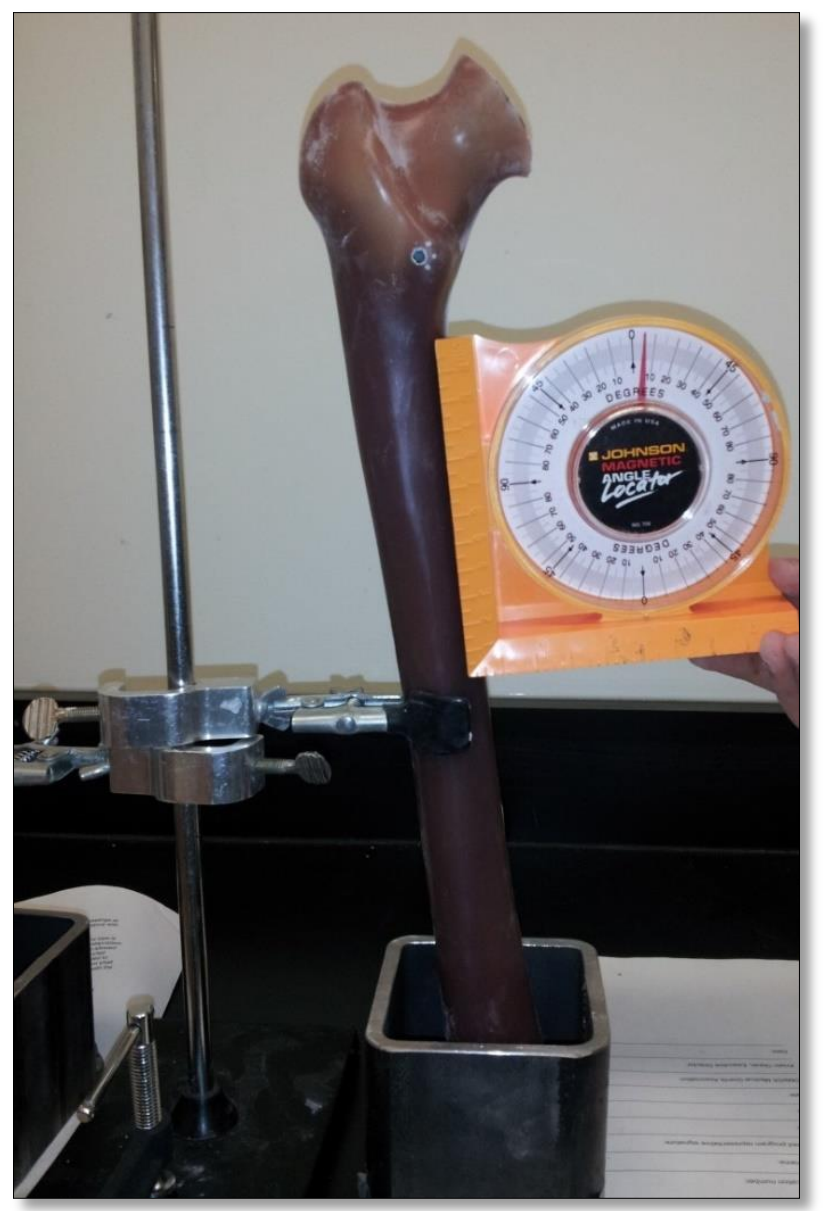

Figure 42: Synthetic femur located in a steel cube in preparation for potting, indicating the desired $7^{\circ}$ of adduction

\subsubsection{Strain Gauge Mounting}

All of the specimens were instrumented with five $350 \Omega$ uniaxial linear strain gauges (Model CEA-06-125UW-350, Vishay Measurements Group, Raleigh, NC, USA) and one $350 \Omega$ rectangular rosette gauge (Model CEA-125UY-350, Vishay Measurements Group, Raleigh, NC, USA). On the medial femoral shaft, 2 linear gauges were located on the femur surface at $234 \mathrm{~mm}$ and $189 \mathrm{~mm}$ above the top of the cement potting cube, while the lateral shaft had 3 linear gauges placed at $234 \mathrm{~mm}, 189 \mathrm{~mm}$ and $144 \mathrm{~mm}$ above the steel cube. The rosette gage was attached to the anterior side of the femur at $280 \mathrm{~mm}$ above the steel cube in order to measure the multidimensional strains of this area (Figure 43). Notwithstanding the practical problems of 
proper gauge placement on the highly complex geometry of the proximal femur, experimental gauge tests using a similar number and location of gauges were found to validate the FE model in numerous prior studies. ${ }^{83-86}$

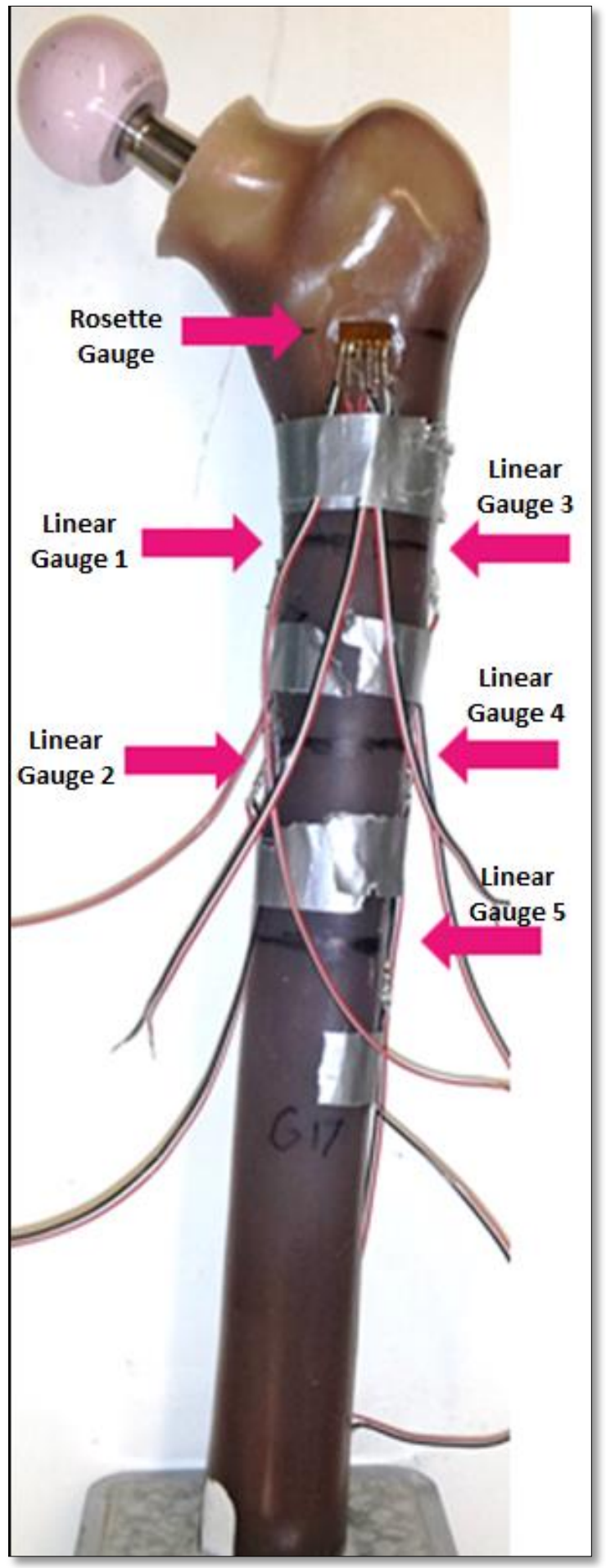

Figure 43: Gauged femur, indicating the location of the medial linear gauges (1 and 2), lateral linear gauges $(3,4$, and 5$)$, and the rosette gauge 
To ensure a good interface between the gauge and the femur, the chosen location for each gauge was initially abraded using sandpaper, and then cleaned with the manufacturer provided cleaning solution and conditioner. ${ }^{87}$ Each linear gauge was aligned such that its long axis was parallel to the longitudinal axis of the femoral shaft, and the rosette gauge was placed along a line perpendicular to the femoral shaft. An adhesive was used to affix each gauge to the femur. Wire leads were then soldered to the solder tab areas of each gauge, and secured to the femur using duct tape.

\subsubsection{Biomechanical Testing}

The direction and magnitude of loading affects stress development in the proximal femur and hence must be considered when determining the optimal stress distribution. ${ }^{8}$ It was therefore concluded that the 3 neutrally implanted femurs would undergo testing at hip angles corresponding to the maximum bounds of the gait cycle, providing repeatability measurements. Due to Instron machine size restrictions, $15^{\circ}$ of sagittal plane hip flexion was used to simulate the heel strike condition, $15^{\circ}$ of hip extension was used for the toe-off condition, and $0^{\circ}$ was used for the full single-leg stance phase. All of these values fall within or near the range limits for a typical gait cycle. ${ }^{80,81,89}$

Initially, implanted femurs were rigidly mounted at their distal potted end into an Instron 8874 tester (Instron, Norwood, MA, USA) using an industrial vice. For axial mode testing, the load cell had a $\pm 25 \mathrm{kN}$ capacity, $0.1 \mathrm{~N}$ resolution, and $\pm 0.5 \%$ accuracy. All of the femurs were loaded axially in neutral (flexion=extension $=0^{\circ}$ ) $7^{\circ}$ adduction single-leg stance. During testing of the 3 neutral femurs, the vice was manipulated into an angle of $15^{\circ}$, to allow for the flexion and extension conditions. The soldered leads connected to each strain gauge were attached to an 8channel Cronos-PL data acquisition system (IMC Mess-Systeme GmbH, Berlin, Germany) using crocodile clips (Figure 44). This system was networked with a computer for storage and data analysis. 


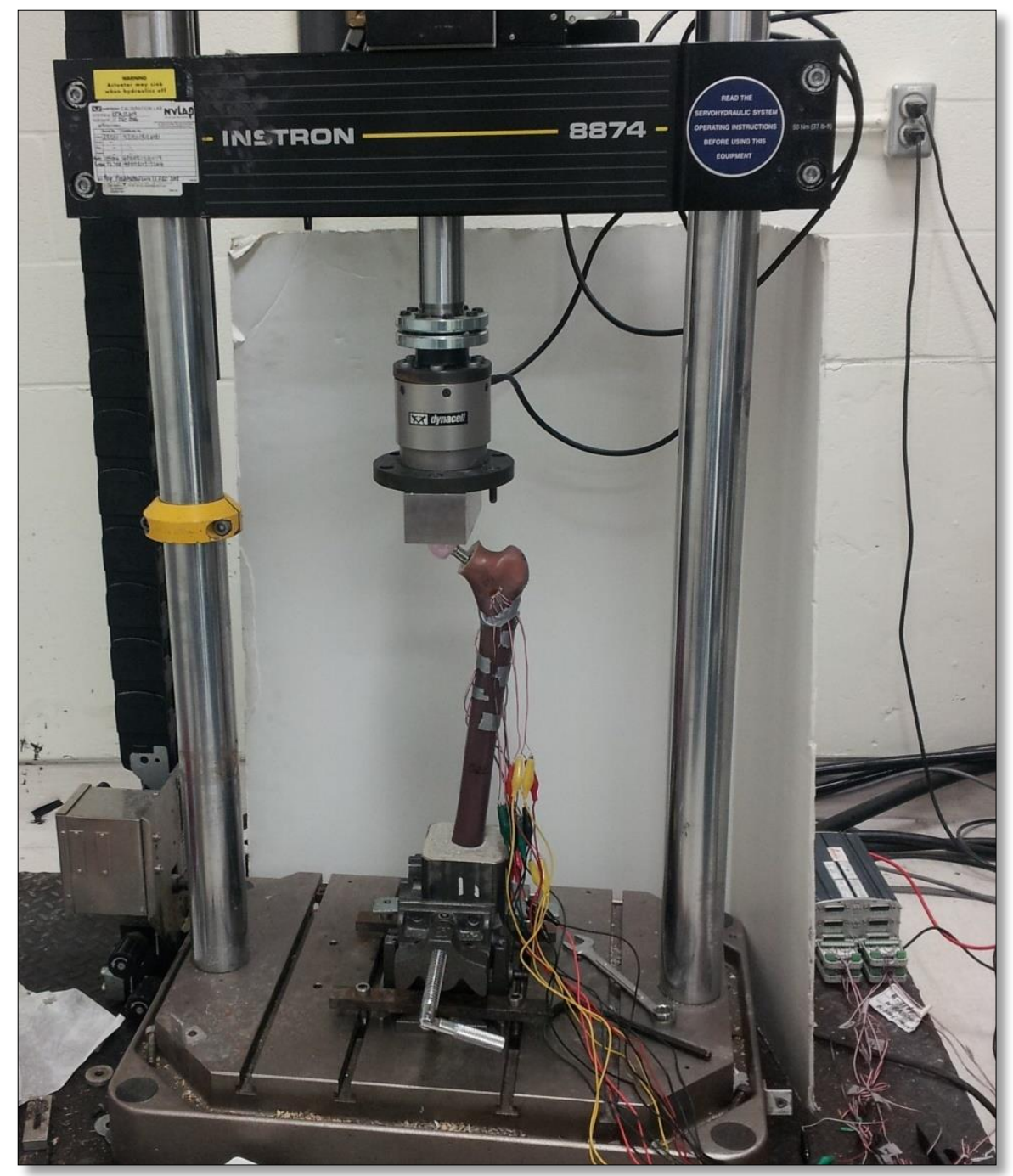

Figure 44: Experimental set-up, depicting the femur locked into a neutral orientation within the Instron machine and attached to the Cronos data acquisition system (DAS)

For load application, a 3 block load control program was developed using WaveMaker Editor Software. The first block was set to load the femur to $1000 \mathrm{~N}$ of compression in 10 seconds, the second block held the load at this value for 90 seconds, and the third block unloaded the femur in 8 seconds and then held for 2 seconds to allow the femur to completely unload. Load control was used to ensure each femur experienced a consistent load level, allowing direct comparison of strain gauge readings to the FE model. It was determined that a subclinical load of $1000 \mathrm{~N}$ would be applied, as this guaranteed that the femurs would remain in their linear elastic range and so would not undergo any permanent deformation or damage. ${ }^{83}$ Furthermore, the quasistatic loading scenario engendered very low strain rates, thereby mitigating the potential for fracture. Vaseline 
was applied to the femoral head to decrease any friction effects at the point of load application. Loads were then applied to the apex of the femoral head using a flat stainless steel plate to allow free movement of the head and avoid generation of any unanticipated lateral loads. ${ }^{90-92}$

At the start of testing, femurs were subjected to a preload of $50 \mathrm{~N}$ to minimize any mechanical slack. They were then tested using the previously defined load program. The raw data files obtained from the data acquisition system during the central 30 seconds of testing were determined to be the most stable, and hence were inputted into FAMOS V5.0 software (IMC Mess-Systeme $\mathrm{GmbH}$, Berlin, Germany) to generate corresponding average strain values over this period. Each implant orientation and the intact femur were tested 3 times consecutively to give an average of the strains developed at each strain gauge location. The 3 neutrally implanted femurs were additionally tested under extension and flexion conditions. Stiffness values were determined for each loading condition by calculating the slope of the resultant forcedisplacement graph. Such mechanical testing protocols have been used previously in a number of similar studies with good outcomes. ${ }^{65,83-86,90,93}$

\subsubsection{Strain Gauge Calculations}

The means of the strains generated at each gauge location over the course of the 3 trials were determined for every implant orientation and the intact femur. The relative standard deviation (RSD) for each average value was below 10\%, indicating a low degree of disparity. For ease of comparison to the FE model, average rosette gauge strains were first converted into principal strains (Equation 1), following which they were transformed into von Mises strains (Equation 2). The von Mises strain value for each femur was directly compared to the equivalent strain value at the rosette gauge location in the FE model, while the average linear strain results were compared to normal strains at corresponding locations in each respective FE model. Von Mises strains were used to allow for a single comparison measure, as has been done previously with von Mises stresses, increasing the consistency of manually selecting the strain gauge location within the FE model. ${ }^{94}$

Equation 1: Calculation of the principal strains $\left(\varepsilon_{1}\right.$ and $\left.\varepsilon_{2}\right)$, where $\varepsilon_{A}$, $\varepsilon_{B}$ and $\varepsilon_{C}$ correspond to strains from sections 1,2 and 3 of the rosette gauge

$$
\varepsilon_{1,2}=\frac{\varepsilon_{A}+\varepsilon_{C}}{2} \pm \frac{1}{\sqrt{2}} \sqrt{\left(\varepsilon_{A}-\varepsilon_{B}\right)^{2}+\left(\varepsilon_{B}-\varepsilon_{C}\right)^{2}}
$$


Equation 2: Calculation of the von Mises strain $\left(\varepsilon_{\mathrm{VM}}\right)$, where $\varepsilon_{1}$ and $\varepsilon_{2}$ correspond to the previously calculated principal strains

$$
\varepsilon_{V M}=\frac{2}{3} \sqrt{\varepsilon_{1}^{2}-\varepsilon_{1} \varepsilon_{2}+\varepsilon_{2}^{2}}
$$

\subsection{Finite Element Analysis}

The finite element method (FEM) is a discretization technique used for structural mechanics. It functions by subdividing a mathematical model of an object into disjointed units called elements, the geometry and degrees of freedom (DOF) for which are defined by their nodes. Each element contains its own set of discrete response equations depending on its local properties, such as imposed constraints. The overall mathematical response to a stimulus is then determined through approximation of the entirety of its finite elements. ${ }^{95}$ This is akin to the idea of approximating a circle by connecting many small straight lines. The overall precision of a model is governed by the fineness, or number of elements/nodes, within its mesh. A finer mesh will generate more accurate modelling solutions; however, it also requires more computer processing power and takes longer to solve. ${ }^{96}$

\subsubsection{Generation of Solid Models}

The first step in FE modelling is development of the geometric models. This can most easily be accomplished using 3D computer-aided design (CAD) software, such as SolidWorks. For this study, SolidWorks Standard 2014 CAD Software (SolidWorks Corp., Dassault Systemes, Concord, Waltham, MA, USA) was utilized.

\subsubsection{CAD Model of the Implant}

Due to the symmetric nature of the Silent Hip, 3D laser scanning was not required for development of the implant geometry. It was instead possible to reliably recreate the stem in SolidWorks by drawing a lengthwise, dimensioned cross-section of half of the stem, and then using the revolve feature to make the model 3D. A series of chamfers and fillets were also applied to the stem neck region to ensure that the geometry precisely matched that seen in the Silent Hip. The femoral head used in this study was similarly constructed, involving first a sketch of a dimensioned half cross-section, followed by use of the revolve feature. Cut-revolve was used to remove the centre of the head and allow for alignment with the neck of the Silent stem. Finally, a chamfer was used to attain the graded edge seen in the actual femoral head component. 
In order to allow for eventual Boolean subtractions in the FE model, a body was added to the tip of the stem to represent the $3 \mathrm{~mm}$ distal gap, while another was added to the neck of the stem to ensure the simulated surgical entry point was completely cleared of cancellous bone (Figure 45).

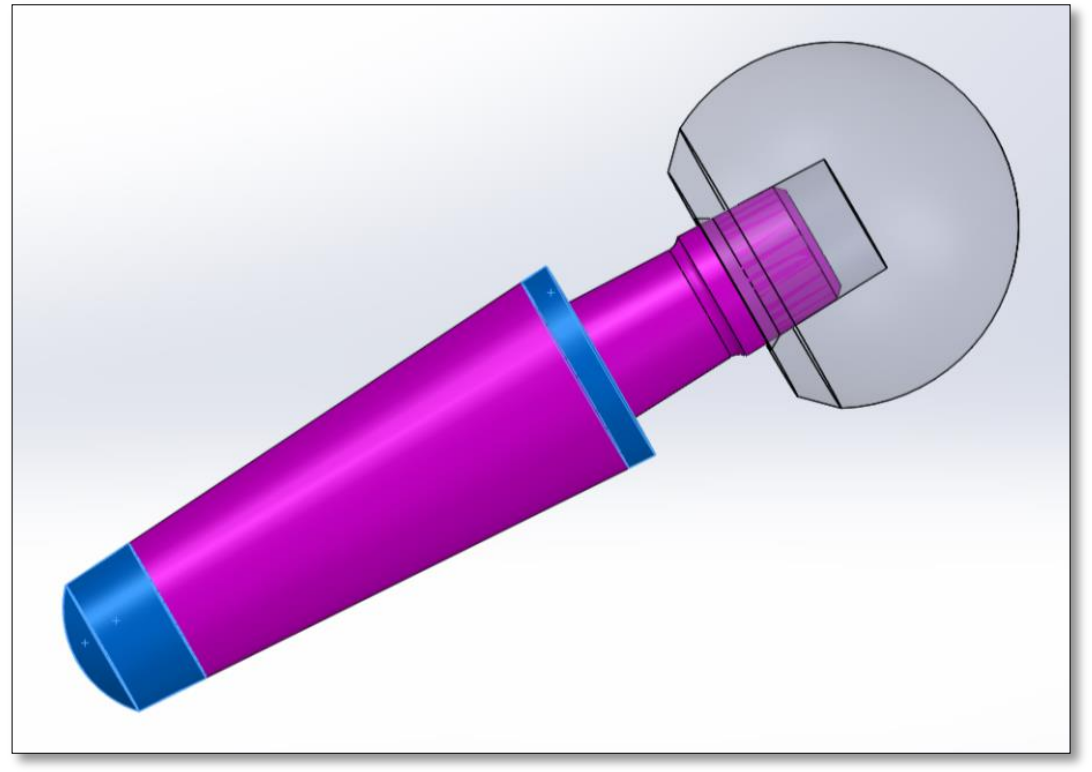

Figure 45: Silent Hip model fitted to the femoral head, indicating location of extra bodies in blue for eventual Boolean operations in ANSYS

\subsubsection{CAD Model of the Synthetic Femur}

The femoral model used for this study was a previously validated computed tomography (CT)scanned model of the left, $4^{\text {th }}$ generation synthetic femur. ${ }^{93}$ This model was created by obtaining cross-sectional slices of the femur at every $0.5 \mathrm{~mm}$ along its length, stocking the images in the DICOM format, and then importing them into Mimics ${ }^{\circledR}$ Medical Imaging Software (The Materialise Group, Leuven, Belgium) to allow for 3D model generation. ${ }^{83,93,97}$ Surfaces corresponding to both cancellous and cortical bone boundaries were obtained and hence the femur model consisted of both cancellous and cortical bone bodies. This model was imported into SolidWorks for assembly with the Silent stem and head models.

\subsubsection{SolidWorks Assembly}

Once all of the models had been assembled in SolidWorks, additional modifications were completed to match the compiled model to experimental conditions. Firstly, it was determined that the CT scan based femur model had no intramedullary canal, thus one was created by using the cut-extrude feature to remove a dimensioned portion of the cancellous bone. The cut-extrude feature was further used to remove the head and condyles of the femur at levels corresponding to 
the experimental resection lines. A drawing was also projected onto the base of the femur at the height and angle of the cement cube to permit fixation of these faces during FE modelling. The stem and head were aligned and mated along their axes, then positioned neutrally within the proximal femur to simulate the neutral implant orientation. Finally, a rectangle replicating the flat loading plate was generated and placed proximal to the femur for eventual simulations of experimental loading conditions (Figure 46). The completed assembly was subsequently imported into the FEA software.

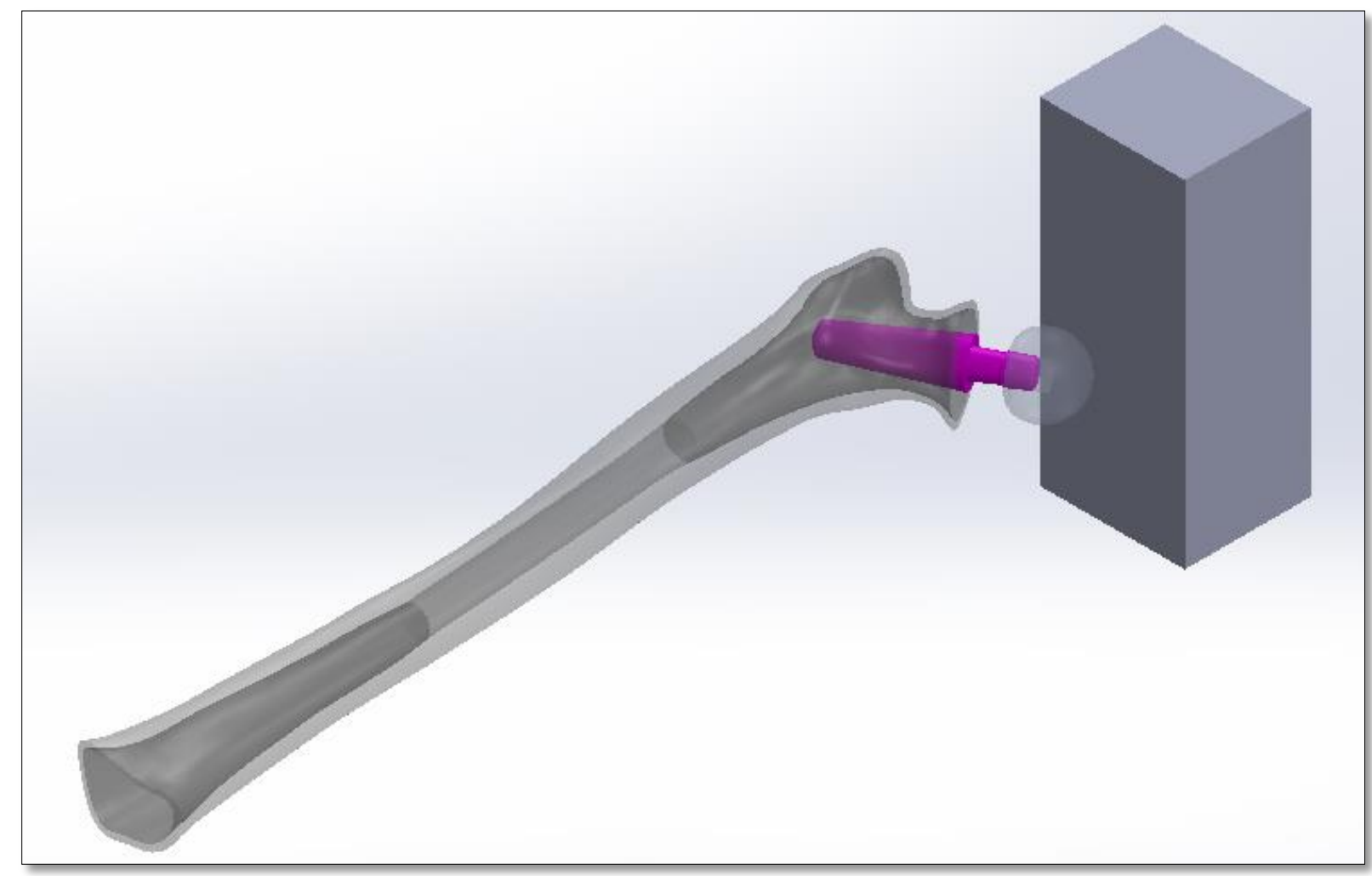

Figure 46: CAD model of the implanted femur assembly with the Silent stem in neutral

\subsubsection{ANSYS Workbench for Finite Element Analysis}

The software used for FEA in this study was ANSYS Workbench 15.0 (ANSYS Inc., Canonsburg, PA, USA). In general, static structural FE models were made to imitate each implant orientation for validation purposes. A number of additional models were then created to reproduce the experimental neutral, flexion, and extension conditions. These were subsequently modified to meet the run requirements specified in the DOE, allowing for individual DOE assessment of each. 


\subsubsection{ANSYS Engineering Data}

Material properties can be set within the Engineering Data tab in ANSYS Workbench. This tab allows you to add materials and then define their properties, for use throughout the rest of the models. In order for ANSYS to solve linear static structural analyses, it requires the Young's modulus $(E)$ and Poisson's ratio $(v)$ for each material. The values used in this study were the previously determined material properties for each material (Table 5).

Table 5: List of the Young's modulus and Poisson's ratio used for each material in the ANSYS Workbench simulations

\begin{tabular}{|c|c|c|c|}
\hline Material & Loading Mode & Young's Modulus (GPa) & Poisson's Ratio \\
\hline Cortical Bone & Longitudinal Compression & 16.7 & 0.26 \\
\hline Cancellous Bone & Compression & 0.155 & 0.3 \\
\hline $\begin{array}{l}\text { BIOLOX }{ }^{\circledR} \text { delta ceramic } \\
\text { (Femoral head) }\end{array}$ & N/A & 358 & 0.24 \\
\hline $\begin{array}{c}\text { Titanium alloy (Ti-6Al-4V) } \\
\text { (Silent stem) }\end{array}$ & N/A & 110 & 0.31 \\
\hline
\end{tabular}

As discussed in Section 2.1.2, bone is an inhomogeneous anisotropic material that is stronger in compression than in tension. While it is possible to assign inhomogeneous anisotropic properties to bone in an FE model, sawbones are homogeneous and transversely isotropic, and thus are better represented by assuming homogeneous linearly elastic continuum properties with isotropic

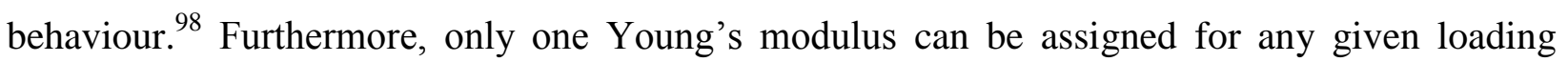
direction, and thus the Young's moduli corresponding to the compression condition was selected for this model as it was assumed to be the most likely loading state. These assumptions are commonly used when modelling synthetic bone, with good results. ${ }^{79,93,99,100}$ The inherent viscoelasticity of bone was also not included in the FE model as the static loading condition produced a strain rate so low that it had a negligible effect upon the bone stiffness.

\subsubsection{ANSYS DesignModeler}

The model generated in SolidWorks was saved as a STEP file, which is the ISO standard for representing CAD models and allows for dissociation of the ANSYS imported file from the original modifiable file. The saved CAD model was then imported into ANSYS DesignModeler, and the global coordinate system was defined to be consistent with the original SolidWorks model. 
ANSYS DesignModeler utilizes a tree outline with each successive geometry modification listed in order of its activation (Figure 47). For this study, an axis was first defined along the shaft of the femur, to enable evaluation of the longitudinal strains. Body transformations corresponding to each of the potential orientations were then generated. Three translational transformations were defined along planes corresponding to the posterior, anterior and inferior positions. Three rotational transformations were then defined using components to describe the valgus, varus and anteversion angles. In each orientation definition, it was ensured that the stem protruded from the proximal femoral resection by no more than $3 \mathrm{~mm}$.

Boolean subtractions were used to remove any cancellous and cortical bone that overlapped with the stem. At this point, the extra bodies placed at the top and the tip of the stem were also subtracted to create a $3 \mathrm{~mm}$ distal gap, and to remove any proximal cancellous bone that may have overlapped with the stem when its angulation caused recession.

Finally, the direction of loading was simulated by maneuvering the flat loading plate into a position representative of $7^{\circ}$ of femoral adduction, and neutral sagittal plane angulation (except for those models verifying the experimental flexion or extension conditions). The plate was then translated to a position just above the apex of the femoral head. At this time, a small circle with a diameter of $3 \mathrm{~mm}$ was drawn onto the base plane of the loading plate and projected onto the head to simulate the point of load application. The local coordinate system of the plate at this location was then used to define the direction of load application. 


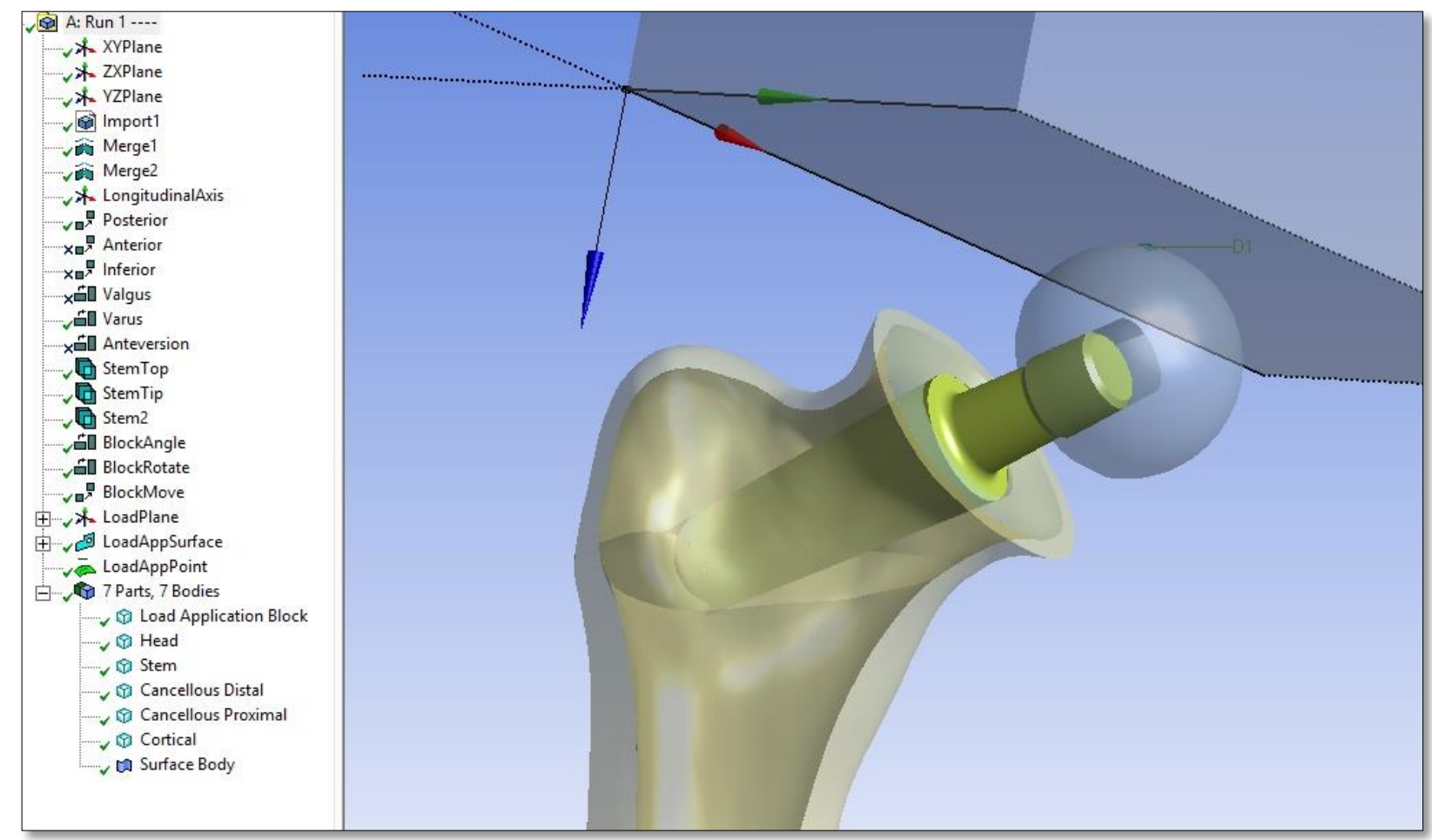

Figure 47: Screenshot of ANSYS DesignModeler, depicting the tree outline with each modification step

\subsubsection{ANSYS Mechanical}

Once the geometry had been defined, ANSYS Mechanical was used to set all of the boundary conditions and constraints, generate the mesh, and ultimately solve the model.

\section{Geometry}

Within the geometry tab, the user can define each of the bodies within the model and set their corresponding material properties. Each body was therefore assigned to its respective material as defined previously in Engineering Data. Furthermore, the load plate body was suppressed for this study, as only the load application point and its coordinate system were required for analysis.

\section{Coordinate Systems}

A number of coordinate systems were either imported from ANSYS DesignModeler or defined for meshing purposes. The longitudinal axis coordinate system and the load plane coordinate system were imported from DesignModeler, while additional coordinate systems in the neck and body of the stem were defined for eventual use in mesh generation. 


\section{Contacts}

When assemblies of solid bodies are imported, contact regions between each body are automatically assigned. Contact definition allows separate bodies to have different sized and thus non-matching meshes at their boundaries. Contact between bodies can be either linear and require only 1 iteration to solve, such as bonded and no separation contact, or nonlinear and necessitate multiple iterations, which includes frictionless, rough, and frictional contact. ${ }^{96}$ Due to their iterative nature, nonlinear contacts tend to require more processing power and take much longer to solve than linear contacts, and hence most models do not use nonlinear contact unless absolutely necessary.

For this study, it was determined that contact between the stem and the cancellous/cortical bone could be set to bonded, which would simulate the initial press-fit and long-term osseointegration that would be expected to occur. This is a common assumption when modelling the bone-implant interface. $^{79,93,101}$ Contact between the cortical and cancellous bone was also set to bonded, as these bodies were assumed to be rigidly fixed to one another in the synthetic femur. Finally, contact between the stem and the ceramic femoral head was set to bonded to simulate the $12 / 14$ taper press fit.

In experimental conditions, contact between the head and the load plate was not frictionless, despite the application of Vaseline to reduce friction. Static friction was present initially, and represented the initial force required to overcome the static cohesion of the head to the load block, following which kinetic friction was present. However, setting frictional contact within an FE model requires knowledge of the friction coefficient, and hence it is commonly initially modeled as frictionless contact. ${ }^{65}$ A model simulating frictionless contact between the load plate and the head was therefore developed for the neutral condition, with the movement of the flat loading plate restricted in all directions except for its vertical $z$-plane. Frictionless contact is nonlinear in nature, and thus frequently exhibits non-converging solutions with extended computational times. It is therefore beneficial to utilize a linear model when possible. Since the flat load plate was only able to apply force in its vertical direction and sliding was assumed to be minimal, it was therefore possible to remove the plate entirely and instead apply the load directly to the femoral head, yielding a linear loading situation. Stress results from the frictionless model were compared to the linear model, and found to differ by an average of only $6 \%$ across four Regions of Interest, as defined in the Section Named Selections (Figure 48). Stress results for a 
model defined with the most realistic frictional contact would likely fall between the nonlinear frictionless model and the linear direct load application model. Hence, given the minimal difference in the stress results between these two extremes, it can be assumed that the linear model is a good approximation for experimental conditions, and hence the direct load application approach was used in this study.
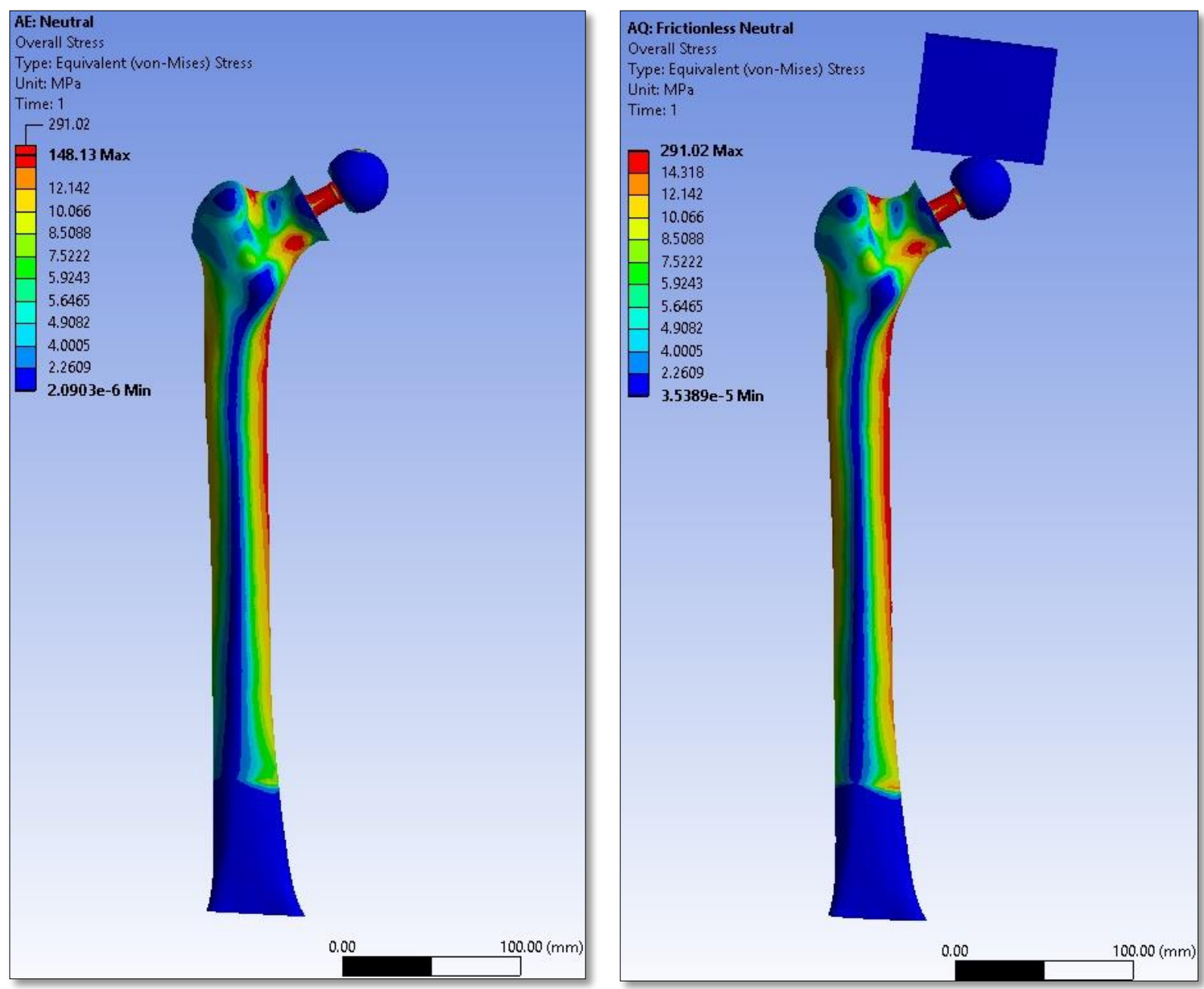

Figure 48: Comparison of linear neutral model with load applied directly to femoral head (left) versus a nonlinear neutral model with contact between head and load plate set to frictionless (right), equivalently scaled in von Mises stresses (MPa) for comparison purposes

\section{Mesh}

In FE modelling, the mesh refers to the compilation of all of the elements and nodes used to approximate the model. Selection of an appropriate mesh is an iterative process that requires selection of element types, sizes, and regions of refinement. For this study, all bodies were 
meshed using 10-node tetrahedral elements, which have quadratic displacement behaviour and 3 DOF at each node (Figure 49). ${ }^{65}$ In a previous study, these elements were shown to be preferred over mapped, hexagonal, or voxel meshes for use with the human femur. ${ }^{102}$

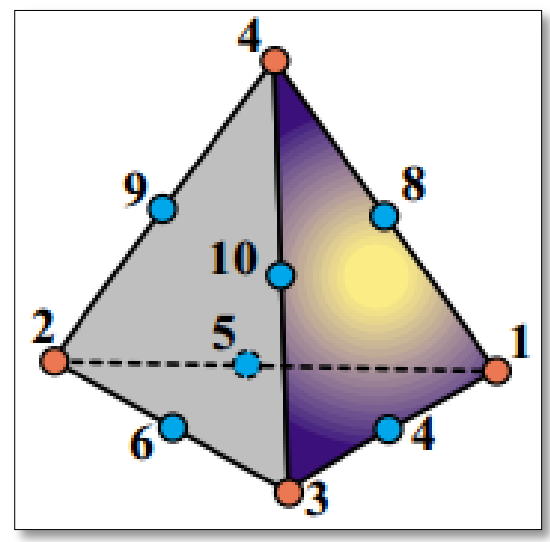

\section{Figure 49: Depiction of a 10-node quadratic tetrahedron ${ }^{95}$}

All of the element sizes used in this study were chosen based on evaluation of mesh convergence. This involved individually varying the element sizes for each method from $8 \mathrm{~mm}$ down to $2 \mathrm{~mm}$, and examining the difference in the resultant max stresses, max deformations, and numbers of elements and nodes. Furthermore, mesh sensitivity analysis was completed using the relevance slider tool, which defines either a coarse mesh (negative relevance to -100) or a fine mesh (positive relevance to +100 ). At a relevance of 80 , the modelling solution with regard to stress and deformation was found to level off, yielding values that differed by less than $1 \%$ from a mesh with a relevance of $100 .^{101}$

For the overall mesh, element size was set to $5 \mathrm{~mm}$, with a relevance of 80 . Additional methods were added to the cortical and cancellous bones to specify that they be meshed with tetrahedral elements. The proximal portion of the femur was determined to be an area of interest, and thus two spheres of influence were added to refine the element size of both the stem-head construct and the cortical and cancellous bone in this region to a size of $2.8 \mathrm{~mm}$. Individual refinements were added to the surface of the stem where it came into contact with the cancellous bone and the femoral head, and matching refinements were added to the surfaces of the head and the cancellous bone (Figure 50). This minimized the potential for mesh interferences that can arise at boundaries with material changes. ${ }^{95}$ 
Finally, two sizing features were added to the cortical and cancellous bone to make their average element size approximately 4mm. Overall, each model had an average of 178000 elements, and 270000 nodes (Figure 51).

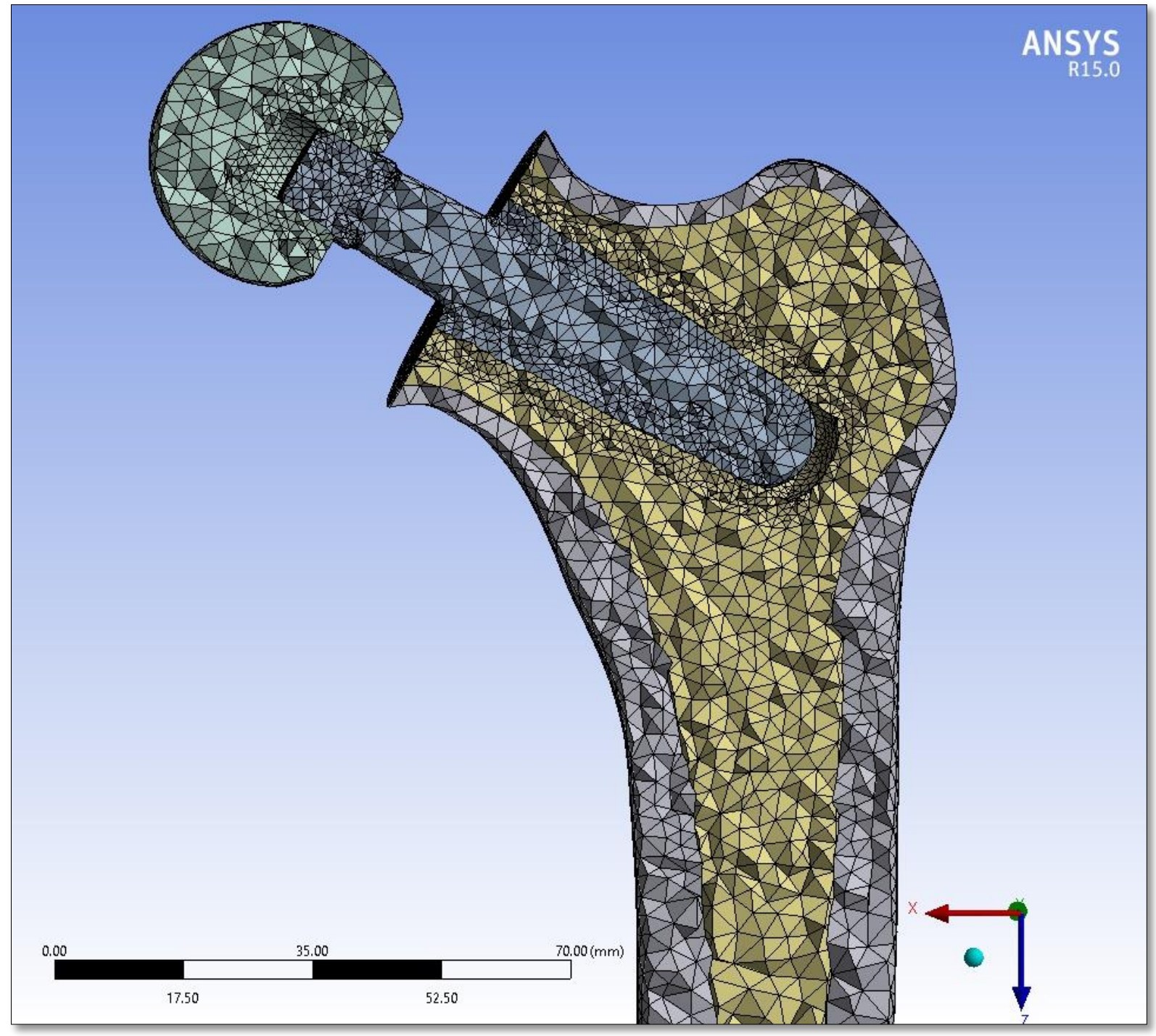

Figure 50: Proximal cross-section of the mesh used for this study, depicting the finer mesh and areas of refinement 


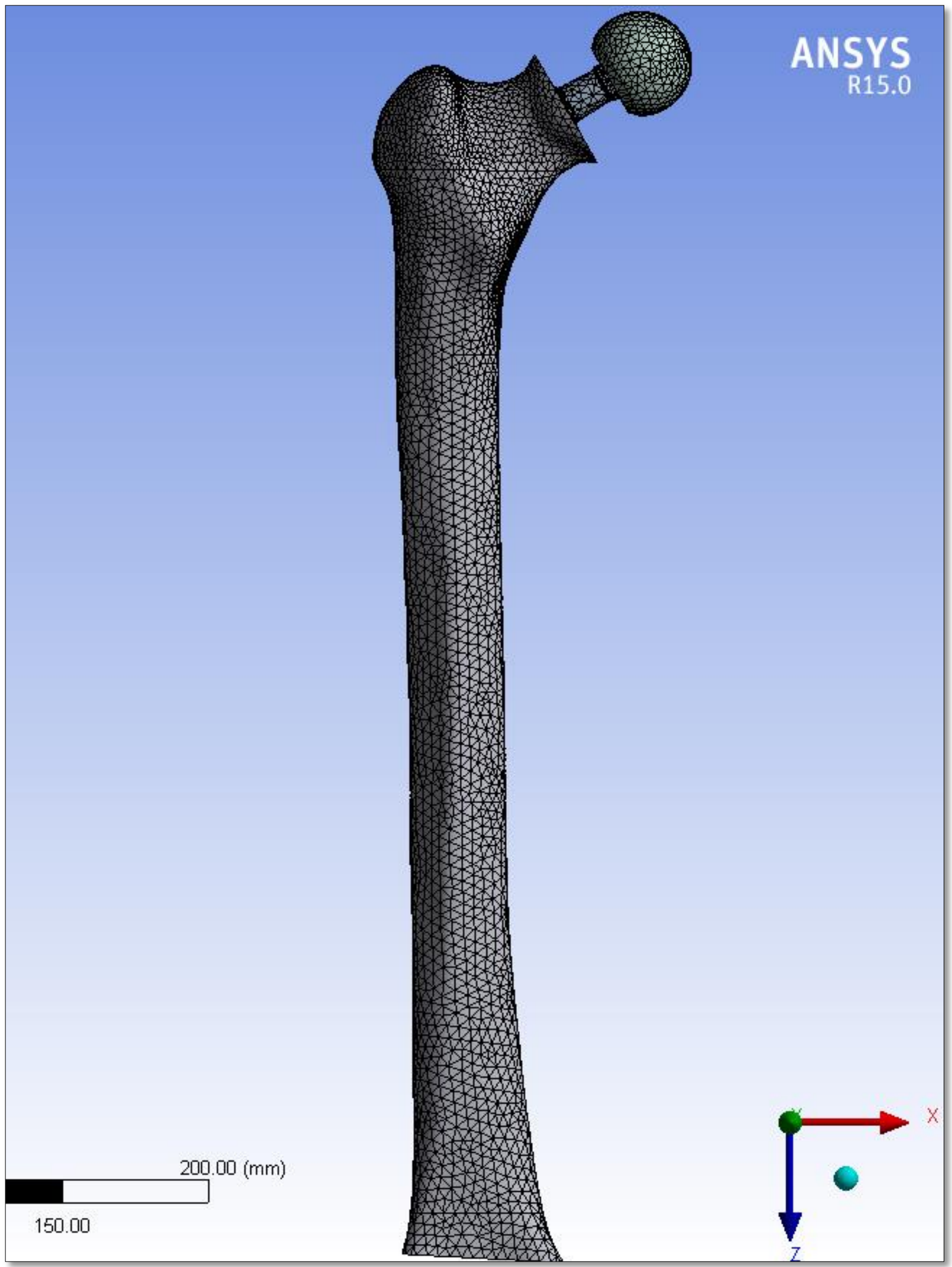

Figure 51: Screenshot of the final mesh used in this study 


\section{Named Selections}

It was determined that to best analyze the stress distribution within the proximal femur, specific Regions of Interest should be defined. These areas were based on those locations most likely to suffer catastrophic failure, such as the inferior and superior neck region. ${ }^{52,103}$ The potential for distal loading was also considered significant, and hence the region lateral to the lesser trochanter was of interest. ${ }^{61}$ These regions were addressed in the FE model by generation of named nodal selections. Nodal selection involves specifying a location-based region of interest that is then converted into its meshed nodes. It was used for this study as at it allows for consistent geometry selection between models regardless of the underlying mesh.

Given the much lower Young's modulus of cancellous bone, the stress distribution within this structure was found to be very low when compared to that of cortical bone. Furthermore, application of strain gauges can only evaluate the cortical surface strain, and cortical bone stress is a commonly used assessor of stress shielding. ${ }^{42,104-106}$ Finally, as this was a comparative study, it is likely that similar trends in the relative stress distribution would be found in the cancellous bone. Thus for the following evaluation, only the cortical bone body was considered.

Four regions of interest were described within the nodal structure of the proximal cortical bone. The first region (Proximal Region) encompassed the entire proximal region of the implanted femur to allow for overall evaluation of the stress in this area (Figure 52). The second (Inferior Neck) and third (Superior Neck) regions of interest took into account loading of the femoral neck (Figure 53 and Figure 54). Finally, the fourth region (Lateral Region) evaluated the extent of endosteal loading present in the given implant orientation (Figure 55). Each of these regions was identically recreated in the intact femur model for comparison purposes. 


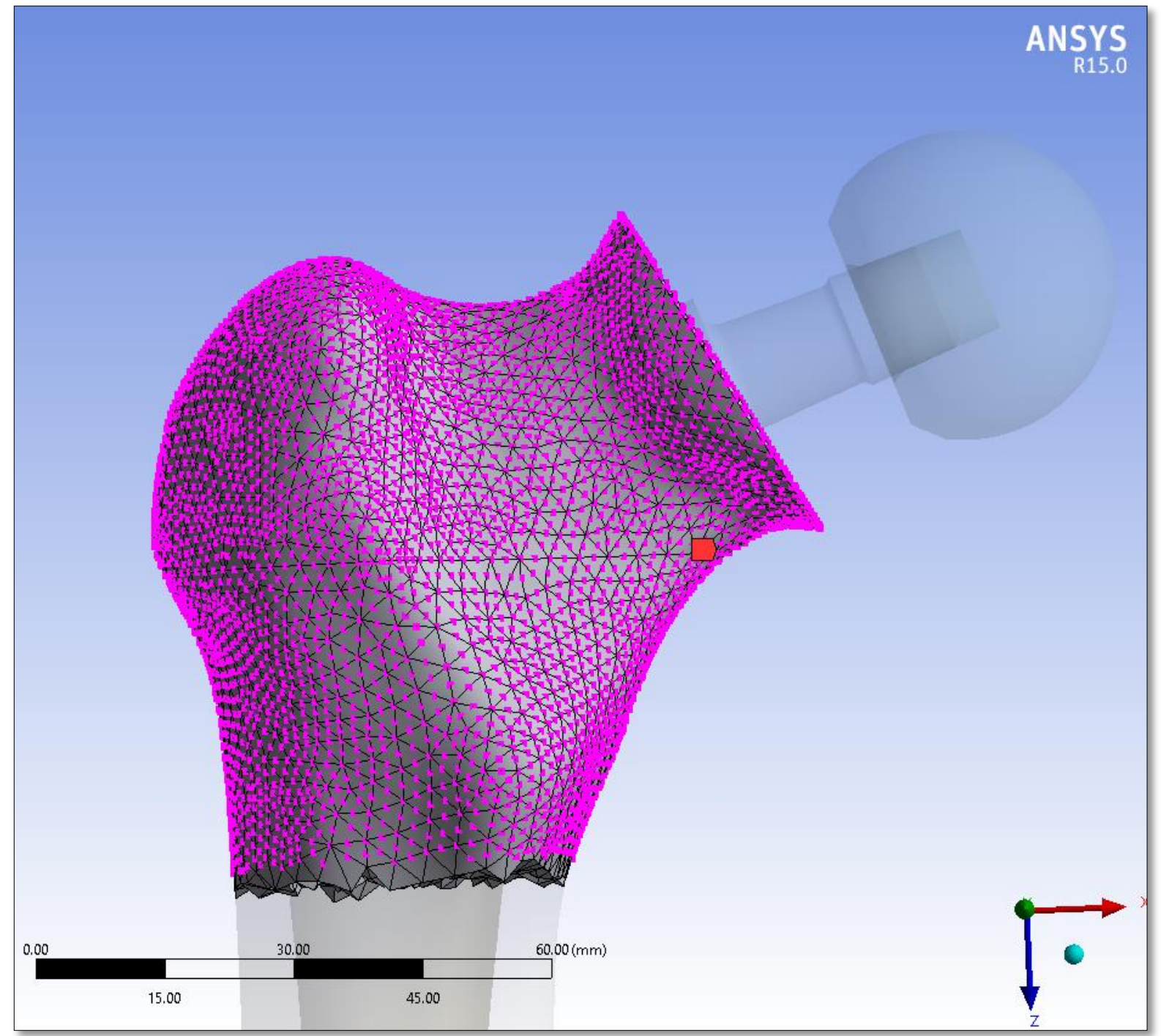

Figure 52: Proximal Region of interest selected based on a global coordinate system location, where the pink dots indicate underlying nodes 


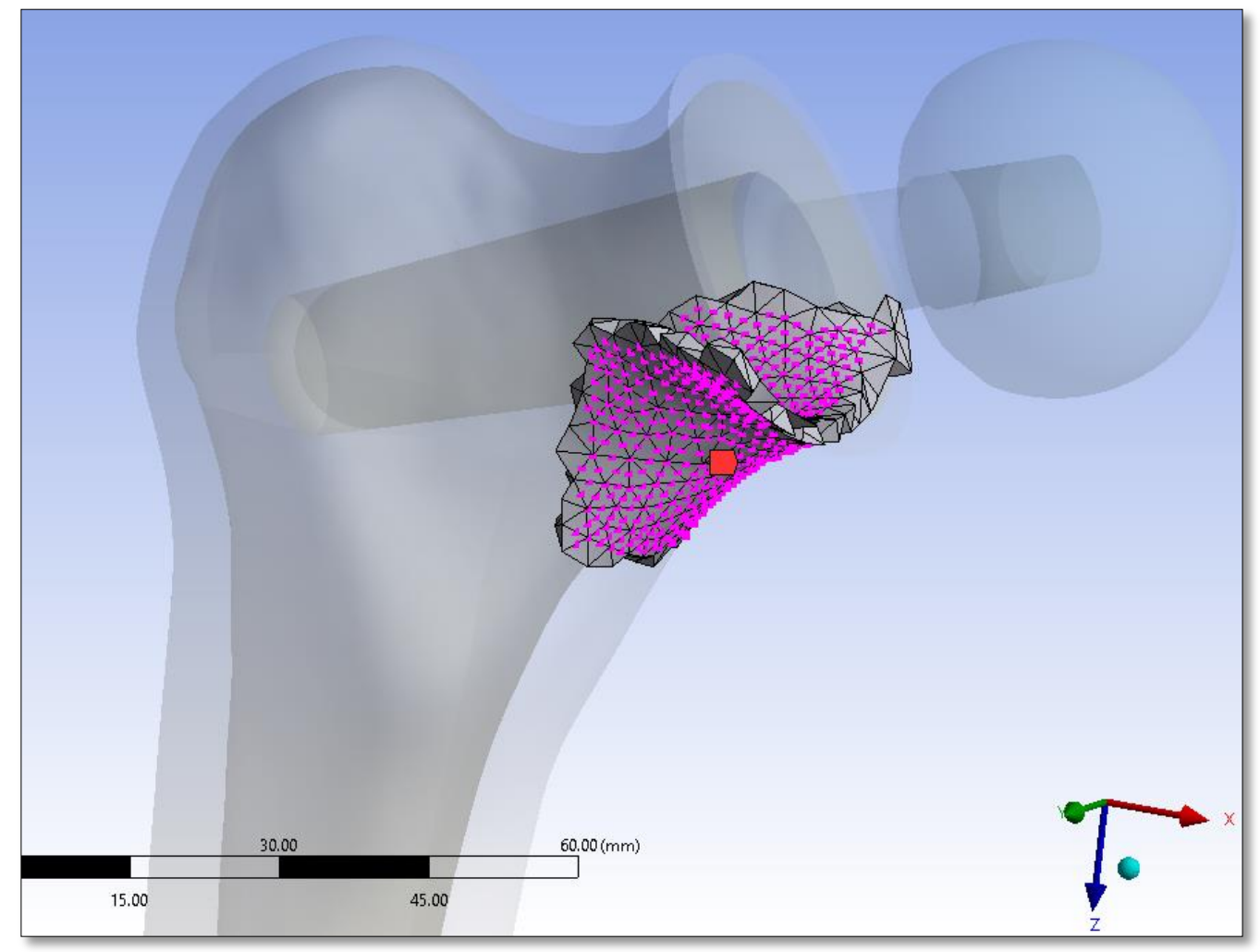

Figure 53: Inferior Neck Region, depicting selected nodes

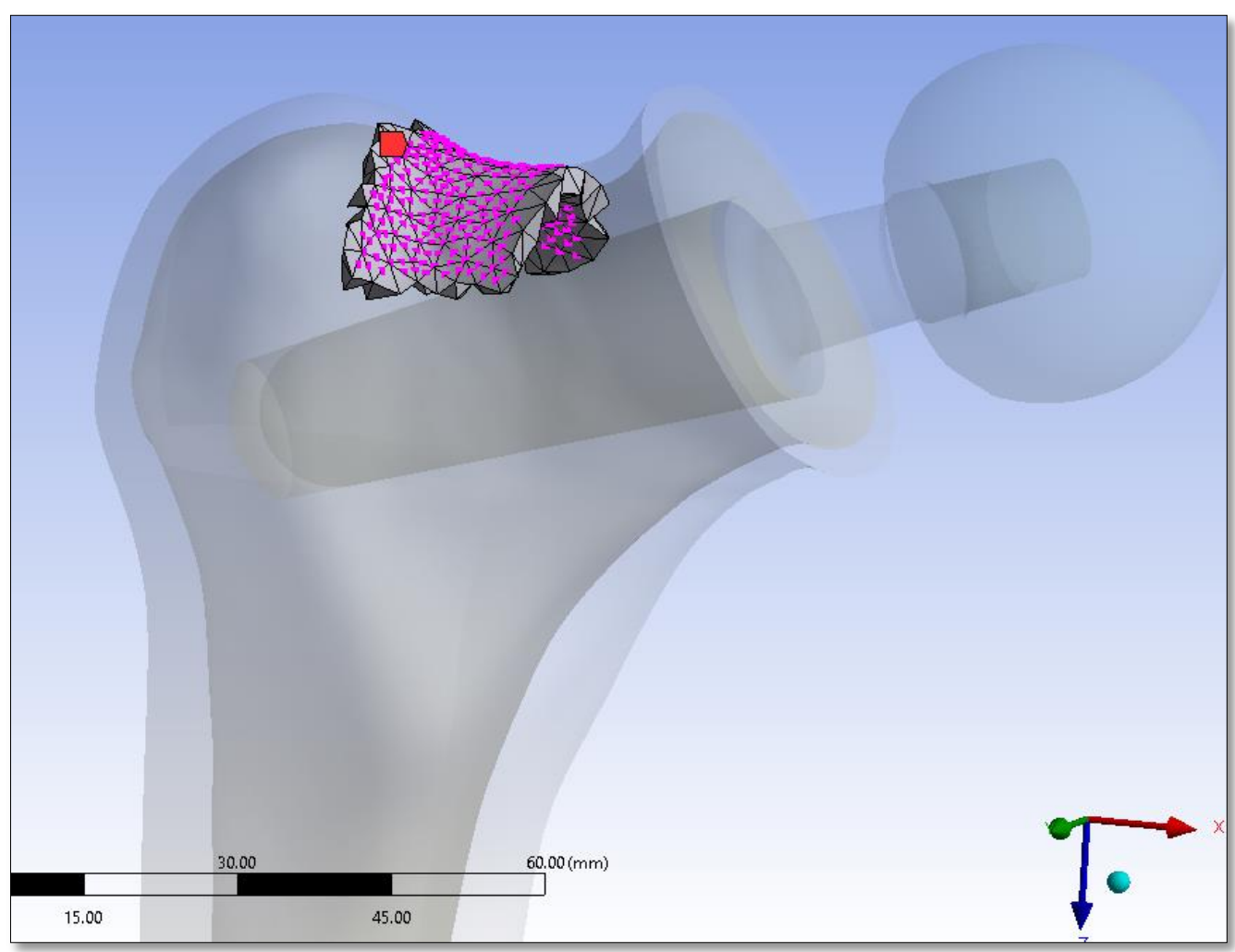

Figure 54: Superior Neck Region, depicting selected nodes 


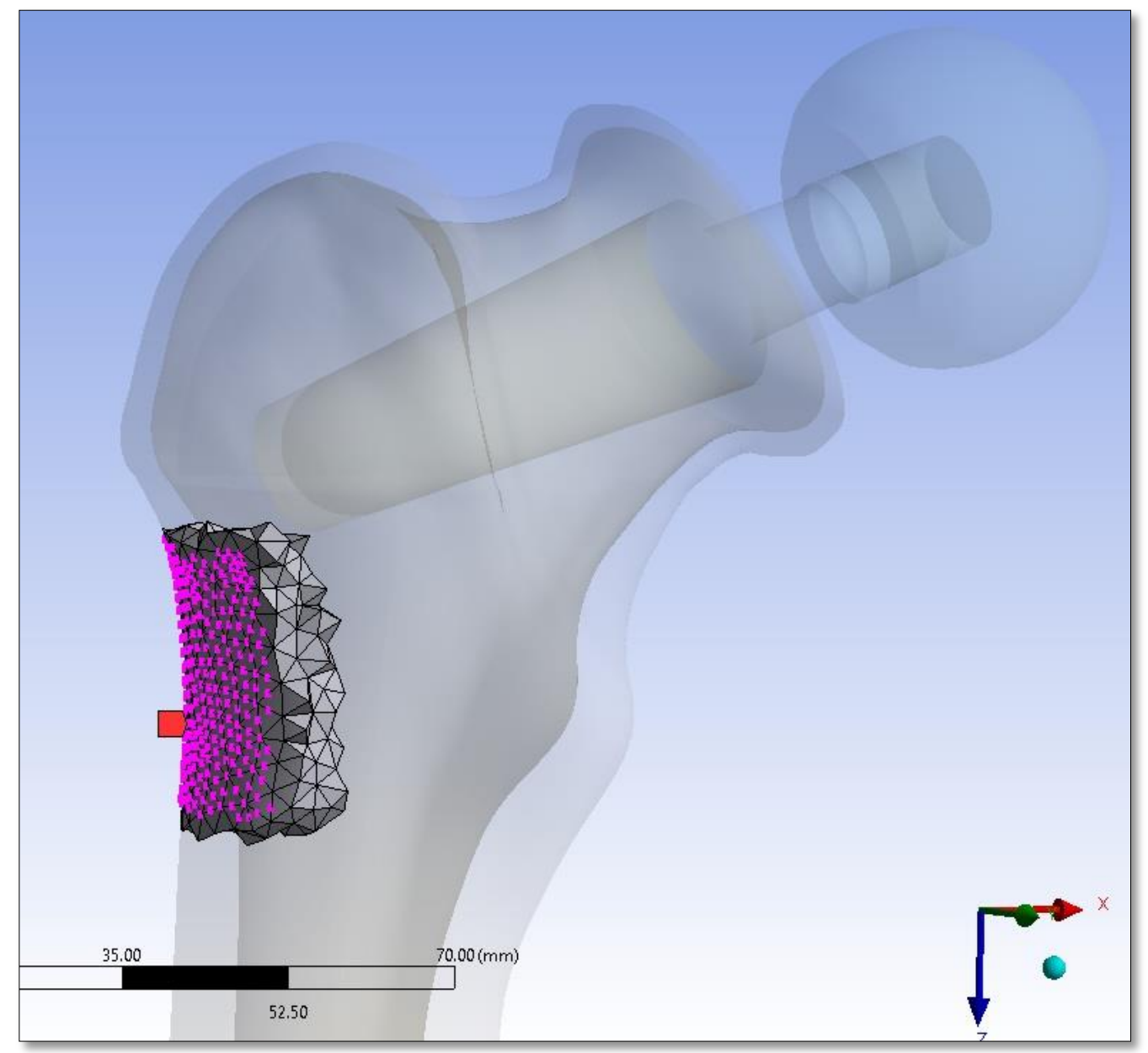

Figure 55: Lateral Region, depicting selected nodes

\section{Constraints}

The final step in generating an FE model is to select a set of constraints which govern what forces are applied and which DOF in the model are restrained. In order to replicate experimental conditions, a force of $1000 \mathrm{~N}$ was applied along the load plane coordinate system, directly onto the femoral head at the previously defined load application point. A fixed support was then applied to the distal faces of the femur corresponding to the height of the cement block, simulating the potted femur.

\section{Solution}

The solution tab allows the user to select a number of outcome measures, including von Mises stress, von Mises strain, normal strain, and deformation. Each measure can be applied to a particular body or face, or a combination of either one. For initial validation purposes in this study, the femoral von Mises strains and the normal strains along the longitudinal axis were 
generated. The von Mises stresses for each of the predefined regions of interest were also retrieved. Deflection in the z-plane at the load application point was found in order to calculate the stiffness of the model. The nodal von Mises stress results from each of the regions of interest were exported and saved into Excel files. The average nodal stress, standard deviation, and $90^{\text {th }}$ percentile stress for each region was then calculated and compiled for analysis.

\subsection{Design of Experiments}

Design of experiments (DOE) is a statistical technique employed to evaluate whether system inputs have a significant effect on resulting outputs. It can also be used to determine whether any inputs have interaction effects, and what input levels would yield an optimal output response. Experimental Designs have 3 components: factors, levels and responses, each of which are entered by the experimenter. Factors consist of the inputs to the process or system, and are usually comprised of controllable or uncontrollable variables. Levels are the settings of each factor, and are typically defined as being high or low. The responses are the desired outcome variables for the experiment, which drive the optimization of the system (Figure 56).

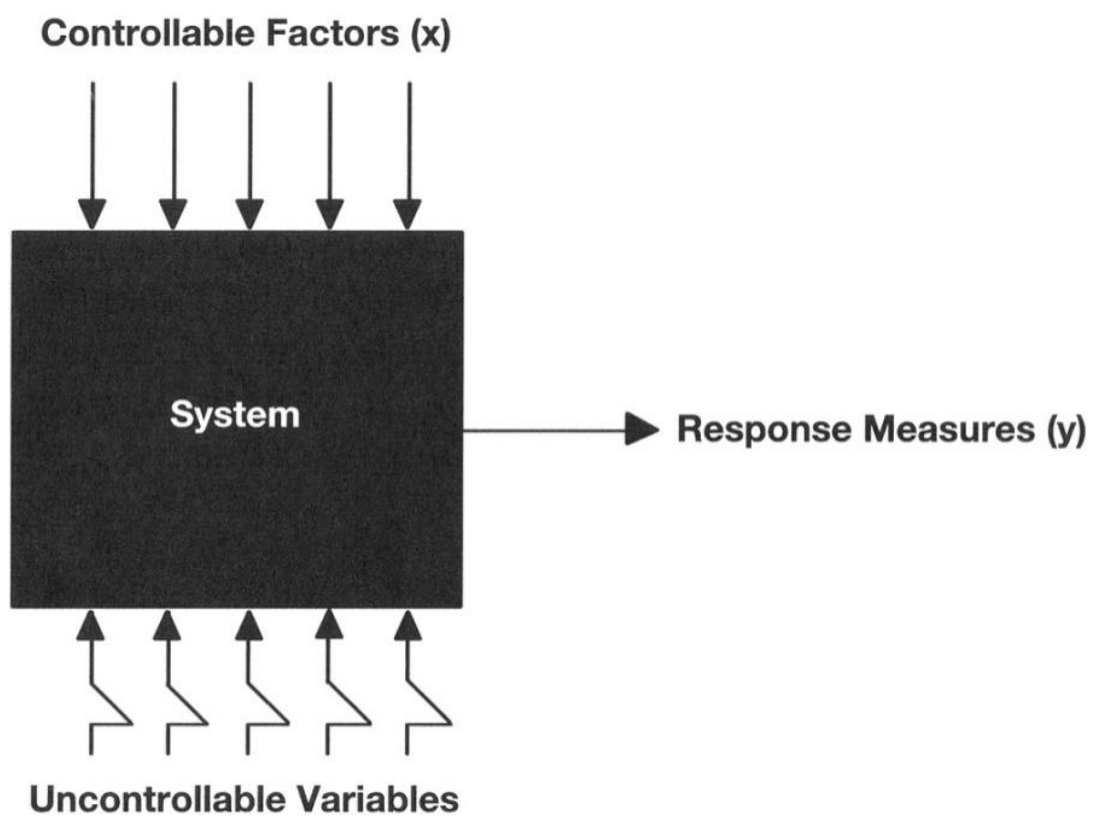

Figure 56: Depiction of a black-box system with variable inputs and corresponding responses ${ }^{107}$

In this study, there were 4 defined factors, which were given by the examined angles and positions and their corresponding maximum achievable values as determined experimentally (Table 6). 
Table 6: DOE factors and corresponding maximum levels, as achieved in the biomechanical tests

\begin{tabular}{|l|cc|}
\hline Factor & Low Level & High Level \\
\hline Varus/Valgus $\left(^{\circ}\right)$ & -10 & 10 \\
\hline Anteversion $\left(^{\circ}\right)$ & 0 & 10 \\
\hline Posterior/Anterior $(\mathbf{m m})$ & -2 & 2 \\
\hline Inferior $(\mathbf{m m})$ & 0 & 4 \\
\hline
\end{tabular}

\subsubsection{OFAT versus Factorial Design}

The simplest method of experimental design is the one-factor-at-a-time (OFAT) method, which involves varying a single factor at a time while all of the others are kept fixed. This method is not ideal for a number of reasons, chief of which is the inability of OFAT to explore interaction effects between factors. OFAT design also requires a greater number of experimental runs when compared to some factorial designs, which can be problematic when time and resources are an issue. Factorial designs address many of these concerns by allowing the user to vary numerous factors simultaneously, which also has the advantage of increasing the inductive basis from which to draw inferences about the process. For example, a two-level factorial design might consist of 3 factors varied between high and low levels. The number of experimental runs required by a two-level factorial design is $2^{\mathrm{k}}$, where $\mathrm{k}$ is the number of factors. Thus in this case, only 8 runs would be required by a factorial design, which gives the same statistical power as 16 OFAT runs (Figure 57). ${ }^{108}$
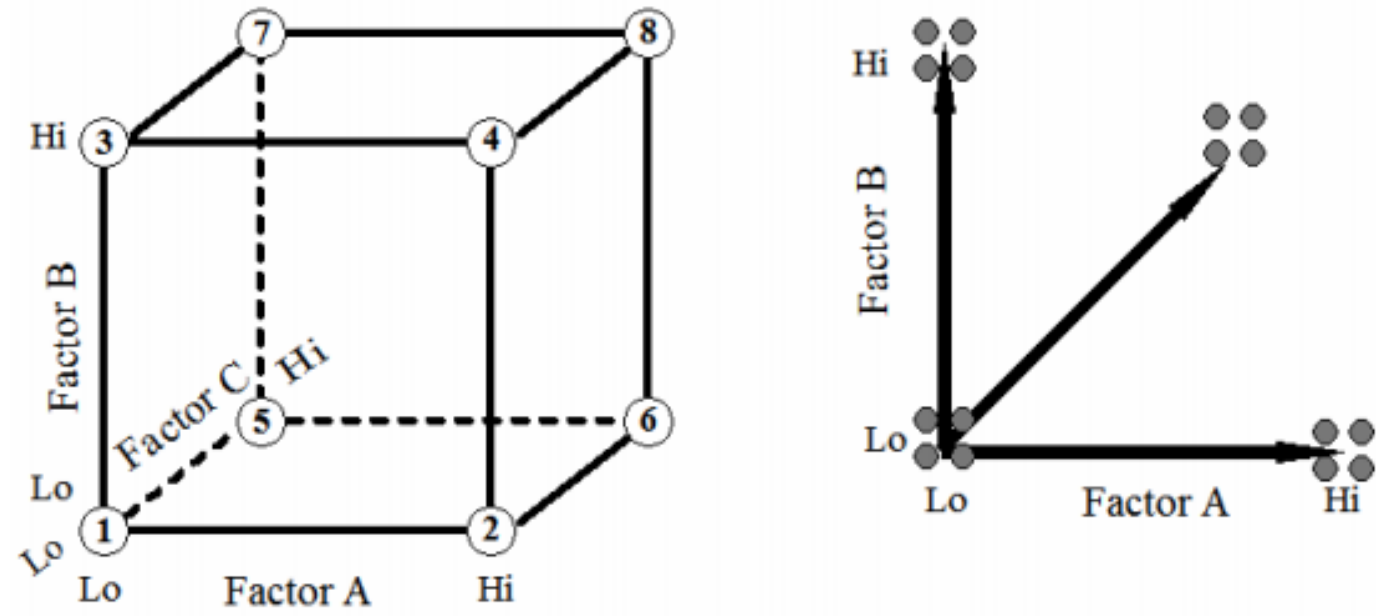

Figure 57: Two-level factorial design depicting the contents of each run, and the volume covered by the design ${ }^{108}$ 
Through the addition of centre points, which are midpoints between the high and low factor levels, it is possible for two-level factorial designs to sense the presence of nonlinearities and curvature. ${ }^{108}$ However, these designs are unable to fit a model to the given surface, and thus they must be upgraded to a more complex design.

\subsubsection{Response Surface Methodology Designs}

The goal of response surface methodologies (RSM) is to fit a 3D contour map to a response, thereby reflecting any significant curvature. ${ }^{108}$ This can be accomplished through use of either a three-level factorial design or a central composite design (CCD).

In three-level factorial designs, the number of experimental runs required is $3^{\mathrm{k}}$, where $\mathrm{k}$ is the number of input factors. ${ }^{107}$ However, depending on the amount of factors being investigated, this number can get quite large very quickly. For example, analysis of 4 factors would require 81 runs, which is an excessively large number for any given experimental budget. An alternative to this full three-level factorial is to use a CCD.

CCD differ from two-level factorial designs in that they contain axial star points in addition to centre points, which increases the number of factor combinations included in the DOE, allowing better estimation of response curvature (Figure 58). ${ }^{107}$ Furthermore, the number of experimental runs required by a 4 factor $\mathrm{CCD}$ is only 30 , which is much more feasible when compared to a three-level factorial design. ${ }^{109}$

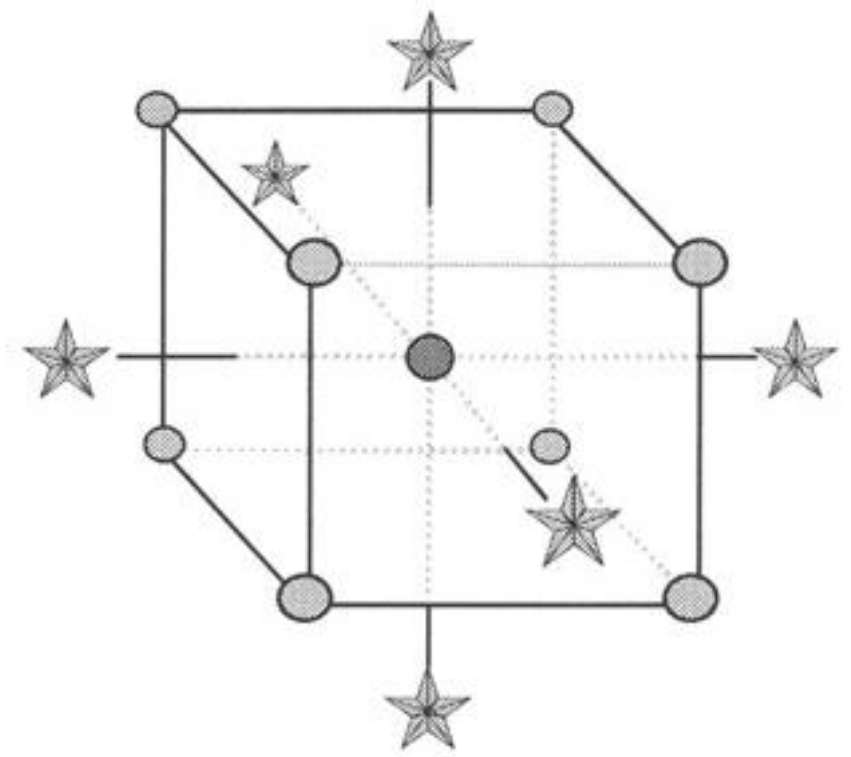

Figure 58: Depiction of a two-level CCD design space ${ }^{107}$ 
There are a number of different types of CCD, from the basic circumscribed design in which the star points lie outside the factorial box, to the inscribed design, which treats the factor settings as star points and so defines new test points within those limits. ${ }^{109}$ Finally, the face-centred CCD pulls the star points in so that they lie along the faces defined by the factorial ranges. ${ }^{107}$ For this study, the defined factor ranges represent the actual orientation limits attainable by the implant, and so the circumscribed CCD is not viable. Face-centred designs provide higher-quality predictions of surface curvature over the entire design space when compared to inscribed designs, and hence the face-centred CCD was used in this study.

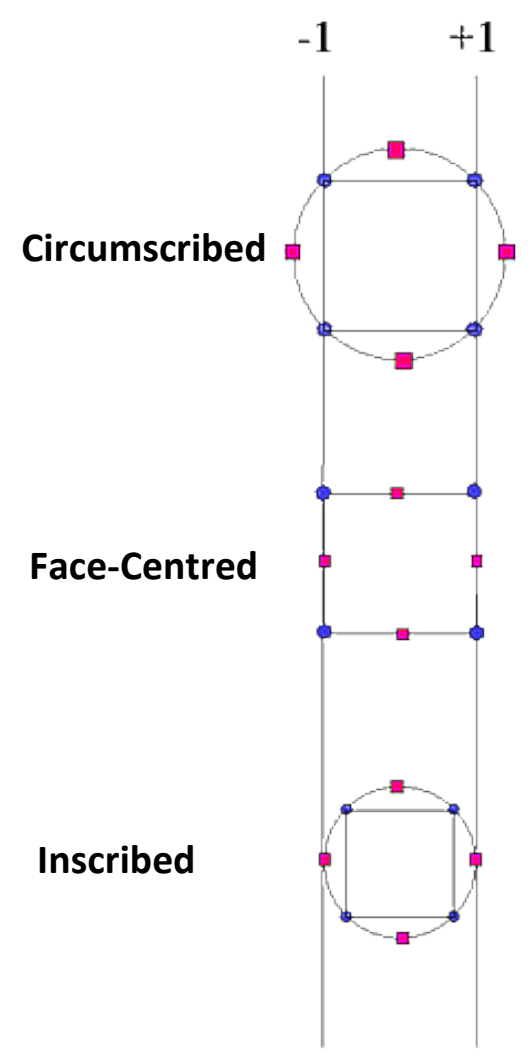

Figure 59: Comparison of the three types of CCD, indicating factorial ranges (blue) and star points (pink) ${ }^{109}$

\subsubsection{JMP for Design of Experiments}

The statistical software used for analysis in this study was JMP (SAS Institute Inc., Cary, NC, USA). This software allows the user to identify responses and factors, and then select the specific form of DOE desired. A list of the required experimental runs is then generated, with a 
corresponding input section for the predefined responses. The user can then run the model, which generates an array of analytic and diagnostic measures.

\subsubsection{JMP Design Parameters}

The first step in generating a CCD in JMP is to determine and input the factors to be analyzed. In order to account for variations in the units of measure, each of the predefined factor inputs used in this study was coded to a value of $+1,0$ or -1 (Table 7 ). They were furthermore given coded names for ease of analysis.

Table 7: CCD factor levels with corresponding coded names and values

\begin{tabular}{|clccc|}
\hline Coded Name & Factor & Low Level (-1) & Centre (0) & High Level (+1) \\
\hline A & Varus/Valgus $\left({ }^{\circ}\right)$ & -10 & 0 & 10 \\
\hline B & Anteversion $\left(^{\circ}\right)$ & 0 & 5 & 10 \\
\hline C & Posterior/Anterior $(\mathrm{mm})$ & -2 & 0 & 2 \\
\hline D & Inferior $(\mathrm{mm})$ & 0 & 2 & 4 \\
\hline
\end{tabular}

The responses chosen for use in this study were drawn from the regions identified in the Named Selections portion of Section 5.4.2.3. The exported values for the mean, standard deviation, and $90^{\text {th }}$ percentile stress of each of these regions were used in a number of combinations as response inputs, which have been described in detail in Section 5.5.3.2.

Typically, multiple centre points are used in CCD, as they give an indication of the between-run response variance of the system (pure error) and hence provide the DOF required for a lack of fit test. ${ }^{110}$ This test uses analysis of variance (ANOVA) to determine whether the proportion of error not explained by the model is significant. ${ }^{111}$ While this test is useful, there are numerous other means to assess the fit of the model to the data, including values for the correlation coefficient (R) and residual plots. For this study, a computer simulation was used to generate the responses of each run, and thus there was no variation between runs regardless of how many times the run was replicated. For that reason, an on face CCD with no replicates and 1 centre point was selected within the JMP DOE design box, giving rise to a design with 25 runs (Table 8). A prospective power analysis of this design $(\alpha=0.05)$ indicated that all main and two-factor interaction effects had strong predictive capabilities (power $>0.9$ ). 
Table 8: All of the runs specified by JMP given the chosen inputs, with a pattern indicating the composition of each run: "+" for high setting, "." for low setting, "A" high axial, "a" low axial, "0" for centre point ${ }^{111}$

\begin{tabular}{|c|c|c|c|c|c|}
\hline Run & Pattern & A & B & C & D \\
\hline 1 & ---- & -1 & -1 & -1 & -1 \\
\hline 2 & ---+ & -1 & -1 & -1 & 1 \\
\hline 3 & --+- & -1 & -1 & 1 & -1 \\
\hline 4 & --++ & -1 & -1 & 1 & 1 \\
\hline 5 & $\mathrm{a} 000$ & -1 & 0 & 0 & 0 \\
\hline 6 & -+-- & -1 & 1 & -1 & -1 \\
\hline 7 & -+-+ & -1 & 1 & -1 & 1 \\
\hline 8 & -++- & -1 & 1 & 1 & -1 \\
\hline 9 & -+++ & -1 & 1 & 1 & 1 \\
\hline 10 & Oa00 & 0 & -1 & 0 & 0 \\
\hline 11 & $00 \mathrm{aO}$ & 0 & 0 & -1 & 0 \\
\hline 12 & 000a & 0 & 0 & 0 & -1 \\
\hline 13 & 0000 & 0 & 0 & 0 & 0 \\
\hline 14 & $000 A$ & 0 & 0 & 0 & 1 \\
\hline 15 & OOAO & 0 & 0 & 1 & 0 \\
\hline 16 & OAOO & 0 & 1 & 0 & 0 \\
\hline 17 & +--- & 1 & -1 & -1 & -1 \\
\hline 18 & +--+ & 1 & -1 & -1 & 1 \\
\hline 19 & +-+- & 1 & -1 & 1 & -1 \\
\hline 20 & +-++ & 1 & -1 & 1 & 1 \\
\hline 21 & A000 & 1 & 0 & 0 & 0 \\
\hline 22 & ++-- & 1 & 1 & -1 & -1 \\
\hline 23 & ++-+ & 1 & 1 & -1 & 1 \\
\hline 24 & +++- & 1 & 1 & 1 & -1 \\
\hline 25 & ++++ & 1 & 1 & 1 & 1 \\
\hline
\end{tabular}

Another measure that can be used to assess the strength of a design is the alias matrix. Aliasing of effects occurs when a fractional design is used, which is often desirable when fewer runs are required due to experimental restrictions. A factor can have a confounding relationship, or exhibit aliasing with another factor, when its effects are attributed to that factor. For example, if a design uses the same sequence of factor settings to determine both a main effect and an interaction effect, then these two effects are aliased. This can cause problems if one of those factors happens to be a significant effect, as it could falsely label the other factor as significant as well. ${ }^{108}$ JMP provides a prospective diagnostic report that allows the user to determine whether 
any aliasing is present in the given model. In this study, it was found that only the third-order terms were aliased, with second-order terms exhibiting only mild correlations (Figure 60). This degree of aliasing was considered acceptable, particularly considering the rarity of three-factor interactions. $^{108,111}$

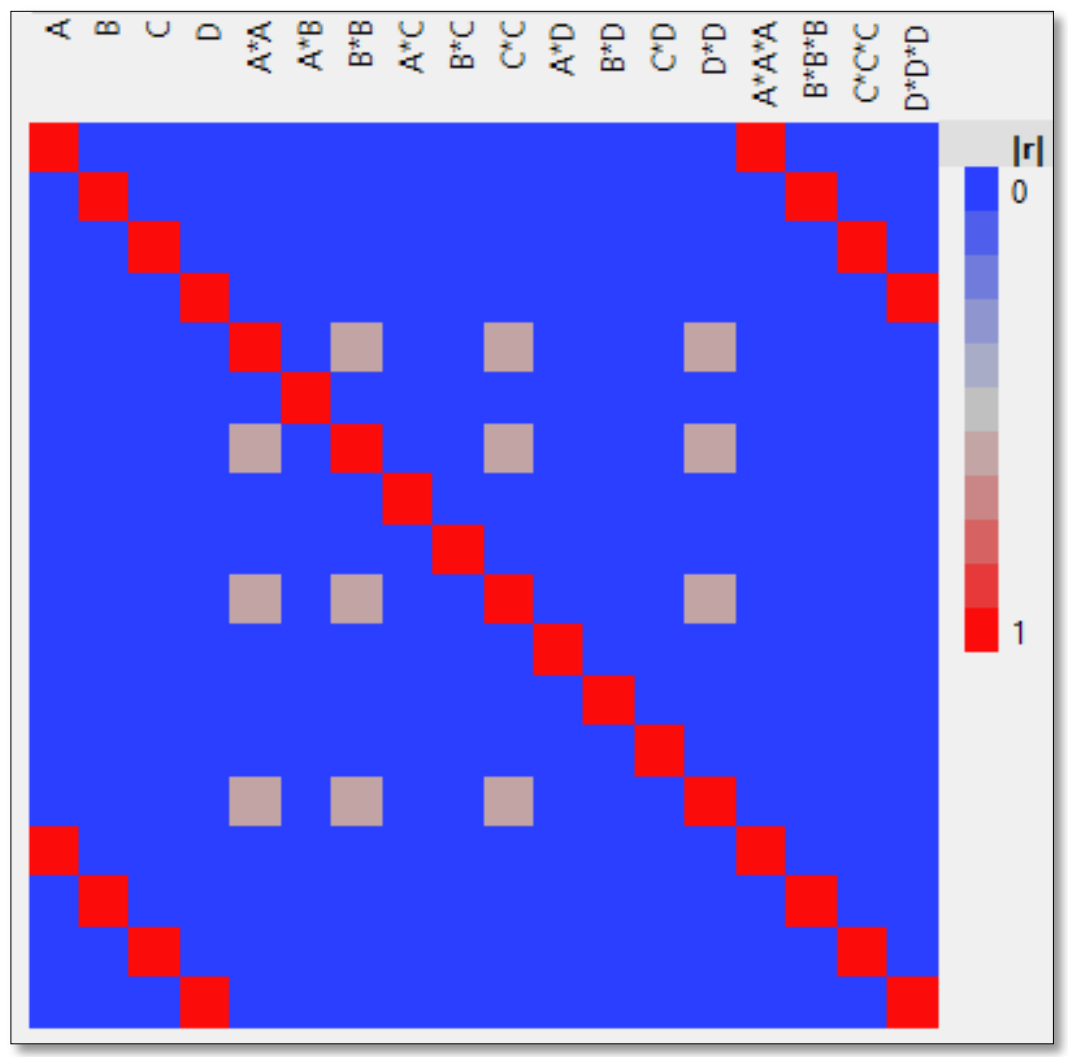

Figure 60: Colour map depicting the aliasing of effects, where a blue square indicates no correlation and a red square indicates complete aliasing

\subsubsection{Approaches to Response Selection}

The goal of the chosen outcome responses variables was to capture the variance in the stress distribution arising from each orientation. Three different approaches to estimate this response were therefore developed, with the objective of producing an optimality envelope (Table 9). In order to take into account the intact stress values and thereby ensure a comparative outcome model, there were two different methods used throughout each of these approaches. The first method (Method 1) involved comparing the raw stress values obtained from each region of interest to the corresponding intact stress value. In this case, the solution was optimized to match the intact value. The second method (Method 2) involved taking a percent of the intact value for 
each measure, which is a commonly used criterion when assessing femur stress in relation to a base value (Equation 3). ${ }^{31,42}$ These measures were subsequently optimized to match 100, which equated to the intact femur value. Each method was input into a separate DOE to allow for independent evaluation.

\section{Equation 3: Calculation of the percent of the intact femur, which was applied for each response measure in the second method}

$$
\% \text { of Intact }=\left(\frac{\text { Response Stress Measure }}{\text { Intact Value }}\right) * 100
$$

\section{Approach 1 - Stress Measures for Proximal Region Only}

The first approach assessed only the Proximal Region, as defined previously. The mean of the stress values within this region was determined to provide an idea of the central tendency of the stress level in this area. The standard deviation of these stresses was also calculated to give a sense of the spread of the data. Finally, the $90^{\text {th }}$ percentile stress value was found in order to evaluate the general range of the data. This was used in place of a maximum stress value, which was considered too unstable and subject to local modelling effects to be an apt measure. Each of these measures was optimized through DOE using both Method 1 (raw value) and Method 2 (percent of intact).

\section{Approach 2 - Mean Stress Values for All Regions}

The second approach utilized the other three regions of interest in addition to the Proximal Region to evaluate stress distribution within the proximal femur. In this method, the mean stress values developed in each of the Proximal, Inferior Neck, Superior Neck and Lateral Regions were input as response variables. It was theorized that the average stress value would give a good indication of the overall response in each region, including any exceptionally high or low values. Again, both input methods were used, assessing the optimal orientation generated by both the raw stress values and the percent of intact femur values.

\section{Approach 3 - $90^{\text {th }}$ Percentile Stress Values for All Regions}

The final approach utilized the $90^{\text {th }}$ percentile stress values for each of the defined regions of interest. The principle behind this approach was to assess the behaviour of $90 \%$ of the stress in a given region, without any directional bias that might be caused by the presence of local 
maximums. For this approach, the $90^{\text {th }}$ percentile was calculated for the Proximal, Inferior Neck, Superior Neck and Lateral Regions, and input as both a raw value and a percent of the intact femur value.

Table 9: Overview of each response selection approach and the method used to generate the employed input value

\begin{tabular}{|c|c|c|c|c|}
\hline Approach & Included Regions & Stress Measure & \multicolumn{2}{|c|}{ Input Value } \\
\hline \multirow{3}{*}{1} & \multirow{3}{*}{ Proximal } & Mean & \multirow{3}{*}{$\begin{array}{c}\text { Method } 1 \\
\text { (Raw Values) }\end{array}$} & \multirow{3}{*}{$\begin{array}{c}\text { Method } 2 \\
\text { (Percent of } \\
\text { Intact) }\end{array}$} \\
\hline & & Standard Deviation & & \\
\hline & & 90th Percentile & & \\
\hline \multirow{4}{*}{2} & Proximal & Mean & \multirow{4}{*}{$\begin{array}{c}\text { Method } 1 \\
\text { (Raw Values) }\end{array}$} & \multirow{4}{*}{$\begin{array}{c}\text { Method } 2 \\
\text { (Percent of } \\
\text { Intact) }\end{array}$} \\
\hline & Inferior & Mean & & \\
\hline & Superior & Mean & & \\
\hline & Lateral & Mean & & \\
\hline \multirow{4}{*}{3} & Proximal & 90th Percentile & \multirow{4}{*}{$\begin{array}{c}\text { Method } 1 \\
\text { (Raw Values) }\end{array}$} & \multirow{4}{*}{$\begin{array}{c}\text { Method } 2 \\
\text { (Percent of } \\
\text { Intact) }\end{array}$} \\
\hline & Inferior & 90th Percentile & & \\
\hline & Superior & 90th Percentile & & \\
\hline & Lateral & 90th Percentile & & \\
\hline
\end{tabular}

\subsubsection{Model Significance and Selection}

JMP offers a number of methods with which to analyze the fit of a given outcome model, and assess the significant coefficients and factor interactions. For this study, it was determined that the initial fit of the model would be established through evaluation of the model $\mathrm{R}$ value and root-mean-square error (RMSE) values, in conjunction with its whole model ANOVA generated $p$-value. ${ }^{110}$ A model that fits the data well would have an R close to 1, low RMSE values and a $p<0.05$. The plot of residuals versus predicted values was also generated to confirm the random distribution of the data.

Individual terms were then assessed through evaluation of the significance of their discrete ANOVA generated $p$-values. Factors that had a $p$-value greater than 0.05 were considered insignificant to the response model equation and so were removed from the model. However, insignificant terms were left in the full model if they were found to be present in a higher order significant term (main effects were never removed), if it was determined that the reduced model had an inferior fit (governed by its $\mathrm{R}$ and RMSE values), or if the reduced model had a detrimental effect on the residual distribution. ${ }^{110,112}$ 


\subsubsection{Optimal Orientation}

Determination of the optimal set of parameters was completed for each input method within each response approach, yielding a total of 6 optimized outcomes. Evaluation of the individual contribution of each factor to its respective outcome response, and the interaction between factors, was accomplished through two main JMP diagnostic tools: parameter estimates and the desirability tool.

\subsubsection{Factor Coefficients}

Through a standard least squares fit and partial ANOVA $F$-tests on the model terms, it was possible to determine parameter coefficient estimates with corresponding $95 \%$ confidence interval (CI) and standard error (SE) terms. Due to the previous coding of factors, these coefficients could be directly compared with one another, enabling assessment of relative factor contributions. A higher negative or positive coefficient coupled with a low $p$-value indicated a greater impact on the response model, while a coefficient with a high $p$-value, regardless of size, would have a lesser effect. ${ }^{110}$ The CI allowed for determination of the potential range of the factor contribution.

\subsubsection{Desirability Tool}

The desirability tool offered within the prediction profiler was used to optimize the response surface for all of the included response inputs simultaneously. This was accomplished through setting a desirability scale, which ranged from the least desirable $(\mathrm{d}=0)$ to the most desirable $(d=1)$ outcome. ${ }^{108}$ Depending on the response input method, the most desirable outcome was set to be either the specific intact femur value or 100, and the highest and lowest response input values were given a desirability of 0 . The desirability curve between these ranges was quadratic, with the peak equating to the optimal response, and each response input was given equal importance in the optimization calculations to ensure the results were not inadvertently biased. Desirability was then maximized using an internal iterative approach, and the optimal setting for each factor was produced, with the resultant response and its 95\% CI indicated (Figure 61). 


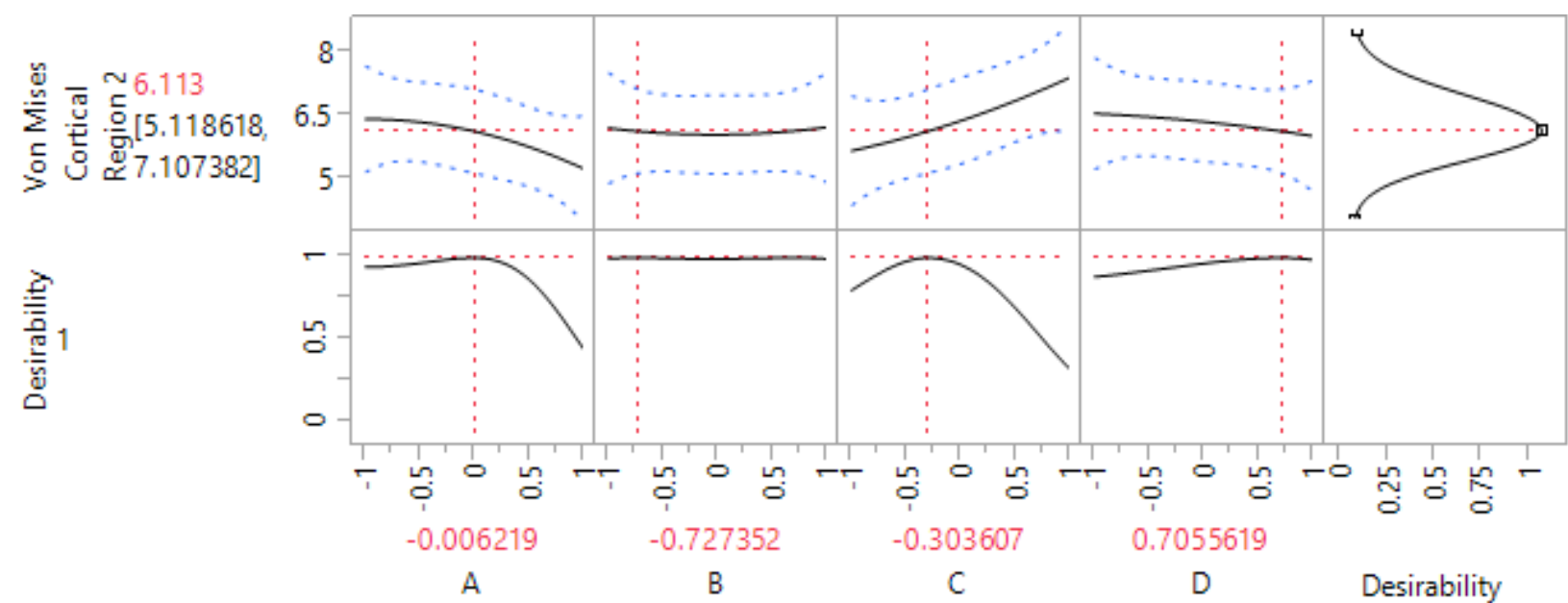

Figure 61: Screenshot of the desirability tool, where the lower graphs indicate the desirability response curve for each factor and the corresponding degree of desirability on the $y$-axis for each of the given factor settings, indicated by the numbers in red

\subsubsection{Definition of the Optimal Orientation}

As defined previously, an optimal orientation was generated from each approach and input method. A separate DOE was furthermore completed for each gait cycle phase, giving rise to 18 total optimized outcomes. A weighted average was used to determine the contribution of each optimal factor setting to the overall optimal orientation for each gait cycle phase. A weight was assigned to each factor depending upon the quantitative rise of its respective desirability response curve, in addition to the qualitative shape of its curve. Given that all desirability settings fell between 0 to 1 , this ensured uniformity of comparison between the varying approaches and methods. Furthermore, the shape was taken into account by multiplying the desirability weighting by 1 for a parabolic response, and by 1.5 for a linear response curve. For example, a linear desirability curve with a measured rise of 0.3 would be given a weight of 0.45 . A global optimal orientation was determined by averaging the weighted mean of each factor across all three gait cycle phases. The full weighting results for each gait cycle phase have been included in Appendix B.

A range of optimal settings for each factor was also acquired for each gait cycle phase, and used to generate optimal factor windows, within which biomechanical effects remained relatively stable. The optimal weighted average factor values for each gait cycle phase and the global optimal orientation were loaded at $3000 \mathrm{~N}$, representing 4 times body weight (BW) for a $75 \mathrm{~kg}$ 
person, which falls within the clinical-level range of $3-5$ times BW for hip joint contact forces during walking and jogging. ${ }^{83,113}$ The maximum stress developed under clinical-level loads was then evaluated to determine the potential for fracture at each optimal orientation.

\subsubsection{DOE Validation}

The success of the DOE was validated by testing the overall optimal orientation found for each gait cycle phase, and comparing the outcome stress responses to the design space corners. Each optimal orientation was therefore input into an FE model and loaded under $1000 \mathrm{~N}$, and the stresses developed in each Region were exported and analyzed. The generated response outcomes were then compared to the responses of each of the 25 runs in the respective gait cycle phase to ensure the optimality of the solution.

\subsubsection{Global Optimal Orientation Verification}

Once individual optimal orientations had been defined and validated against their respective DOE, the validity of the global optimal orientation had to be determined. This was accomplished through entering the global optimal orientation into an FE model and loading it under each of the neutral, extension, and flexion loading conditions. Resultant stress values in each Region of Interest were then compared to those values developed in the optimal orientation model for each respective gait cycle phase using the absolute value of the percentage difference (Equation 4).

Equation 4: Calculation of percentage difference (PD) using the intact (I) value and the equivalent implanted value $(X)$.

$$
P D=\left(\frac{I-X}{\frac{(I+X)}{2}}\right) * 100
$$




\section{CHAPTER 6}

Results

\subsection{Verification of Implant Orientation}

Prior to mechanical testing, all of the implanted femurs were plain film radiographed in an A/P and lateral view for verification of their correct implant location. In the A/P plane, it was determined that all of the stems were implanted to within $\pm 1^{\circ}$ of the planned CCD angle, with the exception of the varus orientation, which was measured to have a CCD angle of $115^{\circ}$ versus the planned $110^{\circ}$. This was therefore accounted for in the FE model, with experimental strains compared to a model with an implanted CCD angle of $115^{\circ}$. All of the lateral radiographs were assessed for stem version using the previously defined modified Resubal method, and were found to be within $\pm 2^{\circ}$ of their planned lateral angular position. Finally, all of the implanted femurs had $\mathrm{A} / \mathrm{P}$ and lateral positions within $\pm 1 \mathrm{~mm}$ of that planned.

\subsection{Validation of FE Model}

A linear regression analysis of the absolute value of the calculated experimental strains versus the absolute value of the FEM strains was completed in order to validate the model. Using JMP, a graph of experimental versus FEM strains was plotted, including strain data from all of the linear and rosette gauges, and the goodness of fit was assessed (Figure 62). Both the high correlation coefficient value of $\mathrm{R}=0.83$ and the small $p$-value of $p<0.0001$ indicated that there was a significant relationship between the measured and predicted values. ${ }^{83}, 114,115$ This was confirmed by assessing the plot of measured versus predicted residuals, which displayed a random distribution (Figure 63). It can therefore be concluded that the experimental and FEM strains displayed a linear relationship. 


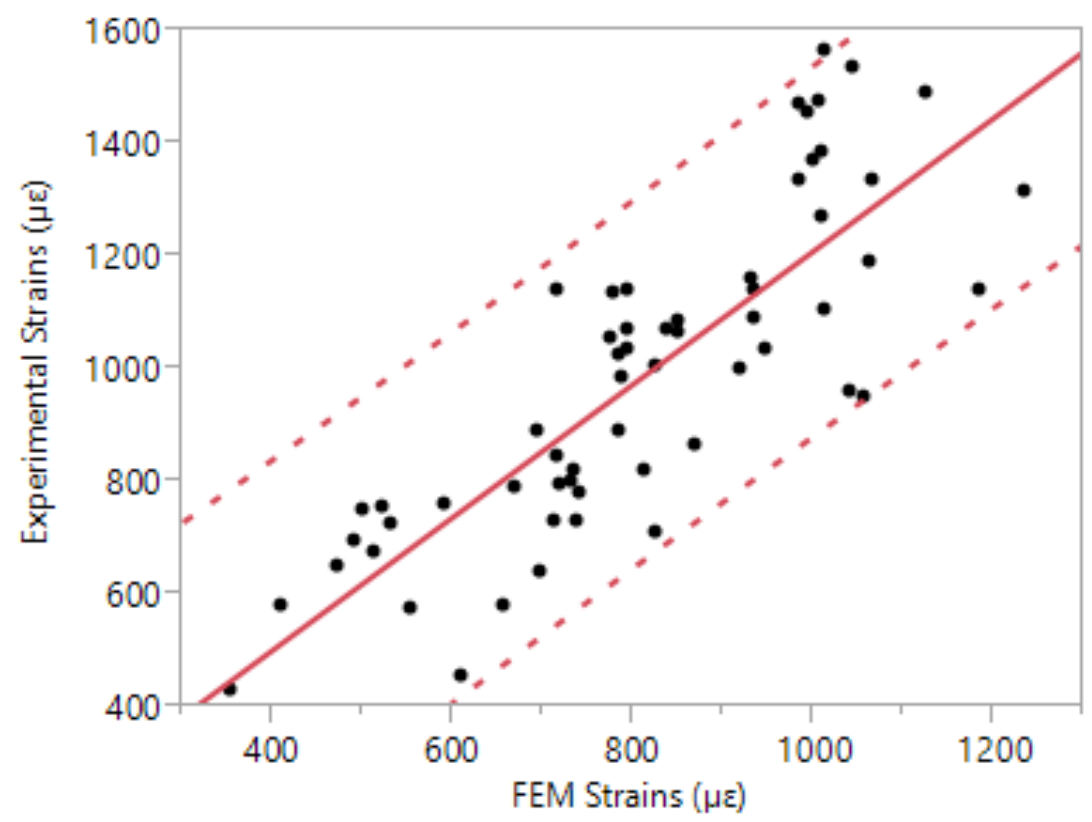

Figure 62: Correlation of all measured experimental and corresponding FEM strains including a line of best fit with $95 \%$ confidence limits. The equation of fit is $y=30.52+$ $1.18 x$, with a correlation coefficient of $R=0.83$, and $p<0.0001$

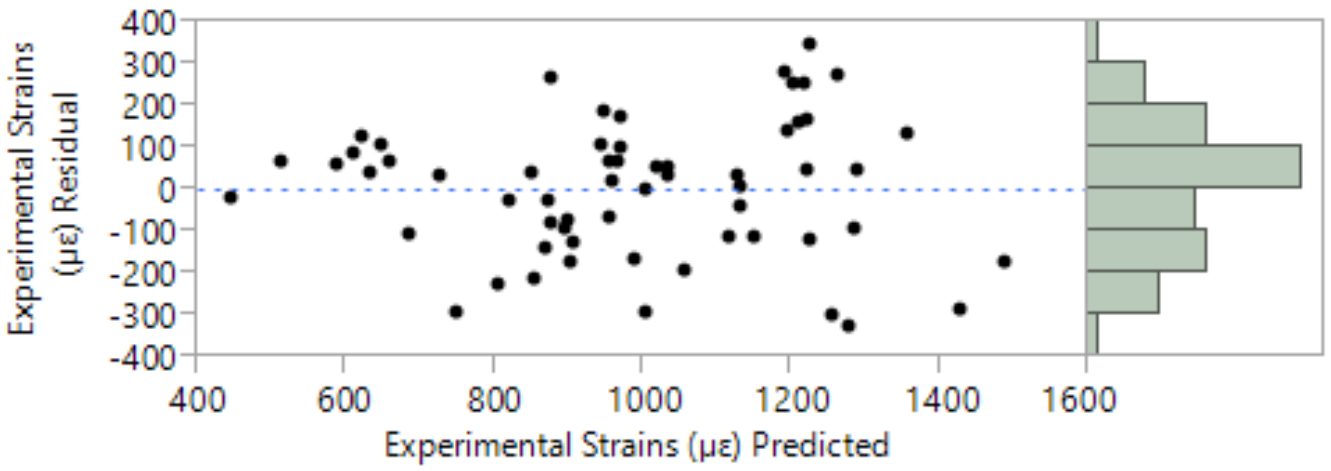

Figure 63: Residual plot of measured versus predicted strains

However, it should be noted that the slope of the regression line is equal to 1.18 instead of the desired 1, which suggests that the FE model is slightly stiffer than the experimental model, resulting in lower predicted strain values overall. This was confirmed by evaluating the stiffness resulting from the vertical deflection of the load application point in the FE model against the average of that produced experimentally for each implant orientation, where stiffness is calculated as the force $(\mathrm{N})$ over the displacement $(\mathrm{mm})$. A bar chart of the FEM and experimental stiffness values indicated that overall, experimental stiffness values equated to only about $50 \%$ of the FEM values (Figure 64). Nevertheless, these high FEM stiffness values are within the range of those reported in the literature for axially loaded synthetic femurs. ${ }^{83,116}$ 


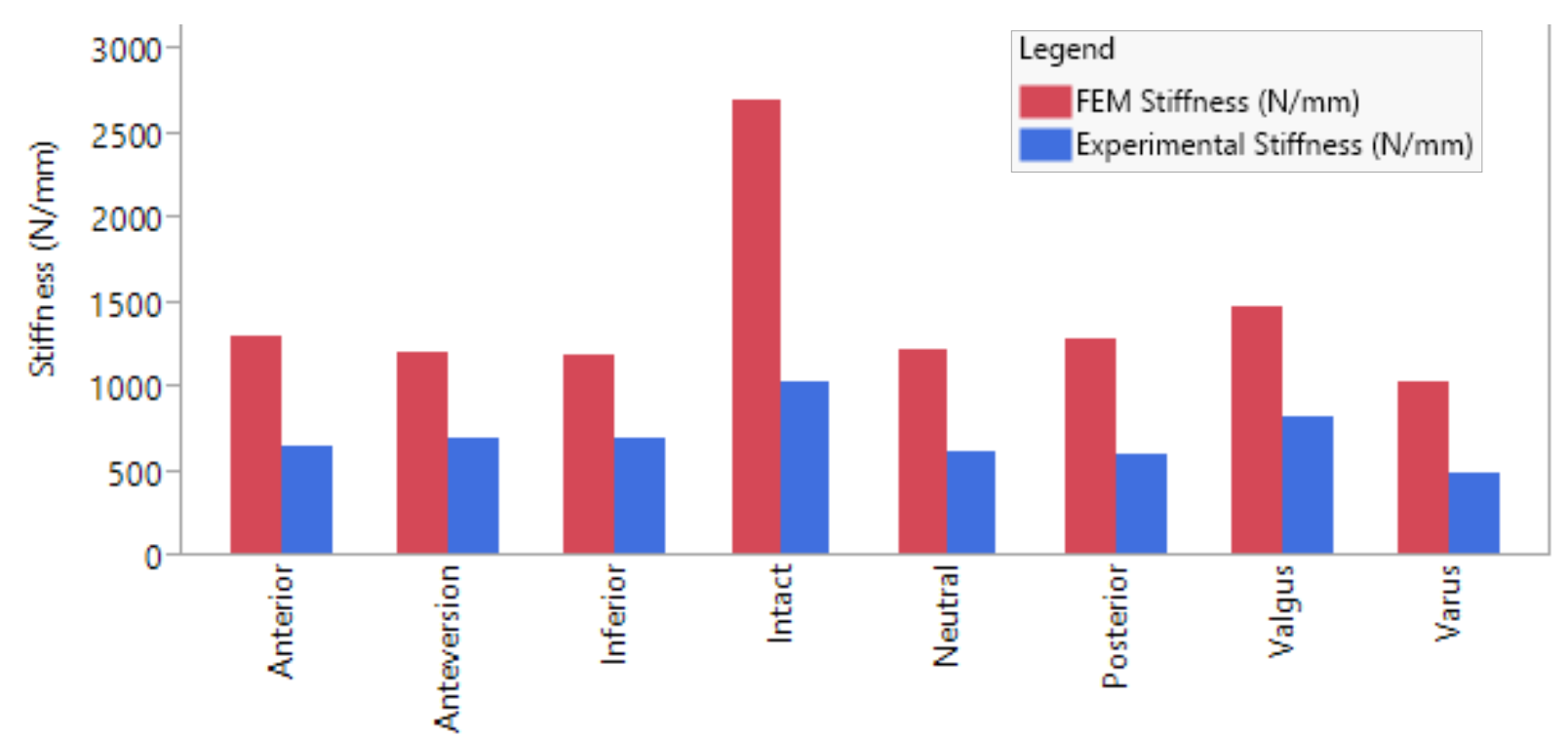

Figure 64: Bar chart of FEM and average experimental stiffness values for each implant orientation

\subsection{DOE Results}

For each stance condition (neutral, flexion, and extension), a list of the outcome responses for each input method has been provided, along with the terms included in the model and their coefficients and $p$-values. For a list of the response variable values input into each DOE, refer to Appendix A, and for full results of each approach see Appendices C, D, and E. It should be noted that throughout the results, each factor is referenced in its coded form, where A represents varus/valgus, B is anteversion, $\mathrm{C}$ denotes posterior/anterior, and D is inferior (Table 7).

\subsubsection{Neutral Phase}

\subsubsection{Approach 1 Method 1 Outcomes}

Responses corresponding to the mean stress, standard deviation, and $90^{\text {th }}$ percentile stress in the Proximal Region were analyzed initially. The intact stress values were always lower than the response values, so this model ultimately aimed to minimize the stress response. Through model evaluation it was determined that the model with insignificant terms removed yielded an improved overall significance, with no appreciable changes in the model fit. Furthermore, all of the residual plots were randomly distributed. The reduced model was therefore selected for analysis. 
The outcome of this model has been summarized, illustrating the 6 significant model terms: A, $\mathrm{B}, \mathrm{C}, \mathrm{D}, \mathrm{A} * \mathrm{~A}$ and $\mathrm{C}^{*} \mathrm{C}$ (Table 10). Factor B, or anteversion, was a significant factor in every outcome response, and was the most significant factor in two responses. In all instances, its positive coefficients indicate that as the degree of anteversion increased, the resultant stress values also increased, which is an unfavourable outcome. Factor A and its second order term were significant in two of the outcome responses. Taking into account that the varus/valgus factor spanned 0 (with negative varus and positive valgus), these coefficients therefore indicate that valgus reduced the stress response, while varus tended to increase the response. Similarly, the coefficients for factor $\mathrm{C}$ indicate that a negative posterior position tended to increase the stress response while it was decreased by the positive anterior position. Finally, factor D occurred in only one outcome response, and its positive coefficient indicates that increasing the inferior position caused a stress increase.

Table 10: Summary of the reduced Proximal Region stress model, sorted by significance, and indicating each response measure with its corresponding model terms, coefficients, and p-values. Significant terms have been marked with an asterisk (*) and bolded.

\begin{tabular}{|c|c|c|c|}
\hline $\begin{array}{l}\text { Outcome } \\
\text { Response }\end{array}$ & Term & Coded Coefficient & $p$-value \\
\hline \multirow{7}{*}{$\begin{array}{l}\text { Mean Stress } \\
\qquad(\mathrm{MPa})\end{array}$} & Overall Model & & $0.0014 *$ \\
\hline & B & 0.40 & $0.0033^{*}$ \\
\hline & $A * A$ & 0.77 & $0.0134 *$ \\
\hline & A & -0.31 & $0.016 *$ \\
\hline & $\mathrm{C}$ & -0.29 & $0.024 *$ \\
\hline & $C^{*} \mathrm{C}$ & -0.56 & 0.0626 \\
\hline & $\mathrm{D}$ & 0.23 & 0.0733 \\
\hline \multirow{7}{*}{$\begin{array}{l}\text { Stress Standard } \\
\text { Deviation (MPa) }\end{array}$} & Overall Model & & 0.0752 \\
\hline & B & 0.84 & $0.0203 *$ \\
\hline & C & -0.74 & $0.0379 *$ \\
\hline & $A^{*} A$ & 1.08 & 0.1846 \\
\hline & $C^{*} \mathrm{C}$ & -0.79 & 0.3274 \\
\hline & $\mathrm{D}$ & 0.26 & 0.4499 \\
\hline & A & -0.04 & 0.897 \\
\hline \multirow{7}{*}{$\begin{array}{l}\text { 90th Percentile } \\
\text { Stress (MPa) }\end{array}$} & Overall Model & & $<.0001 *$ \\
\hline & D & 1.26 & $<.0001^{*}$ \\
\hline & $\mathrm{C} * \mathrm{C}$ & 2.65 & $<.0001 *$ \\
\hline & A & -0.59 & $0.0047 *$ \\
\hline & C & 0.59 & $0.0047^{*}$ \\
\hline & B & 0.39 & $0.0471 *$ \\
\hline & $A^{*} A$ & -0.30 & 0.4893 \\
\hline
\end{tabular}


To confirm the results of the coefficient evaluation, the desirability tool was used to match the responses to the inputted intact femur values. The orientation yielding overall stress values most similar to the intact femur was found to occur at the following factor settings: $A=0.32, B=-1, C$ $=0.56, \mathrm{D}=-1$ (Figure 65). When converted from the coded values, this corresponds to an orientation of $3.15^{\circ}$ valgus, $0^{\circ}$ anteversion, $1.13 \mathrm{~mm}$ anterior, and $0 \mathrm{~mm}$ inferior. These results agree with the previous assessment of the effect of each factor on the stress response.

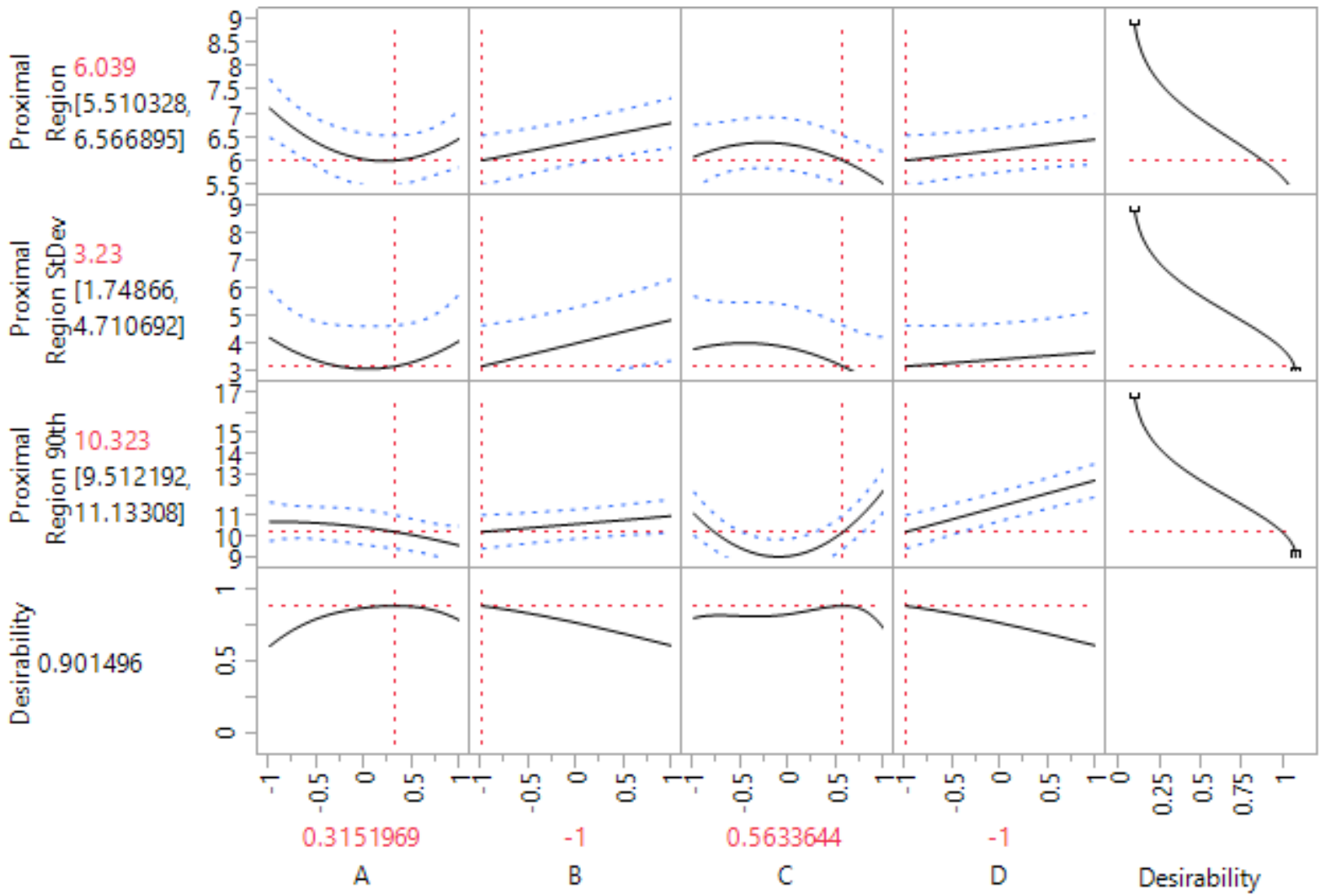

Figure 65: Output of the desirability tool for stress response, indicating ideal factor settings along the bottom row, with corresponding stress responses and CI along the left. Starting from the top row and going down, intact femur values for this optimization were 5.13MPa, 3MPa, and 9.19MPa

\subsubsection{Approach 1 Method 2 Outcomes}

A model utilizing the percent of the intact femur values as a response measure was also completed for the Proximal Region. This model aimed to match responses to a value of 100 , which would indicate the intact femur values. The reduced model was again selected for 
analysis, as removal of the insignificant terms was not found to significantly influence the fit of the model or its residual distribution.

The results of this model have been summarized (Table 11). There were 7 significant factor and factor interaction terms: $A, B, C, D, C * C, B * C$, and $C^{*} D$. The second order interaction of factor $\mathrm{C}$ was one of the most significant, and appeared in every outcome response. Since this term is a quadratic function with a high positive coefficient, it can therefore be concluded that any deviation into the positive or negative anterior or posterior orientations significantly increased the resulting percentage value, which indicates a higher stress level than the intact femur. Another term which appeared in every response model was factor D, or inferior. The positive coefficient in this case denotes an increasing percentage value with increasing inferior positioning. The significant $\mathrm{B}$ factor responded in a similar manner, with greater anteversion leading to a higher percentage value. The negative coefficient for factor A implies that any (negative) varus positioning led to an increase in the percentage value, which is again an unfavourable response. Finally, there are two interaction terms, which suggest an interaction between the anterior/posterior and inferior positions, and between the anteversion and anterior/posterior orientations. For the $C^{*} \mathrm{D}$ interaction, the negative coefficient implies that a decrease in outcome percentage was achieved with either a posterior and slightly inferior position, or with an anterior and greater inferior position. Similarly for the B*C term, a decreased percentage value was only achieved at low levels of anteversion and an anterior position, or a posterior orientation and high anteversion levels. 
Table 11: Summary of the reduced Proximal Region \% of intact femur model, sorted by significance, and indicating each response measure with its corresponding model terms, coefficients, and $p$-values. Significant terms have been marked with an asterisk (*) and bolded.

\begin{tabular}{|c|c|c|c|}
\hline $\begin{array}{l}\text { Outcome } \\
\text { Response }\end{array}$ & Term & Coded Coefficient & $p$-value \\
\hline \multirow{9}{*}{ Mean Stress (\%) } & \multicolumn{2}{|l|}{ Overall Model } & $<.0001 *$ \\
\hline & $\mathrm{C}^{*} \mathrm{C}$ & 23.70 & $<.0001 *$ \\
\hline & $A$ & -6.98 & $0.0003^{*}$ \\
\hline & D & 6.79 & $0.0004 *$ \\
\hline & C*D & -4.39 & $0.0162 *$ \\
\hline & $\mathrm{B}^{*} \mathrm{C}$ & 4.30 & $0.0182 *$ \\
\hline & B & 3.51 & $0.0369 *$ \\
\hline & $\mathrm{B}^{*} \mathrm{~B}$ & -6.01 & 0.1191 \\
\hline & $\mathrm{C}$ & 2.27 & 0.1608 \\
\hline \multirow{9}{*}{$\begin{array}{c}\text { Stress Standard } \\
\text { Deviation (\%) }\end{array}$} & \multicolumn{2}{|l|}{ Overall Model } & $<.0001 *$ \\
\hline & D & 13.66 & $<.0001^{*}$ \\
\hline & $\mathrm{C}^{*} \mathrm{C}$ & 29.10 & $<.0001^{*}$ \\
\hline & A & -6.38 & $0.0027 *$ \\
\hline & C & 6.38 & $0.0027^{*}$ \\
\hline & B & 4.21 & $0.0326 *$ \\
\hline & $B * C$ & 3.71 & 0.0697 \\
\hline & $C * D$ & -2.49 & 0.2107 \\
\hline & $B * B$ & -3.80 & 0.3861 \\
\hline \multirow{9}{*}{$\begin{array}{l}\text { 90th Percentile } \\
\text { Stress (\%) }\end{array}$} & \multicolumn{2}{|l|}{ Overall Model } & $0.0002^{*}$ \\
\hline & $\mathrm{C}^{*} \mathrm{C}$ & 95.62 & $<.0001 *$ \\
\hline & $C * D$ & -21.86 & $0.0094^{*}$ \\
\hline & $B * C$ & 18.95 & $0.0212 *$ \\
\hline & D & 15.88 & $0.0372 *$ \\
\hline & $\mathrm{C}$ & 13.42 & 0.0728 \\
\hline & $\mathrm{B} * \mathrm{~B}$ & -19.02 & 0.2677 \\
\hline & B & 7.44 & 0.3027 \\
\hline & A & 1.76 & 0.8042 \\
\hline
\end{tabular}

These results were confirmed using the desirability plots, which produced an optimal orientation at $\mathrm{A}=1, \mathrm{~B}=-1, \mathrm{C}=0.39$, and $\mathrm{D}=-1$ (Figure 66). This corresponds to an orientation of $10^{\circ}$ valgus, $0^{\circ}$ anteversion, $0.78 \mathrm{~mm}$ anterior, and $0 \mathrm{~mm}$ inferior. 


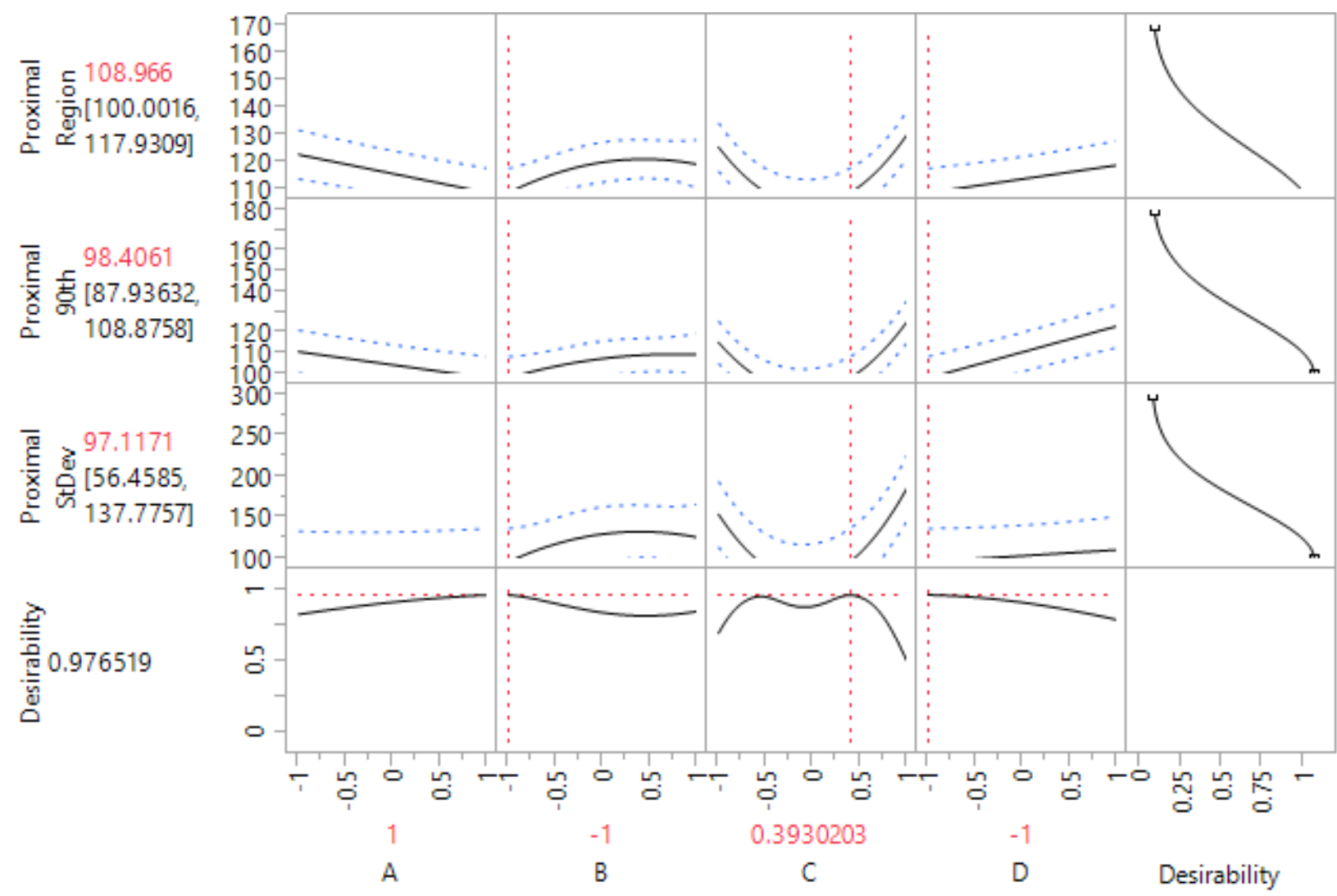

Figure 66: Output of the desirability tool for $\%$ of intact, indicating ideal factor settings along the bottom row, with corresponding stress responses and CI along the left. All intact femur values for this optimization corresponded to 100

\subsubsection{Approach 2 Method 1 Outcomes}

The second approach evaluated the stress distribution in the proximal femur through inclusion of the mean stress values for each defined Region. The goal of this model was to minimize the stress response (or match the intact femur value). The reduced model for this approach was selected even though some large decreases in the $\mathrm{R}$ values were noted, as it was determined that the statistical benefits provided by the increased DOF were more critical. There was furthermore no noticeable difference in the distribution of the residual plots.

There were only 4 significant terms resulting from the selected model: A, B, C, and B*C. Factor B was the most significant factor in two of the response models, with positive coefficients that suggest increasing anteversion gave rise to higher resultant stress values. Factor A was present in three response outcomes, with a negative coefficient indicating that a greater degree of varus caused an increased stress response. The lone significant $\mathrm{C}$ term denotes higher stress in the 
posterior position, while the interaction term $\mathrm{B}^{*} \mathrm{C}$ gives rise to a correlation in which stresses could only be minimized if the degree of anteversion increased when going from a posterior to anterior position.

Table 12: Summary of the reduced regional stress model, sorted by significance, and indicating each response measure with its corresponding model terms, coefficients, and $p$ values. Significant terms have been marked with an asterisk (*) and bolded.

\begin{tabular}{|c|c|c|c|}
\hline $\begin{array}{l}\text { Outcome } \\
\text { Response }\end{array}$ & Term & Coded Coefficient & $p$-value \\
\hline \multirow{7}{*}{$\begin{array}{l}\text { Proximal Region } \\
\text { Mean Stress } \\
\text { (MPa) }\end{array}$} & Overall Model & & $0.0066 *$ \\
\hline & B & 0.40 & $0.0066 *$ \\
\hline & A & -0.31 & $0.0269 *$ \\
\hline & C & -0.29 & $0.0384 *$ \\
\hline & $A^{*} A$ & 0.43 & 0.0958 \\
\hline & $\mathrm{D}$ & 0.23 & 0.1017 \\
\hline & $\mathrm{B}^{*} \mathrm{C}$ & -0.03 & 0.8367 \\
\hline \multirow{7}{*}{$\begin{array}{l}\text { Inferior Region } \\
\text { Mean Stress } \\
\text { (MPa) }\end{array}$} & Overall Model & & $0.2141 *$ \\
\hline & B & 1.25 & $0.0322 *$ \\
\hline & A & -0.88 & 0.1202 \\
\hline & $\mathrm{C}$ & -0.52 & 0.349 \\
\hline & $A^{*} A$ & 0.61 & 0.5578 \\
\hline & $B^{*} \mathrm{C}$ & -0.14 & 0.8032 \\
\hline & $D$ & -0.02 & 0.9673 \\
\hline \multirow{7}{*}{$\begin{array}{l}\text { Superior Region } \\
\text { Mean Stress } \\
\text { (MPa) }\end{array}$} & Overall Model & & $0.0201 *$ \\
\hline & $\mathrm{B} * \mathrm{C}$ & -0.53 & $0.011^{*}$ \\
\hline & A & -0.50 & $0.0111^{*}$ \\
\hline & $\mathrm{D}$ & -0.28 & 0.1242 \\
\hline & $\mathrm{C}$ & 0.22 & 0.2286 \\
\hline & $A^{*} A$ & -0.15 & 0.6663 \\
\hline & $\mathrm{B}$ & -0.02 & 0.8959 \\
\hline \multirow{7}{*}{$\begin{array}{l}\text { Lateral Region } \\
\text { Mean Stress } \\
(\mathrm{MPa})\end{array}$} & Overall Model & & $0.0458 *$ \\
\hline & A & -0.37 & $0.0018^{*}$ \\
\hline & $A^{*} A$ & 0.26 & 0.1901 \\
\hline & $\mathrm{B}$ & 0.09 & 0.405 \\
\hline & $\mathrm{C}$ & -0.05 & 0.6317 \\
\hline & $B * C$ & 0.04 & 0.7418 \\
\hline & $\mathrm{D}$ & 0.01 & 0.8915 \\
\hline
\end{tabular}

These correlation evaluations were confirmed in the optimal response parameters generated by the desirability tool (Figure 67). The optimal orientation was defined as factor settings of $\mathrm{A}=$ 
$0.81, \mathrm{~B}=-1, \mathrm{C}=0.16$, and $\mathrm{D}=-1$. This equates to an orientation of $8.05^{\circ}$ valgus, $0^{\circ}$ anteversion, $0.32 \mathrm{~mm}$ anterior and $0 \mathrm{~mm}$ inferior.

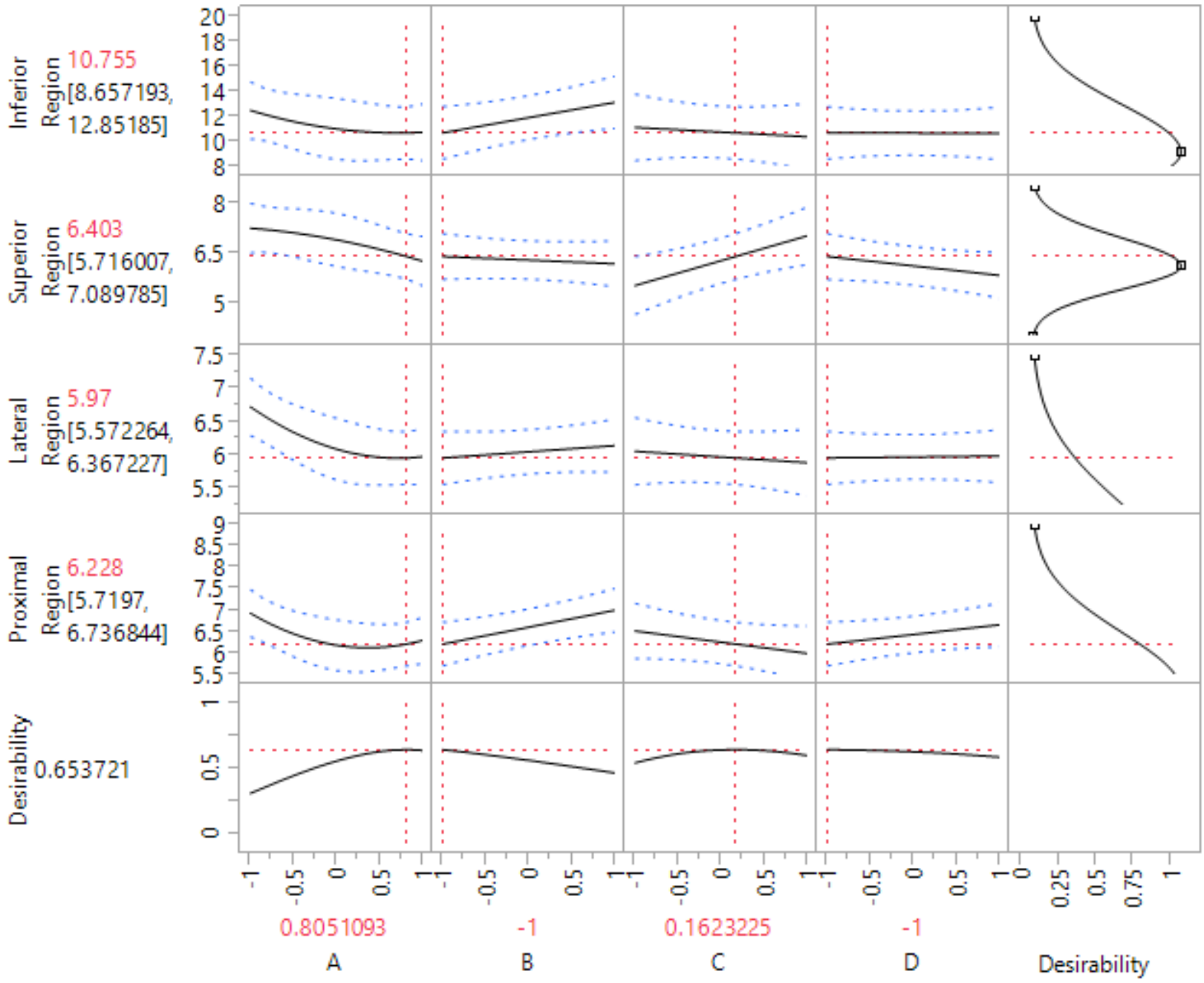

Figure 67: Output of the desirability tool for mean stress, indicating ideal factor settings along the bottom row, with corresponding stress responses and CI along the left. Starting from the top row and going down, the intact femur values for this optimization were 9.19MPa, 6.11 MPa, 4.06MPa and 5.13MPa

\subsubsection{Approach 2 Method 2 Outcomes}

When examining the effect of entering the percent of the intact values as a response outcome, it was determined that the reduced model had increased statistical power with no noticeable depreciation in the model fit or residual distribution. Hence insignificant terms were removed from the model. 
A summary of the model coefficients has been listed, with 8 terms standing out as significant: A, $\mathrm{B}, \mathrm{C}, \mathrm{D}, \mathrm{A}^{*} \mathrm{~B}, \mathrm{C}^{*} \mathrm{C}, \mathrm{C}^{*} \mathrm{D}$, and $\mathrm{B} * \mathrm{C}$ (Table 13$)$. The majority of these terms can be explained in a manner similar to that done previously, in which the varus orientation and increasing anteversion were found to yield higher percentage values, while any degree of anterior or posterior positioning significantly increased the percentage value. However, there were some conflicting terms with regard to the main factors $\mathrm{C}$ and $\mathrm{D}$, which have both positive and negative coefficients depending on the outcome response model. There was furthermore a new interaction term $\mathrm{A} * \mathrm{~B}$, which has a negative coefficient that indicates the percentage value decreased with increasing levels of valgus and anteversion.

These conflicting results were resolved through use of the desirability tool, which indicated the overall optimal orientation based on significance levels (Figure 68). The optimal factor settings were found to be $\mathrm{A}=1, \mathrm{~B}=-1, \mathrm{C}=-0.06$, and $\mathrm{D}=-0.94$. These correspond to an orientation with $10^{\circ}$ of valgus, $0^{\circ}$ of anteversion, $0.11 \mathrm{~mm}$ posterior, and $0.12 \mathrm{~mm}$ inferior. 
Table 13: Summary of the reduced regional \% of intact model, sorted by significance, and indicating each response measure with its corresponding model terms, coefficients, and $p$ values. Significant terms have been marked with an asterisk (*) and bolded.

\begin{tabular}{|c|c|c|c|}
\hline $\begin{array}{l}\text { Outcome } \\
\text { Response }\end{array}$ & Term & Coded Coefficient & $p$-value \\
\hline \multirow{9}{*}{$\begin{array}{l}\text { Proximal Region } \\
\text { Mean Stress (\%) }\end{array}$} & Overall Model & & $<.0001 *$ \\
\hline & $\mathrm{C}^{*} \mathrm{C}$ & 20.07 & $<.0001 *$ \\
\hline & $A$ & -6.98 & $0.0001 *$ \\
\hline & D & 6.79 & $0.0002 *$ \\
\hline & $C * D$ & -4.39 & $0.0086 *$ \\
\hline & $\mathrm{B} * \mathrm{C}$ & 4.30 & $0.0098 *$ \\
\hline & $A * B$ & -3.94 & $0.0163^{*}$ \\
\hline & B & 3.51 & $0.022 *$ \\
\hline & $\mathrm{C}$ & 2.27 & 0.1211 \\
\hline \multirow{9}{*}{$\begin{array}{l}\text { Inferior Region } \\
\text { Mean Stress (\%) }\end{array}$} & Overall Model & & $0.0049 *$ \\
\hline & $\mathrm{C}^{*} \mathrm{C}$ & 32.64 & $0.0009 *$ \\
\hline & $C * D$ & -13.23 & $0.0099 *$ \\
\hline & $B * C$ & 9.32 & 0.056 \\
\hline & $A$ & -8.16 & 0.0736 \\
\hline & $A * B$ & -5.93 & 0.2081 \\
\hline & $B$ & 4.73 & 0.2838 \\
\hline & $\mathrm{D}$ & 3.07 & 0.4823 \\
\hline & $\mathrm{C}$ & 0.04 & 0.9924 \\
\hline \multirow{9}{*}{$\begin{array}{l}\text { Superior Region } \\
\text { Mean Stress (\%) }\end{array}$} & Overall Model & & $<.0001 *$ \\
\hline & D & -10.84 & $<.0001 *$ \\
\hline & A & -8.81 & $<.0001 *$ \\
\hline & B & 5.77 & $0.0001 *$ \\
\hline & C & -4.92 & $0.0006 *$ \\
\hline & B*C & 3.54 & $0.0111 *$ \\
\hline & $C^{*} \mathrm{C}$ & -3.54 & 0.127 \\
\hline & $A * B$ & -1.24 & 0.3297 \\
\hline & $C * D$ & -1.07 & 0.3976 \\
\hline \multirow{9}{*}{$\begin{array}{l}\text { Lateral Region } \\
\text { Mean Stress (\%) }\end{array}$} & Overall Model & & $<.0001 *$ \\
\hline & A & -13.21 & $<.0001 *$ \\
\hline & C & 3.03 & $0.0002^{*}$ \\
\hline & D & 2.65 & $0.0008 *$ \\
\hline & B & 2.50 & 0.0013* \\
\hline & $C^{*} \mathrm{C}$ & -2.08 & 0.106 \\
\hline & $C * D$ & -0.56 & 0.4252 \\
\hline & $A * B$ & -0.51 & 0.4638 \\
\hline & $\mathrm{B}^{*} \mathrm{C}$ & -0.15 & 0.8312 \\
\hline
\end{tabular}




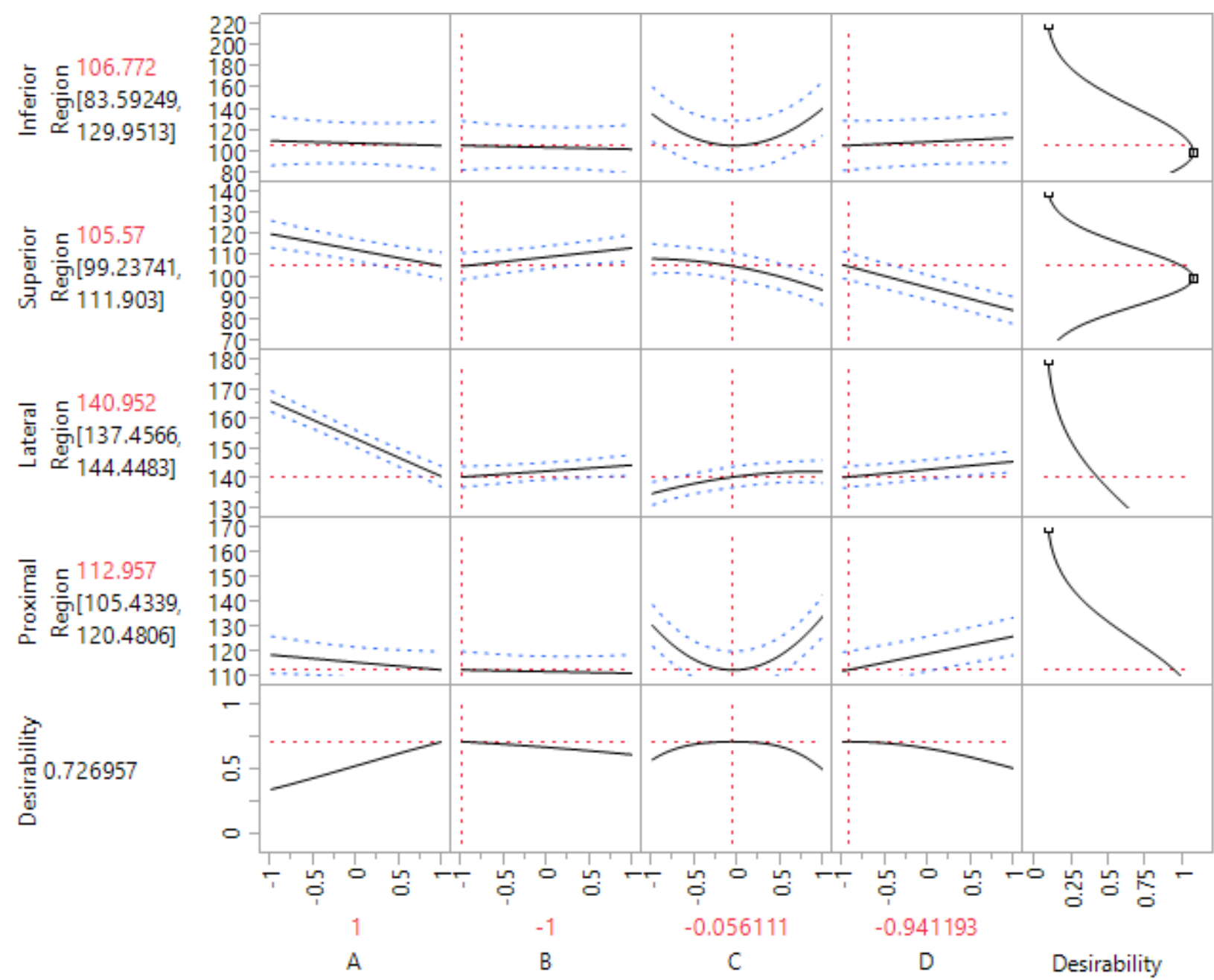

Figure 68: Output of the desirability tool for percent of intact stress, indicating ideal factor settings along the bottom row, with corresponding stress responses and $\mathrm{CI}$ along the left. All intact femur values for this optimization corresponded to 100

\subsubsection{Approach 3 Method 1 Outcomes}

The third approach involved looking at all of the regions of interest again; however, this time the $90^{\text {th }}$ percentile stress value was used as the response outcome. The reduced model was again selected for this approach, as there were no appreciable changes in the model fit or residuals, and the statistical power was seen to increase.

There were 8 significant main factors and factor interactions in the chosen model: A, B, C, D, $C^{*} \mathrm{C}, \mathrm{B} * \mathrm{C}, \mathrm{C}^{*} \mathrm{D}$, and $\mathrm{B}^{*} \mathrm{D}$ (Table 14). Most of the factors again followed similar trends in terms of their output responses, where greater varus and anteversion increased the stress response, 
while the anterior/posterior quadratic term produced unfavourable results in either direction. The inferior factor was also seen to have both positive and negative coefficients depending on the response region. However, there was a new significant two-factor interaction term $\mathrm{B} * \mathrm{D}$, for which the response stress could only be reduced if a higher inferior setting was subsequently combined with a decreased anteversion setting and vice versa.

This combination of coefficient requirements was optimized using the desirability tool, and the orientation yielding the greatest desirability was determined (Figure 69). The optimal factor settings were $\mathrm{A}=1, \mathrm{~B}=-1, \mathrm{C}=-0.21$, and $\mathrm{D}=-0.30$, which corresponds to an orientation of $10^{\circ}$ valgus, $0^{\circ}$ anteversion, $0.43 \mathrm{~mm}$ posterior, and $1.39 \mathrm{~mm}$ inferior. 
Table 14: Summary of the reduced regional $90^{\text {th }}$ percentile stress model, sorted by significance, and indicating each response measure with its corresponding model terms, coefficients, and $p$-values. Significant terms have been marked with an asterisk $\left(^{*}\right)$ and bolded.

\begin{tabular}{|c|c|c|c|}
\hline Outcome & Term & Coded Coefficient & $p$-value \\
\hline \multirow{9}{*}{$\begin{array}{c}\text { Proximal Region } \\
\text { 90th Percentile } \\
\text { Stress (MPa) }\end{array}$} & Overall Model & & $<.0001 *$ \\
\hline & $\mathrm{C} * \mathrm{C}$ & 2.46 & $<.0001 *$ \\
\hline & D & 1.26 & $<.0001^{*}$ \\
\hline & A & -0.59 & $0.003^{*}$ \\
\hline & c & 0.59 & $0.003^{*}$ \\
\hline & B & 0.39 & $0.0351 *$ \\
\hline & $B * C$ & 0.34 & 0.0738 \\
\hline & $C * D$ & -0.23 & 0.2176 \\
\hline & $B^{*} D$ & -0.09 & 0.6136 \\
\hline \multirow{9}{*}{$\begin{array}{c}\text { Inferior Region } \\
\text { 90th Percentile } \\
\text { Stress (MPa) }\end{array}$} & Overall Model & & $0.0007 *$ \\
\hline & $C * C$ & 11.77 & $<.0001^{*}$ \\
\hline & C*D & -3.93 & $0.0056 *$ \\
\hline & $B * C$ & 3.88 & $0.0061 *$ \\
\hline & C & 1.72 & 0.1568 \\
\hline & $B$ & 1.36 & 0.2574 \\
\hline & $\mathrm{D}$ & 0.76 & 0.5217 \\
\hline & $B^{*} D$ & -0.38 & 0.7632 \\
\hline & A & -0.31 & 0.7914 \\
\hline \multirow{9}{*}{$\begin{array}{c}\text { Superior Region } \\
\text { 90th Percentile } \\
\text { Stress (MPa) }\end{array}$} & Overall Model & & $<.0001 *$ \\
\hline & D & -1.15 & $<.0001^{*}$ \\
\hline & A & -0.81 & $<.0001 *$ \\
\hline & B & 0.48 & $0.0024^{*}$ \\
\hline & C & -0.41 & $0.0067^{*}$ \\
\hline & $B * C$ & 0.38 & $0.0158^{*}$ \\
\hline & $C^{*} \mathrm{C}$ & -0.31 & 0.2285 \\
\hline & $C * D$ & -0.11 & 0.4525 \\
\hline & $B * D$ & 0.09 & 0.5298 \\
\hline \multirow{9}{*}{$\begin{array}{l}\text { Lateral Region } \\
\text { 90th Percentile } \\
\text { Stress (MPa) }\end{array}$} & Overall Model & & $<.0001 *$ \\
\hline & A & -0.70 & $<.0001^{*}$ \\
\hline & D & 0.13 & $0.0013^{*}$ \\
\hline & B & 0.09 & $0.0177^{*}$ \\
\hline & $C * D$ & -0.08 & $0.0366 *$ \\
\hline & B*D & 0.08 & $0.0481 *$ \\
\hline & $C^{*} \mathrm{C}$ & -0.12 & 0.0773 \\
\hline & C & 0.06 & 0.1138 \\
\hline & $\mathrm{B}^{*} \mathrm{C}$ & -0.03 & 0.4294 \\
\hline
\end{tabular}




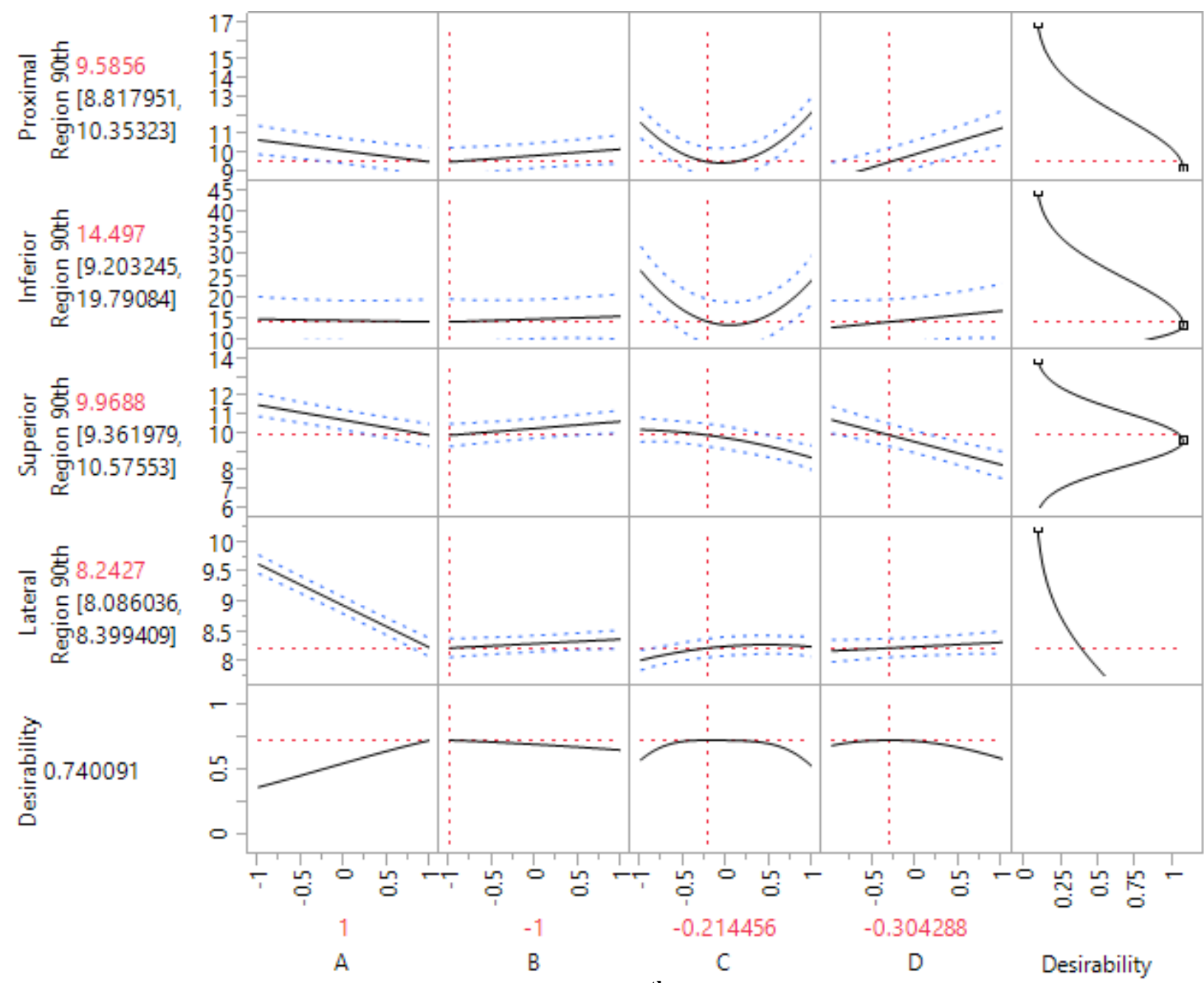

Figure 69: Output of the desirability tool for $90^{\text {th }}$ percentile stress, indicating ideal factor settings along the bottom row, with corresponding stress responses and CI along the left. Starting from the top row and going down, the intact femur values for this optimization were 9.19MPa, 13.61 MPa, 9.66MPa and 5.88MPa

\subsubsection{Approach 3 Method 2 Outcomes}

The final approach involved examining the $90^{\text {th }}$ percentile values as a percent of the intact femur. The full and reduced models were each assessed, and it was determined that insignificant variables would be removed, as there was little depreciation in the model fit or in the dispersion of residuals.

There were 7 significant terms in the analyzed model, which included factors: $A, B, C, D, C * C$, $\mathrm{B} * \mathrm{C}$, and $\mathrm{C} * \mathrm{D}$ (Table 15 ). Aside from a notably large and significant positive coefficient for the second order $\mathrm{C}$ term, there were no new factor effects noted in this model. In general, factor $\mathrm{A}$ 
had negative coefficients corresponding to an increasing percentage value with the varus angle, factor B had positive coefficients equating to larger percentages with increased anteversion, and factor D had both positive and negative significant coefficients.

Table 15: Summary of the reduced regional $90^{\text {th }}$ percentile $\%$ of intact stress model, sorted by significance, and indicating each response measure with its corresponding model terms, coefficients, and $p$-values. Significant terms have been marked with an asterisk (*) and bolded.

\begin{tabular}{|c|c|c|c|}
\hline $\begin{array}{l}\text { Outcome } \\
\text { Response }\end{array}$ & Term & Coded Coefficient & $p$-value \\
\hline \multirow{8}{*}{$\begin{array}{l}\text { Proximal Region } \\
\text { 90th Percentile } \\
\text { Stress (\%) }\end{array}$} & Overall Model & & $<.0001 *$ \\
\hline & $\mathrm{C}^{*} \mathrm{C}$ & 26.81 & $<.0001^{*}$ \\
\hline & D & 13.66 & $<.0001^{*}$ \\
\hline & A & -6.38 & $0.0024 *$ \\
\hline & $\mathrm{C}$ & 6.38 & $0.0024 *$ \\
\hline & B & 4.21 & $0.0309 *$ \\
\hline & $\mathrm{B}^{*} \mathrm{C}$ & 3.71 & 0.0671 \\
\hline & $C * D$ & -2.49 & 0.207 \\
\hline \multirow{8}{*}{$\begin{array}{l}\text { Inferior Region } \\
\text { 90th Percentile } \\
\text { Stress (\%) }\end{array}$} & Overall Model & & $0.0002 *$ \\
\hline & $\mathrm{C}^{*} \mathrm{C}$ & 86.45 & $<.0001 *$ \\
\hline & $C * D$ & -28.87 & $0.0044 *$ \\
\hline & $\mathrm{B} * \mathrm{C}$ & 28.50 & $0.0048 *$ \\
\hline & $\mathrm{C}$ & 12.65 & 0.1452 \\
\hline & $B$ & 10.00 & 0.2439 \\
\hline & $\mathrm{D}$ & 5.58 & 0.5099 \\
\hline & $\mathrm{A}$ & -2.29 & 0.7856 \\
\hline \multirow{8}{*}{$\begin{array}{l}\text { Superior Region } \\
\text { 90th Percentile } \\
\text { Stress (\%) }\end{array}$} & Overall Model & & $<.0001^{*}$ \\
\hline & D & -11.92 & $<.0001 *$ \\
\hline & A & -8.38 & $<.0001 *$ \\
\hline & B & 4.96 & $0.0019^{*}$ \\
\hline & C & -4.28 & $0.0057^{*}$ \\
\hline & $\mathrm{B} * \mathrm{C}$ & 3.93 & $0.0138 *$ \\
\hline & $\mathrm{C} * \mathrm{C}$ & -3.25 & 0.2194 \\
\hline & $C * D$ & -1.12 & 0.4437 \\
\hline \multirow{8}{*}{$\begin{array}{l}\text { Lateral Region } \\
\text { 90th Percentile } \\
\text { Stress (\%) }\end{array}$} & Overall Model & & $<.0001 *$ \\
\hline & A & -11.95 & $<.0001^{*}$ \\
\hline & D & 2.26 & $0.0026^{*}$ \\
\hline & B & 1.54 & $0.0278 *$ \\
\hline & $C * D$ & -1.41 & 0.0536 \\
\hline & $\mathrm{C} * \mathrm{C}$ & -2.08 & 0.1046 \\
\hline & $\mathrm{C}$ & 0.98 & 0.1463 \\
\hline & $B * C$ & -0.50 & 0.471 \\
\hline
\end{tabular}


The desirability tool output generally supported the discussed factor effects, recommending factor settings of $\mathrm{A}=1, \mathrm{~B}=-1, \mathrm{C}=-0.16$ and $\mathrm{D}=-0.38$ (Figure 70). This resulted in an optimal orientation at $10^{\circ}$ valgus, $0^{\circ}$ anteversion, $0.31 \mathrm{~mm}$ posterior, and $1.23 \mathrm{~mm}$ inferior.

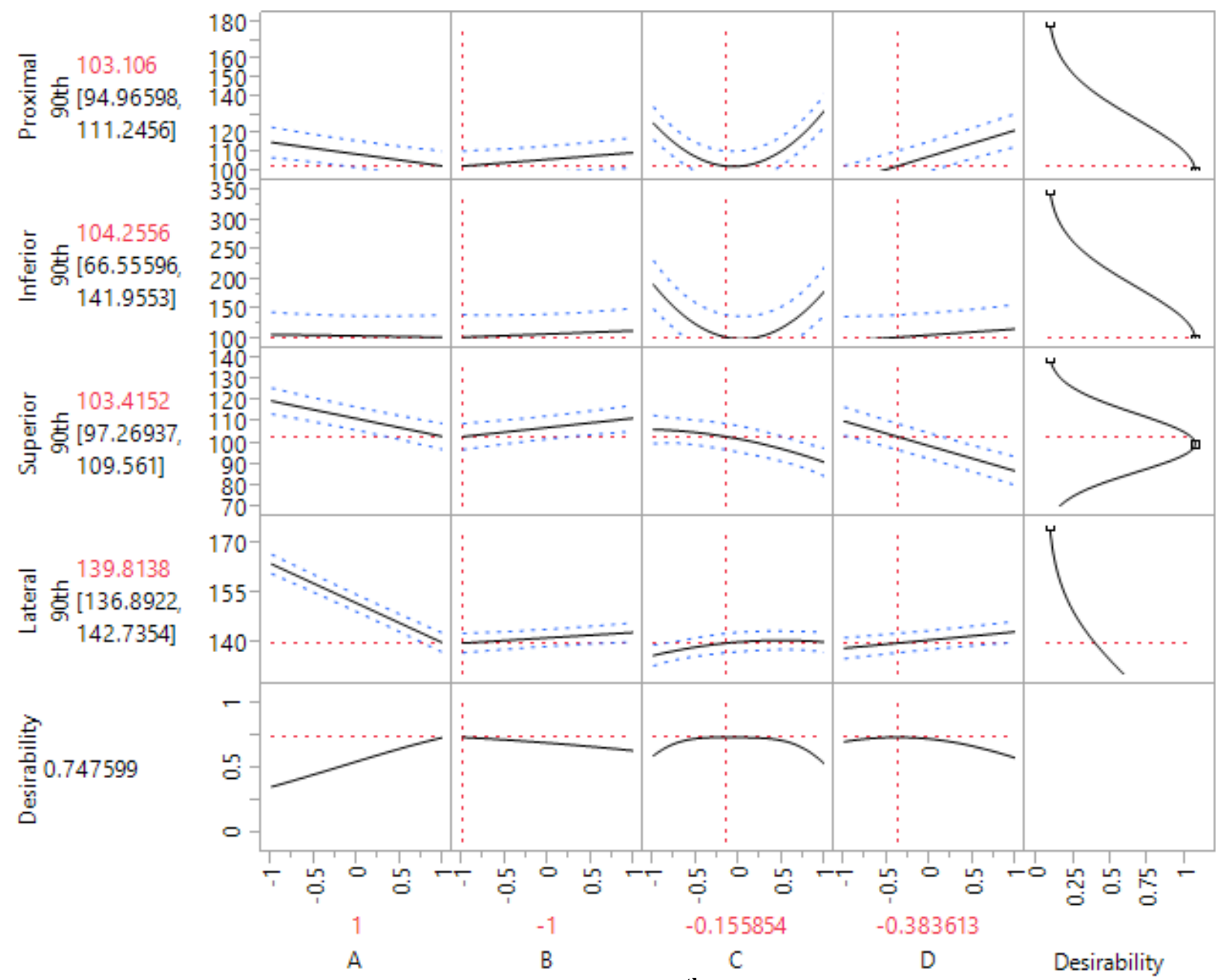

Figure 70: Output of the desirability tool for $90^{\text {th }}$ percentile $\%$ of intact femur stress, indicating ideal factor settings along the bottom row, with corresponding stress responses and $\mathrm{CI}$ along the left. All intact femur values for this optimization corresponded to 100

\subsubsection{Optimal Window}

In general, there were a number of factors that were seen to be consistently detrimental to either the absolute stress value or the percent of intact femur value. These crucial terms have been summarized and their implications on the outcome response described (Table 16). 
Table 16: Summary of common significant neutral terms and their corresponding effects, in addition to the region which was most significantly affected by the term

\begin{tabular}{|c|c|c|c|c|c|c|}
\hline $\begin{array}{l}\text { Significant } \\
\text { Term }\end{array}$ & $\begin{array}{l}\text { Coefficient } \\
\text { Sign }\end{array}$ & Implication & Proximal & Inferior & Superior & Lateral \\
\hline A & Negative & $\begin{array}{l}\text { Increasing varus produced higher } \\
\text { stresses }\end{array}$ & & & & $x$ \\
\hline B & Positive & $\begin{array}{l}\text { Increasing anteversion produced } \\
\text { higher stresses }\end{array}$ & $x$ & & $x$ & \\
\hline $\mathrm{B}^{*} \mathrm{C}$ & Positive & $\begin{array}{l}\text { If any anteversion present, an } \\
\text { anterior position produced higher } \\
\text { stresses }\end{array}$ & & $x$ & & \\
\hline$C * C$ & Positive & $\begin{array}{l}\text { Any deviations from the neutral } \\
\text { lateral position produced higher } \\
\text { stresses }\end{array}$ & $x$ & $x$ & & \\
\hline$C * D$ & Negative & $\begin{array}{l}\text { Any combination of anterior with } \\
\text { minimal inferior or posterior with } \\
\text { greater inferior produced higher } \\
\text { stresses }\end{array}$ & & $x$ & & \\
\hline D & $\begin{array}{l}\text { Positive } \\
\text { and } \\
\text { Negative }\end{array}$ & $\begin{array}{l}\text { Increasing inferior produced higher } \\
\text { stresses in all Regions except for } \\
\text { the Superior Region }\end{array}$ & $x$ & $x$ & $x$ & $x$ \\
\hline
\end{tabular}

In addition to the tabulated critical factors, all of the optimality settings have been compiled and the weighted means and setting ranges determined, yielding an optimality envelope (Table 17).

Table 17: Compilation of all optimal orientations generated by each neutral phase response model, specifying the weighted average values for each factor and the factor range

Response Selection Varus/Valgus $\left({ }^{\circ}\right) \quad$ Anteversion $\left({ }^{\circ}\right)$ Posterior/Anterior (mm) Inferior (mm)

\begin{tabular}{|lcccc|}
\hline Approach 1 Method 1 & 3.15 & 0.00 & 1.13 & 0.00 \\
\hline Approach 1 Method 2 & 10.00 & 0.00 & 0.78 & 0.00 \\
\hline Approach 2 Method 1 & 8.05 & 0.00 & 0.32 & 0.00 \\
\hline Approach 2 Method 2 & 10.00 & 0.00 & -0.11 & 0.12 \\
\hline Approach 3 Method 1 & 10.00 & 0.00 & -0.43 & 1.39 \\
\hline Approach 3 Method 2 & 10.00 & 0.00 & -0.31 & 1.23 \\
\hline Weighted Average & $\mathbf{8 . 9 0}$ & $\mathbf{0 . 0 0}$ & $\mathbf{0 . 1 1}$ & $\mathbf{0 . 4 0}$ \\
\hline Range & $\mathbf{3 . 1 5 - 1 0}$ & $\mathbf{0 . 0 0}$ & $\mathbf{- 0 . 4 3 - 1 . 1 3}$ & $\mathbf{0 - 1 . 3 9}$ \\
\hline
\end{tabular}

\subsubsection{FE Model of Neutral Optimal Orientation}

To allow for visual comparison between the specified neutral optimal orientation and the intact

FE model, the weighted average optimality settings for the neutral phase (Table 17) were entered into an FE model and loaded at a clinically realistic value of $3000 \mathrm{~N}$, then compared to the intact femur model loaded at 3000N (Figure 71). The maximum stress developed in the optimal 
orientation was evaluated in relation to the ultimate strength of the synthetic cortical bone in compression to ensure that there was no potential for failure.
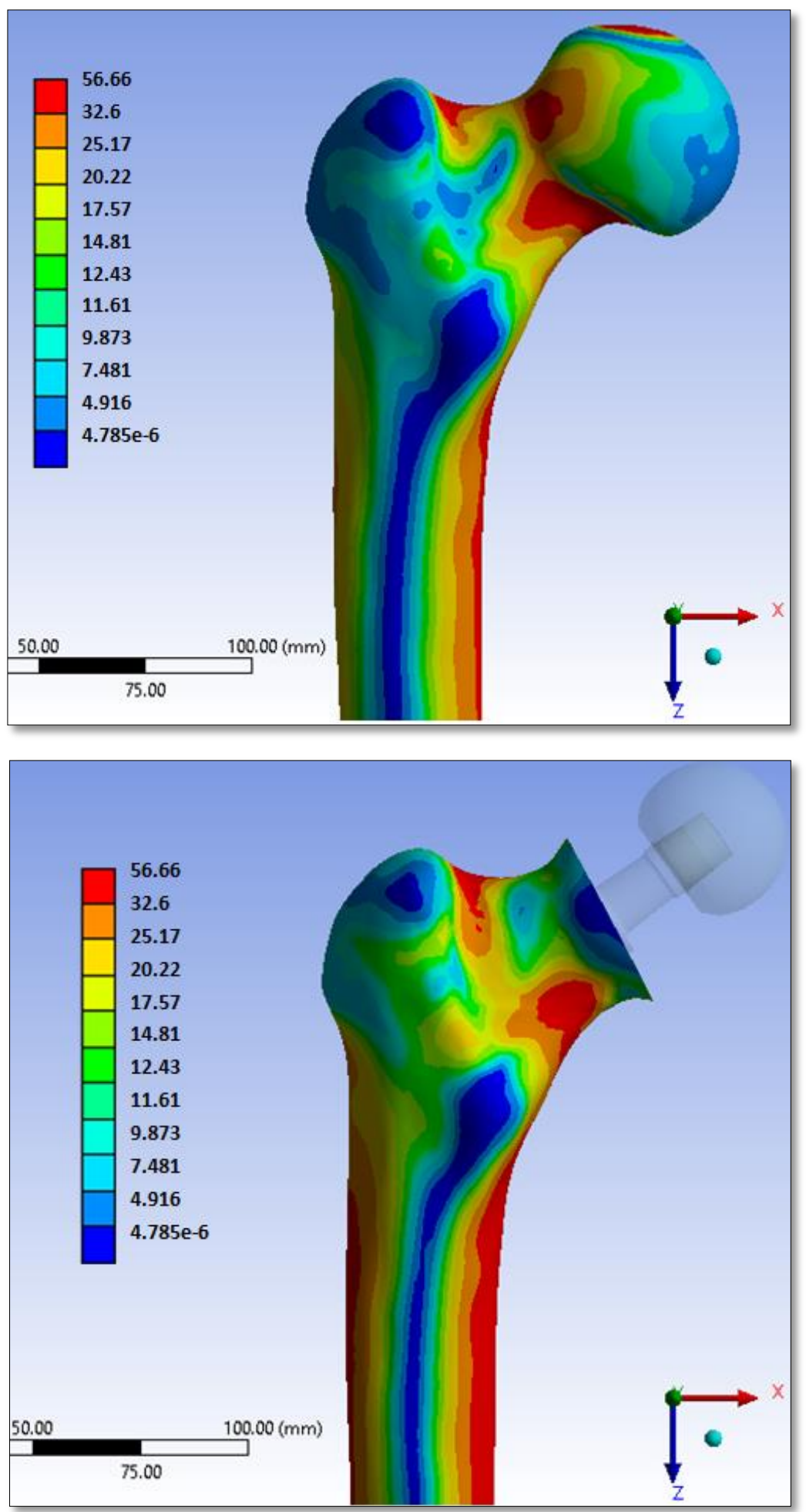

Figure 71: FE model of the intact femur loaded at 3000N (top), and the neutral phase optimally implanted femur loaded at $3000 \mathrm{~N}$ (bottom), equivalently scaled in von Mises stresses (MPa) for comparison purposes 
The maximum cortical bone stress in the optimally oriented implanted model was found to be $56.61 \mathrm{MPa}$, which is well below the manufacturer provided ultimate strength of synthetic cortical bone of $106 \mathrm{MPa}$, indicating no risk for fracture. ${ }^{63}$ Furthermore, the implanted femur exhibited a stress distribution similar to that of the intact femur, with comparable regions of maximum and minimum stress and a Proximal Region average stress of $115.7 \%$ of the intact femur value.

\subsubsection{Extension Phase}

In order to evaluate the behaviour of the implanted stem throughout the range of the gait cycle, the stress distribution at the toe-off phase, represented by $15^{\circ}$ of extension, was assessed. In a manner identical to the neutral stance, a DOE for each response selection approach and input method was completed, and the resulting factor coefficients evaluated (Table 18). Reduced models without insignificant terms were used for every response model except for Approach 2 Method 1, for which the removal of insignificant terms was found to have a drastic effect on the model fit. Using the match target function, all of the models were also optimized to match either the intact femur values or 100, depending on the method in use. The optimal orientation window was then determined (Table 19). For complete results see Appendix D.

Table 18: Summary of common significant extension terms and their corresponding effects, in addition to the region which was most significantly affected by the term

\begin{tabular}{|c|c|c|c|c|c|c|}
\hline $\begin{array}{l}\text { Significant } \\
\text { Term }\end{array}$ & $\begin{array}{l}\text { Coefficient } \\
\text { Sign }\end{array}$ & Implication & Proximal & Inferior & Superior & Lateral \\
\hline A & Negative & $\begin{array}{l}\text { Increasing varus orientation } \\
\text { produced higher stresses }\end{array}$ & & & $x$ & $x$ \\
\hline B & Positive & $\begin{array}{l}\text { Increasing anteversion produced } \\
\text { higher stresses }\end{array}$ & & & $x$ & $x$ \\
\hline$B^{*} C$ & Positive & $\begin{array}{l}\text { Increasing anteversion with } \\
\text { increasing anterior produced } \\
\text { higher stresses }\end{array}$ & & $x$ & & \\
\hline C & Positive & $\begin{array}{l}\text { Increasing anterior produced } \\
\text { higher stresses }\end{array}$ & $x$ & & & $x$ \\
\hline$C^{*} \mathrm{C}$ & Positive & $\begin{array}{l}\text { Any deviations from the neutral } \\
\text { lateral position produced higher } \\
\text { stresses }\end{array}$ & $x$ & $x$ & & \\
\hline D & $\begin{array}{c}\text { Positive and } \\
\text { Negative }\end{array}$ & $\begin{array}{l}\text { Increasing inferior produced } \\
\text { higher stresses in all Regions } \\
\text { except for the Superior Region }\end{array}$ & $x$ & $x$ & $x$ & $\mathrm{X}$ \\
\hline
\end{tabular}


Table 19: Compilation of all optimal orientations generated by each extension phase response model, specifying the weighted average values for each factor and the factor range Response Selection Varus/Valgus $\left(^{\circ}\right)$ Anteversion ( ${ }^{\circ}$ ) Posterior/Anterior (mm) Inferior (mm)

\begin{tabular}{|lcccc|}
\hline Approach 1 Method 1 & -0.98 & 0.00 & -0.17 & 0.00 \\
\hline Approach 1 Method 2 & -0.88 & 0.00 & -0.17 & 0.00 \\
\hline Approach 2 Method 1 & 10.00 & 0.00 & -0.82 & 0.00 \\
\hline Approach 2 Method 2 & 10.00 & 1.89 & -0.44 & 0.00 \\
\hline Approach 3 Method 1 & 10.00 & 0.00 & -0.93 & 0.00 \\
\hline Approach 3 Method 2 & 10.00 & 0.00 & -0.91 & 0.00 \\
\hline Weighted Average & $\mathbf{7 . 9 9}$ & $\mathbf{0 . 2 7}$ & $\mathbf{- 0 . 4 6}$ & $\mathbf{0 . 0 0}$ \\
\hline Range & $\mathbf{- 0 . 9 8 - 1 0}$ & $\mathbf{0 . 0 0 - 1 . 8 9}$ & $\mathbf{- 0 . 9 3 - - 0 . 1 7}$ & $\mathbf{0 . 0 0}$ \\
\hline
\end{tabular}

In order to evaluate the behaviour of the implant under clinical level loads in the extension condition, the optimal orientation was entered into an FE model and loaded at 3000N. The maximum stress developed was found to be $70.11 \mathrm{MPa}$, which is again well below the $106 \mathrm{MPa}$ ultimate strength value of synthetic cortical bone, as provided by the manufacturer. ${ }^{63}$ The implanted femur furthermore exhibited an average Proximal Region stress value of $112.4 \%$ of the intact femur, indicating good similarity to the intact condition.

\subsubsection{Flexion Phase}

The flexion phase represented the heel strike condition within the gait cycle, and corresponded to $15^{\circ}$ of flexion. Similar to the previous two phases, FEA was used to complete a DOE for the flexion condition and the resultant stress values were used as outcome responses in their raw form and as percent of the intact femur. All of the models used for this phase were reduced and so had their whole model insignificant variables removed. Resulting factor coefficients and terms were evaluated for their significance and implication in the overall stress reduction objective (Table 20). Using the desirability tool, an optimal orientation was determined for each model and a subsequent optimality range obtained (Table 21). For full results see Appendix E. 
Table 20: Summary of common significant flexion terms and their corresponding effects, in addition to the region which was most significantly affected by the term

\begin{tabular}{|c|c|c|c|c|c|c|}
\hline $\begin{array}{l}\text { Significant } \\
\text { Term }\end{array}$ & $\begin{array}{l}\text { Coefficient } \\
\text { Sign }\end{array}$ & Implication & Proximal & Inferior & Superior & Lateral \\
\hline$A$ & Negative & $\begin{array}{l}\text { Increasing varus orientation } \\
\text { produced higher stresses }\end{array}$ & $x$ & & $x$ & $x$ \\
\hline$A^{*} D$ & Negative & $\begin{array}{l}\text { Increasing valgus with lower } \\
\text { levels of inferior, or varus with } \\
\text { higher levels of inferior, } \\
\text { produced increased stresses }\end{array}$ & $x$ & & & $x$ \\
\hline$B^{*} \mathrm{C}$ & Positive & $\begin{array}{l}\text { Increasing anteversion with } \\
\text { increasing anterior produced } \\
\text { higher stresses }\end{array}$ & & $x$ & $x$ & \\
\hline C & Negative & $\begin{array}{l}\text { Increasing posterior produced } \\
\text { higher stresses }\end{array}$ & & & $x$ & \\
\hline $\mathrm{C}^{*} \mathrm{C}$ & Positive & $\begin{array}{l}\text { Any deviation from neutral } \\
\text { produced higher stress }\end{array}$ & $x$ & $x$ & & \\
\hline D & $\begin{array}{l}\text { Positive } \\
\text { and } \\
\text { Negative }\end{array}$ & $\begin{array}{l}\text { Increasing inferior produced } \\
\text { higher stresses in all Regions } \\
\text { except for the Superior Region }\end{array}$ & & & $x$ & \\
\hline
\end{tabular}

Table 21: Compilation of all optimal orientations generated by each flexion phase response model, specifying the weighted average values for each factor and the factor range

\begin{tabular}{|lcccc|}
\hline Response Selection & Varus/Valgus $^{\mathbf{(}^{\circ}}$ ) & Anteversion $\mathbf{(}^{\circ}$ ) & Posterior/Anterior (mm) & Inferior (mm) \\
\hline Approach 1 Method 1 & 10.00 & 10.00 & 0.00 & 1.88 \\
\hline Approach 1 Method 2 & 10.00 & 10.00 & 0.01 & 1.85 \\
\hline Approach 2 Method 1 & 10.00 & 10.00 & -1.55 & 0.00 \\
\hline Approach 2 Method 2 & 10.00 & 10.00 & -1.55 & 0.00 \\
\hline Approach 3 Method 1 & 10.00 & 10.00 & -1.38 & 0.90 \\
\hline Approach 3 Method 2 & 10.00 & 10.00 & -1.38 & 0.89 \\
\hline Weighted Average & $\mathbf{1 0 . 0 0}$ & $\mathbf{1 0 . 0 0}$ & $\mathbf{- 0 . 7 4}$ & $\mathbf{0 . 4 1}$ \\
\hline Range & $\mathbf{1 0 . 0 0}$ & $\mathbf{1 0 . 0 0}$ & $\mathbf{- 1 . 5 5 - 0 . 0 0}$ & $\mathbf{0 . 0 0 - 1 . 8 8}$ \\
\hline
\end{tabular}

The optimal orientation for the flexion condition was entered into an FE model and loaded under $3000 \mathrm{~N}$ to assess the stress resulting under clinical-level loads. The maximum stress was found to be 53.59MPa, which is below the manufacturer-stated ultimate strength of synthetic cortical bone of 106MPa, signifying no risk for fracture. ${ }^{63}$ The Proximal Region was also found to yield an average stress value of $103.3 \%$ of the intact femur, indicating good similarity to the intact condition.

\subsubsection{Global Optimal Orientation}

The global optimal orientation was calculated by taking an average of all of the output values for each factor setting, generating an orientation that features settings from the entire range of the 
gait cycle (Table 22). For comparison purposes, this global optimal orientation was entered into an FE model and loaded in single-leg stance at 3000N. The resultant stress distribution was again compared to the intact femur, while the maximum stress developed in the optimally implanted model was compared to the ultimate strength limits provided by the synthetic femur manufacturer (Figure 72).

Table 22: Global optimal orientation, including weighted average values for each factor and the total acceptable factor range

\begin{tabular}{|lcccc|}
\hline $\begin{array}{l}\text { Measure } \\
\text { Global Weighted } \\
\text { Average }\end{array}$ & Varus/Valgus $\left(^{\circ}\right)$ & Anteversion $\left({ }^{\circ}\right)$ & Posterior/Anterior (mm) & Inferior (mm) \\
\hline Range & 9.14 & 2.49 & -0.48 & 0.23 \\
\hline
\end{tabular}

The maximum cortical bone stress developed in the global optimally implanted femur was found to be $56.01 \mathrm{MPa}$, which is again well below the $106 \mathrm{MPa}$ ultimate strength limit for synthetic cortical bone, indicating no potential for failure. ${ }^{63}$ In addition to the neutral phase, the global optimal orientation was also entered into flexion and extension phase FE models and compared to corresponding intact femur models. It was found that the average stress value in the Proximal Region was $115.8 \%$ of intact under the neutral gait phase condition, $112.3 \%$ of intact under extension, and $108.1 \%$ of intact in the flexion condition, signifying a strong similarity to the intact femur throughout the range of the gait cycle. 

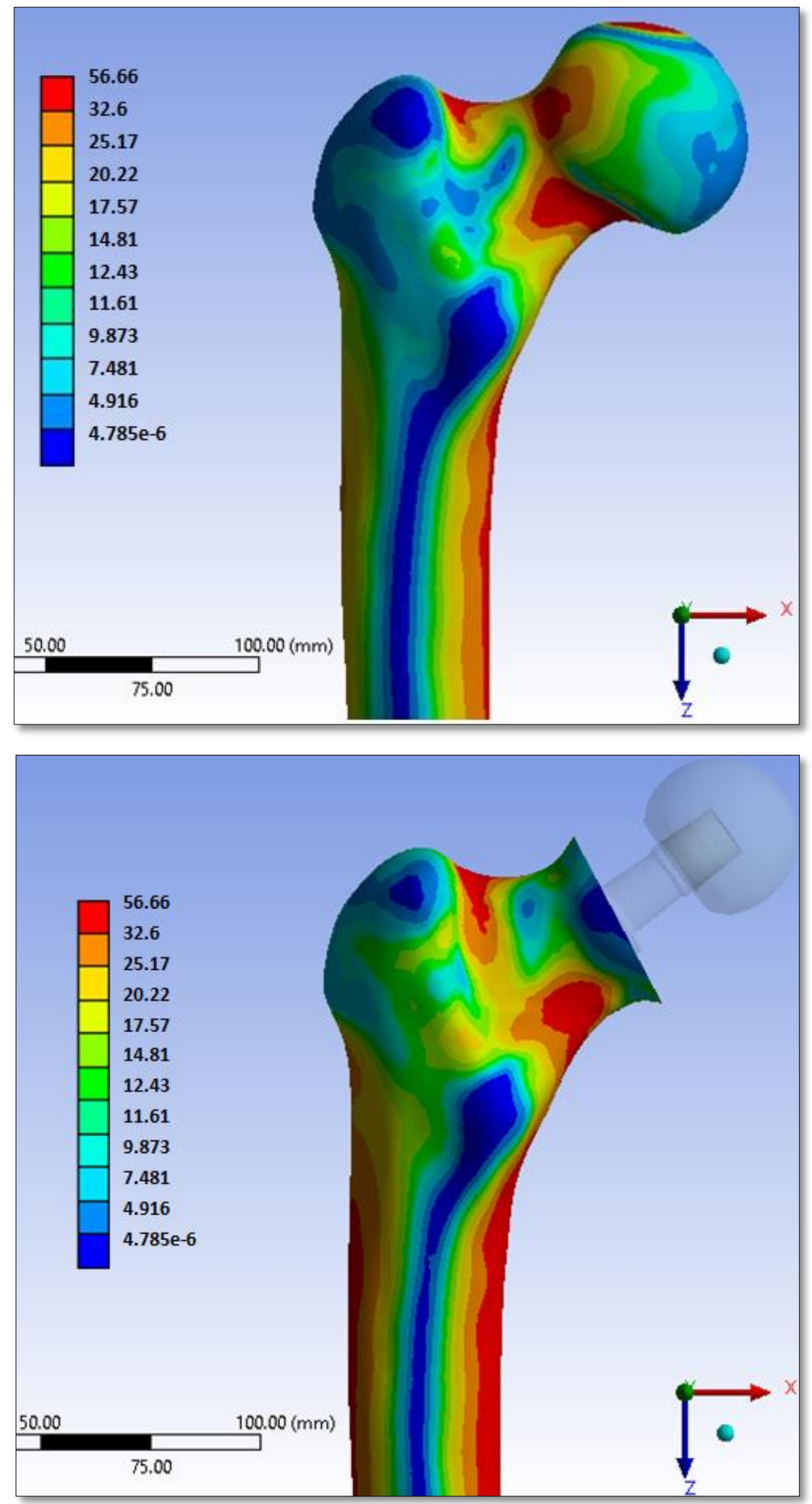

Figure 72: FE model of the intact femur loaded at $3000 \mathrm{~N}$ (top), and the global optimally implanted femur loaded at 3000N (bottom), equivalently scaled in von Mises stresses (MPa) for comparison purposes 


\subsection{Validation of DOE}

In order to evaluate the success of the DOE, the optimal orientation for each gait cycle phase was entered into an FE model and loaded under $1000 \mathrm{~N}$. The stress values produced in each Region of Interest were then retrieved and compared to those developed during each run of the respective gait phase DOE (Table 23).

Table 23: Response outcome measures for each Region of Interest across all gait phase optimal orientations, including the global optimal orientation, for loading under $1000 \mathrm{~N}$. Bolded and starred terms indicate responses that are more similar to the intact femur value than any other response outcome measure for that particular DOE

\begin{tabular}{|c|c|c|c|c|c|c|c|c|c|}
\hline $\begin{array}{c}\text { Gait } \\
\text { Phase }\end{array}$ & $\begin{array}{l}\text { Proximal } \\
\text { 90th }\end{array}$ & $\begin{array}{l}\text { Proximal } \\
\text { Average }\end{array}$ & $\begin{array}{l}\text { Proximal } \\
\text { Standard } \\
\text { Deviation }\end{array}$ & $\begin{array}{l}\text { Inferior } \\
\text { 90th }\end{array}$ & $\begin{array}{l}\text { Inferior } \\
\text { Average }\end{array}$ & $\begin{array}{l}\text { Superior } \\
\text { 90th }\end{array}$ & $\begin{array}{l}\text { Superior } \\
\text { Average }\end{array}$ & $\begin{array}{l}\text { Lateral } \\
\text { 90th }\end{array}$ & $\begin{array}{l}\text { Lateral } \\
\text { Average }\end{array}$ \\
\hline $\begin{array}{l}\text { Neutral } \\
\text { (MPa) }\end{array}$ & $9.85^{*}$ & $5.93 *$ & $2.78 *$ & $12.75^{*}$ & $8.77^{*}$ & 10.54 & 6.37 & 8.29 & 5.79 \\
\hline $\begin{array}{l}\text { Neutral } \\
(\%)\end{array}$ & $107.20 *$ & $115.72 *$ & 92.84 & 93.64 & 95.44 & 109.09 & 104.20 & 141.05 & 142.65 \\
\hline $\begin{array}{l}\text { Extension } \\
\text { (MPa) }\end{array}$ & $11.65^{*}$ & $6.85^{*}$ & $3.47^{*}$ & 14.84 & 10.08 & $11.55^{*}$ & $7.24 *$ & 8.81 & 6.02 \\
\hline $\begin{array}{l}\text { Extension } \\
\text { (\%) }\end{array}$ & $108.43^{*}$ & $112.35^{*}$ & $102.33^{*}$ & 90.08 & 95.40 & 101.62 & 101.00 & 131.40 & 129.70 \\
\hline $\begin{array}{l}\text { Flexion } \\
\text { (MPa) }\end{array}$ & $9.68 *$ & $5.63 *$ & 2.94 & 13.06 & 8.69 & 10.07 & 6.10 & 7.90 & 5.25 \\
\hline $\begin{array}{l}\text { Flexion } \\
\text { (\%) }\end{array}$ & 98.61* & $103.33 *$ & 87.92 & 90.76 & 86.97 & 99.03 & 96.07 & 127.22 & 130.30 \\
\hline $\begin{array}{l}\text { Global } \\
\text { Neutral } \\
(\mathrm{MPa})\end{array}$ & 9.87 & 5.94 & 2.78 & 12.69 & 8.75 & 10.93 & 6.64 & 8.25 & 5.71 \\
\hline $\begin{array}{l}\text { Global } \\
\text { Neutral } \\
(\%)\end{array}$ & 107.35 & 115.81 & 92.74 & 93.20 & 95.24 & 113.12 & 108.63 & 140.46 & 140.63 \\
\hline $\begin{array}{l}\text { Global } \\
\text { Extension } \\
\text { (MPa) }\end{array}$ & 11.70 & 6.84 & 3.50 & 14.94 & 10.14 & 11.18 & 7.07 & 8.76 & 6.00 \\
\hline $\begin{array}{l}\text { Global } \\
\text { Extension } \\
\text { (\%) }\end{array}$ & 108.92 & 112.29 & 103.32 & 90.70 & 95.95 & 98.39 & 98.59 & 130.69 & 129.23 \\
\hline $\begin{array}{l}\text { Global } \\
\text { Flexion } \\
\text { (MPa) }\end{array}$ & 10.09 & 5.89 & 3.11 & 13.49 & 9.16 & 10.58 & 6.35 & 8.24 & 5.32 \\
\hline $\begin{array}{l}\text { Global } \\
\text { Flexion } \\
\text { (\%) }\end{array}$ & 102.80 & 108.12 & 92.90 & 93.79 & 91.62 & 104.08 & 100.13 & 132.58 & 131.93 \\
\hline
\end{tabular}


It was found that the optimal orientation for the neutral gait phase yielded stress values more similar to the intact femur in the overall proximal region when compared to results from the complete DOE design space. However, when examining the individual Regions of Interest, the neutral phase optimal orientation did not necessarily produce optimal regional results when compared to the rest of the design space. This was due to the inherent compromises in the optimal orientation calculations required to achieve maximal desirability, in addition to the compromises caused by the weighted averages for each overall optimal orientation. In the neutral phase, the Superior Region was most similar to the intact femur when the stem was implanted in $4 \mathrm{~mm}$ of inferior positioning, while the Lateral Region developed the lowest stress values under $10^{\circ}$ valgus; both of which are extremes that were not selected for in the optimal orientation calculations. However, while the regional stress values may not be most similar to the intact femur values when compared to the extremes of the design space, on the whole the stress developed in the proximal femur was optimal. This indicates successful optimization of the neutral gait phase DOE.

In the extension gait phase DOE, the Proximal Region was again found to yield stress values most similar to the intact femur when compared to the DOE design space. Similar to the neutral gait phase, the optimal orientation under the extension phase did not yield stress values most analogous to the intact femur across all regions, due to specific regional requirements for optimality. For example the Inferior Region favoured a more anteverted stem with no anterior or posterior positioning, while the Lateral Region yielded optimal stress values under maximal valgus angulation. However, given that the Proximal Region produced stress values most akin to the intact femur, it can again be concluded that overall the extension phase optimal orientation was in fact optimal, and hence the DOE was successful.

Within the flexion gait phase DOE, the optimal orientation FE model generated stress values most comparable to the intact femur on the whole proximally when compared to the entirety of the design space. There were again regional differences that caused specific areas to favour more extreme implant orientations not included in the desirability calculations. The Inferior Region benefited most from a neutrally angulated and positioned stem while the Superior Region favoured a varus and slightly inferior orientation, and the Lateral Region was optimal under maximal valgus angulation. Since the stresses developed in the optimally implanted flexion 
femur were most similar to the intact femur in the Proximal Region when compared to the rest of the design space, the flexion phase DOE was therefore successfully optimized.

\subsection{Validation of Global Optimal Orientation}

The global optimal orientation FE model had no direct DOE with which to compare, given that it was generated using a weighted average of all of the gait phase optimal orientations. It was therefore loaded under the neutral, extension, and flexion gait phase conditions, and the regional stress values were contrasted with those developed in the respective FE models for each gait phase optimal orientation. Under the neutral and extension loading conditions, values across all global Regions were found to differ by an average of $1 \%$ (Equation 4) from the stress output of the neutral and extension optimal orientation FE models, while global values in flexion differed by $4 \%$ from those in the flexion optimal orientation. This high degree of similarity between the individual optimal orientation models and the global model indicates that this global optimal orientation is a good representative of each gait cycle optimal orientation. The global optimal orientation was therefore defined as the optimal orientation of a straight short stem hip implant for the range of the gait cycle. 


\section{CHAPTER 7 \\ Discussion}

Conservative total hip replacements are becoming increasingly popular in the younger, more active population, as they allow for a minimally invasive surgical approach, conserve proximal bone stock, and result in more physiological stress distributions. ${ }^{25,45,46}$ However, there is a lack of biomechanical data to support these implants, in particular data pertaining to the optimal implant angle and position. This study endeavoured to ascertain the optimal orientation for a straight short stem hip implant within the proximal femur, as determined by its stress difference from the intact femur. It was hypothesized that a valgus and inferior orientation would be

optimal, based on previous studies of similarly shaped implants. ${ }^{52-56}$ Through evaluation of the optimal orientations generated using DOE for each gait cycle loading condition, it was found that increasing valgus yields a stress distribution more similar to an intact femur, while greater inferior positioning is only beneficial for the superior neck region.

\subsection{Optimal Orientation}

The optimal orientation for the neutral hip phase during walking was given by parameter settings of $8.9^{\circ}$ valgus, $0^{\circ}$ anteversion, $0.11 \mathrm{~mm}$ anterior position and $0.40 \mathrm{~mm}$ inferior position. The hip extension phase during walking yielded similar values for the optimal orientation, with $7.99^{\circ}$ valgus, $0.27^{\circ}$ anteversion, $0.46 \mathrm{~mm}$ posterior position and $0 \mathrm{~mm}$ inferior position. Finally, the hip flexion phase during walking exhibited a slightly different optimal orientation with $10^{\circ}$ valgus, $10^{\circ}$ anteversion, $0.74 \mathrm{~mm}$ posterior position and $0.41 \mathrm{~mm}$ inferior position. Each of these orientations was seen to minimize the difference from the intact femur in terms of either raw stress values or percent of intact femur values. A global optimal orientation was also determined, and was defined by parameter settings of $9.14^{\circ}$ valgus, $2.49^{\circ}$ anteversion, $0.48 \mathrm{~mm}$ posterior position, and $0.23 \mathrm{~mm}$ inferior position. The stress distribution resulting from an FE model implanted with a stem in the global optimal orientation and loaded under the range of potential gait cycle conditions was found to be very similar to the respective stress distributions resulting from a stem implanted in each gait phase optimal orientation. The global optimal orientation was therefore defined as the optimal orientation for a straight short stem hip implant. 
All of these orientation factor levels fall within optimal ranges for the surgical implantation of hip implants as determined in the literature. It has been found that for femurs with a relatively varus native $\mathrm{CCD}$ angle, placement of the femoral component of hip resurfacings in up to $10^{\circ}$ valgus is optimal. ${ }^{52-54,117}$ According to Yoshimine et al., the clinically relevant range of neck anteversion angles measured from the coronal plane for total hip replacements is between $0-30^{\circ}$ (equivalent SNA range of $0-25^{\circ}$ for the synthetic femur), with a lower anteversion level recommended if anteversion of the acetabular cup is increased. ${ }^{118}$ Higher levels of anteversion have been found to increase the risk for posterior impingement, suggesting lower levels are optimal. ${ }^{119}$ Since implant positioning is governed by both the size of the implant and its anchoring location within the femur, there are very few studies that assess the optimal position of any type of implant. However, a study by Speirs et al. examined the effect of moving an implant into an offset position with $2 \mathrm{~mm}$ of posterior positioning and $4 \mathrm{~mm}$ of superior positioning, and found that the relative changes in proximal cortical strain due to this orientation were negligible when compared to overall changes from the intact femur. ${ }^{62}$ This suggests that for minor changes in position within the proximal femur, the resultant strain distribution is only minimally affected, and hence the small range of implant positions suggested by the optimal orientations would not significantly affect the outcome response.

\subsubsection{Common Significant Terms}

Through analysis of each DOE, it was determined that there were a number of significant terms which appeared in many of the models, regardless of the loading situation. The first such term was factor A, corresponding to the varus/valgus orientation, which commonly exhibited high significance $(p<0.05)$ alongside a large negative coefficient. This indicated that any deviation into the varus (negative) orientation would act to increase the factor's contribution to the outcome response, thereby increasing the difference from the intact femur value. For this reason, none of the optimal orientations included any appreciable varus angulation, and instead tended toward the maximum valgus range. This is in line with previous studies on optimal varus/valgus implant orientation, which determined a valgus position yielded strain values more similar to an intact femur, and furthermore increased the load to failure. ${ }^{52,54,117}$

Another term that was found to be significant $(p<0.05)$ throughout the range of the gait cycle was the two-factor interaction between $\mathrm{B}$ and $\mathrm{C}$, or anteversion and the posterior/anterior position. 
The large positive coefficient arising for this term in many of the models suggests a positive correlation between anteversion and the anterior position. This is not surprising when considering the anatomy of the femoral neck, for which the combination of anteversion and an anterior position would tend to bring the implant closer to the anterior cortex. Any interaction between the implant stem and the cortical bone was noted to greatly increase the local stress, which would have subsequently affected the mean stresses developed in the Inferior, Superior and Proximal Regions.

The second order interaction of factor $\mathrm{C}$ was significant $(p<0.0001)$ throughout the range of the gait cycle, usually occurring alongside a highly positive coefficient. This would have the effect of yielding a positive contribution to the response outcome regardless of the sign of the factor value. Indeed, the response surface for this factor combination usually resembled an inverse quadratic, which suggests that any large deviation from the neutral lateral position would tend to increase the resulting stresses in the proximal femur. In a study by Kuzyk et al. it was found that anterior or posterior positioning of a femoral head lag screw decreased the load to failure, which indicates that such positioning yields a detrimental biomechanical response in the bone. ${ }^{56}$

\subsubsection{Region- and Phase-Specific Significant Terms}

Many of the response outcome models resulted in region-specific significant terms that exhibited a high impact on the stress developed in that particular region. For example factor D, or inferior positioning, was noted to be significant $(p<0.05)$ in many of the response outcome models; however, the sign of its complementary coefficient altered between positive and negative. This was due to the fact that one of the examined regions was the superior neck, for which an increased distance to the implant would result in a correspondingly decreased amount of superior stress propagation. This was supported by the surface profile for this region, which exhibited a linear decrease in the resultant stress or percentage value with increasing inferior positioning. For every other region, the inferior factor had a positive coefficient, indicating a tendency to increase stress in that particular region.

Another factor that regularly displayed a high degree of region-specific impact was factor A, or the varus/valgus angle, within the Lateral Region. It was found that this term tended to have a large negative coefficient, with a high level of significance $(p<0.0001)$. This implies that distal 
loading of the femur is strongly related to the varus/valgus orientation of the stem, with valgus tending to lessen the stress developed in the lateral proximal region.

A number of factors were also noted to gain significance or undergo a coefficient sign reversal depending on the phase of the gait cycle. Factor $\mathrm{C}$ was significant in both the extension and flexion conditions, however in opposite directions. Under extension, factor $\mathrm{C}$ generally had a positive coefficient that indicated higher levels of stress with the anterior position, while the negative coefficient in the flexion condition suggested a posterior position would increase stress. These opposing results are to be expected if one considers the orientation of loading for each condition. When the femur is loaded under extension, its angulation is such that the applied load would tend to result in compression on the anterior aspect of the femur, while flexion would yield a posterior compression. As such, an anterior stem positioning would be disposed toward loading of the anterior cortex under extension, thereby increasing the stress in this region, with a similar effect for posterior positioning in the flexion condition.

It is also interesting to note that factor $\mathrm{B}$, or anteversion, was significant $(p<0.05)$ in the neutral and extension conditions, possessing large positive coefficients that acted to decrease the levels of anteversion recommended by the optimal orientation. Yet under the flexion condition, for which $10^{\circ}$ of anteversion was recommended, it was rarely found to be a significant term. This result can be explained by the positive two-factor interaction of $\mathrm{B}$ with $\mathrm{C}$, which was found to be highly significant in flexion. This interaction suggests that when $\mathrm{C}$ is negative, a positive input for $\mathrm{B}$ is required to minimize the output response. Hence a posterior orientation under flexion must also be combined with increased anteversion, which would act to rotate the stem away from the posterior cortex and thereby aid in minimizing stresses in this region. Another effect contributing to this high level of anteversion was the relatively flat response curve seen in many of the desirability profiles for the flexion condition (Appendix E). This implies that any changes in anteversion would only minimally affect the outcome response, and hence a high or low level of anteversion, as governed by its interaction effects, is irrelevant biomechanically for the flexion condition. 


\subsection{Clinical Relevance}

The global optimal orientation can provide orthopaedic surgeons with a surgical goal when implanting a straight short stem; however, the maximal accuracy that can be expected when using software-guided navigation is only $\pm 1^{\circ}$ and $\pm 1 \mathrm{~mm}$. Since the established global optimal positions are both less than half a millimeter in any direction, it is therefore recommended that surgeons aim to implant the stem in a neutral position. Both the anterior/posterior and inferior positions are relatively sensitive to small positional changes, and thus a neutral position goal would allow for some slight positional inaccuracies without the risk of deviating too far into any one plane. Furthermore, given that the only orientation noted to consistently minimize proximal femur stress was the valgus orientation, it is therefore recommended that this be a primary surgical goal when implanting short stem hip implants. A valgus hip implant angulation is consistent with prior clinical and biomechanical recommendations. ${ }^{52-55,76,117}$ Aside from these recommendations, placement of the stem in any other deviation from neutral should be based upon patient-specific physiology and anatomy. For example, a patient with decreased BMD in the superior proximal region of the femur might benefit from an inferiorly placed stem to minimize the potential for superior neck fracture. Likewise, a patient whose anatomy is such that the anteversion angle is increased could see optimal results from a slightly posterior positioning.

Perhaps the more useful measure for orthopaedic surgeons is the optimality window given by the range of optimal orientation settings for each gait cycle condition (Table 22). While the surgeon should still aim for the global optimal orientation, this optimality window allows for some leeway with regard to stem placement. Since values within this envelope all resulted in optimal orientations, it can be assumed that any factor combination therein would result in minimal changes biomechanically. That said, any orientation that results in contact with the cortical bone will increase the stress developed in that region, and so should be avoided. ${ }^{76}$ Furthermore, given that the high level for the anteversion range was generated by only the flexion condition through an interaction effect, while both of the other conditions experienced detrimental response outcomes directly pertaining to high levels of anteversion, it is therefore recommended that a more neutral version angle be the ultimate surgical goal. 
The present study found the superior and inferior femoral neck to be regions of high stress, which is in accordance with the findings of similar studies. Shah et al. used infrared (IR) thermography to record the principal stress map of an axially loaded synthetic femur, and found the superior femoral neck to be a region of high tensile stress, while the inferior neck exhibited high compressive stresses. ${ }^{86}$ This is further supported by Van Rietbergen et al., who used a micro-finite element model to quantify the strain distribution in healthy and osteoporotic human femurs, and found peak stresses occurred around the femoral neck for both. ${ }^{120}$ Such regions of high stress indicate potential areas of failure, and indeed, fracture along the femoral neck was found to be the most common failure mode in a study by Nicayenzi et al., which axially loaded intact synthetic femurs to failure. ${ }^{116}$ These findings are particularly relevant to the placement of a straight short stem hip implant, as a study by Bishop et al. found that an axially loaded human femur implanted with the Silent Hip most commonly failed due to superior separation of the implant from the cancellous bone, followed by medial cut-out of the femoral neck. ${ }^{103}$ This further supports implanting the femur in a valgus orientation, which acts to align the implant axis with the joint force vector and so reduce the resultant moment, which consequently minimizes the stresses developed in the neck in response to the applied moment. ${ }^{103}$

As with all conservative cementless implants, there are two main reaction stresses that act at the implant-bone interface: the rotation stress caused by the transverse component of the joint force, and the press-fit stress that resists axial subsidence caused by the axial component of the joint force. ${ }^{103}$ These stresses regulate the primary stability achievable in a cementless implant such as the Silent Hip. In addition to the wedging effect generated by the tapered design, the initial press fit of the stem within the proximal cancellous bone introduces radial stresses that provide enough friction to resist relative shear movement. ${ }^{103}$ When the rotational stress is greater than the press fit stress, the contact stresses between the bone and the implant decrease and the implant undergoes separation and subsequent catastrophic failure, as described previously. ${ }^{103} \mathrm{~A}$ more valgus implant angle would therefore act to decrease the moment arm of the transverse component of the joint force and so reduce the rotational stress; ensuring contact stresses at the implant-bone interface remain high. According to Woolson et al., cementless stems that achieve a stable initial press fit can undergo full weight bearing immediately with good results. ${ }^{121}$ However, a parametric analysis of the implanted Silent Hip established that interface stresses developed linearly with the magnitude of the joint load. ${ }^{103}$ Given the high peak joint forces 
developed during fast walking and especially stumbling, which can be greater than $800 \%$ of BW, it is therefore recommended that patients undergo only partial weight-bearing post-operatively, in order to minimize the risk of the rotational stress exceeding the press fit stress. ${ }^{113}$

Orthopaedic surgeons should be aware that implantation of any hip implant in an angle more valgus than the natural CCD angle will tend to change the centre of rotation of the hip joint and increase the patient leg-length. Modifications in the hip centre of rotation may affect the lengths and moment arms of the muscles acting about the hip joint, and so decrease their ability to generate forces and moments adequate for the activities of daily living. ${ }^{122,123}$ Furthermore, leglength discrepancies following THA can give rise to a number of postoperative complications, such as nerve palsy, low-back pain, and abnormal gait. ${ }^{124}$ The Silent Hip is a modular hip implant with both femoral stem and head components. As such, if the implant is placed in the recommended valgus orientation, the surgeon has the option of choosing from four different possible head offsets to account for the modified joint centre and leg-length. ${ }^{76}$ It has been shown that a leg-length discrepancy of $2 \mathrm{~cm}$ or greater has a significant effect on physiological parameters postoperatively, such as oxygen consumption and the rate of perceived exertion. ${ }^{125}$ However, patients can be aware and disturbed by an inequality of only $1 \mathrm{~cm}$, and hence orthopaedic surgeons should aim to minimize any discrepancies. ${ }^{126}$ Given that the present study validated a maximum valgus orientation of up to only $10^{\circ}$ valgus, it is unlikely that even a stem implanted in the maximum potential orientation will yield a leg-length discrepancy of greater than $1 \mathrm{~cm}$. A study evaluating the effect of valgus orientation on fracture load also found that the biomechanical benefits of a valgus orientation may decrease for implant placements of greater than $10^{\circ}$ valgus. ${ }^{52}$ It is therefore recommended that orthopaedic surgeons implant straight short stem hip implants in up to, but not exceeding, $10^{\circ}$ valgus.

\subsection{Study Limitations and Future Directions}

One primary limitation of this study was that the contribution of muscle forces to the loads developed in the proximal femur were not considered. It was therefore not possible to predict the degree to which stress shielding might occur, as resultant stresses were not directly comparable to physiological values. However, this was determined to be a reasonable exclusion for this study, as results were based on minimizing the differences to an identically loaded intact femur. Furthermore, studies have shown that inclusion of muscle forces does not significantly affect the 
stress distribution in the proximal metaphysis. ${ }^{127,128}$ Future work might include developing and validating a muscle model to examine the magnitude and localization of stresses under a physiologically loaded femur.

Another limitation of this study was the use of synthetic femurs instead of human cadaver bones, as synthetic bones are less able to mimic the biological response of human bone. Specifically, cancellous architecture in human femurs follows lines of applied stress, whereas cancellous material in synthetic femurs is isotropic and homogeneous. Moreover, no commercially-available synthetic femurs exist on the market that replicate the thinning of human femoral cortical bone during osteoporosis. However, due to the interspecimen variability introduced by cadaver bones, many comparison studies have determined that the physiological benefits of using human bones were outweighed by the disadvantage of variable specimens, which would negatively affect the results. ${ }^{79,83-85,97}$ Synthetic femurs have furthermore been experimentally validated against human femurs and have been used successfully in prior biomechanical studies. ${ }^{56,65,129,130}$

It should be recognized that the femurs in this study were loaded quasistatically instead of in a cyclic or dynamic manner, which would be more representative of physiological loading conditions. ${ }^{113,131}$ Cyclic loading would additionally provide information as to the fatigue failure of the implant, and stiffness changes over time. ${ }^{131}$ However, the goal of this study was only to compare the relative stress distributions between implant orientations, the relationship between which should be constant over time, and hence quasistatic loading conditions were deemed sufficient. ${ }^{83}$ Furthermore, this loading mode facilitated use of FEA, which is commonly validated against quasistatic results. ${ }^{52,65,83,93,97}$

Experimental protocol did not require loading of the femurs to failure, which is frequently completed in evaluations of fracture fixation devices to determine their behaviours under failure conditions. ${ }^{91,92,131}$ In this study, subclinical loading was used predominantly because the main goal of the experimental tests was to validate the FE model. However, loading femurs until catastrophic structural failure occurs is useful in determining failure modes, and assessing peak failure forces and displacements. ${ }^{116}$ For that reason, future studies could include loading the optimal implant orientations for each gait phase to failure, allowing for evaluation of their behaviours under failure conditions. 
Furthermore, such future fracture models could evaluate the effect of strain rate on the apparent stiffness and ultimate fracture load of implanted femurs. Strain rate is relevant both under cyclic loading conditions, and when considering loading conditions representative of a fall. ${ }^{132,133}$ The strain rate during normal activities such as walking and jogging is typically too low to cause bone damage in and of itself; however, cyclic loading can lead to the development of microdamage that acts to decrease the apparent strength and modulus of bone, potentially leading to failure. ${ }^{134}$ Under fall conditions, the strain rate is significantly higher and so engages the viscoelastic nature of bone, acting to both increase the stiffness and the load to failure of the femur. ${ }^{132}$ Future failure models of the implanted stem could therefore take into account the viscoelastic nature of bone under varying strain rates by assessing both low-level cyclic loading of the femur and high strain rate loading conditions.

Another experimental drawback was the use of a flat loading plate instead of an acetabular-like loading cup with a polymer liner, which simulates the hip joint in a THA. ${ }^{79,83,93,101}$ Firstly, a polymer liner was not used to ensure that the true stiffness of the femur-implant construct could be measured without the influence of a soft polymer. ${ }^{101}$ With regard to the loading plate, it can prove difficult to ensure consistent alignment of the femur within the Instron machine when using a loading cup, and thus lateral loads might be transmitted under some loading scenarios but not others. It was therefore determined that for the subclinical loads employed in this study, a flat loading plate would suffice, and would eliminate the potential for any lateral load transmission that might not be accounted for in the FE model. Furthermore, flat loading plates are often used in the literature with good results. ${ }^{85,91,92,94}$ It is not expected that use of a loading cup would significantly modify the optimally determined orientations, which were based on relative stress values. However, it might affect the actual stress distribution within the femur, and thus should be considered in future studies to allow for direct comparison to clinical results.

Finally, only axial compression was considered, and so this study did not evaluate the strain distribution under lateral or torsional loading. Axial compression is the primary loading mechanism in the hip joint, and it also gives rise to higher strain values than either lateral or torsional loading. ${ }^{135}$ Axial loading is therefore the most relevant loading mechanism when evaluating stress distributions that would affect long-term stress shielding, as with the current study. Furthermore, axial loading is one of the most widespread loading mechanisms seen in the literature, and hence there is good precedent for using this loading mode. ${ }^{85,105,116,136}$ However, 
given that fractures due to falls are commonly seen in clinical cases, it may therefore be beneficial for future studies to examine the behaviour of the femur under loading conditions representative of a fall. ${ }^{88,137,138}$

For FEA, bonded contact was assigned between the stem and the cancellous bone, and the joint load was applied directly to the femoral head. In actuality, contact between the implant and the cancellous bone was not bonded under experimental conditions, and load was applied to the superior femoral head through a flat loading plate. Contact at both of these regions would have been most similar to a frictional condition, as has been done in many previous studies. ${ }^{139-141}$ However, frictional contact is a nonlinear contact that would act to greatly increase the computational time, and also requires knowledge of the specific friction coefficients throughout the model. Bonded contact is therefore a more common assumption in FE modelling, in particular when the goal is to simulate full bony osseointegration with the implant. ${ }^{83,90,93}$ It has furthermore been shown that motion at the stem-bone interface is minimal during static axial loading. ${ }^{79}$ Given the number of models required for DOE and the static loading condition, bonded contact was therefore considered to be appropriate for this study. However, this assumption also has implications for the stiffness of the model, as it would have acted to increase the FE model stiffness. Further work could therefore include experimental analyses to determine adequate friction coefficients at the implant-bone interface and between the superior femoral head and the flat loading plate, for eventual use of frictional contact at these regions. If the computational time of such a model is determined to be too great, then bonded contact could be used along with an intermediary stiffness layer at the implant-bone interface. This is a particular property of the studied Silent stem, as this region would actually be composed of both the sintered titanium beads and bone, which would therefore yield a boundary layer containing the averaged stiffness properties of both. ${ }^{68}$

The slope of the validation graph for the FE model was 1.18 instead of 1 (Figure 62). This suggests that the FE model was stiffer than the experimental implant-femur constructs, as the strains experienced by the FE model were lower than experimental strains. This could have been due to a number of reasons, including micromotions between the implant-bone interface and bone-cement cube interface that were not accounted for in the FE model, both of which could have led to a reduction in the stiffness of the experimental femurs. The synthetic femur was also modelled as a linear elastic material with isotropic behaviour for FEA, which is not truly 
representative of synthetic femurs, as they possess both longitudinal and transverse elastic moduli. ${ }^{115}$ Furthermore, the boundary conditions used to simulate experimental conditions were approximations only, in particular the direct application of force versus a more realistic frictional contact with a flat loading plate. However, given the high value of the correlation coefficient in the validation graph, it can be concluded that the FE model produced consistently lower strain values, and thus a correction factor could have been applied to generate a validation graph with a slope of 1 . Since the study was comparative in nature, with various potential implant orientations directly compared, the multiplication of their stresses by the same constant would not have altered the relative trends observed and final conclusions reached. 


\section{CHAPTER 8 \\ Conclusions}

This study endeavoured to determine the optimal orientation of a straight short stem hip implant

within the proximal femur for improved physiological stress distribution. A global optimal orientation of $9.14^{\circ}$ valgus, $2.49^{\circ}$ anteversion, $0.48 \mathrm{~mm}$ posterior position, and $0.23 \mathrm{~mm}$ inferior position was found to yield stress distributions most similar to the intact femur across the range of the gait cycle. In general, it was determined that the valgus orientation was optimal throughout the range of the gait cycle, consistently exhibiting a stress distribution more similar to that of the intact femur. Minimal levels of anterior/posterior and inferior positioning were seen to be beneficial in achieving more physiological stresses in specific regions of interest within the proximal femur, while the anteverted orientation was only beneficial in loading under flexion. Overall, orthopaedic surgeons should aim to implant the stem in valgus up to $10^{\circ}$, with an otherwise neutral position and version, unless some degree of deviation would be beneficial for a patient-specific reason. 


\section{References}

1. Morgan, E.F. and Bouxsein, M.L., Biomechanics of Bone and Age-Related Fractures, in Principles of Bone Biology, Two-Volume Set, J.P. Bilezikian, L.G. Raisz, and T.J. Martin, Editors. 2008, Academic Press: San Diego. p. 29-46.

2. Ontañón, M., Aparicio, C., Ginebra, M.P., and Planell, J.A., Structure and Mechanical Properties of Cortical Bone, in Structural Biological Materials: Design and StructureProperty Relationships, M. Elices, Editor. 2000, Elsevier: Kidlington, Oxford. p. 33-71.

3. Asgharpour, Z., Zioupos, P., Graw, M., and Peldschus, S., Development of a strain rate dependent material model of human cortical bone for computer-aided reconstruction of injury mechanisms. Forensic science international, 2014. 236: p. 109-116.

4. Schaffler, M., Radin, E., and Burr, D., Mechanical and morphological effects of strain rate on fatigue of compact bone. Bone, 1989. 10(3): p. 207-214.

5. McElhaney, J.H., Dynamic response of bone and muscle tissue. J. appl. Physiol, 1966. 21(4): p. 1231-1236.

6. Dong, X.N., Luo, Q., Sparkman, D.M., Millwater, H.R., and Wang, X., Random field assessment of nanoscopic inhomogeneity of bone. Bone, 2010. 47(6): p. 1080-1084.

7. Mow, V.C. and Huiskes, R., Basic Orthopaedic Biomechanics \& Mechano-Biology. 3rd ed. Vol. 3. 2005, Philadelphia, PA, USA: Lippincott Williams \& Wilkins.

8. Knight, S.R., Aujla, R., and Biswas, S.P., Total Hip Arthroplasty-over 100 years of operative history. Orthopedic reviews, 2011. 3(2): p. e16-e16.

9. Bijlsma, J.W.J., Berenbaum, F., and Lafeber, F.P.J.G., Osteoarthritis: an update with relevance for clinical practice. The Lancet, 2011. 377(9783): p. 2115-2126.

10. NIH Osteoporosis and Related Bone Diseases National Resource Center Osteoporosis and Arthritis: Two Common but Different Conditions. January 2012.

11. Canadian Institute for Health Information, Hip and Knee Replacements in Canada: Canadian Joint Replacement Registry 2013 Annual Report. 2013: Toronto.

12. American Academy of Orthopaedic Surgeons. Total Hip Replacement. December 2011 December 2011 [cited 2014 June 10]; Available from: http://orthoinfo.aaos.org/topic.cfm?topic=a00377.

13. NIH National Institute of Arthritis and Musculoskeletal and Skin Diseases. Questions and Answers about Hip Replacement. July 2013 [cited 2014 June 10]; Available from: http://www.niams.nih.gov/Health_Info/Hip_Replacement/default.asp\#foot1.

14. NIH Senior Health. Who Needs a Hip Replacement? [cited 2014 June 10]; Available from: http://nihseniorhealth.gov/hipreplacement/whoneeds/01.html. 
15. Badley, E.M. and Wang, P.P., Arthritis and the aging population: projections of arthritis prevalence in Canada 1991 to 2031. The Journal of rheumatology, 1998. 25(1): p. 138144.

16. American Academy of Orthopaedic Surgeons. Joint Revision Surgery - When Do I Need It? 2007; Available from: http://orthoinfo.aaos.org/topic.cfm?topic=A00510.

17. DePuy International Ltd., Silent Performs Naturally: Product Rationale. 2010.

18. Paprosky, W.G., Greidanus, N.V., and Antoniou, J., Minimum 10-year-results of extensively porous-coated stems in revision hip arthroplasty. Clinical orthopaedics and related research, 1999. 369: p. 230-242.

19. Kluge, W.H., (vii) Current developments in short stem femoral implants for hip replacement surgery. Orthopaedics and Trauma, 2009. 23(1): p. 46-51.

20. Valverde-Mordt, C. and Valverde-Belda, D., Conservative femoral implants. Short stems. Revista Española de Cirugía Ortopédica y Traumatología (English Edition), 2012. 56(1): p. $72-79$.

21. Corten, K., Bourne, R.B., Charron, K.D., Au, K., and Rorabeck, C.H., What works best, a cemented or cementless primary total hip arthroplasty?: minimum 17-year followup of a randomized controlled trial. Clinical Orthopaedics and Related Research®, 2011. 469(1): p. 209-217.

22. Duffy, G.P., Berry, D.J., Rowland, C., and Cabanela, M.E., Primary uncemented total hip arthroplasty in patients $<40$ years old: 10-to 14-year results using first-generation proximally porous-coated implants. The Journal of arthroplasty, 2001. 16(8): p. 140-144.

23. Dorr, L.D., Kane Iii, T.J., and Conaty, J.P., Long-term results of cemented total hip arthroplasty in patients 45 years old or younger: A 16-year follow-up study. The Journal of Arthroplasty, 1994. 9(5): p. 453-456.

24. Morshed, S., Bozic, K.J., Ries, M.D., Malchau, H., and Colford Jr, J.M., Comparison of cemented and uncemented fixation in total hip replacement: a meta-analysis. Acta orthopaedica, 2007. 78(3): p. 315-326.

25. Santori, N., Lucidi, M., and Santori, F.S., Proximal load transfer with a stemless uncemented femoral implant. Journal of Orthopaedics and Traumatology, 2006. 7(3): p. 154-160.

26. Fetto, J., Leali, A., and Moroz, A., Evolution of the Koch model of the biomechanics of the hip: clinical perspective. Journal of orthopaedic science, 2002. 7(6): p. 724-730.

27. Wolff, J., Maquet, P., and Furlong, R., The law of bone remodelling. 1986: Springer Berlin. 
28. Huiskes, R., Weinans, H., and Van Rietbergen, B., The relationship between stress shielding and bone resorption around total hip stems and the effects of flexible materials. Clinical Orthopaedics and Related Research, 1992. 274: p. 124-134.

29. Kim, Y.H. and Kim, Y.H., The results of a proximally-coated cementless femoral component in total hip replacement: A FIVE-TO 12-YEAR FOLLOW-UP. Journal of Bone and Joint Surgery - British Volume, 2008. 90-B(3): p. 299-305.

30. Sumner, D., Turner, T., Igloria, R., Urban, R., and Galante, J., Functional adaptation and ingrowth of bone vary as a function of hip implant stiffness. Journal of biomechanics, 1998. 31(10): p. 909-917.

31. Decking, R., Puhl, W., Simon, U., and Claes, L.E., Changes in strain distribution of loaded proximal femora caused by different types of cementless femoral stems. Clinical Biomechanics, 2006. 21(5): p. 495-501.

32. Glassman, A., Bobyn, J., and Tanzer, M., New femoral designs: do they influence stress shielding? Clinical orthopaedics and related research, 2006. 453: p. 64-74.

33. Nishino, T., Mishima, H., Miyakawa, S., Kawamura, H., and Ochiai, N., Midterm results of the Synergy cementless tapered stem: stress shielding and bone quality. Journal of Orthopaedic Science, 2008. 13(6): p. 498-503.

34. Engh, C., Bobyn, J., and Glassman, A., Porous-coated hip replacement. The factors governing bone ingrowth, stress shielding, and clinical results. Journal of Bone \& Joint Surgery, British Volume, 1987. 69(1): p. 45-55.

35. Kawate, K., Ohneda, Y., Ohmura, T., Yajima, H., Sugimoto, K., and Takakura, Y., Computed tomography-based custom-made stem for dysplastic hips in japanese patients. The Journal of arthroplasty, 2009. 24(1): p. 65-70.

36. Bieger, R., Ignatius, A., Reichel, H., and Dürselen, L., Biomechanics of a short stem: In vitro primary stability and stress shielding of a conservative cementless hip stem. Journal of Orthopaedic Research, 2013. 31(8): p. 1180-1186.

37. Chen, H.-H., Morrey, B.F., An, K.-N., and Luo, Z.-P., Bone remodeling characteristics of a short-stemmed total hip replacement. The Journal of arthroplasty, 2009. 24(6): p. 945950.

38. Gruen, T.A., McNeice, G.M., and Amstutz, H.C., "Modes of failure" of cemented stemtype femoral components: a radiographic analysis of loosening. Clinical Orthopaedics and Related Research, 1979. 141: p. 17-27.

39. McCaskie, A., Brown, A., Thompson, J., and Gregg, P., Radiological Evaluation of the Interfaces After Cemented Total Hip Replacement: Interobserver and Intraobserver Agreement. Journal of Bone \& Joint Surgery, British Volume, 1996. 78(2): p. 191-194.

40. Tóth, K., Mécs, L., and Kellermann, P., Early experience with the DePuy Proxima ${ }^{\mathrm{TM}}$ short stem in total hip arthroplasty. Acta Orthopædica Belgica, 2010. 76(5): p. 613. 
41. Carlsson, L.V., Albrektsson, T., Albrektsson, B.E., Jacobsson, C.M., Macdonald, W., Regnér, L., and Weidenhielm, L.R., Stepwise introduction of a bone-conserving osseointegrated hip arthroplasty using RSA and a randomized study: II. Clinical proof of concept-40 patients followed for 2 years. Acta orthopaedica, 2006. 77(4): p. 559-566.

42. Gronewold, J., Berner, S., Olender, G., Hurschler, C., Windhagen, H., von Lewinski, G., and Floerkemeier, T., Changes in strain patterns after implantation of a short stem with metaphyseal anchorage compared to a standard stem: an experimental study in synthetic bone. Orthopedic reviews, 2014. 6(1).

43. Santori, F. and Santori, N., Mid-term results of a custom-made short proximal loading femoral component. Journal of Bone \& Joint Surgery, British Volume, 2010. 92(9): p. 1231-1237.

44. Oldenrijk, J.v., Molleman, J., Klaver, M., Poolman, R.W., and Haverkamp, D., Revision rate after short-stem total hip arthroplasty: A systematic review of 49 clinical studies. Acta orthopaedica, 2014. 85(3): p. 1-9.

45. Learmonth, I.D., (ii) Conservative hip implants. Current Orthopaedics, 2005. 19(4): p. 255-262.

46. Briem, D., Schneider, M., Bogner, N., Botha, N., Gebauer, M., Gehrke, T., and Schwantes, B., Mid-term results of 155 patients treated with a collum femoris preserving (CFP) short stem prosthesis. International Orthopaedics, 2011. 35(5): p. 655-660.

47. Dabirrahmani, D., Hogg, M., Kohan, L., and Gillies, M., Primary and long-term stability of a short-stem hip implant. Proceedings of the Institution of Mechanical Engineers, Part H: Journal of Engineering in Medicine, 2010. 224(9): p. 1109-1119.

48. Hutt, J., Harb, Z., Gill, I., Kashif, F., Miller, J., and Dodd, M., Ten year results of the collum femoris preserving total hip replacement: a prospective cohort study of seventy five patients. International Orthopaedics, 2013: p. 1-6.

49. Malhotra, R., Mastering Orthopaedic Techniques - Total Hip Arthroplasty, R. Malhotra and V. Kumar, Editors. 2012, Jaypee Brothers Medical Publishers (P) Ltd. p. 241 - 254.

50. Maheson, M., Honl, M., Sullivan, J., Kurth, A., Pace, N., Piriou, P., Verheul, R., and Waller, C., The Silent ${ }^{\mathrm{TM}}$ Hip - A new solution in primary total hip arthroplasty. Short- to mid-term results from two clinical studies across eight centres. BOA 2009 Poster.

51. Waller, C.S. and McTighe, T., An Alternative Conservative Approach to Hip Reconstruction. Joint Implant Surgery and Research Foundation Reconstructive Review, 2013. 3(2): p. 20-27.

52. Anglin, C., Masri, B.A., Tonetti, J., Hodgson, A.J., and Greidanus, N.V., Hip resurfacing femoral neck fracture influenced by valgus placement. Clinical orthopaedics and related research, 2007. 465: p. 71-79. 
53. Davis, E., Olsen, M., Zdero, R., Waddell, J., and Schemitsch, E., Femoral neck fracture following hip resurfacing THE EFFECT OF ALIGNMENT OF THE FEMORAL COMPONENT. Journal of Bone \& Joint Surgery, British Volume, 2008. 90(11): p. 15221527.

54. Radcliffe, I. and Taylor, M., Investigation into the effect of varus-valgus orientation on load transfer in the resurfaced femoral head: A multi-femur finite element analysis. Clinical Biomechanics, 2007. 22(7): p. 780-786.

55. Olsen, M., Lewis, P.M., Waddell, J.P., and Schemitsch, E.H., A biomechanical investigation of implant alignment and femoral neck notching with the Birmingham MidHead Resection. The Journal of arthroplasty, 2010. 25(6): p. 112-117.

56. Kuzyk, P.R., Zdero, R., Shah, S., Olsen, M., Waddell, J.P., and Schemitsch, E.H., Femoral head lag screw position for cephalomedullary nails: a biomechanical analysis. Journal of orthopaedic trauma, 2012. 26(7): p. 414-421.

57. McCalden, R., Naudie, D., Thompson, A., and Moore, C., RSA analysis of early migration of the uncemented SMFTM vs SYNERGYTM stem: A prospective randomized controlled trial. Bone \& Joint Science, 2011. 1(2).

58. McMinn, D. The Birmingham Mid Head Resection. [cited 2013 January 25]; Available from: http://www.mcminncentre.co.uk/birmingham-mid-head-resection.html.

59. Choy, G.G.H., Roe, J.A., Whitehouse, S.L., Cashman, K.S., and Crawford, R.W., Exeter Short Stems Compared With Standard Length Exeter Stems: Experience From the Australian Orthopaedic Association National Joint Replacement Registry. The Journal of Arthroplasty, 2013. 28(1): p. 103-109.e1.

60. Xing, Z., Moon, B., Satcher, R., Lin, P., and Lewis, V., A Long Femoral Stem Is Not Always Required in Hip Arthroplasty for Patients With Proximal Femur Metastases. Clinical Orthopaedics and Related Research®, 2013. 471(5): p. 1622-1627.

61. Van Rietbergen, B. and Huiskes, R., Load transfer and stress shielding of the hydroxyapatite-ABG hip: a study of stem length and proximal fixation. The Journal of arthroplasty, 2001. 16(8): p. 55-63.

62. Speirs, A.D., Heller, M.O., Taylor, W.R., Duda, G.N., and Perka, C., Influence of changes in stem positioning on femoral loading after THR using a short-stemmed hip implant. Clinical Biomechanics, 2007. 22(4): p. 431-439.

63. Sawbones Worldwide. Composite Bones. 2013 [cited 2013 April 5]; Available from: http://www.sawbones.com/Catalog/Biomechanical/Composite\%20Bones/3406.

64. Cristofolini, L., Viceconti, M., Cappello, A., and Toni, A., Mechanical validation of whole bone composite femur models. Journal of biomechanics, 1996. 29(4): p. 525-535.

65. Papini, M., Zdero, R., Schemitsch, E., and Zalzal, P., The biomechanics of human femurs in axial and torsional loading: comparison of finite element analysis, human cadaveric 
femurs, and synthetic femurs. Journal of biomechanical engineering, 2007. 129(1): p. 1219.

66. Davies, J.E., The Bone-Biomaterial Interface. 1991, Toronto: University of Toronto Press.

67. Zimmer. Hip: Ceramic-on-Ceramic - Scientific Information. 2013 [cited 2014 May 23]; Available from: http://www.zimmer.com/en-ZA/hcp/hip/our-science/ceramic-onceramic.jspx\#1Reference.

68. Brooks, J., Principal Mechanical Design Engineer - Hips at DePuy Synthes, G.E. Cook, Editor. February 21, 2014.

69. Heros, R.J., Senior Scientific \& Sales Consultant at CeramTec Medical Products, G.E. Cook, Editor. February 5, 2014.

70. Heros, R.J., Validation of a New High Performance Alumina Matrix Composite for use in Total Joint Replacement. CeramTec Medical Products.

71. Schemitsch, E.H., Head Division Orthopaedic Surgery, St. Michael's Hospital, G.E. Cook, Editor. 2013: Toronto, ON.

72. Ganapathi, M., Vendittoli, P.-A., Lavigne, M., and Günther, K.-P., Femoral component positioning in hip resurfacing with and without navigation. Clinical orthopaedics and related research, 2009. 467(5): p. 1341-1347.

73. Olsen, M., Chiu, M., Gamble, P., Boyle, R.A., Tumia, N., and Schemitsch, E.H., A comparison of conventional guidewire alignment jigs with imageless computer navigation in hip resurfacing arthroplasty. The Journal of Bone \& Joint Surgery, 2010. 92(9): p. 1834-1841.

74. Davis, E., Gallie, P., Macgroarty, K., Waddell, J., and Schemitsch, E., The accuracy of image-free computer navigation in the placement of the femoral component of the Birmingham Hip Resurfacing A CADAVER STUDY. Journal of Bone \& Joint Surgery, British Volume, 2007. 89(4): p. 557-560.

75. McMinn, D. The Birmingham Hip Resurfacing. [cited 2014 June 15]; Available from: http://www.mominncentre.co.uk/birmingham-hip-resurfacing.html.

76. DePuy International Ltd., Silent Performs Naturally: Surgical Technique. 2010.

77. Resubal, J.R.E. and Morgan, D.A., Computer-assisted vs conventional mechanical jig technique in hip resurfacing arthroplasty. The Journal of arthroplasty, 2009. 24(3): p. 341-350.

78. Morison, Z., Hip Resurfacing Arthroplasty: Investigating the Femoral Component in the Sagittal Plane, in Institute of Medical Science. 2011, University of Toronto. 
79. Davis, E.T., Olsen, M., Papini, M., Waddell, J.P., Schemitsch, E.H., and Zdero, R., A biomechanical and finite element analysis of femoral neck notching during hip resurfacing. Journal of biomechanical engineering, 2009. 131(4): p. 041002.

80. Kadaba, M., Ramakrishnan, H., Wootten, M., Gainey, J., Gorton, G., and Cochran, G., Repeatability of kinematic, kinetic, and electromyographic data in normal adult gait. Journal of Orthopaedic Research, 1989. 7(6): p. 849-860.

81. Ramakrishnan, H. and Kadaba, M., On the estimation of joint kinematics during gait. Journal of Biomechanics, 1991. 24(10): p. 969-977.

82. Morison, Z., Olsen, M., Higgins, G.A., Zdero, R., and Schemitsch, E.H., The Biomechanical Effect of Notch Size, Notch Location, and Femur Orientation on Hip Resurfacing Failure. 2013.

83. Ebrahimi, H., Rabinovich, M., Vuleta, V., Zalcman, D., Shah, S., Dubov, A., Roy, K., Siddiqui, F.S., Schemitsch, E.H., Bougherara, H., and Zdero, R., Biomechanical properties of an intact, injured, repaired, and healed femur: An experimental and computational study. Journal of the mechanical behavior of biomedical materials, 2012. 16: p. 121-135.

84. Shah, S., Kim, S., Dubov, A., Schemitsch, E., Bougherara, H., and Zdero, R., The biomechanics of plate fixation of periprosthetic femoral fractures near the tip of a total hip implant: cables, screws, or both? Proceedings of the Institution of Mechanical Engineers, Part H: Journal of Engineering in Medicine, 2011. 225(9): p. 845-856.

85. Bougherara, H., Zdero, R., Miric, M., Shah, S., Hardisty, M., Zalzal, P., and Schemitsch, E., The biomechanics of the T2 femoral nailing system: a comparison of synthetic femurs with finite element analysis. Proceedings of the Institution of Mechanical Engineers, Part H: Journal of Engineering in Medicine, 2009. 223(3): p. 303-314.

86. Shah, S., Bougherara, H., Schemitsch, E.H., and Zdero, R., Biomechanical stress maps of an artificial femur obtained using a new infrared thermography technique validated by strain gages. Medical engineering \& physics, 2012. 34(10): p. 1496-1502.

87. Vishay Precision Group, Strain Gage Applications with M-Bond AE-10, AE-15 and GA-2 Adhesive Systems. March 29, 2010, InterTechnology Inc.: Toronto, ON.

88. Lotz, J.C., Cheal, E.J., and Hayes, W.C., Stress distributions within the proximal femur during gait and falls: Implications for osteoporotic fracture. Osteoporosis International, 1995. 5(4): p. 252-261.

89. Grasso, R., Bianchi, L., and Lacquaniti, F., Motor patterns for human gait: backward versus forward locomotion. Journal of Neurophysiology, 1998. 80(4): p. 1868-1885.

90. Bougherara, H., Zdero, R., Shah, S., Miric, M., Papini, M., Zalzal, P., and Schemitsch, E.H., A biomechanical assessment of modular and monoblock revision hip implants using FE analysis and strain gage measurements. Journal of Orthopaedic Surgery and Research, 2010. 5(34). 
91. Dennis, M.G., Simon, J.A., Kummer, F.J., Koval, K.J., and Di Cesare, P.E., Fixation of periprosthetic femoral shaft fractures: a biomechanical comparison of two techniques. Journal of orthopaedic trauma, 2001. 15(3): p. 177-180.

92. Fulkerson, E., Koval, K., Preston, C.F., Iesaka, K., Kummer, F.J., and Egol, K.A., Fixation of periprosthetic femoral shaft fractures associated with cemented femoral stems: a biomechanical comparison of locked plating and conventional cable plates. Journal of orthopaedic trauma, 2006. 20(2): p. 89-93.

93. Dubov, A., Kim, S., Shah, S., Schemitsch, E., Zdero, R., and Bougherara, H., The biomechanics of plate repair of periprosthetic femur fractures near the tip of a total hip implant: the effect of cable-screw position. Proceedings of the Institution of Mechanical Engineers, Part H: Journal of Engineering in Medicine, 2011. 225(9): p. 857-865.

94. Taddei, F., Cristofolini, L., Martelli, S., Gill, H.S., and Viceconti, M., Subject-specific finite element models of long bones: An in vitro evaluation of the overall accuracy. Journal of Biomechanics, 2006. 39(13): p. 2457-2467.

95. Department of Aerospace Engineering Sciences, FEM Modeling: Introduction, in Introduction to Finite Element Methods. September 8, 2013, University of Colorado at Boulder. p. 1-15.

96. ANSYS Inc., ANSYS Workbench - Mechanical Introdution, in Training Manual. 2009, ANSYS, Inc. Proprietary.

97. Cheung, G., Zalzal, P., Bhandari, M., Spelt, J., and Papini, M., Finite element analysis of a femoral retrograde intramedullary nail subject to gait loading. Medical engineering \& physics, 2004. 26(2): p. 93-108.

98. Mirzaei, M., Samiezadeh, S., Khodadadi, A., and Ghazavi, M.R., Finite Element Prediction and Experimental Verification of the Failure Pattern of Proximal Femur using Quantitative Computed Tomography Images. World Academy of Science, Engineering and Technology, 2012. 66: p. 104-111.

99. McNamara, B.P., Cristofolini, L., Toni, A., and Taylor, D., Relationship between boneprosthesis bonding and load transfer in total hip reconstruction. Journal of biomechanics, 1997. 30(6): p. 621-630.

100. Wang, C., Yettram, A., Yao, M., and Procter, P., Finite element analysis of a Gamma nail within a fractured femur. Medical engineering \& physics, 1998. 20(9): p. 677-683.

101. Bougherara, H., Zdero, R., Dubov, A., Shah, S., Khurshid, S., and Schemitsch, E.H., A preliminary biomechanical study of a novel carbon-fibre hip implant versus standard metallic hip implants. Medical engineering \& physics, 2011. 33(1): p. 121-128.

102. Viceconti, M., Bellingeri, L., Cristofolini, L., and Toni, A., A comparative study on different methods of automatic mesh generation of human femurs. Medical engineering \& physics, 1998. 20(1): p. 1-10. 
103. Bishop, N.E., Burton, A., Maheson, M., and Morlock, M.M., Biomechanics of short hip endoprostheses - The risk of bone failure increases with decreasing implant size. Clinical Biomechanics, 2010. 25(7): p. 666-674.

104. Cristofolini, L., Juszczyk, M., Taddei, F., and Viceconti, M., Strain distribution in the proximal human femoral metaphysis. Proceedings of the Institution of Mechanical Engineers, Part H: Journal of Engineering in Medicine, 2009. 223(3): p. 273-288.

105. Cristofolini, L., Juszczyk, M., Taddei, F., Field, R., Rushton, N., and Viceconti, M., Stress shielding and stress concentration of contemporary epiphyseal hip prostheses. Proceedings of the Institution of Mechanical Engineers, Part H: Journal of Engineering in Medicine, 2009. 223(1): p. 27-44.

106. Cheal, E.J., Spector, M., and Hayes, W.C., Role of loads and prosthesis material properties on the mechanics of the proximal femur after total hip arthroplasty. Journal of orthopaedic research, 1992. 10(3): p. 405-422.

107. Anderson, M.J. and Whitcomb, P.J., RSM simplified: optimizing processes using response surface methods for design of experiments. 2005: Productivity Press.

108. Anderson, M.J. and Whitcomb, P.J., DOE simplified: practical tools for effective experimentation. 2000: Productivity Portland.

109. NIST/SEMATECH, e-Handbook of Statistical Methods. 2013.

110. Andrei, A., Welkenhuysen, M., Nuttin, B., and Eberle, W., A response surface model predicting the in vivo insertion behavior of micromachined neural implants. Journal of Neural Engineering, 2012. 9(1): p. 1-12.

111. JMP A Business Unit of SAS, JMP Statistics and Graphics Guide, Release 7. 2007, SAS Institute Inc.: Cary, NC, USA.

112. Arjmand, N., Plamondon, A., Shirazi-Adl, A., Larivière, C., and Parnianpour, M., Predictive equations to estimate spinal loads in symmetric lifting tasks. Journal of biomechanics, 2011. 44(1): p. 84-91.

113. Bergmann, G., Deuretzbacher, G., Heller, M., Graichen, F., Rohlmann, A., Strauss, J., and Duda, G., Hip contact forces and gait patterns from routine activities. Journal of biomechanics, 2001. 34(7): p. 859-871.

114. Lengsfeld, M., Schmitt, J., Alter, P., Kaminsky, J., and Leppek, R., Comparison of geometry-based and CT voxel-based finite element modelling and experimental validation. Medical engineering \& physics, 1998. 20(7): p. 515-522.

115. Keyak, J., Fourkas, M., Meagher, J., and Skinner, H., Validation of an automated method of three-dimensional finite element modelling of bone. Journal of Biomedical Engineering, 1993. 15(6): p. 505-509. 
116. Nicayenzi, B., Shah, S., Schemitsch, E., Bougherara, H., and Zdero, R., The biomechanical effect of changes in cancellous bone density on synthetic femur behaviour. Proceedings of the Institution of Mechanical Engineers, Part H: Journal of Engineering in Medicine, 2011. 225(11): p. 1050-1060.

117. Nabavi, A., Yeoh, K., Shidiac, L., Appleyard, R., Gillies, R., and Turnbull, A., Effects of positioning and notching of resurfaced femurs on femoral neck strength: a biomechanical test. Journal of orthopaedic surgery, 2009. 17(1).

118. Yoshimine, F., The safe-zones for combined cup and neck anteversions that fulfill the essential range of motion and their optimum combination in total hip replacements. Journal of biomechanics, 2006. 39(7): p. 1315-1323.

119. Langton, D., Jameson, S., Joyce, T., Hallab, N., Natu, S., and Nargol, A., Early failure of metal-on-metal bearings in hip resurfacing and large-diameter total hip replacement $A$ CONSEQUENCE OF EXCESS WEAR. Journal of Bone \& Joint Surgery, British Volume, 2010. 92(1): p. 38-46.

120. Van Rietbergen, B., Huiskes, R., Eckstein, F., and Rüegsegger, P., Trabecular bone tissue strains in the healthy and osteoporotic human femur. Journal of Bone and Mineral Research, 2003. 18(10): p. 1781-1788.

121. Woolson, S.T. and Adler, N.S., The effect of partial or full weight bearing ambulation after cementless total hip arthroplasty. The Journal of arthroplasty, 2002. 17(7): p. 820825 .

122. Delp, S.L., Komattu, A.V., and Wixson, R.L., Superior displacement of the hip in total joint replacement: Effects of prosthetic neck length, neck-stem angle, and anteversion angle on the moment-generating capacity of the muscles. Journal of orthopaedic research, 1994. 12(6): p. 860-870.

123. Vasavada, A.N., Delp, S.L., Maloney, W.J., Schurman, D.J., and Zajac, F.E., Compensating for changes in muscle length in total hip arthroplasty: effects on the moment generating capacity of the muscles. Clinical orthopaedics and related research, 1994. 302: p. 121-133.

124. Clark, C.R., Huddleston, H.D., Schoch, E.P., and Thomas, B.J., Leg-length discrepancy after total hip arthroplasty. Journal of the American Academy of Orthopaedic Surgeons, 2006. 14(1): p. 38-45.

125. Gurney, B., Mermier, C., Robergs, R., Gibson, A., and Rivero, D., Effects of limb-length discrepancy on gait economy and lower-extremity muscle activity in older adults. The Journal of Bone \& Joint Surgery, 2001. 83(6): p. 907-915.

126. Edeen, J., Sharkey, P., and Alexander, A., Clinical significance of leg-length inequality after total hip arthroplasty. American journal of orthopedics (Belle Mead, NJ), 1995. 24(4): p. 347-351. 
127. Cristofolini, L., Viceconti, M., Toni, A., and Giunti, A., Influence of thigh muscles on the axial strains in a proximal femur during early stance in gait. Journal of biomechanics, 1995. 28(5): p. 617-624.

128. Keyak, J.H., Kaneko, T.S., Tehranzadeh, J., and Skinner, H.B., Predicting proximal femoral strength using structural engineering models. Clinical orthopaedics and related research, 2005. 437: p. 219-228.

129. Zdero, R., Rose, S., Schemitsch, E.H., and Papini, M., Cortical screw pullout strength and effective shear stress in synthetic third generation composite femurs. Journal of biomechanical engineering, 2007. 129(2): p. 289.

130. Zdero, R., Olsen, M., Bougherara, H., and Schemitsch, E., Cancellous bone screw purchase: a comparison of synthetic femurs, human femurs, and finite element analysis. Proceedings of the Institution of Mechanical Engineers, Part H: Journal of Engineering in Medicine, 2008. 222(8): p. 1175-1183.

131. Talbot, M., Zdero, R., and Schemitsch, E.H., Cyclic loading of periprosthetic fracture fixation constructs. Journal of Trauma-Injury, Infection, and Critical Care, 2008. 64(5): p. 1308-1312.

132. Courtney, A., Wachtel, E., Myers, E., and Hayes, W., Effects of loading rate on strength of the proximal femur. Calcified tissue international, 1994. 55(1): p. 53-58.

133. George, W. and Vashishth, D., Damage mechanisms and failure modes of cortical bone under components of physiological loading. Journal of orthopaedic research, 2005. 23(5): p. 1047-1053.

134. Yeh, O.C. and Keaveny, T.M., Relative roles of microdamage and microfracture in the mechanical behavior of trabecular bone. Journal of Orthopaedic Research, 2001. 19(6): p. 1001-1007.

135. Otani, T., Whiteside, L.A., and White, S.E., The effect of axial and torsional loading on strain distribution in the proximal femur as related to cementless total hip arthroplasty. Clinical orthopaedics and related research, 1993. 292: p. 376-383.

136. Rohlmann, A., Mössner, U., Bergmann, G., and Kölbel, R., Finite-element-analysis and experimental investigation of stresses in a femur. Journal of Biomedical Engineering, 1982. 4(3): p. 241-246.

137. Amstutz, H.C., Campbell, P.A., and Le Duff, M.J., Fracture of the neck of the femur after surface arthroplasty of the hip. The Journal of Bone \& Joint Surgery, 2004. 86(9): p. 1874-1877.

138. Cristofolini, L., Conti, G., Juszczyk, M., Cremonini, S., Sint Jan, S.V., and Viceconti, M., Structural behaviour and strain distribution of the long bones of the human lower limbs. Journal of Biomechanics, 2010. 43(5): p. 826-835. 
139. Abdul-Kadir, M.R., Hansen, U., Klabunde, R., Lucas, D., and Amis, A., Finite element modelling of primary hip stem stability: the effect of interference fit. Journal of biomechanics, 2008. 41(3): p. 587-594.

140. Bougherara, H., Bureau, M., Campbell, M., Vadean, A., and Yahia, L.H., Design of a biomimetic polymer-composite hip prosthesis. Journal of Biomedical Materials Research Part A, 2007. 82(1): p. 27-40.

141. Yamako, G., Chosa, E., Zhao, X., Totoribe, K., Watanabe, S., Sakamoto, T., and Nakane, N., Load-transfer analysis after insertion of cementless anatomical femoral stem using pre-and post-operative CT images based patient-specific finite element analysis. Medical engineering \& physics, 2014. 36(6): p. 694-700. 


\section{Appendix A: DOE Outcome Responses}

Neutral Phase Stress Outcome Responses:

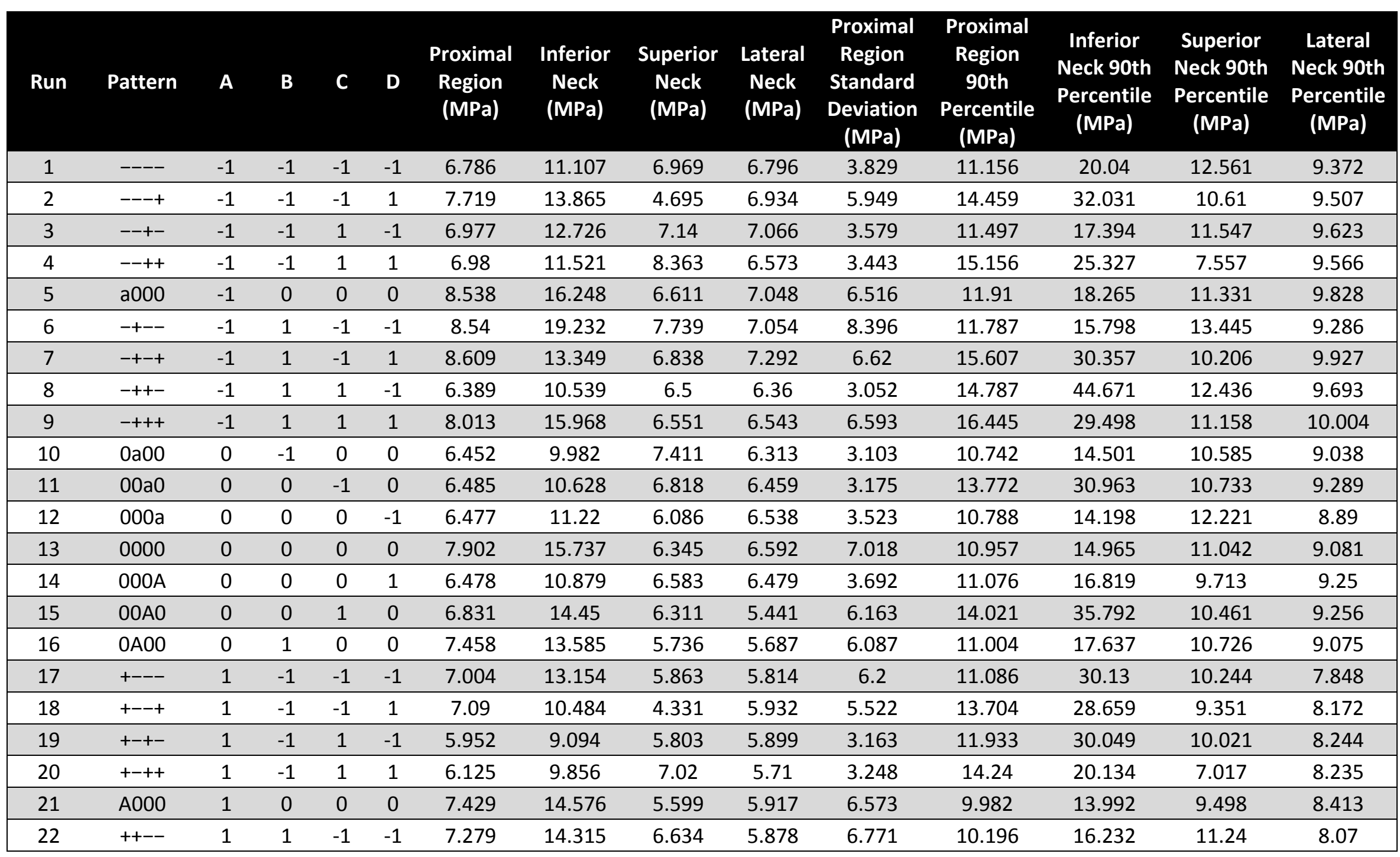




\begin{tabular}{|ccccccccccccccc|}
\hline 23 & ++-+ & 1 & 1 & -1 & 1 & 7.507 & 12.305 & 5.512 & 6.058 & 5.938 & 13.438 & 28.116 & 9.077 & 8.65 \\
\hline 24 & +++- & 1 & 1 & 1 & -1 & 6.786 & 11.107 & 6.969 & 6.796 & 3.829 & 12.988 & 34.61 & 10.771 & 8.213 \\
\hline 25 & ++++ & 1 & 1 & 1 & 1 & 7.719 & 13.865 & 4.695 & 6.934 & 5.949 & 14.686 & 25.849 & 9.061 & 8.32 \\
\hline $\begin{array}{l}\text { Intact } \\
\text { Values }\end{array}$ & & & & & & 5.128 & 9.190 & 6.113 & 4.057 & 2.995 & 9.191 & 13.612 & 9.663 & 5.877 \\
\hline
\end{tabular}


Neutral Phase \% of Intact Outcome Responses:

\begin{tabular}{|c|c|c|c|c|c|c|c|c|c|c|c|c|c|c|}
\hline Run & Pattern & A & B & C & D & $\begin{array}{c}\text { Proximal } \\
\text { Region } \\
(\%)\end{array}$ & $\begin{array}{c}\text { Inferior } \\
\text { Neck } \\
(\%)\end{array}$ & $\begin{array}{c}\text { Superior } \\
\text { Neck } \\
(\%)\end{array}$ & $\begin{array}{c}\text { Lateral } \\
\text { Neck } \\
(\%)\end{array}$ & $\begin{array}{c}\text { Proximal } \\
\text { Region } \\
\text { Standard } \\
\text { Deviation } \\
\text { (\%) }\end{array}$ & $\begin{array}{l}\text { Proximal } \\
\text { Region } \\
\text { 90th } \\
\text { Percentile } \\
\text { (\%) }\end{array}$ & $\begin{array}{c}\text { Inferior } \\
\text { Neck 90th } \\
\text { Percentile } \\
\text { (\%) }\end{array}$ & $\begin{array}{c}\text { Superior } \\
\text { Neck 90th } \\
\text { Percentile } \\
\text { (\%) }\end{array}$ & $\begin{array}{c}\text { Lateral } \\
\text { Neck 90th } \\
\text { Percentile } \\
\text { (\%) }\end{array}$ \\
\hline 1 & ---- & -1 & -1 & -1 & -1 & 132.02 & 131.75 & 124.14 & 161.57 & 123.49 & 121.38 & 147.22 & 129.98 & 159.48 \\
\hline 2 & ---+ & -1 & -1 & -1 & 1 & 159.96 & 165.04 & 107.28 & 164.22 & 223.17 & 157.33 & 235.31 & 109.80 & 161.78 \\
\hline 3 & --+- & -1 & -1 & 1 & -1 & 132.33 & 120.86 & 113.99 & 167.51 & 127.84 & 125.10 & 127.78 & 119.50 & 163.74 \\
\hline 4 & --++ & -1 & -1 & 1 & 1 & 150.53 & 150.87 & 76.80 & 170.91 & 198.62 & 164.91 & 186.06 & 78.20 & 162.79 \\
\hline 5 & $\mathrm{a} 000$ & -1 & 0 & 0 & 0 & 136.05 & 138.47 & 116.79 & 174.17 & 119.48 & 129.59 & 134.19 & 117.26 & 167.23 \\
\hline 6 & -+-- & -1 & 1 & -1 & -1 & 136.12 & 125.36 & 136.80 & 162.01 & 114.95 & 128.25 & 116.06 & 139.13 & 158.02 \\
\hline 7 & -+-+ & -1 & 1 & -1 & 1 & 166.49 & 176.80 & 108.14 & 173.73 & 217.57 & 169.82 & 223.02 & 105.61 & 168.93 \\
\hline 8 & -++- & -1 & 1 & 1 & -1 & 166.55 & 209.27 & 126.60 & 173.87 & 280.33 & 160.89 & 328.17 & 128.69 & 164.94 \\
\hline 9 & -+++ & -1 & 1 & 1 & 1 & 167.89 & 145.25 & 111.85 & 179.74 & 221.04 & 178.93 & 216.71 & 115.47 & 170.23 \\
\hline 10 & $0 \mathrm{a} 00$ & 0 & -1 & 0 & 0 & 124.59 & 114.68 & 106.33 & 156.76 & 101.89 & 116.88 & 106.53 & 109.53 & 153.79 \\
\hline 11 & $00 \mathrm{a} 0$ & 0 & 0 & -1 & 0 & 156.26 & 173.76 & 107.16 & 161.28 & 220.13 & 149.85 & 227.47 & 111.06 & 158.07 \\
\hline 12 & 000a & 0 & 0 & 0 & -1 & 125.83 & 108.62 & 121.23 & 155.62 & 103.60 & 117.38 & 104.31 & 126.47 & 151.27 \\
\hline 13 & 0000 & 0 & 0 & 0 & 0 & 126.47 & 115.64 & 111.53 & 159.20 & 106.02 & 119.22 & 109.94 & 114.27 & 154.53 \\
\hline 14 & $000 \mathrm{~A}$ & 0 & 0 & 0 & 1 & 126.32 & 122.09 & 99.55 & 161.14 & 117.64 & 120.52 & 123.56 & 100.51 & 157.40 \\
\hline 15 & OOAO & 0 & 0 & 1 & 0 & 154.10 & 171.24 & 103.78 & 162.47 & 234.32 & 152.55 & 262.94 & 108.25 & 157.51 \\
\hline 16 & OAO0 & 0 & 1 & 0 & 0 & 126.34 & 118.38 & 107.68 & 159.70 & 123.28 & 119.73 & 129.57 & 111.00 & 154.42 \\
\hline 17 & +---- & 1 & -1 & -1 & -1 & 133.21 & 157.24 & 103.23 & 134.12 & 205.79 & 120.63 & 221.35 & 106.01 & 133.54 \\
\hline 18 & +--+ & 1 & -1 & -1 & 1 & 145.44 & 147.83 & 93.83 & 140.19 & 203.23 & 149.10 & 210.54 & 96.76 & 139.06 \\
\hline 19 & +-+- & 1 & -1 & 1 & -1 & 136.58 & 143.14 & 95.90 & 143.32 & 207.01 & 129.83 & 220.76 & 103.70 & 140.29 \\
\hline 20 & +-++ & 1 & -1 & 1 & 1 & 138.26 & 114.08 & 70.84 & 146.22 & 184.36 & 154.94 & 147.91 & 72.61 & 140.13 \\
\hline 21 & $\mathrm{~A} 000$ & 1 & 0 & 0 & 0 & 116.07 & 98.95 & 94.93 & 145.40 & 105.61 & 108.61 & 102.79 & 98.29 & 143.16 \\
\hline 22 & ++-- & 1 & 1 & -1 & -1 & 119.44 & 107.24 & 114.83 & 140.74 & 108.43 & 110.94 & 119.25 & 116.31 & 137.32 \\
\hline
\end{tabular}




\begin{tabular}{|cccccccccccccc|c|}
23 & ++-+ & 1 & 1 & -1 & 1 & 144.88 & 158.61 & 91.58 & 145.85 & 219.45 & 146.21 & 206.55 & 93.93 & 147.19 \\
\hline 24 & +++- & 1 & 1 & 1 & -1 & 141.96 & 155.76 & 108.52 & 144.88 & 226.06 & 141.31 & 254.26 & 111.46 & 139.76 \\
\hline 25 & ++++ & 1 & 1 & 1 & 1 & 146.41 & 133.90 & 90.17 & 149.32 & 198.27 & 159.79 & 189.90 & 93.76 & 141.58 \\
\hline $\begin{array}{l}\text { Intact } \\
\text { Values }\end{array}$ & & & & & & 100 & 100 & 100 & 100 & 100 & 100 & 100 & 100 & 100 \\
\hline
\end{tabular}


Extension Phase Stress Outcome Responses:

\begin{tabular}{|c|c|c|c|c|c|c|c|c|c|c|c|c|c|c|}
\hline Run & Pattern & A & B & C & D & $\begin{array}{c}\text { Proximal } \\
\text { Region } \\
\text { (MPa) }\end{array}$ & $\begin{array}{c}\text { Inferior } \\
\text { Neck } \\
\text { (MPa) }\end{array}$ & $\begin{array}{c}\text { Superior } \\
\text { Neck } \\
\text { (MPa) }\end{array}$ & $\begin{array}{c}\text { Lateral } \\
\text { Neck } \\
\text { (MPa) }\end{array}$ & $\begin{array}{l}\text { Proximal } \\
\text { Region } \\
\text { Standard } \\
\text { Deviation } \\
\text { (MPa) }\end{array}$ & $\begin{array}{l}\text { Proximal } \\
\text { Region } \\
\text { 90th } \\
\text { Percentile } \\
\text { (MPa) }\end{array}$ & $\begin{array}{l}\text { Inferior } \\
\text { Neck 90th } \\
\text { Percentile } \\
\text { (MPa) }\end{array}$ & $\begin{array}{c}\text { Superior } \\
\text { Neck 90th } \\
\text { Percentile } \\
\text { (MPa) }\end{array}$ & $\begin{array}{c}\text { Lateral } \\
\text { Neck 90th } \\
\text { Percentile } \\
\text { (MPa) }\end{array}$ \\
\hline 1 & ---- & -1 & -1 & -1 & -1 & 7.451 & 13.331 & 7.932 & 6.624 & 4.174 & 12.816 & 21.809 & 13.108 & 9.523 \\
\hline 2 & ---+ & -1 & -1 & -1 & 1 & 9.118 & 16.416 & 6.834 & 6.841 & 8.220 & 16.923 & 34.723 & 11.407 & 9.767 \\
\hline 3 & --+- & -1 & -1 & 1 & -1 & 7.626 & 11.969 & 7.536 & 6.942 & 4.446 & 13.145 & 18.141 & 12.013 & 9.784 \\
\hline 5 & $\mathrm{a} 000$ & -1 & 0 & 0 & 0 & 7.766 & 13.868 & 7.680 & 7.137 & 4.274 & 13.752 & 20.544 & 12.177 & 10.086 \\
\hline 6 & -+-- & -1 & 1 & -1 & -1 & 7.797 & 12.852 & 8.906 & 6.634 & 4.173 & 13.619 & 18.443 & 14.824 & 9.509 \\
\hline 7 & -+-+ & -1 & 1 & -1 & 1 & 9.636 & 18.946 & 6.888 & 7.114 & 7.981 & 18.547 & 34.953 & 11.152 & 10.128 \\
\hline 8 & -++- & -1 & 1 & 1 & -1 & 9.821 & 20.631 & 8.643 & 7.180 & 9.633 & 17.335 & 44.750 & 13.445 & 10.291 \\
\hline 9 & -+++ & -1 & 1 & 1 & 1 & 9.942 & 15.878 & 7.287 & 7.433 & 8.442 & 18.628 & 31.564 & 11.976 & 10.435 \\
\hline 13 & 0000 & 0 & 0 & 0 & 0 & 7.375 & 11.917 & 7.383 & 6.676 & 3.944 & 12.897 & 17.347 & 11.828 & 9.611 \\
\hline 14 & 000A & 0 & 0 & 0 & 1 & 7.299 & 12.177 & 6.442 & 6.724 & 4.290 & 13.019 & 18.645 & 10.268 & 9.653 \\
\hline 15 & OOAO & 0 & 0 & 1 & 0 & 8.993 & 16.963 & 7.050 & 6.823 & 8.156 & 16.037 & 36.438 & 11.027 & 9.642 \\
\hline 16 & OAO0 & 0 & 1 & 0 & 0 & 7.483 & 12.143 & 7.216 & 6.705 & 4.505 & 13.220 & 19.108 & 11.750 & 9.685 \\
\hline 17 & +--- & 1 & -1 & -1 & -1 & 7.965 & 15.379 & 6.757 & 5.715 & 7.327 & 13.657 & 27.581 & 10.653 & 8.392 \\
\hline 18 & +--+ & 1 & -1 & -1 & 1 & 8.391 & 15.272 & 5.797 & 5.915 & 7.491 & 16.178 & 32.166 & 10.067 & 8.450 \\
\hline 19 & +-+- & 1 & -1 & 1 & -1 & 8.423 & 13.928 & 6.522 & 6.165 & 8.412 & 14.639 & 29.165 & 10.578 & 8.801 \\
\hline 20 & +-++ & 1 & -1 & 1 & 1 & 8.165 & 11.830 & 4.994 & 6.325 & 6.613 & 16.302 & 22.015 & 7.743 & 8.793 \\
\hline 21 & $\mathrm{~A} 000$ & 1 & 0 & 0 & 0 & 6.897 & 10.085 & 6.286 & 6.210 & 4.006 & 12.056 & 15.816 & 10.228 & 8.943 \\
\hline 22 & ++-- & 1 & 1 & -1 & -1 & 7.324 & 12.045 & 7.903 & 6.013 & 4.111 & 12.782 & 19.289 & 12.708 & 8.906 \\
\hline
\end{tabular}




\begin{tabular}{|ccccccccccccccc|}
23 & ++-+ & 1 & 1 & -1 & 1 & 7.772 & 13.820 & 5.997 & 6.087 & 5.660 & 14.172 & 22.496 & 9.855 & 8.789 \\
\hline 24 & +++- & 1 & 1 & 1 & -1 & 8.891 & 15.584 & 7.608 & 6.266 & 8.780 & 15.911 & 34.618 & 11.911 & 9.164 \\
\hline 25 & ++++ & 1 & 1 & 1 & 1 & 8.787 & 14.266 & 6.252 & 6.425 & 7.344 & 16.900 & 28.270 & 10.123 & 9.124 \\
\hline $\begin{array}{l}\text { Intact } \\
\text { Values }\end{array}$ & & & & & & 6.096 & 10.564 & 7.168 & 4.640 & 3.391 & 10.740 & 16.470 & 11.361 & 6.703 \\
\hline
\end{tabular}


Extension Phase \% of Intact Outcome Responses:

\begin{tabular}{|c|c|c|c|c|c|c|c|c|c|c|c|c|c|c|}
\hline Run & Pattern & $\mathbf{A}$ & B & C & D & $\begin{array}{c}\text { Proximal } \\
\text { Region } \\
(\%)\end{array}$ & $\begin{array}{c}\text { Inferior } \\
\text { Neck } \\
(\%)\end{array}$ & $\begin{array}{c}\text { Superior } \\
\text { Neck } \\
(\%)\end{array}$ & $\begin{array}{c}\text { Lateral } \\
\text { Neck } \\
(\%)\end{array}$ & $\begin{array}{l}\text { Proximal } \\
\text { Region } \\
\text { Standard } \\
\text { Deviation } \\
\text { (\%) }\end{array}$ & $\begin{array}{l}\text { Proximal } \\
\text { Region } \\
\text { 90th } \\
\text { Percentile } \\
\text { (\%) }\end{array}$ & $\begin{array}{c}\text { Inferior } \\
\text { Neck 90th } \\
\text { Percentile } \\
\text { (\%) }\end{array}$ & $\begin{array}{c}\text { Superior } \\
\text { Neck 90th } \\
\text { Percentile } \\
\text { (\%) }\end{array}$ & $\begin{array}{c}\text { Lateral } \\
\text { Neck 90th } \\
\text { Percentile } \\
\text { (\%) }\end{array}$ \\
\hline 1 & ---- & -1 & -1 & -1 & -1 & 122.23 & 126.20 & 110.66 & 142.75 & 123.10 & 119.32 & 132.42 & 115.38 & 142.07 \\
\hline 2 & ---+ & -1 & -1 & -1 & 1 & 149.58 & 155.40 & 95.34 & 147.43 & 242.43 & 157.57 & 210.83 & 100.40 & 145.70 \\
\hline 3 & --+- & -1 & -1 & 1 & -1 & 125.10 & 113.30 & 105.13 & 149.60 & 131.14 & 122.39 & 110.15 & 105.74 & 145.96 \\
\hline 4 & --++ & -1 & -1 & 1 & 1 & 158.86 & 167.38 & 86.99 & 152.91 & 241.77 & 172.51 & 185.99 & 87.82 & 147.34 \\
\hline 5 & $\mathrm{a} 000$ & -1 & 0 & 0 & 0 & 127.39 & 131.28 & 107.14 & 153.81 & 126.07 & 128.04 & 124.74 & 107.18 & 150.46 \\
\hline 6 & -+-- & -1 & 1 & -1 & -1 & 127.91 & 121.66 & 124.24 & 142.96 & 123.07 & 126.80 & 111.98 & 130.48 & 141.86 \\
\hline 7 & -+-+ & -1 & 1 & -1 & 1 & 158.08 & 179.35 & 96.08 & 153.30 & 235.38 & 172.69 & 212.23 & 98.16 & 151.09 \\
\hline 8 & -++- & -1 & 1 & 1 & -1 & 161.10 & 195.30 & 120.57 & 154.74 & 284.10 & 161.40 & 271.71 & 118.34 & 153.53 \\
\hline 9 & -+++ & -1 & 1 & 1 & 1 & 163.10 & 150.30 & 101.65 & 160.18 & 248.97 & 173.44 & 191.65 & 105.41 & 155.68 \\
\hline 10 & $0 \mathrm{aO0}$ & 0 & -1 & 0 & 0 & 117.54 & 109.45 & 96.28 & 141.46 & 109.69 & 115.57 & 99.96 & 97.18 & 139.60 \\
\hline 11 & $00 \mathrm{aO}$ & 0 & 0 & -1 & 0 & 148.24 & 166.68 & 95.16 & 143.77 & 236.87 & 154.62 & 204.19 & 103.08 & 143.11 \\
\hline 12 & $000 a$ & 0 & 0 & 0 & -1 & 121.65 & 107.72 & 113.25 & 139.63 & 113.09 & 118.55 & 99.45 & 115.21 & 140.99 \\
\hline 13 & 0000 & 0 & 0 & 0 & 0 & 120.99 & 112.81 & 102.99 & 143.88 & 116.34 & 120.08 & 105.33 & 104.10 & 143.37 \\
\hline 14 & $000 \mathrm{~A}$ & 0 & 0 & 0 & 1 & 119.74 & 115.27 & 89.86 & 144.90 & 126.52 & 121.21 & 113.21 & 90.37 & 144.00 \\
\hline 15 & OOAO & 0 & 0 & 1 & 0 & 147.53 & 160.58 & 98.35 & 147.03 & 240.56 & 149.32 & 221.25 & 97.05 & 143.84 \\
\hline 16 & OAO0 & 0 & 1 & 0 & 0 & 122.76 & 114.95 & 100.66 & 144.49 & 132.88 & 123.09 & 116.02 & 103.42 & 144.49 \\
\hline 17 & +-- & 1 & -1 & -1 & -1 & 130.67 & 145.59 & 94.27 & 123.15 & 216.11 & 127.16 & 167.47 & 93.77 & 125.20 \\
\hline 18 & +--+ & 1 & -1 & -1 & 1 & 137.64 & 144.57 & 80.87 & 127.47 & 220.93 & 150.62 & 195.30 & 88.60 & 126.06 \\
\hline 19 & +-+- & 1 & -1 & 1 & -1 & 138.18 & 131.85 & 90.99 & 132.85 & 248.10 & 136.30 & 177.08 & 93.10 & 131.29 \\
\hline 20 & +-++ & 1 & -1 & 1 & 1 & 133.93 & 111.99 & 69.67 & 136.30 & 195.03 & 151.79 & 133.67 & 68.15 & 131.17 \\
\hline 21 & $\mathrm{~A} 000$ & 1 & 0 & 0 & 0 & 113.14 & 95.47 & 87.69 & 133.82 & 118.16 & 112.25 & 96.03 & 90.03 & 133.42 \\
\hline 22 & ++-- & 1 & 1 & -1 & -1 & 120.15 & 114.02 & 110.25 & 129.57 & 121.26 & 119.01 & 117.12 & 111.85 & 132.86 \\
\hline
\end{tabular}




\begin{tabular}{|ccccccc|ccccccc|c|}
\hline 23 & ++-+ & 1 & 1 & -1 & 1 & 127.49 & 130.82 & 83.66 & 131.17 & 166.92 & 131.95 & 136.59 & 86.74 & 131.12 \\
\hline 24 & +++- & 1 & 1 & 1 & -1 & 145.85 & 147.52 & 106.13 & 135.03 & 258.94 & 148.15 & 210.19 & 104.84 & 136.70 \\
\hline 25 & ++++ & 1 & 1 & 1 & 1 & 144.14 & 135.05 & 87.21 & 138.46 & 216.59 & 157.35 & 171.65 & 89.10 & 136.11 \\
\hline $\begin{array}{l}\text { Intact } \\
\text { Values }\end{array}$ & & & & & & 100 & 100 & 100 & 100 & 100 & 100 & 100 & 100 & 100 \\
\hline
\end{tabular}


Flexion Phase Stress Outcome Responses:

\begin{tabular}{|c|c|c|c|c|c|c|c|c|c|c|c|c|c|c|}
\hline Run & Pattern & A & B & C & D & $\begin{array}{c}\text { Proximal } \\
\text { Region } \\
\text { (MPa) }\end{array}$ & $\begin{array}{l}\text { Inferior } \\
\text { Neck } \\
\text { (MPa) }\end{array}$ & $\begin{array}{c}\text { Superior } \\
\text { Neck } \\
\text { (MPa) }\end{array}$ & $\begin{array}{c}\text { Lateral } \\
\text { Neck } \\
\text { (MPa) }\end{array}$ & $\begin{array}{l}\text { Proximal } \\
\text { Region } \\
\text { Standard } \\
\text { Deviation } \\
\text { (MPa) }\end{array}$ & $\begin{array}{l}\text { Proximal } \\
\text { Region } \\
\text { 90th } \\
\text { Percentile } \\
\text { (MPa) }\end{array}$ & $\begin{array}{c}\text { Inferior } \\
\text { Neck 90th } \\
\text { Percentile } \\
\text { (MPa) }\end{array}$ & $\begin{array}{c}\text { Superior } \\
\text { Neck 90th } \\
\text { Percentile } \\
\text { (MPa) }\end{array}$ & $\begin{array}{l}\text { Lateral } \\
\text { Neck 90th } \\
\text { Percentile } \\
\text { (MPa) }\end{array}$ \\
\hline 1 & ---- & -1 & -1 & -1 & -1 & 6.728 & 12.129 & 7.337 & 6.157 & 4.102 & 11.628 & 20.634 & 11.966 & 9.304 \\
\hline 2 & ---+ & -1 & -1 & -1 & 1 & 8.600 & 17.290 & 6.363 & 6.329 & 7.890 & 15.083 & 32.304 & 9.953 & 9.683 \\
\hline 5 & $\mathrm{a} 000$ & -1 & 0 & 0 & 0 & 6.642 & 12.738 & 6.544 & 6.591 & 3.773 & 11.689 & 18.303 & 10.632 & 9.588 \\
\hline 6 & -+-- & -1 & 1 & -1 & -1 & 6.668 & 11.215 & 7.785 & 6.117 & 3.526 & 11.263 & 15.865 & 12.507 & 9.063 \\
\hline 7 & -+-+ & -1 & 1 & -1 & 1 & 8.666 & 18.508 & 6.093 & 6.542 & 7.780 & 16.002 & 33.852 & 9.467 & 9.543 \\
\hline 8 & -++- & -1 & 1 & 1 & -1 & 8.282 & 20.038 & 6.695 & 6.511 & 9.144 & 14.133 & 44.457 & 12.034 & 9.317 \\
\hline 9 & -+++ & -1 & 1 & 1 & 1 & 8.510 & 13.445 & 6.108 & 6.704 & 8.276 & 16.678 & 30.371 & 10.094 & 9.464 \\
\hline 13 & 0000 & 0 & 0 & 0 & 0 & 6.211 & 10.681 & 6.350 & 5.910 & 3.356 & 10.793 & 15.452 & 10.422 & 8.812 \\
\hline 14 & $000 \mathrm{~A}$ & 0 & 0 & 0 & 1 & 6.190 & 11.610 & 5.689 & 5.994 & 3.700 & 11.012 & 17.386 & 9.090 & 8.965 \\
\hline 15 & OOAO & 0 & 0 & 1 & 0 & 7.807 & 17.350 & 5.617 & 5.975 & 8.083 & 13.848 & 36.754 & 10.156 & 8.964 \\
\hline 16 & $0 A 00$ & 0 & 1 & 0 & 0 & 6.069 & 11.048 & 6.064 & 5.927 & 3.650 & 10.700 & 16.967 & 10.243 & 8.822 \\
\hline 17 & +--- & 1 & -1 & -1 & -1 & 7.297 & 16.043 & 6.239 & 5.084 & 7.732 & 11.859 & 35.636 & 10.091 & 7.948 \\
\hline 18 & +--+ & 1 & -1 & -1 & 1 & 7.589 & 14.306 & 5.619 & 5.222 & 7.059 & 13.453 & 28.281 & 8.945 & 8.280 \\
\hline 19 & +-+- & 1 & -1 & 1 & -1 & 7.175 & 14.669 & 5.691 & 5.355 & 7.230 & 12.091 & 30.063 & 10.320 & 8.357 \\
\hline 20 & +-++ & 1 & -1 & 1 & 1 & 6.121 & 12.522 & 3.824 & 4.877 & 5.341 & 11.321 & 23.967 & 6.278 & 7.475 \\
\hline 21 & A000 & 1 & 0 & 0 & 0 & 5.752 & 9.602 & 5.532 & 5.334 & 3.276 & 10.029 & 14.407 & 8.963 & 8.300 \\
\hline 22 & ++-- & 1 & 1 & -1 & -1 & 5.722 & 8.908 & 6.269 & 5.160 & 3.264 & 9.701 & 14.390 & 10.494 & 7.719 \\
\hline
\end{tabular}




\begin{tabular}{|ccccccccccccccc|}
23 & ++-+ & 1 & 1 & -1 & 1 & 5.845 & 11.621 & 4.743 & 5.236 & 4.753 & 10.068 & 22.153 & 7.482 & 7.852 \\
\hline 24 & +++- & 1 & 1 & 1 & -1 & 7.153 & 15.569 & 5.783 & 5.278 & 7.559 & 12.468 & 34.029 & 11.175 & 7.893 \\
\hline 25 & ++++ & 1 & 1 & 1 & 1 & 7.461 & 13.525 & 4.777 & 5.411 & 7.221 & 15.159 & 26.963 & 7.924 & 8.127 \\
\hline $\begin{array}{l}\text { Intact } \\
\text { Values }\end{array}$ & & & & & & 5.452 & 9.997 & 6.345 & 4.029 & 3.347 & 9.813 & 14.388 & 10.163 & 6.212 \\
\hline
\end{tabular}


Flexion Phase \% of Intact Outcome Responses:

\begin{tabular}{|c|c|c|c|c|c|c|c|c|c|c|c|c|c|c|}
\hline Run & Pattern & A & B & C & D & $\begin{array}{c}\text { Proximal } \\
\text { Region } \\
\text { (\%) }\end{array}$ & $\begin{array}{c}\text { Inferior } \\
\text { Neck } \\
(\%)\end{array}$ & $\begin{array}{c}\text { Superior } \\
\text { Neck } \\
\text { (\%) }\end{array}$ & $\begin{array}{c}\text { Lateral } \\
\text { Neck } \\
\text { (\%) }\end{array}$ & $\begin{array}{l}\text { Proximal } \\
\text { Region } \\
\text { Standard } \\
\text { Deviation } \\
\text { (\%) }\end{array}$ & $\begin{array}{l}\text { Proximal } \\
\text { Region } \\
\text { 90th } \\
\text { Percentile } \\
\text { (\%) }\end{array}$ & $\begin{array}{c}\text { Inferior } \\
\text { Neck 90th } \\
\text { Percentile } \\
\text { (\%) }\end{array}$ & $\begin{array}{c}\text { Superior } \\
\text { Neck 90th } \\
\text { Percentile } \\
\text { (\%) }\end{array}$ & $\begin{array}{c}\text { Lateral } \\
\text { Neck 90th } \\
\text { Percentile } \\
\text { (\%) }\end{array}$ \\
\hline 1 & ---- & -1 & -1 & -1 & -1 & 123.41 & 121.32 & 115.63 & 152.80 & 122.57 & 118.49 & 143.41 & 117.74 & 149.76 \\
\hline 2 & ---+ & -1 & -1 & -1 & 1 & 157.75 & 172.95 & 100.28 & 157.08 & 235.77 & 153.70 & 224.52 & 97.93 & 155.87 \\
\hline 5 & $\mathrm{a} 000$ & -1 & 0 & 0 & 0 & 121.83 & 127.42 & 103.14 & 163.58 & 112.74 & 119.11 & 127.21 & 104.61 & 154.33 \\
\hline 6 & -+-- & -1 & 1 & -1 & -1 & 122.30 & 112.19 & 122.69 & 151.82 & 105.36 & 114.77 & 110.27 & 123.06 & 145.90 \\
\hline 7 & -+-+ & -1 & 1 & -1 & 1 & 158.96 & 185.13 & 96.02 & 162.37 & 232.47 & 163.06 & 235.28 & 93.14 & 153.61 \\
\hline 8 & -++- & -1 & 1 & 1 & -1 & 151.91 & 200.44 & 105.52 & 161.59 & 273.23 & 144.02 & 308.99 & 118.40 & 149.98 \\
\hline 9 & -+++ & -1 & 1 & 1 & 1 & 156.09 & 134.49 & 96.26 & 166.40 & 247.30 & 169.95 & 211.09 & 99.31 & 152.34 \\
\hline 13 & 0000 & 0 & 0 & 0 & 0 & 113.93 & 106.85 & 100.07 & 146.69 & 100.29 & 109.98 & 107.40 & 102.54 & 141.86 \\
\hline 14 & O00A & 0 & 0 & 0 & 1 & 113.54 & 116.14 & 89.66 & 148.76 & 110.55 & 112.21 & 120.84 & 89.44 & 144.31 \\
\hline 15 & OOAO & 0 & 0 & 1 & 0 & 143.20 & 173.55 & 88.52 & 148.29 & 241.52 & 141.12 & 255.45 & 99.93 & 144.30 \\
\hline 16 & OA00 & 0 & 1 & 0 & 0 & 111.33 & 110.52 & 95.56 & 147.09 & 109.07 & 109.03 & 117.92 & 100.78 & 142.01 \\
\hline 17 & +--- & 1 & -1 & -1 & -1 & 133.86 & 160.48 & 98.33 & 126.17 & 231.03 & 120.85 & 247.68 & 99.29 & 127.94 \\
\hline 18 & +--+ & 1 & -1 & -1 & 1 & 139.20 & 143.10 & 88.55 & 129.60 & 210.93 & 137.09 & 196.57 & 88.01 & 133.28 \\
\hline 19 & +-+- & 1 & -1 & 1 & -1 & 131.61 & 146.74 & 89.69 & 132.91 & 216.04 & 123.20 & 208.95 & 101.54 & 134.53 \\
\hline 20 & +-++ & 1 & -1 & 1 & 1 & 112.27 & 125.26 & 60.27 & 121.05 & 159.59 & 115.36 & 166.58 & 61.77 & 120.33 \\
\hline 21 & $\mathrm{~A} 000$ & 1 & 0 & 0 & 0 & 105.52 & 96.04 & 87.19 & 132.39 & 97.90 & 102.20 & 100.13 & 88.19 & 133.61 \\
\hline 22 & ++-- & 1 & 1 & -1 & -1 & 104.97 & 89.11 & 98.79 & 128.06 & 97.52 & 98.85 & 100.01 & 103.25 & 124.26 \\
\hline
\end{tabular}




\begin{tabular}{|cccccccccccccc|c|}
\hline 23 & ++-+ & 1 & 1 & -1 & 1 & 107.21 & 116.25 & 74.76 & 129.96 & 142.02 & 102.59 & 153.97 & 73.62 & 126.39 \\
\hline 24 & +++- & 1 & 1 & 1 & -1 & 131.21 & 155.74 & 91.14 & 131.00 & 225.88 & 127.05 & 236.51 & 109.96 & 127.05 \\
\hline 25 & ++++ & 1 & 1 & 1 & 1 & 136.86 & 135.29 & 75.28 & 134.29 & 215.77 & 154.47 & 187.40 & 77.96 & 130.82 \\
\hline $\begin{array}{l}\text { Intact } \\
\text { Values }\end{array}$ & & & & & & 100 & 100 & 100 & 100 & 100 & 100 & 100 & 100 & 100 \\
\hline
\end{tabular}




\section{Appendix B: Weighted Averages for Each Gait Phase}

Neutral Phase:

\begin{tabular}{|c|c|c|c|c|c|c|c|c|c|c|c|c|}
\hline $\begin{array}{l}\text { Response } \\
\text { Selection }\end{array}$ & $\begin{array}{c}\text { A } \\
\text { Weight }\end{array}$ & $\begin{array}{c}\text { A } \\
\text { Factor } \\
\text { Setting } \\
\left({ }^{\circ}\right)\end{array}$ & $\begin{array}{c}\text { A } \\
\text { Weighted } \\
\text { Value }\end{array}$ & $\begin{array}{c}\text { B } \\
\text { Weight }\end{array}$ & $\begin{array}{c}\text { B } \\
\text { Factor } \\
\text { Setting } \\
\left(^{\circ}\right)\end{array}$ & $\begin{array}{c}\text { B } \\
\text { Weighted } \\
\text { Value }\end{array}$ & $\begin{array}{c}\text { C } \\
\text { Weight }\end{array}$ & $\begin{array}{c}\text { C } \\
\text { Factor } \\
\text { Setting } \\
(\mathbf{m m})\end{array}$ & $\begin{array}{c}\text { C } \\
\text { Weighted } \\
\text { Value }\end{array}$ & $\begin{array}{c}\text { D } \\
\text { Weight }\end{array}$ & $\begin{array}{c}\text { D } \\
\text { Factor } \\
\text { Setting } \\
(\mathrm{mm})\end{array}$ & $\begin{array}{l}\text { D Weighted } \\
\text { Value }\end{array}$ \\
\hline $\begin{array}{l}\text { Approach } 1 \\
\text { Method } 1\end{array}$ & 0.25 & 3.15 & 0.79 & 0.38 & 0.00 & 0.00 & 0.10 & 1.13 & 0.11 & 0.38 & 0.00 & 0.00 \\
\hline $\begin{array}{l}\text { Approach } 1 \\
\text { Method } 2\end{array}$ & 0.19 & 10.00 & 1.88 & 0.13 & 0.00 & 0.00 & 0.25 & 0.78 & 0.19 & 0.30 & 0.00 & 0.00 \\
\hline $\begin{array}{l}\text { Approach } 2 \\
\text { Method } 1\end{array}$ & 0.30 & 8.05 & 2.42 & 0.30 & 0.00 & 0.00 & 0.10 & 0.32 & 0.03 & 0.05 & 0.00 & 0.00 \\
\hline $\begin{array}{l}\text { Approach } 2 \\
\text { Method } 2\end{array}$ & 0.45 & 10.00 & 4.50 & 0.15 & 0.00 & 0.00 & 0.25 & -0.11 & -0.03 & 0.25 & 0.12 & 0.03 \\
\hline $\begin{array}{l}\text { Approach } 3 \\
\text { Method } 1\end{array}$ & 0.45 & 10.00 & 4.50 & 0.15 & 0.00 & 0.00 & 0.25 & -0.43 & -0.11 & 0.20 & 1.39 & 0.28 \\
\hline $\begin{array}{l}\text { Approach } 3 \\
\text { Method } 2\end{array}$ & 0.45 & 10.00 & 4.50 & 0.23 & 0.00 & 0.00 & 0.25 & -0.31 & -0.08 & 0.20 & 1.23 & 0.25 \\
\hline Total & 2.09 & --- & $8.90^{\circ}$ & 1.33 & --- & $0.00^{\circ}$ & 1.20 & --- & $0.11 \mathrm{~mm}$ & 1.38 & --- & $0.40 \mathrm{~mm}$ \\
\hline
\end{tabular}




\section{Extension Phase:}

\begin{tabular}{|c|c|c|c|c|c|c|c|c|c|c|c|c|}
\hline $\begin{array}{l}\text { Response } \\
\text { Selection }\end{array}$ & $\begin{array}{c}\text { A } \\
\text { Weight }\end{array}$ & $\begin{array}{c}\text { A Factor } \\
\text { Setting } \\
\left({ }^{\circ}\right)\end{array}$ & $\begin{array}{c}\text { A } \\
\text { Weighted } \\
\text { Value }\end{array}$ & $\begin{array}{c}\text { B } \\
\text { Weight }\end{array}$ & $\begin{array}{c}\text { B } \\
\text { Factor } \\
\text { Setting } \\
\left({ }^{\circ}\right)\end{array}$ & $\begin{array}{c}\text { B } \\
\text { Weighted } \\
\text { Value }\end{array}$ & $\begin{array}{c}\text { C } \\
\text { Weight }\end{array}$ & $\begin{array}{c}\text { C } \\
\text { Factor } \\
\text { Setting } \\
(\mathrm{mm})\end{array}$ & $\begin{array}{c}\text { C } \\
\text { Weighted } \\
\text { Value }\end{array}$ & $\begin{array}{c}\text { D } \\
\text { Weight }\end{array}$ & $\begin{array}{c}\text { D } \\
\text { Factor } \\
\text { Setting } \\
(\mathrm{mm})\end{array}$ & $\begin{array}{c}\text { D } \\
\text { Weighted } \\
\text { Value }\end{array}$ \\
\hline $\begin{array}{l}\text { Approach } 1 \\
\text { Method } 1\end{array}$ & 0.20 & -0.98 & -0.20 & 0.15 & 0.00 & 0.00 & 0.65 & -0.17 & -0.11 & 0.45 & 0.00 & 0.00 \\
\hline $\begin{array}{l}\text { Approach } 1 \\
\text { Method } 2\end{array}$ & 0.20 & -0.88 & -0.18 & 0.15 & 0.00 & 0.00 & 0.60 & -0.17 & -0.10 & 0.42 & 0.00 & 0.00 \\
\hline $\begin{array}{l}\text { Approach } 2 \\
\text { Method } 1\end{array}$ & 0.45 & 10.00 & 4.50 & 0.23 & 0.00 & 0.00 & 0.35 & -0.82 & -0.29 & 0.35 & 0.00 & 0.00 \\
\hline $\begin{array}{l}\text { Approach } 2 \\
\text { Method } 2\end{array}$ & 0.45 & 10.00 & 4.50 & 0.15 & 1.89 & 0.28 & 0.25 & -0.44 & -0.11 & 0.38 & 0.00 & 0.00 \\
\hline $\begin{array}{l}\text { Approach } 3 \\
\text { Method } 1\end{array}$ & 0.45 & 10.00 & 4.50 & 0.23 & 0.00 & 0.00 & 0.25 & -0.93 & -0.23 & 0.30 & 0.00 & 0.00 \\
\hline $\begin{array}{l}\text { Approach } 3 \\
\text { Method } 2\end{array}$ & 0.42 & 10.00 & 4.20 & 0.15 & 0.00 & 0.00 & 0.25 & -0.91 & -0.23 & 0.30 & 0.00 & 0.00 \\
\hline Total & 2.17 & --- & $7.99^{\circ}$ & 1.05 & --- & $0.27^{\circ}$ & 2.35 & --- & $-0.46 \mathrm{~mm}$ & 2.20 & --- & $0.00 \mathrm{~mm}$ \\
\hline
\end{tabular}


Flexion Phase:

\begin{tabular}{|c|c|c|c|c|c|c|c|c|c|c|c|c|}
\hline $\begin{array}{l}\text { Response } \\
\text { Selection }\end{array}$ & $\begin{array}{c}\text { A } \\
\text { Weight }\end{array}$ & $\begin{array}{c}\text { A } \\
\text { Factor } \\
\text { Setting } \\
\left(^{\circ}\right)\end{array}$ & $\begin{array}{c}\text { A } \\
\text { Weighted } \\
\text { Value }\end{array}$ & $\begin{array}{c}\text { B } \\
\text { Weight }\end{array}$ & $\begin{array}{c}\text { B } \\
\text { Factor } \\
\text { Setting } \\
\left(^{\circ}\right)\end{array}$ & $\begin{array}{c}\text { B } \\
\text { Weighted } \\
\text { Value }\end{array}$ & $\begin{array}{c}\text { C } \\
\text { Weight }\end{array}$ & $\begin{array}{c}\text { C } \\
\text { Factor } \\
\text { Setting } \\
(\mathrm{mm})\end{array}$ & $\begin{array}{c}\text { C } \\
\text { Weighted } \\
\text { Value }\end{array}$ & $\begin{array}{c}\text { D } \\
\text { Weight }\end{array}$ & $\begin{array}{c}\text { D } \\
\text { Factor } \\
\text { Setting } \\
(\mathbf{m m})\end{array}$ & $\begin{array}{c}\text { D } \\
\text { Weighted } \\
\text { Value }\end{array}$ \\
\hline $\begin{array}{l}\text { Approach } 1 \\
\text { Method } 1\end{array}$ & 0.42 & 10.00 & 4.20 & 0.00 & 10.00 & 0.00 & 0.70 & 0.00 & 0.00 & 0.00 & 1.88 & 0.00 \\
\hline $\begin{array}{l}\text { Approach } 1 \\
\text { Method } 2\end{array}$ & 0.41 & 10.00 & 4.05 & 0.00 & 10.00 & 0.00 & 0.73 & 0.01 & 0.01 & 0.00 & 1.85 & 0.00 \\
\hline $\begin{array}{l}\text { Approach } 2 \\
\text { Method } 1\end{array}$ & 0.75 & 10.00 & 7.50 & 0.15 & 10.00 & 1.50 & 0.42 & -1.55 & -0.65 & 0.30 & 0.00 & 0.00 \\
\hline $\begin{array}{l}\text { Approach } 2 \\
\text { Method } 2\end{array}$ & 0.68 & 10.00 & 6.75 & 0.15 & 10.00 & 1.50 & 0.35 & -1.55 & -0.54 & 0.45 & 0.00 & 0.00 \\
\hline $\begin{array}{l}\text { Approach } 3 \\
\text { Method } 1\end{array}$ & 0.68 & 10.00 & 6.75 & 0.23 & 10.00 & 2.25 & 0.35 & -1.38 & -0.48 & 0.30 & 0.90 & 0.27 \\
\hline $\begin{array}{l}\text { Approach } 3 \\
\text { Method } 2\end{array}$ & 0.60 & 10.00 & 6.00 & 0.23 & 10.00 & 2.25 & 0.35 & -1.38 & -0.48 & 0.33 & 0.89 & 0.29 \\
\hline Total & 3.53 & --- & $10.00^{\circ}$ & 0.75 & --- & $10.00^{\circ}$ & 2.90 & --- & $-0.74 \mathrm{~mm}$ & 1.38 & -- & $0.41 \mathrm{~mm}$ \\
\hline
\end{tabular}




\section{Appendix C: Complete Neutral Phase DOE Results Approach 1 Outcomes - Proximal Region}

\section{Method 1 - Mean Stress Model:}

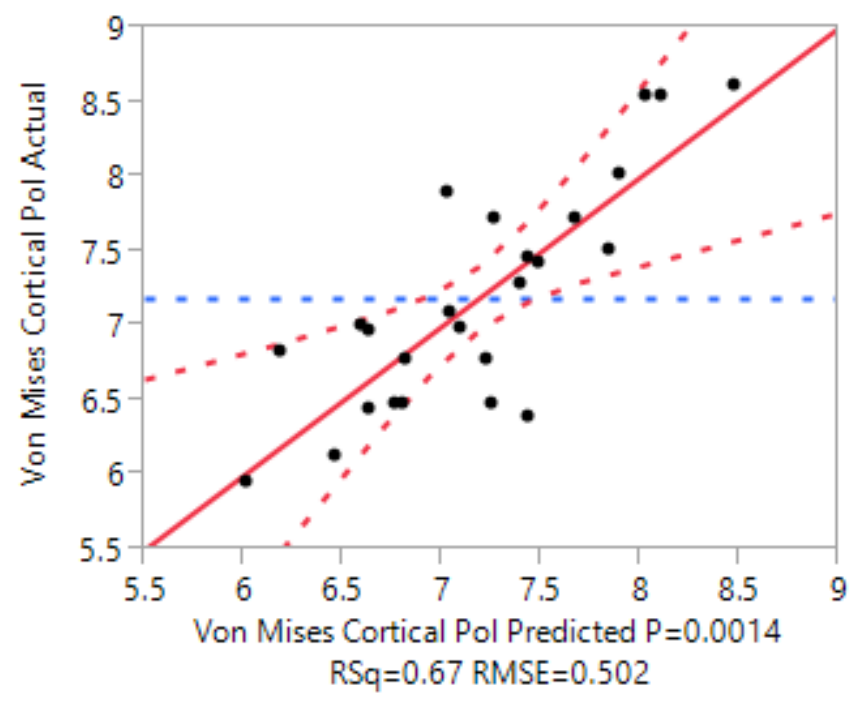

\section{Summary of Fit}

\begin{tabular}{l|r|}
\hline RSquare & 0.666204 \\
RSquare Adj & 0.554939 \\
Root Mean Square Error & 0.501962 \\
Mean of Response & 7.181 \\
Observations (or Sum Wgts) & 25
\end{tabular}

\begin{tabular}{|c|c|c|c|c|}
\hline \multicolumn{5}{|c|}{ Analysis of Variance } \\
\hline Source & DF & $\begin{array}{r}\text { Sum of } \\
\text { Squares }\end{array}$ & Mean Square & F Ratio \\
\hline Model & 6 & 9.051900 & 1.50865 & 5.9875 \\
\hline Error & 18 & 4.535380 & 0.25197 & Prob $>F$ \\
\hline C. Total & 24 & 13.587280 & & $0.0014^{*}$ \\
\hline
\end{tabular}

\section{Parameter Estimates}

\begin{tabular}{lrrrrrrr}
\hline Term & Estimate & Std Error & t Ratio & Prob $>|\mathbf{t}|$ & Lower 95\% & Upper 95\% \\
Intercept & 7.028 & 0.205937 & 34.13 & $<.0001^{*}$ & 6.5953424 & 7.4606576 \\
A & -0.314444 & 0.118314 & -2.66 & $0.0160^{*}$ & -0.563012 & -0.065877 \\
B & 0.4008333 & 0.118314 & 3.39 & $0.0033^{*}$ & 0.1522659 & 0.6494008 \\
C & -0.2915 & 0.118314 & -2.46 & $0.0240^{*}$ & -0.540067 & -0.042933 \\
D & 0.225 & 0.118314 & 1.90 & 0.0733 & -0.023567 & 0.4735675 \\
A $^{\star} \mathrm{A}$ & 0.769 & 0.280327 & 2.74 & $0.0134^{*}$ & 0.1800545 & 1.3579455 \\
C $^{\star} \mathrm{C}$ & -0.5565 & 0.280327 & -1.99 & 0.0626 & -1.145446 & 0.0324455
\end{tabular}




\section{Method 1 - Stress Standard Deviation Model:}

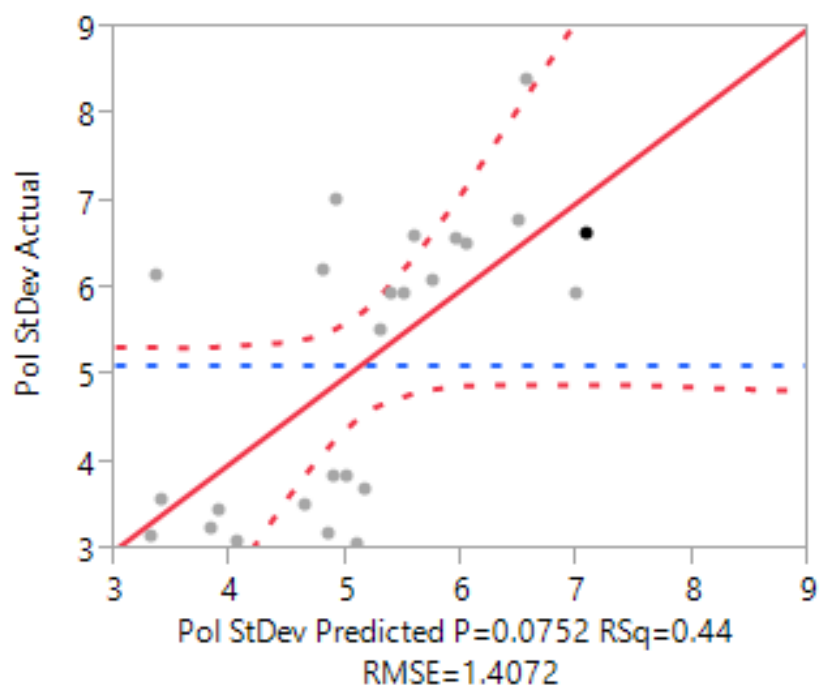

\section{Summary of Fit}

RSquare

0.43872

RSquare Adj

0.251626

Root Mean Square Error

1.407224

Mean of Response

5.11724

Observations (or Sum Wgts)

\section{Analysis of Variance}

\begin{tabular}{lrrrrc} 
& \multicolumn{5}{c}{ Sum of } \\
Source & DF & Squares & Mean Square & F Ratio \\
Model & 6 & 27.861598 & 4.64360 & 2.3449 \\
\hline Error & 18 & 35.645031 & 1.98028 & Prob > F \\
C. Total & 24 & 63.506629 & & 0.0752
\end{tabular}

\section{Parameter Estimates}

$\begin{array}{lrrrrrr}\text { Term } & \text { Estimate } & \text { Std Error } & \text { t Ratio } & \text { Prob> }|\mathbf{t}| & \text { Lower 95\% } & \text { Upper 95\% } \\ \text { Intercept } & 4.906198 & 0.577334 & 8.50 & <.0001^{*} & 3.6932646 & 6.1191314 \\ \text { A } & -0.043556 & 0.331686 & -0.13 & 0.8970 & -0.740402 & 0.6532906 \\ \text { B } & 0.8443889 & 0.331686 & 2.55 & 0.0203^{*} & 0.1475427 & 1.5412351 \\ \text { C } & -0.743389 & 0.331686 & -2.24 & 0.0379^{*} & -1.440235 & -0.046543 \\ \text { D } & 0.2562222 & 0.331686 & 0.77 & 0.4499 & -0.440624 & 0.9530684 \\ \text { A }^{\star} \mathrm{A} & 1.0843069 & 0.785883 & 1.38 & 0.1846 & -0.566772 & 2.7353856 \\ \text { C }^{\star} \mathrm{C} & -0.791193 & 0.785883 & -1.01 & 0.3274 & -2.442272 & 0.8598856\end{array}$


Method $1-90^{\text {th }}$ Percentile Stress Model:

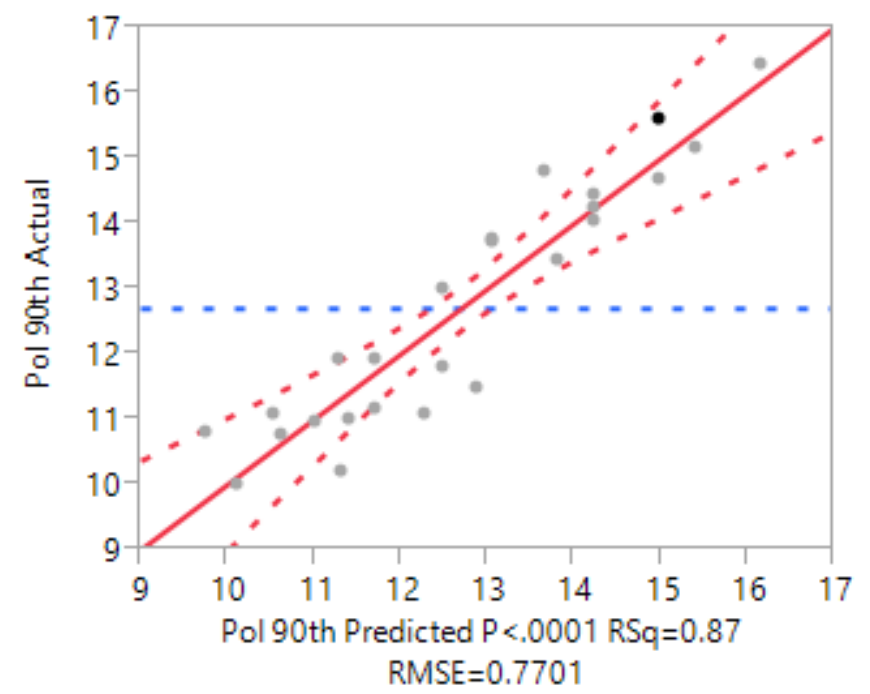

\section{Summary of Fit}

\begin{tabular}{lrrrr}
\hline RSquare & 0.8744 & \\
RSquare Adj & 0.832534 & \\
Root Mean Square Error & 0.770062 & \\
Mean of Response & 12.69668 & \\
Observations (or Sum Wgts) & 25 & \\
\hline \multicolumn{5}{|c}{ Analysis of Variance } \\
\begin{tabular}{l}
\multicolumn{5}{c}{ Sum of } \\
Source
\end{tabular} DF & Squares & Mean Square & F Ratio \\
Model & 6 & 74.309670 & 12.3849 & 20.8854 \\
Error & 18 & 10.673927 & 0.5930 & Prob > F \\
C. Total & 24 & 84.983597 & & $<.0001^{*}$
\end{tabular}

\section{Parameter Estimates}

$\begin{array}{lrrrrrr}\text { Term } & \text { Estimate } & \text { Std Error } & \text { t Ratio } & \text { Prob }>|\mathbf{t}| & \text { Lower 95\% } & \text { Upper 95\% } \\ \text { Intercept } & 11.009446 & 0.315929 & 34.85 & <.0001^{*} & 10.345703 & 11.673188 \\ \text { A } & -0.586167 & 0.181505 & -3.23 & 0.0047^{*} & -0.967495 & -0.204838 \\ \text { B } & 0.3869444 & 0.181505 & 2.13 & 0.0471^{*} & 0.0056157 & 0.7682732 \\ \text { C } & 0.586 & 0.181505 & 3.23 & 0.0047^{*} & 0.2046713 & 0.9673287 \\ \text { D } & 1.2551667 & 0.181505 & 6.92 & <.0001^{*} & 0.8738379 & 1.6364954 \\ \text { A }^{*} \text { A } & -0.303559 & 0.430051 & -0.71 & 0.4893 & -1.207064 & 0.5999452 \\ \text { C }^{*} \mathrm{C} & 2.6469406 & 0.430051 & 6.15 & <.0001^{*} & 1.7434359 & 3.5504452\end{array}$




\section{Method 2 - Mean \% of Intact Stress Model:}

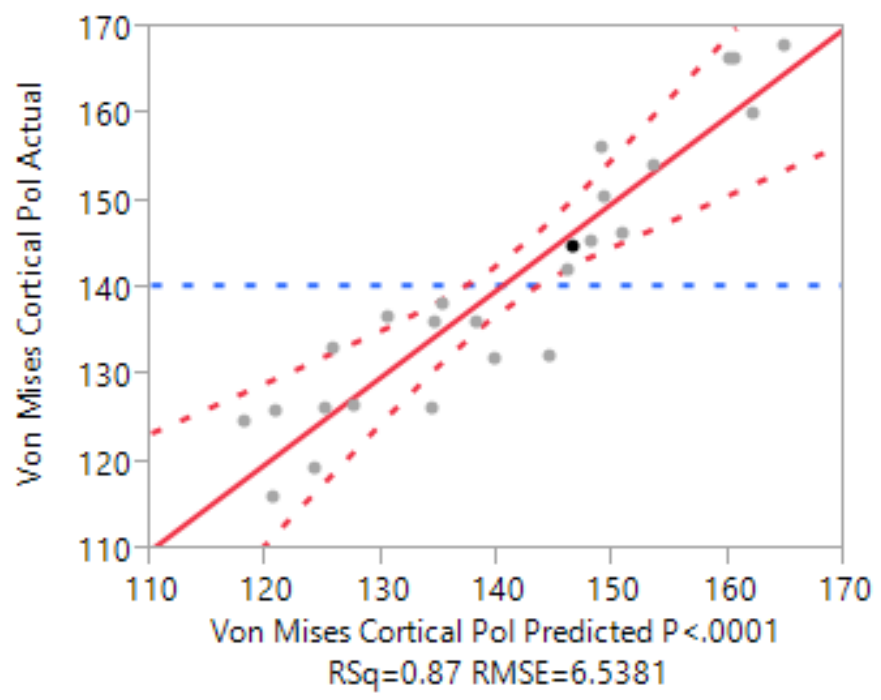

\begin{tabular}{|c|c|c|c|c|}
\hline \multicolumn{4}{|c|}{ Summary of Fit } & \\
\hline \multicolumn{3}{|l|}{ RSquare } & 0.874638 & \\
\hline \multicolumn{3}{|c|}{ RSquare Adj } & 0.811957 & \\
\hline \multicolumn{3}{|c|}{ Root Mean Square Error } & 6.538135 & \\
\hline \multicolumn{3}{|c|}{ Mean of Response } & 140.4032 & \\
\hline \multicolumn{3}{|c|}{ Observations (or Sum Wgts) } & 25 & \\
\hline \multicolumn{5}{|c|}{ Analysis of Variance } \\
\hline Source & DF & $\begin{array}{r}\text { Sum of } \\
\text { Squares }\end{array}$ & Mean Square & F Ratio \\
\hline Model & 8 & 4771.8929 & 596.487 & 13.9538 \\
\hline Error & 16 & 683.9554 & 42.747 & Prob $>\mathrm{F}$ \\
\hline C. Total & 24 & 5455.8484 & & $<.0001^{*}$ \\
\hline
\end{tabular}

\section{Parameter Estimates}

\begin{tabular}{lrrrrrr}
\hline Term & Estimate & Std Error & t Ratio & Prob $>|\mathbf{t}|$ & Lower 95\% & Upper 95\% \\
\hline Intercept & 127.67018 & 2.682364 & 47.60 & $<.0001^{*}$ & 121.98382 & 133.35654 \\
$\mathrm{~A}$ & -6.984 & 1.541053 & -4.53 & $0.0003^{*}$ & -10.25089 & -3.717113 \\
$\mathrm{~B}$ & 3.5087778 & 1.541053 & 2.28 & $0.0369^{*}$ & 0.2418907 & 6.7756649 \\
$\mathrm{C}$ & 2.2661111 & 1.541053 & 1.47 & 0.1608 & -1.000776 & 5.5329982 \\
$\mathrm{D}$ & 6.7857778 & 1.541053 & 4.40 & $0.0004^{*}$ & 3.5188907 & 10.052665 \\
$\mathrm{~B}^{\star} \mathrm{C}$ & 4.29975 & 1.634534 & 2.63 & $0.0182^{*}$ & 0.834693 & 7.764807 \\
$\mathrm{C}^{\star} \mathrm{D}$ & -4.394 & 1.634534 & -2.69 & $0.0162^{*}$ & -7.859057 & -0.928943 \\
$\mathrm{~B}^{\star} \mathrm{B}$ & -6.013624 & 3.651308 & -1.65 & 0.1191 & -13.75405 & 1.7268041 \\
$\mathrm{C}^{\star} \mathrm{C}$ & 23.698376 & 3.651308 & 6.49 & $<.0001^{*}$ & 15.957948 & 31.438804
\end{tabular}




\section{Method 2 - \% of Intact Stress Standard Deviation Model:}

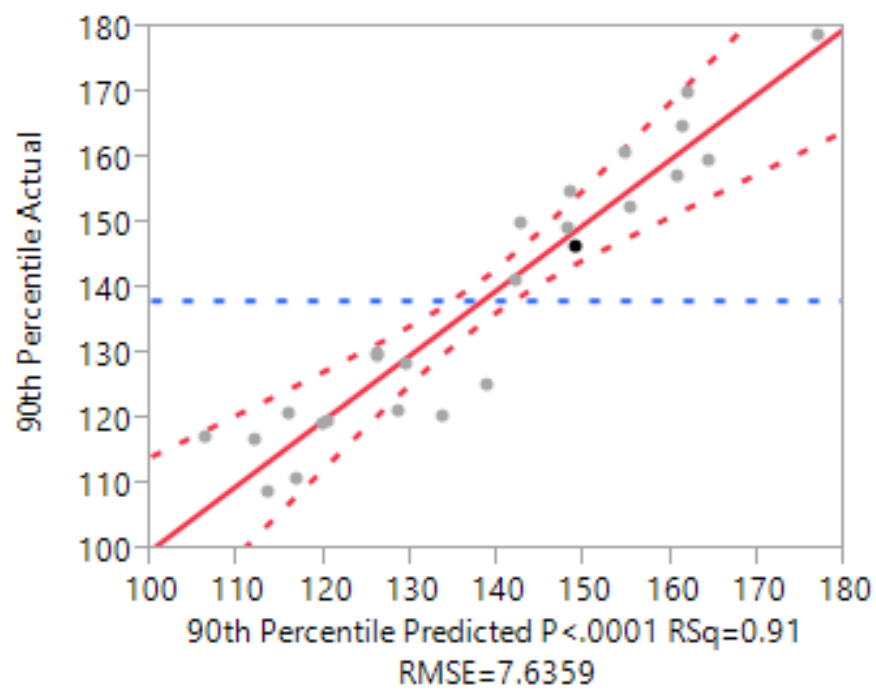

\begin{tabular}{|l|r|}
\hline Summary of Fit & \\
\hline RSquare & 0.907275 \\
RSquare Adj & 0.860913 \\
Root Mean Square Error & 7.635869 \\
Mean of Response & 138.1478 \\
\hline Observations (or Sum Wgts) & 25 \\
\hline
\end{tabular}

\begin{tabular}{|l|r|rrr|}
\hline \multicolumn{5}{l|}{ Analysis of Variance } \\
\hline Source & DF & Sum of & & \\
Squares & Mean Square & F Ratio \\
Model & 8 & 9128.101 & 1141.01 & 19.5692 \\
\hline Error & 16 & 932.904 & 58.31 & Prob > F \\
C. Total & 24 & 10061.005 & & $<.0001^{*}$
\end{tabular}

\section{Parameter Estimates}

$\begin{array}{lrrrrrr}\text { Term } & \text { Estimate } & \text { Std Error } & \text { t Ratio } & \text { Prob }>|\mathbf{t}| & \text { Lower 95\% } & \text { Upper 95\% } \\ \text { Intercept } & 119.93304 & 3.132725 & 38.28 & <.0001^{*} & 113.29196 & 126.57412 \\ \text { A } & -6.379056 & 1.799792 & -3.54 & 0.0027^{\star} & -10.19444 & -2.563668 \\ \text { B } & 4.2104444 & 1.799792 & 2.34 & 0.0326^{\star} & 0.3950566 & 8.0258323 \\ \text { C } & 6.3755 & 1.799792 & 3.54 & 0.0027^{\star} & 2.5601122 & 10.190888 \\ \text { D } & 13.657333 & 1.799792 & 7.59 & <.0001^{\star} & 9.8419455 & 17.472721 \\ B^{\star} \mathrm{C} & 3.711625 & 1.908967 & 1.94 & 0.0697 & -0.335205 & 7.7584549 \\ \text { C }^{\star} \mathrm{D} & -2.489375 & 1.908967 & -1.30 & 0.2107 & -6.536205 & 1.5574549 \\ \mathrm{~B}^{\star} \mathrm{B} & -3.800139 & 4.264352 & -0.89 & 0.3861 & -12.84016 & 5.2398843 \\ \mathrm{C}^{\star} \mathrm{C} & 29.098361 & 4.264352 & 6.82 & <.0001^{*} & 20.058338 & 38.138384\end{array}$




\section{Method $2-90^{\text {th }}$ Percentile \% of Intact Stress Model:}

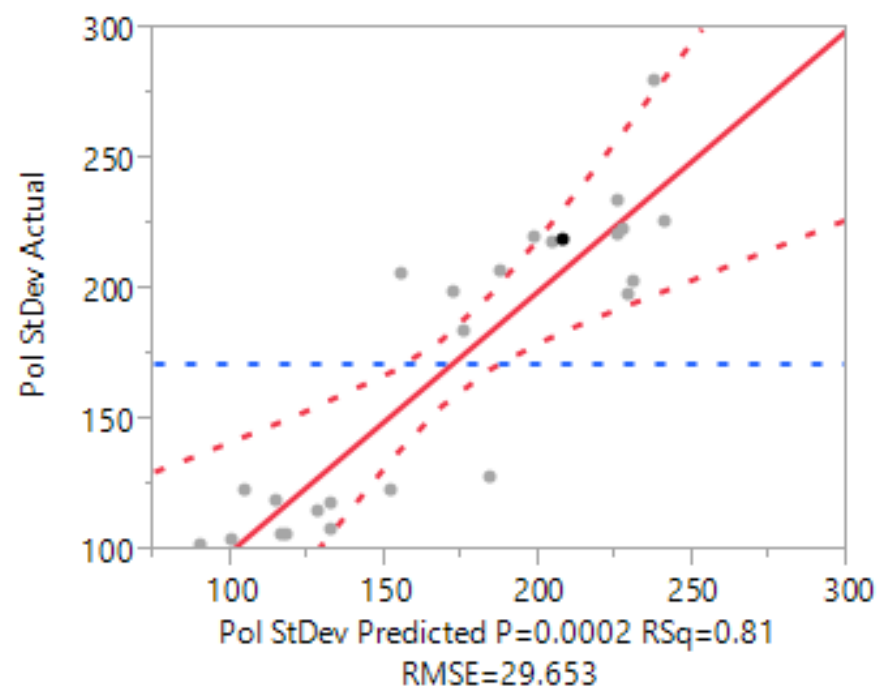

\section{Summary of Fit}

\begin{tabular}{|c|c|c|c|c|}
\hline \multicolumn{3}{|l|}{ RSquare } & \multirow{2}{*}{\multicolumn{2}{|c|}{0.807652}} \\
\hline \multicolumn{3}{|c|}{ RSquare Adj } & & \\
\hline \multicolumn{3}{|c|}{ Root Mean Square Error } & 29.65338 & \\
\hline \multicolumn{3}{|c|}{ Mean of Response } & 171.6625 & \\
\hline \multicolumn{3}{|c|}{ Observations (or Sum Wgts) } & 25 & \\
\hline \multicolumn{5}{|c|}{ Analysis of Variance } \\
\hline Source & DF & $\begin{array}{l}\text { Sum of } \\
\text { Squares }\end{array}$ & Mean Square & F Ratio \\
\hline Model & 8 & 59075.304 & 7384.41 & 8.3978 \\
\hline Error & 16 & 14069.170 & 879.32 & Prob $>F$ \\
\hline C. Total & 24 & 73144.474 & & $0.0002^{*}$ \\
\hline
\end{tabular}

\section{Parameter Estimates}

$\begin{array}{lrrrrrr}\text { Term } & \text { Estimate } & \text { Std Error } & \text { t Ratio } & \text { Prob }>|\mathbf{t}| & \text { Lower 95\% } & \text { Upper 95\% } \\ \text { Intercept } & 116.5055 & 12.16573 & 9.58 & <.0001^{*} & 90.715318 & 142.29569 \\ \text { A } & 1.7622222 & 6.989369 & 0.25 & 0.8042 & -13.05458 & 16.579024 \\ \text { B } & 7.4430556 & 6.989369 & 1.06 & 0.3027 & -7.373746 & 22.259857 \\ \text { C } & 13.424667 & 6.989369 & 1.92 & 0.0728 & -1.392135 & 28.241468 \\ \text { D } & 15.881389 & 6.989369 & 2.27 & 0.0372^{*} & 1.0645875 & 30.69819 \\ B^{\star} \mathrm{C} & 18.946063 & 7.413346 & 2.56 & 0.0212^{\star} & 3.2304714 & 34.661654 \\ \mathrm{C}^{\star} \mathrm{D} & -21.85656 & 7.413346 & -2.95 & 0.0094^{*} & -37.57215 & -6.140971 \\ \mathrm{~B}^{\star} \mathrm{B} & -19.01677 & 16.56032 & -1.15 & 0.2677 & -54.12309 & 16.089552 \\ \mathrm{C}^{\star} \mathrm{C} & 95.623733 & 16.56032 & 5.77 & <.0001^{*} & 60.517413 & 130.73005\end{array}$




\section{Approach 2 Outcomes - All Regions Mean Stress}

\section{Method 1 - Proximal Region Stress:}

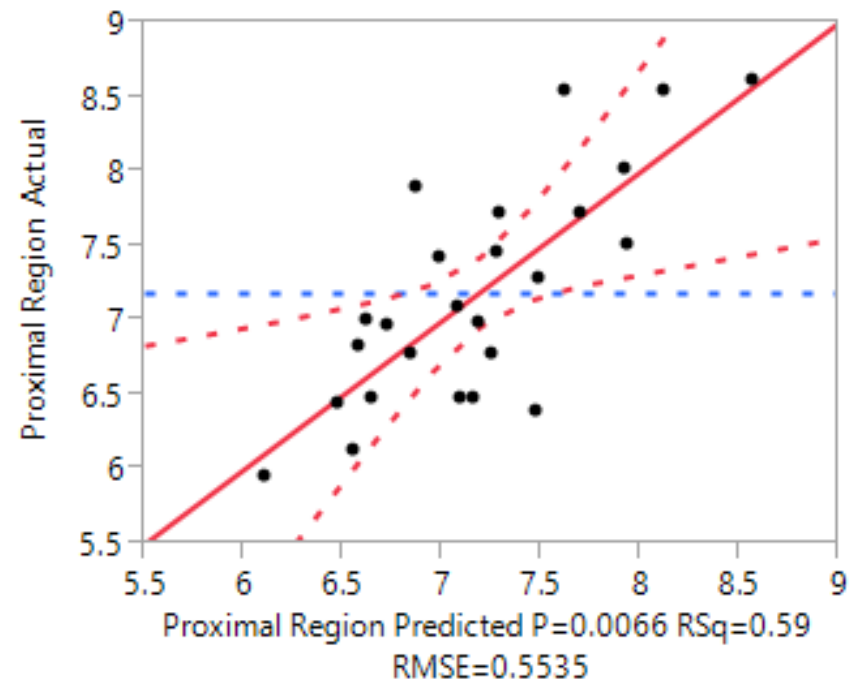

\begin{tabular}{l|r|}
\hline Summary of Fit & \\
\hline RSquare & 0.594108 \\
RSquare Adj & 0.458811 \\
Root Mean Square Error & 0.553522 \\
Mean of Response & 7.181 \\
Observations (or Sum Wgts) & 25
\end{tabular}

\section{Analysis of Variance}

\begin{tabular}{lrrrrc} 
& \multicolumn{5}{c}{ Sum of } \\
Source & DF & Squares & Mean Square & F Ratio \\
Model & 6 & 8.072317 & 1.34539 & 4.3911 \\
\hline Error & 18 & 5.514963 & 0.30639 & Prob > F \\
C. Total & 24 & 13.587280 & & $0.0066^{*}$
\end{tabular}

\section{Parameter Estimates}

$\begin{array}{lrrrrrr}\text { Term } & \text { Estimate } & \text { Std Error } & \text { t Ratio } & \text { Prob> }|\mathbf{t}| & \text { Lower 95\% } & \text { Upper 95\% } \\ \text { Intercept } & 6.869 & 0.209212 & 32.83 & <.0001^{*} & 6.4294625 & 7.3085375 \\ \text { A } & -0.314444 & 0.130466 & -2.41 & 0.0269^{*} & -0.588544 & -0.040345 \\ \text { B } & 0.4008333 & 0.130466 & 3.07 & 0.0066^{*} & 0.1267335 & 0.6749332 \\ \text { C } & -0.2915 & 0.130466 & -2.23 & 0.0384^{*} & -0.5656 & -0.0174 \\ \text { D } & 0.225 & 0.130466 & 1.72 & 0.1017 & -0.0491 & 0.4990998 \\ \text { B }^{\star} \text { C } & -0.028938 & 0.138381 & -0.21 & 0.8367 & -0.319664 & 0.2617893 \\ \text { A A }^{*} & 0.4333333 & 0.246558 & 1.76 & 0.0958 & -0.084667 & 0.9513333\end{array}$




\section{Method 1 - Inferior Region Stress:}

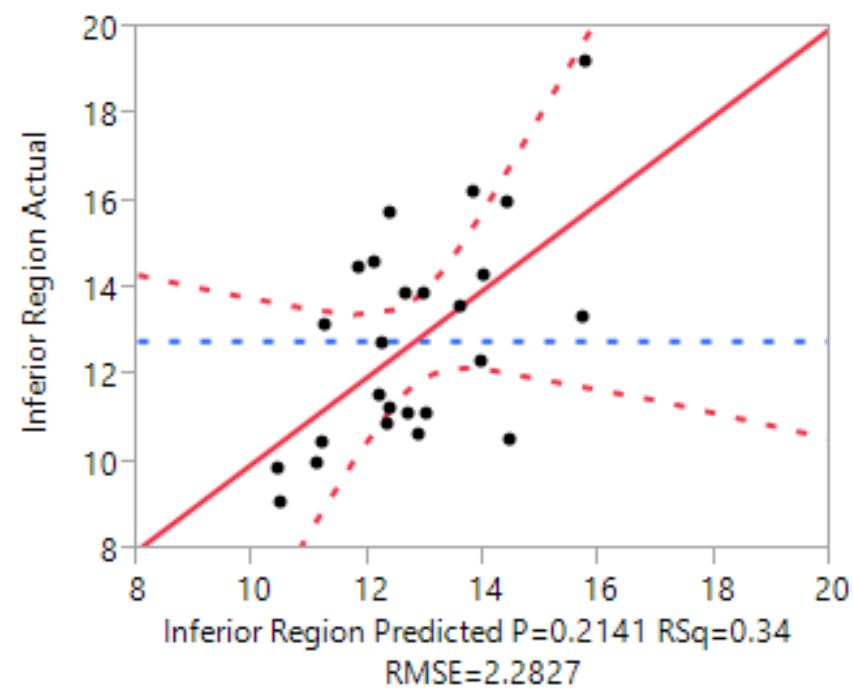

\section{Summary of Fit}

\begin{tabular}{l|r}
\hline RSquare & 0.342931 \\
RSquare Adj & 0.123908 \\
Root Mean Square Error & 2.282704 \\
Mean of Response & 12.79168 \\
\hline Observations (or Sum Wgts) & 25
\end{tabular}

\section{Analysis of Variance}

\begin{tabular}{|c|c|c|c|c|}
\hline Source & DF & $\begin{array}{r}\text { Sum of } \\
\text { Squares }\end{array}$ & Mean Square & F Ratio \\
\hline Model & 6 & 48.95158 & 8.15860 & 1.5657 \\
\hline Error & 18 & 93.79324 & 5.21074 & Prob $>F$ \\
\hline C. Total & 24 & 142.74482 & & 0.2141 \\
\hline
\end{tabular}

\section{Parameter Estimates}

$\begin{array}{lrrrrrr}\text { Term } & \text { Estimate } & \text { Std Error } & \text { t Ratio } & \text { Prob> }|\mathbf{t}| & \text { Lower 95\% } & \text { Upper 95\% } \\ \text { Intercept } & 12.354429 & 0.862781 & 14.32 & <.0001^{*} & 10.541793 & 14.167064 \\ \text { A } & -0.877722 & 0.538038 & -1.63 & 0.1202 & -2.008099 & 0.2526545 \\ \text { B } & 1.2486667 & 0.538038 & 2.32 & 0.0322^{*} & 0.11829 & 2.3790434 \\ \text { C } & -0.517389 & 0.538038 & -0.96 & 0.3490 & -1.647766 & 0.6129878 \\ \text { D } & -0.022333 & 0.538038 & -0.04 & 0.9673 & -1.15271 & 1.1080434 \\ \text { B }^{\star} \mathrm{C} & -0.144313 & 0.570676 & -0.25 & 0.8032 & -1.343258 & 1.054633 \\ \text { A }^{\star} \mathrm{A} & 0.6072937 & 1.016797 & 0.60 & 0.5578 & -1.528918 & 2.7435048\end{array}$




\section{Method 1 - Superior Region Stress:}

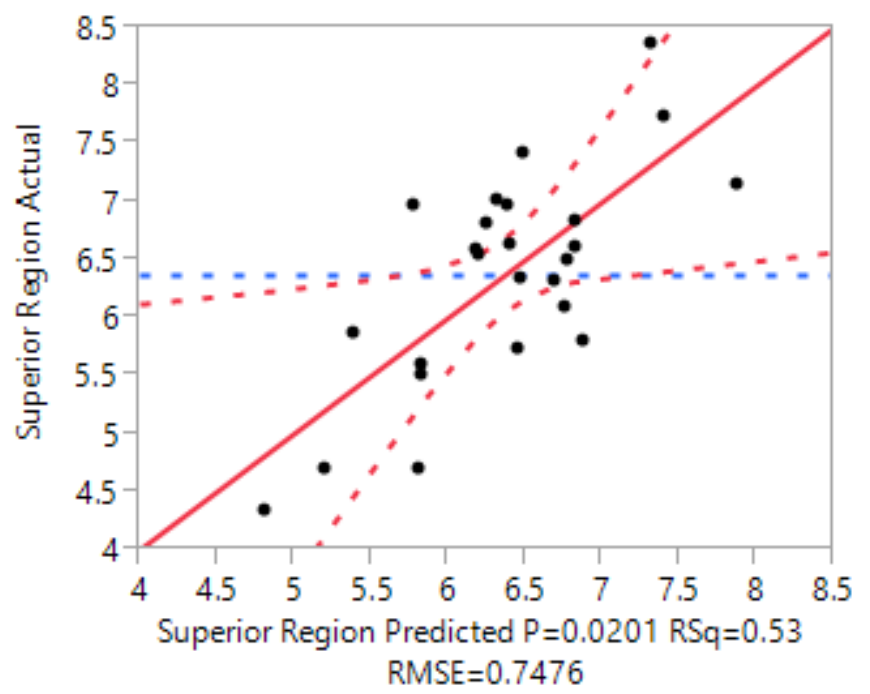

\section{Summary of Fit}

\begin{tabular}{l|r}
\hline RSquare & 0.531412 \\
\hline RSquare Adj & 0.375216 \\
\hline Root Mean Square Error & 0.7476 \\
\hline Mean of Response & 6.36488 \\
\hline Observations (or Sum Wgts) & 25
\end{tabular}

\section{Analysis of Variance}

\begin{tabular}{|c|c|c|c|c|}
\hline Source & DF & $\begin{array}{r}\text { Sum of } \\
\text { Squares }\end{array}$ & Mean Square & F Ratio \\
\hline Model & 6 & 11.409098 & 1.90152 & 3.4022 \\
\hline Error & 18 & 10.060307 & 0.55891 & Prob $>F$ \\
\hline C. Total & 24 & 21.469405 & & $0.0201^{*}$ \\
\hline
\end{tabular}

\section{Parameter Estimates}

$\begin{array}{lrrrrrrr}\text { Term } & \text { Estimate } & \text { Std Error } & \text { t Ratio } & \text { Prob> } \mathbf{| t |} & \text { Lower 95\% } & \text { Upper 95\% } \\ \text { Intercept } & 6.47 & 0.282566 & 22.90 & <.0001^{*} & 5.8763503 & 7.0636497 \\ \text { A } & -0.498889 & 0.176211 & -2.83 & 0.0111^{*} & -0.869095 & -0.128683 \\ \text { B } & -0.023389 & 0.176211 & -0.13 & 0.8959 & -0.393595 & 0.3468168 \\ \text { C } & 0.2196111 & 0.176211 & 1.25 & 0.2286 & -0.150595 & 0.5898168 \\ \text { D } & -0.284167 & 0.176211 & -1.61 & 0.1242 & -0.654372 & 0.086039 \\ \text { B }^{\star} \text { C } & -0.52975 & 0.1869 & -2.83 & 0.0110^{*} & -0.922412 & -0.137088 \\ \text { A }^{*} \mathrm{~A} & -0.146 & 0.333008 & -0.44 & 0.6663 & -0.845623 & 0.5536229\end{array}$




\section{Method 1 - Lateral Region Stress:}

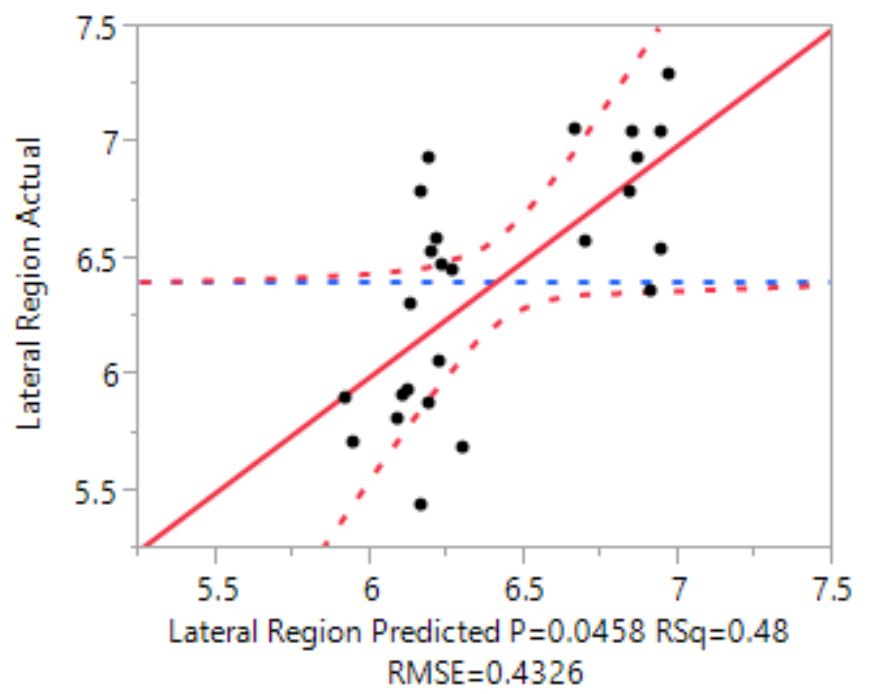

\begin{tabular}{|c|c|c|c|c|}
\hline \multicolumn{4}{|c|}{ Summary of Fit } & \\
\hline \multicolumn{3}{|c|}{ RSquare } & 0.476553 & \\
\hline \multicolumn{3}{|c|}{ RSquare Adj } & 0.302071 & \\
\hline \multicolumn{3}{|c|}{ Root Mean Square Error } & 0.432613 & \\
\hline \multicolumn{3}{|c|}{ Mean of Response } & 6.40452 & \\
\hline \multicolumn{3}{|c|}{ Observations (or Sum Wgts) } & 25 & \\
\hline \multicolumn{5}{|c|}{ Analysis of Variance } \\
\hline \multirow{2}{*}{$\begin{array}{l}\text { Source } \\
\text { Model }\end{array}$} & DF & $\begin{array}{l}\text { Sum of } \\
\text { Squares }\end{array}$ & Mean Square & F Ratio \\
\hline & 6 & 3.0669816 & 0.511164 & 2.7312 \\
\hline Error & 18 & 3.3687766 & 0.187154 & Prob $>F$ \\
\hline C. Total & 24 & 6.4357582 & & $0.0458^{*}$ \\
\hline
\end{tabular}

\section{Parameter Estimates}

\begin{tabular}{lrrrrrr} 
Term & Estimate & Std Error & t Ratio & Prob $>|\mathbf{t}|$ & Lower 95\% & Upper 95\% \\
\hline Intercept & 6.2155714 & 0.163512 & 38.01 & $<.0001^{*}$ & 5.8720445 & 6.5590983 \\
A & -0.373778 & 0.101968 & -3.67 & $0.0018^{*}$ & -0.588004 & -0.159551 \\
B & 0.0869444 & 0.101968 & 0.85 & 0.4050 & -0.127282 & 0.3011711 \\
C & -0.049722 & 0.101968 & -0.49 & 0.6317 & -0.263949 & 0.1645044 \\
D & 0.0141111 & 0.101968 & 0.14 & 0.8915 & -0.200116 & 0.2283378 \\
$B^{\star}$ C & 0.0361875 & 0.108153 & 0.33 & 0.7418 & -0.191034 & 0.2634092 \\
A A $^{*}$ & 0.2624286 & 0.192701 & 1.36 & 0.1901 & -0.142422 & 0.6672789
\end{tabular}




\section{Method 2 - Proximal Region \% of Intact:}

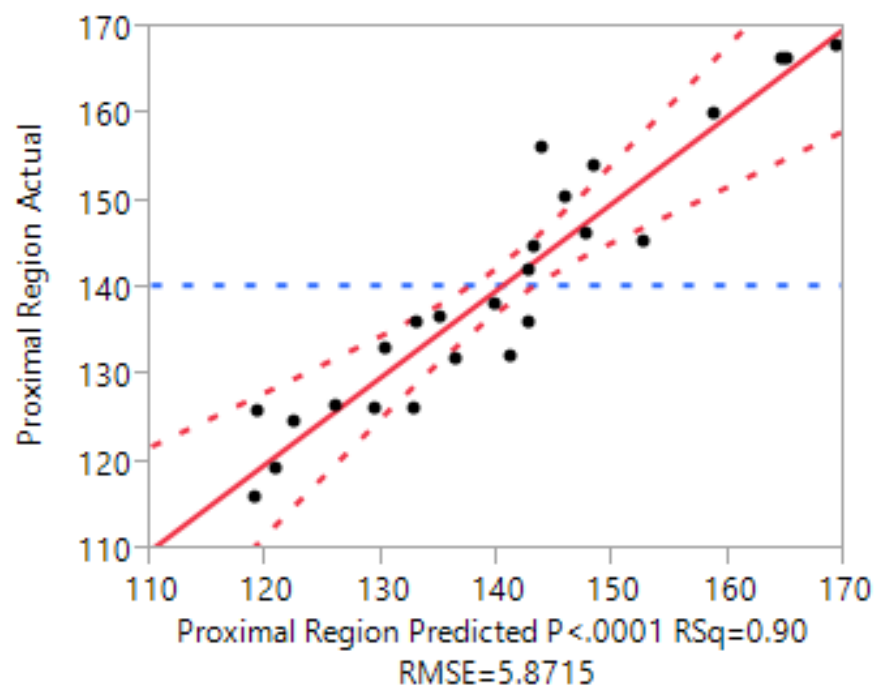

\begin{tabular}{|c|c|c|c|c|}
\hline \multicolumn{4}{|c|}{ Summary of Fit } & \\
\hline \multicolumn{3}{|c|}{ RSquare } & 0.898899 & \\
\hline \multicolumn{3}{|c|}{ RSquare Adj } & 0.848348 & \\
\hline \multicolumn{3}{|c|}{ Root Mean Square Error } & 5.871511 & \\
\hline \multicolumn{3}{|c|}{ Mean of Response } & 140.4032 & \\
\hline \multicolumn{3}{|c|}{ Observations (or Sum Wgts) } & 25 & \\
\hline \multicolumn{5}{|c|}{ Analysis of Variance } \\
\hline Source & DF & $\begin{array}{r}\text { Sum of } \\
\text { Squares }\end{array}$ & Mean Square & F Ratio \\
\hline Model & 8 & 4904.2541 & 613.032 & 17.7821 \\
\hline Error & 16 & 551.5942 & 34.475 & Prob $>F$ \\
\hline C. Total & 24 & 5455.8484 & & $<.0001^{*}$ \\
\hline
\end{tabular}

\section{Parameter Estimates}

\begin{tabular}{lrrrrrrr}
\hline Term & Estimate & Std Error & t Ratio & Prob $>|\mathbf{t}|$ & Lower 95\% & Upper 95\% \\
\hline Intercept & 125.952 & 2.219223 & 56.76 & $<.0001^{*}$ & 121.24746 & 130.65654 \\
\hline $\mathrm{A}$ & -6.984 & 1.383928 & -5.05 & $0.0001^{*}$ & -9.917797 & -4.050203 \\
$\mathrm{~B}$ & 3.5087778 & 1.383928 & 2.54 & $0.0220^{*}$ & 0.5749807 & 6.4425749 \\
$\mathrm{C}$ & 2.2661111 & 1.383928 & 1.64 & 0.1211 & -0.667686 & 5.1999082 \\
$\mathrm{D}$ & 6.7857778 & 1.383928 & 4.90 & $0.0002^{*}$ & 3.8519807 & 9.7195749 \\
$\mathrm{~A}^{\star} \mathrm{B}$ & -3.9395 & 1.467878 & -2.68 & $0.0163^{*}$ & -7.051262 & -0.827738 \\
$\mathrm{~B}^{\star} \mathrm{C}$ & 4.29975 & 1.467878 & 2.93 & $0.0098^{*}$ & 1.1879882 & 7.4115118 \\
$\mathrm{C}^{\star} \mathrm{D}$ & -4.394 & 1.467878 & -2.99 & $0.0086^{*}$ & -7.505762 & -1.282238 \\
$\mathrm{C}^{\star} \mathrm{C}$ & 20.071111 & 2.615379 & 7.67 & $<.0001^{*}$ & 14.526756 & 25.615467
\end{tabular}




\section{Method 2 - Inferior Region \% of Intact:}

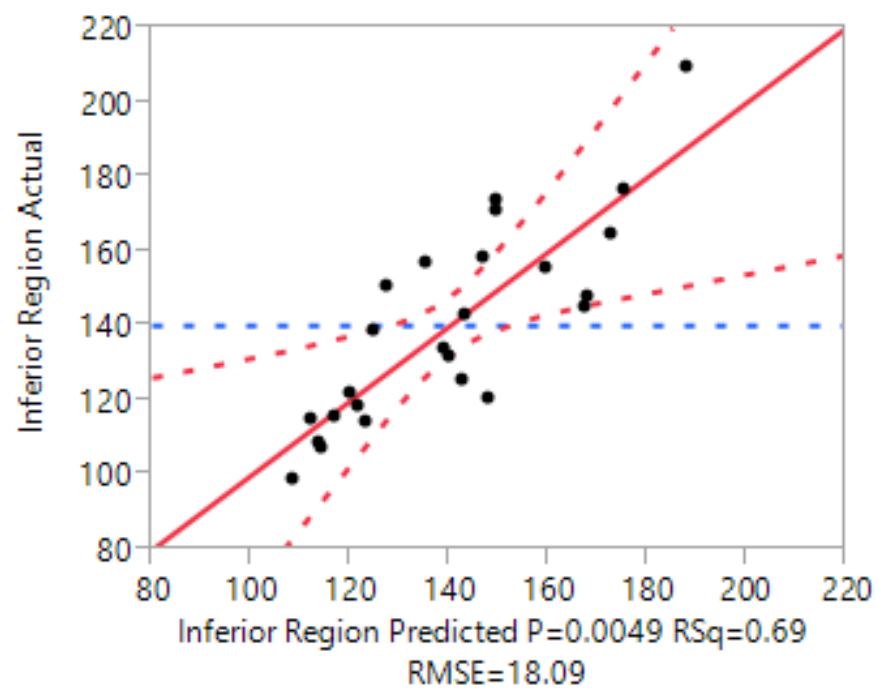

\section{Summary of Fit}

\begin{tabular}{l|r}
\hline RSquare & 0.694296 \\
RSquare Adj & 0.541444 \\
Root Mean Square Error & 18.0902 \\
Mean of Response & 140.193 \\
Observations (or Sum Wgts) & 25
\end{tabular}

\section{Analysis of Variance}

\begin{tabular}{|c|c|c|c|c|}
\hline Source & DF & $\begin{array}{l}\text { Sum of } \\
\text { Squares }\end{array}$ & Mean Square & F Ratio \\
\hline Model & 8 & 11891.868 & 1486.48 & 4.5423 \\
\hline Error & 16 & 5236.083 & 327.26 & Prob $>F$ \\
\hline C. Total & 24 & 17127.951 & & $0.0049 *$ \\
\hline
\end{tabular}

\section{Parameter Estimates}

$\begin{array}{lrrrrrr}\text { Term } & \text { Estimate } & \text { Std Error } & \text { t Ratio } & \text { Prob }>|\mathbf{t}| & \text { Lower 95\% } & \text { Upper 95\% } \\ \text { Intercept } & 116.692 & 6.837451 & 17.07 & <.0001^{*} & 102.19725 & 131.18675 \\ \text { A } & -8.162944 & 4.2639 & -1.91 & 0.0736 & -17.20201 & 0.8761195 \\ \text { B } & 4.7283889 & 4.2639 & 1.11 & 0.2838 & -4.310675 & 13.767453 \\ \text { C } & 0.0414444 & 4.2639 & 0.01 & 0.9924 & -8.99762 & 9.0805084 \\ \text { D } & 3.0672222 & 4.2639 & 0.72 & 0.4823 & -5.971842 & 12.106286 \\ A^{\star} \mathrm{B} & -5.93325 & 4.522549 & -1.31 & 0.2081 & -15.52063 & 3.6541251 \\ B^{\star} \mathrm{C} & 9.318125 & 4.522549 & 2.06 & 0.0560 & -0.26925 & 18.9055 \\ \mathrm{C}^{\star} \mathrm{D} & -13.2255 & 4.522549 & -2.92 & 0.0099^{\star} & -22.81288 & -3.638125 \\ \mathrm{C}^{\star} \mathrm{C} & 32.640222 & 8.058013 & 4.05 & 0.0009^{*} & 15.557997 & 49.722447\end{array}$




\section{Method 2 - Superior Region \% of Intact:}

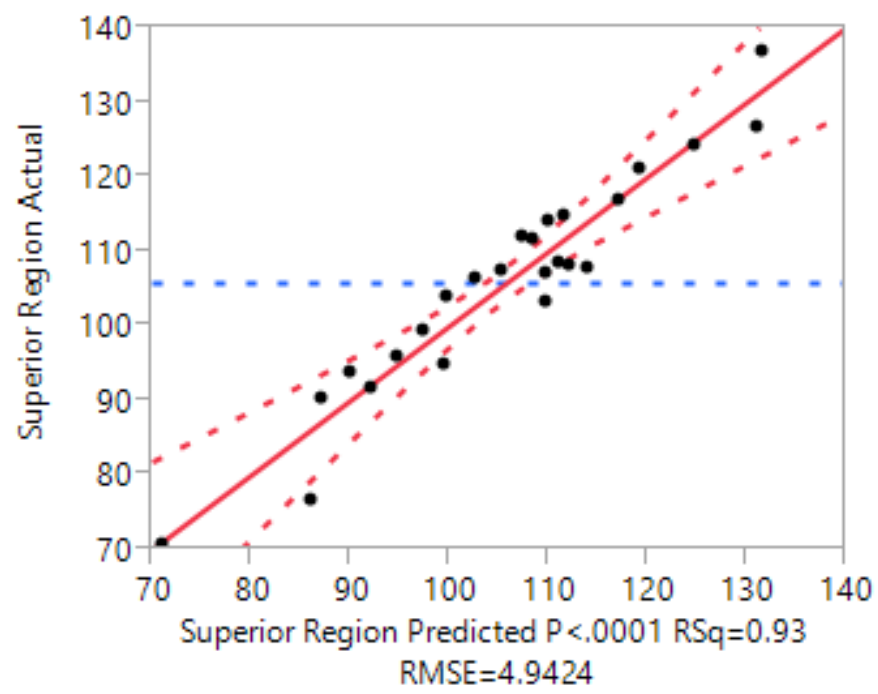

\section{Summary of Fit}

\begin{tabular}{lr} 
RSquare & 0.925494 \\
RSquare Adj & 0.888242 \\
Root Mean Square Error & 4.942392 \\
Mean of Response & 105.7401 \\
Observations (or Sum Wgts) & 25 \\
\hline
\end{tabular}

\section{Analysis of Variance}

\begin{tabular}{|c|c|c|c|c|}
\hline Source & DF & $\begin{array}{l}\text { Sum of } \\
\text { Squares }\end{array}$ & Mean Square & F Ratio \\
\hline Model & 8 & 4854.8861 & 606.861 & 24.8436 \\
\hline Error & 16 & 390.8359 & 24.427 & Prob $>F$ \\
\hline C. Total & 24 & 5245.7220 & & $<, 0001^{*}$ \\
\hline
\end{tabular}

\section{Parameter Estimates}

$\begin{array}{lrrrrrr}\text { Term } & \text { Estimate } & \text { Std Error } & \text { t Ratio } & \text { Prob }>|\mathbf{t}| & \text { Lower 95\% } & \text { Upper 95\% } \\ \text { Intercept } & 108.292 & 1.868049 & 57.97 & <.0001^{*} & 104.33191 & 112.25209 \\ \text { A } & -8.808056 & 1.164933 & -7.56 & <.0001^{*} & -11.2776 & -6.338508 \\ \text { B } & 5.7685556 & 1.164933 & 4.95 & 0.0001^{*} & 3.2990078 & 8.2381033 \\ \text { C } & -4.919111 & 1.164933 & -4.22 & 0.0006^{*} & -7.388659 & -2.449563 \\ \text { D } & -10.84333 & 1.164933 & -9.31 & <.0001^{*} & -13.31288 & -8.373786 \\ A^{\star} \mathrm{B} & -1.242125 & 1.235598 & -1.01 & 0.3297 & -3.861476 & 1.3772259 \\ \mathrm{~B}^{\star} \mathrm{C} & 3.544625 & 1.235598 & 2.87 & 0.0111^{*} & 0.9252741 & 6.1639759 \\ \mathrm{C}^{\star} \mathrm{D} & -1.073875 & 1.235598 & -0.87 & 0.3976 & -3.693226 & 1.5454759 \\ \mathrm{C}^{\star} \mathrm{C} & -3.544333 & 2.201517 & -1.61 & 0.1270 & -8.21134 & 1.1226732\end{array}$




\section{Method 2 - Lateral Region \% of Intact:}

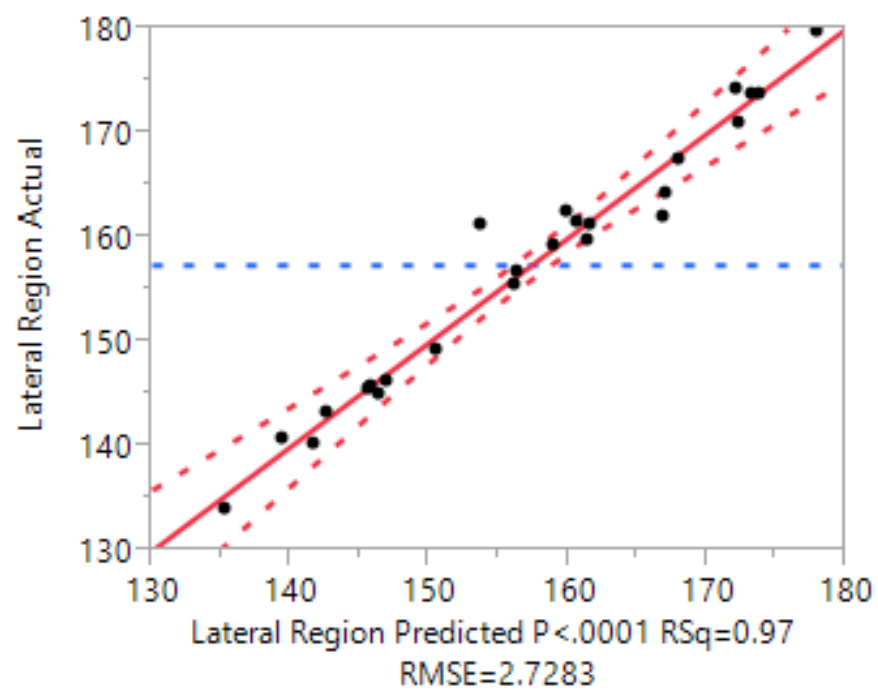

\section{Summary of Fit}

\begin{tabular}{l|r}
\hline RSquare & 0.967758 \\
RSquare Adj & 0.951638 \\
Root Mean Square Error & 2.728333 \\
Mean of Response & 157.3572 \\
Observations (or Sum Wgts) & 25
\end{tabular}

\section{Analysis of Variance}

\begin{tabular}{|c|c|c|c|c|}
\hline Source & DF & $\begin{array}{r}\text { Sum of } \\
\text { Squares }\end{array}$ & Mean Square & F Ratio \\
\hline Model & 8 & 3574.9084 & 446.864 & 60.0316 \\
\hline Error & 16 & 119.1008 & 7.444 & Prob $>F$ \\
\hline C. Total & 24 & 3694.0092 & & $<.0001^{*}$ \\
\hline
\end{tabular}

\begin{tabular}{|c|c|c|c|c|c|c|}
\hline \multicolumn{7}{|c|}{ Parameter Estimates } \\
\hline Term & Estimate & Std Error & t Ratio & Prob $>|t|$ & Lower $95 \%$ & Upper $95 \%$ \\
\hline Intercept & 158.85629 & 1.031213 & 154.05 & $<.0001^{*}$ & 156.67021 & 161.04236 \\
\hline A & -13.20617 & 0.643074 & -20.54 & $<.0001^{*}$ & -14.56942 & -11.84291 \\
\hline B & 2.5018889 & 0.643074 & 3.89 & $0.0013^{\star}$ & 1.1386322 & 3.8651455 \\
\hline C & 3.0302222 & 0.643074 & 4.71 & $0.0002^{*}$ & 1.6669656 & 4.3934789 \\
\hline D & 2.6492222 & 0.643074 & 4.12 & $0.0008^{*}$ & 1.2859656 & 4.0124789 \\
\hline$A^{*} B$ & -0.512 & 0.682083 & -0.75 & 0.4638 & -1.957952 & 0.933952 \\
\hline $\mathrm{B}^{\star} \mathrm{C}$ & -0.14775 & 0.682083 & -0.22 & 0.8312 & -1.593702 & 1.298202 \\
\hline$C^{*} \mathrm{D}$ & -0.558125 & 0.682083 & -0.82 & 0.4252 & -2.004077 & 0.887827 \\
\hline $\mathrm{C}^{\star} \mathrm{C}$ & -2.082063 & 1.215296 & -1.71 & 0.1060 & -4.658376 & 0.4942494 \\
\hline
\end{tabular}




\section{Approach 3 Outcomes - All Regions $90^{\text {th }}$ Percentile}

\section{Method 1 - Proximal Region Stress:}

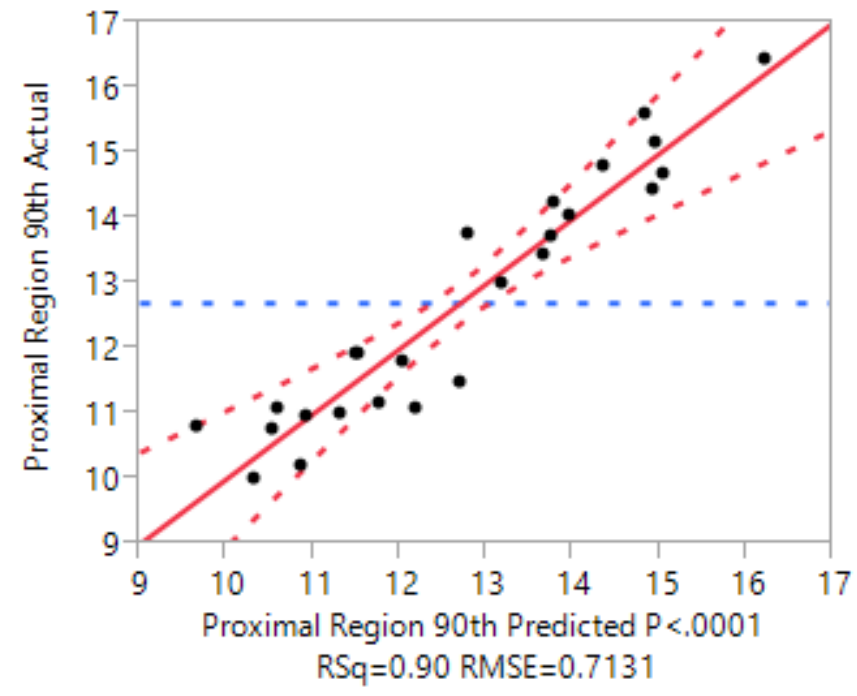

\begin{tabular}{|l|r|}
\hline Summary of Fit & \\
\hline RSquare & 0.904268 \\
RSquare Adj & 0.856402 \\
Root Mean Square Error & 0.713077 \\
Mean of Response & 12.69668 \\
\hline Observations (or Sum Wgts) & 25 \\
\hline
\end{tabular}

Analysis of Variance

\begin{tabular}{|c|c|c|c|c|}
\hline Source & DF & $\begin{array}{l}\text { Sum of } \\
\text { Squares }\end{array}$ & Mean Square & F Ratio \\
\hline Model & 8 & 76.847944 & 9.60599 & 18.8916 \\
\hline Error & 16 & 8.135654 & 0.50848 & Prob $>F$ \\
\hline C. Total & 24 & 84.983597 & & $<.0001^{*}$ \\
\hline
\end{tabular}

\begin{tabular}{lrrrrrrr|}
\hline \multicolumn{6}{l}{ Parameter Estimates } \\
\hline Term & Estimate & Std Error & t Ratio & Prob $>|\mathbf{t}|$ & Lower 95\% & Upper 95\% \\
Intercept & 10.922714 & 0.269518 & 40.53 & $<.0001^{*}$ & 10.351362 & 11.494066 \\
A & -0.586167 & 0.168074 & -3.49 & $0.0030^{*}$ & -0.942467 & -0.229866 \\
B & 0.3869444 & 0.168074 & 2.30 & $0.0351^{*}$ & 0.0306439 & 0.743245 \\
C & 0.586 & 0.168074 & 3.49 & $0.0030^{*}$ & 0.2296995 & 0.9423005 \\
D & 1.2551667 & 0.168074 & 7.47 & $<.0001^{*}$ & 0.8988662 & 1.6114672 \\
$B^{\star} \mathrm{C}$ & 0.3410625 & 0.178269 & 1.91 & 0.0738 & -0.036851 & 0.7189763 \\
$B^{\star} \mathrm{D}$ & -0.091813 & 0.178269 & -0.52 & 0.6136 & -0.469726 & 0.2861013 \\
$\mathrm{C}^{\star} \mathrm{D}$ & -0.228812 & 0.178269 & -1.28 & 0.2176 & -0.606726 & 0.1491013 \\
$\mathrm{C}^{\star} \mathrm{C}$ & 2.4638413 & 0.31763 & 7.76 & $<.0001^{*}$ & 1.7904966 & 3.1371859
\end{tabular}




\section{Method 1 - Inferior Region Stress:}

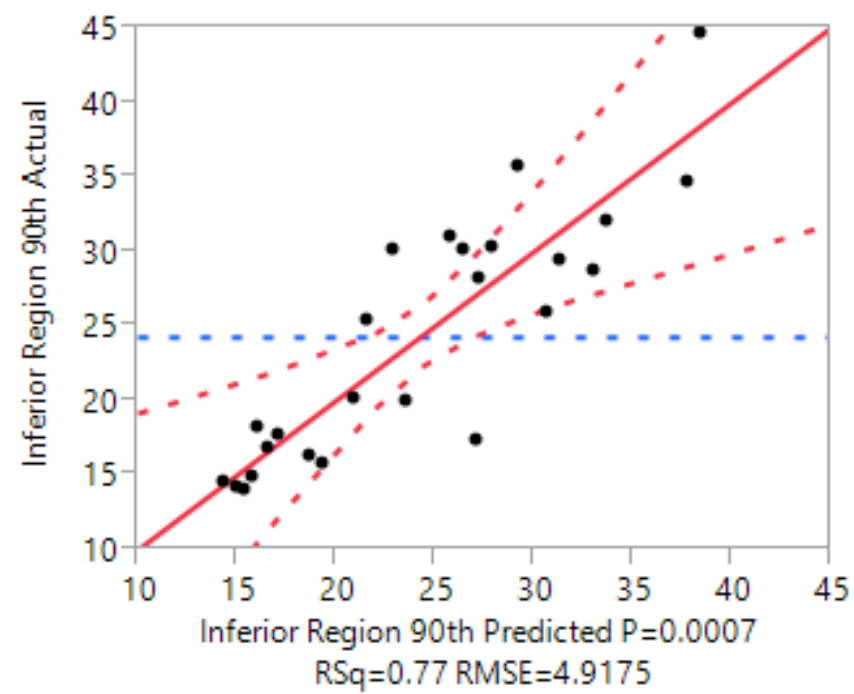

\section{Summary of Fit}

\begin{tabular}{lr}
\hline RSquare & 0.768869 \\
RSquare Adj & 0.653304 \\
Root Mean Square Error & 4.917527 \\
Mean of Response & 24.24108 \\
\hline Observations (or Sum Wgts) & 25
\end{tabular}

\section{Analysis of Variance}

\begin{tabular}{|c|c|c|c|c|}
\hline Source & DF & $\begin{array}{l}\text { Sum of } \\
\text { Squares }\end{array}$ & Mean Square & F Ratio \\
\hline lodel & 8 & 1287.0897 & 160.886 & 6.6531 \\
\hline ror & 16 & 386.9131 & 24.182 & Prob $>F$ \\
\hline Total & 24 & 1674.0028 & & $0.0007^{\star}$ \\
\hline
\end{tabular}

\section{Parameter Estimates}

\begin{tabular}{lrrrrrr} 
Term & Estimate & Std Error & t Ratio & Prob $>|\mathbf{t}|$ & Lower 95\% & Upper 95\% \\
\hline Intercept & 15.768143 & 1.85865 & 8.48 & $<.0001^{*}$ & 11.82798 & 19.708306 \\
\hline A & -0.311667 & 1.159072 & -0.27 & 0.7914 & -2.76879 & 2.1454567 \\
B & 1.3612778 & 1.159072 & 1.17 & 0.2574 & -1.095846 & 3.8184011 \\
C & 1.7221111 & 1.159072 & 1.49 & 0.1568 & -0.735012 & 4.1792344 \\
D & 0.7593333 & 1.159072 & 0.66 & 0.5217 & -1.69779 & 3.2164567 \\
$B^{\star} \mathrm{C}$ & 3.8800625 & 1.229382 & 3.16 & $0.0061^{*}$ & 1.2738896 & 6.4862354 \\
$B^{\star} \mathrm{D}$ & -0.376813 & 1.229382 & -0.31 & 0.7632 & -2.982985 & 2.2293604 \\
$\mathrm{C}^{\star} \mathrm{D}$ & -3.929938 & 1.229382 & -3.20 & $0.0056^{*}$ & -6.53611 & -1.323765 \\
$\mathrm{C}^{\star} \mathrm{C}$ & 11.767968 & 2.190441 & 5.37 & $<.0001^{*}$ & 7.1244416 & 16.411495
\end{tabular}




\section{Method 1 - Superior Region Stress:}

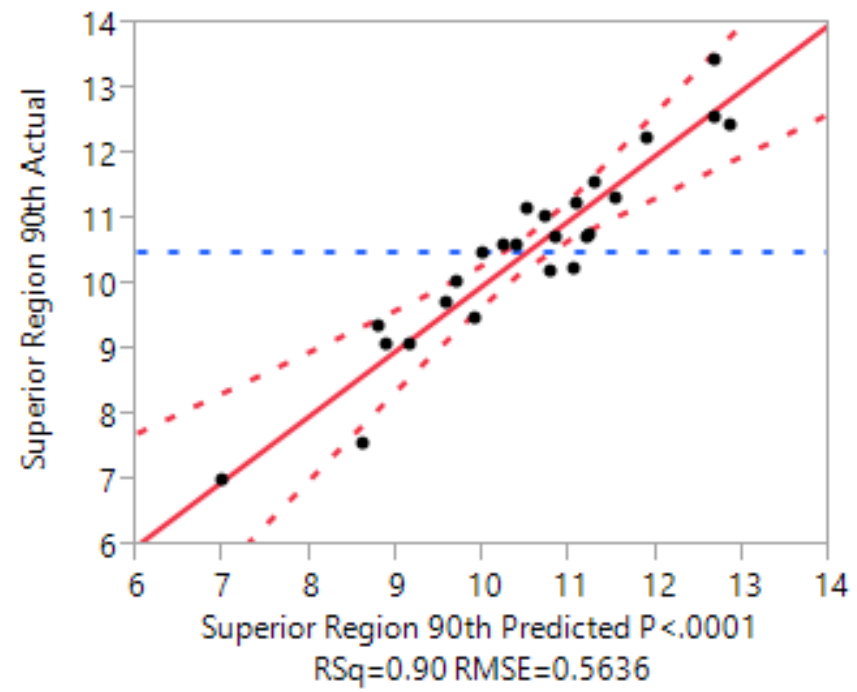

\section{Summary of Fit}

\begin{tabular}{l|r}
\hline RSquare & 0.900533 \\
RSquare Adj & 0.850799 \\
Root Mean Square Error & 0.563648 \\
Mean of Response & 10.50448 \\
Observations (or Sum Wgts) & 25
\end{tabular}

\section{Analysis of Variance}

\begin{tabular}{|c|c|c|c|c|}
\hline Source & DF & $\begin{array}{l}\text { Sum of } \\
\text { Squares }\end{array}$ & Mean Square & F Ratio \\
\hline Model & 8 & 46.020924 & 5.75262 & 18.1071 \\
\hline Error & 16 & 5.083182 & 0.31770 & Prob $>F$ \\
\hline C. Total & 24 & 51.104106 & & $<.0001^{*}$ \\
\hline
\end{tabular}

\section{Parameter Estimates}

\begin{tabular}{lrrrrrr}
\hline Term & Estimate & Std Error & t Ratio & Prob $>|\mathbf{t}|$ & Lower 95\% & Upper 95\% \\
Intercept & 10.730857 & 0.213039 & 50.37 & $<.0001^{*}$ & 10.279235 & 11.182479 \\
A & -0.8095 & 0.132853 & -6.09 & $<.0001^{*}$ & -1.091136 & -0.527864 \\
B & 0.4792778 & 0.132853 & 3.61 & $0.0024^{*}$ & 0.1976418 & 0.7609137 \\
C & -0.413222 & 0.132853 & -3.11 & $0.0067^{*}$ & -0.694858 & -0.131586 \\
D & -1.152 & 0.132853 & -8.67 & $<.0001^{*}$ & -1.433636 & -0.870364 \\
$B^{\star} \mathrm{C}$ & 0.380125 & 0.140912 & 2.70 & $0.0158^{*}$ & 0.081405 & 0.678845 \\
$B^{\star} D$ & 0.0905 & 0.140912 & 0.64 & 0.5298 & -0.20822 & 0.38922 \\
$C^{\star} D$ & -0.1085 & 0.140912 & -0.77 & 0.4525 & -0.40722 & 0.19022 \\
$C^{\star} \mathrm{C}$ & -0.314413 & 0.251069 & -1.25 & 0.2285 & -0.846655 & 0.2178292
\end{tabular}




\section{Method 1 - Lateral Region Stress:}

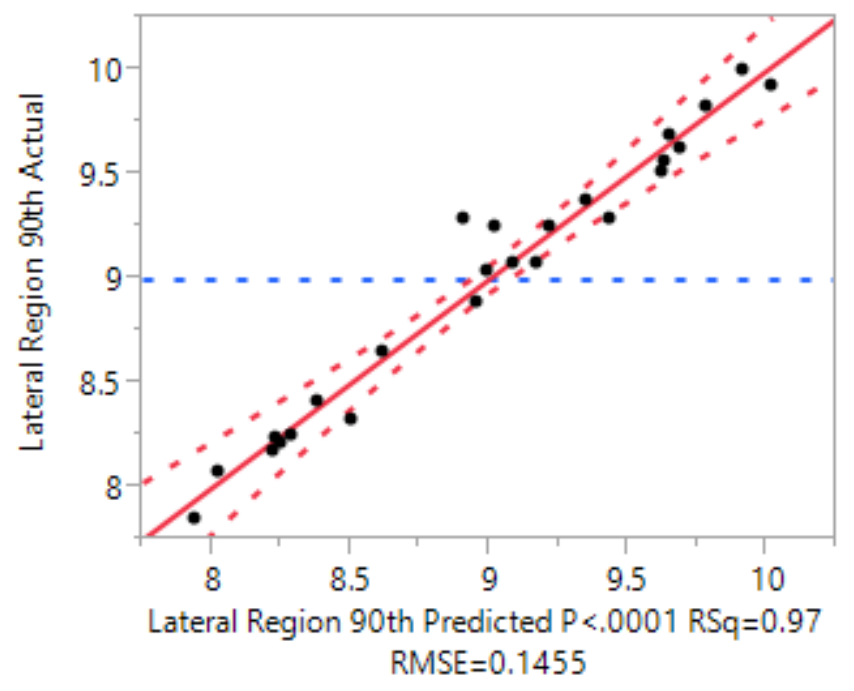

\begin{tabular}{|l|r|}
\hline Summary of Fit & \\
\hline RSquare & 0.966234 \\
RSquare Adj & 0.949351 \\
Root Mean Square Error & 0.14555 \\
Mean of Response & 8.994 \\
Observations (or Sum Wgts) & 25 \\
\hline
\end{tabular}

\begin{tabular}{|c|c|c|c|c|}
\hline \multicolumn{5}{|c|}{ Analysis of Variance } \\
\hline Source & DF & $\begin{array}{r}\text { Sum of } \\
\text { Squares }\end{array}$ & Mean Square & F Ratio \\
\hline Model & 8 & 9.699510 & 1.21244 & 57.2316 \\
\hline Error & 16 & 0.338956 & 0.02118 & Prob $>F$ \\
\hline C. Total & 24 & 10.038466 & & $<.0001^{*}$ \\
\hline
\end{tabular}

\begin{tabular}{|c|c|c|c|c|c|c|}
\hline \multicolumn{7}{|c|}{ Parameter Estimates } \\
\hline Term & Estimate & Std Error & t Ratio & Prob $>|t|$ & Lower $95 \%$ & Upper 95\% \\
\hline Intercept & 9.0821429 & 0.055013 & 165.09 & $<.0001^{*}$ & 8.9655211 & 9.1987646 \\
\hline A & -0.702278 & 0.034306 & -20.47 & $<.0001^{*}$ & -0.775004 & -0.629551 \\
\hline B & 0.0907222 & 0.034306 & 2.64 & $0.0177^{\star}$ & 0.0179958 & 0.1634486 \\
\hline C & 0.0573889 & 0.034306 & 1.67 & 0.1138 & -0.015338 & 0.1301153 \\
\hline D & 0.1328889 & 0.034306 & 3.87 & $0.0013^{*}$ & 0.0601625 & 0.2056153 \\
\hline $\mathrm{B}^{\star} \mathrm{C}$ & -0.0295 & 0.036387 & -0.81 & 0.4294 & -0.106638 & 0.047638 \\
\hline$B^{\star} \mathrm{D}$ & 0.077875 & 0.036387 & 2.14 & $0.0481^{*}$ & 0.000737 & 0.155013 \\
\hline$C^{\star} \mathrm{D}$ & -0.083 & 0.036387 & -2.28 & $0.0366^{*}$ & -0.160138 & -0.005862 \\
\hline $\mathrm{C}^{\star} \mathrm{C}$ & -0.122421 & 0.064833 & -1.89 & 0.0773 & -0.259861 & 0.0150194 \\
\hline
\end{tabular}




\section{Method 2 - Proximal Region \% of Intact:}

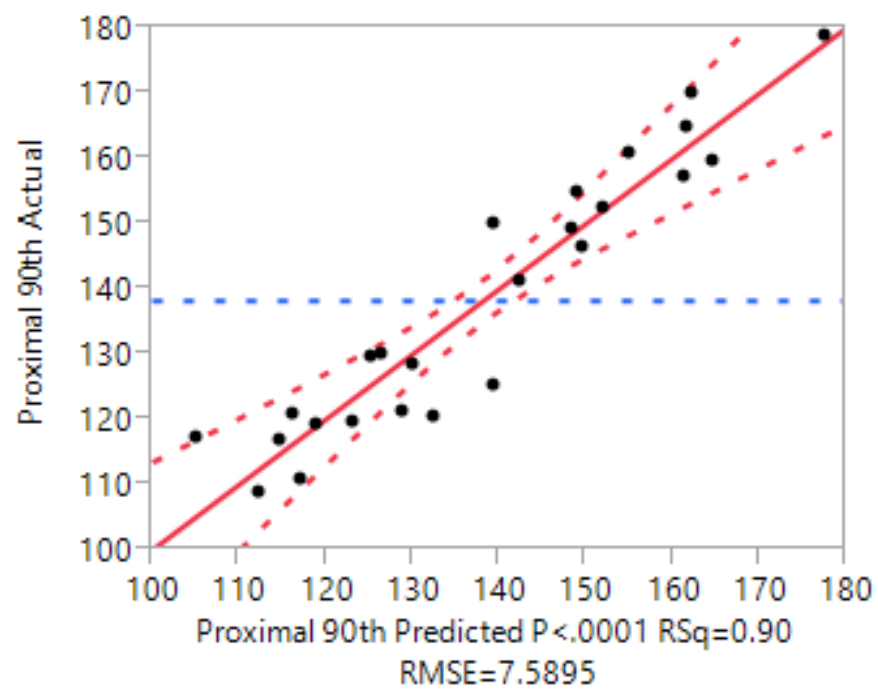

\begin{tabular}{l|r}
\hline Summary of Fit & \\
\hline RSquare & 0.902673 \\
RSquare Adj & 0.862597 \\
Root Mean Square Error & 7.589494 \\
Mean of Response & 138.1478 \\
Observations (or Sum Wgts) & 25
\end{tabular}

\section{Analysis of Variance}

\begin{tabular}{lrrrrr|} 
& & Sum of & & \\
Source & DF & Squares & Mean Square & F Ratio \\
\hline Model & 7 & 9081.798 & 1297.40 & 22.5241 \\
\hline Error & 17 & 979.207 & 57.60 & Prob > F \\
C. Total & 24 & 10061.005 & & $<.0001^{*}$
\end{tabular}

\section{Parameter Estimates}

$\begin{array}{lrrrrrr}\text { Term } & \text { Estimate } & \text { Std Error } & \text { t Ratio } & \text { Prob }>|\mathbf{t}| & \text { Lower 95\% } & \text { Upper 95\% } \\ \text { Intercept } & 118.84729 & 2.868559 & 41.43 & <.0001^{*} & 112.79516 & 124.89942 \\ \text { A } & -6.379056 & 1.788861 & -3.57 & 0.0024^{*} & -10.15322 & -2.604889 \\ \text { B } & 4.2104444 & 1.788861 & 2.35 & 0.0309^{*} & 0.4362781 & 7.9846108 \\ \text { C } & 6.3755 & 1.788861 & 3.56 & 0.0024^{*} & 2.6013336 & 10.149666 \\ \text { D } & 13.657333 & 1.788861 & 7.63 & <.0001^{*} & 9.8831669 & 17.4315 \\ B^{\star} \mathrm{C} & 3.711625 & 1.897373 & 1.96 & 0.0671 & -0.291483 & 7.714733 \\ \text { C }^{\star} \mathrm{D} & -2.489375 & 1.897373 & -1.31 & 0.2070 & -6.492483 & 1.513733 \\ \mathrm{C}^{\star} \mathrm{C} & 26.806214 & 3.380629 & 7.93 & <.0001^{*} & 19.67371 & 33.938718\end{array}$


Method 2 - Inferior Region \% of Intact:

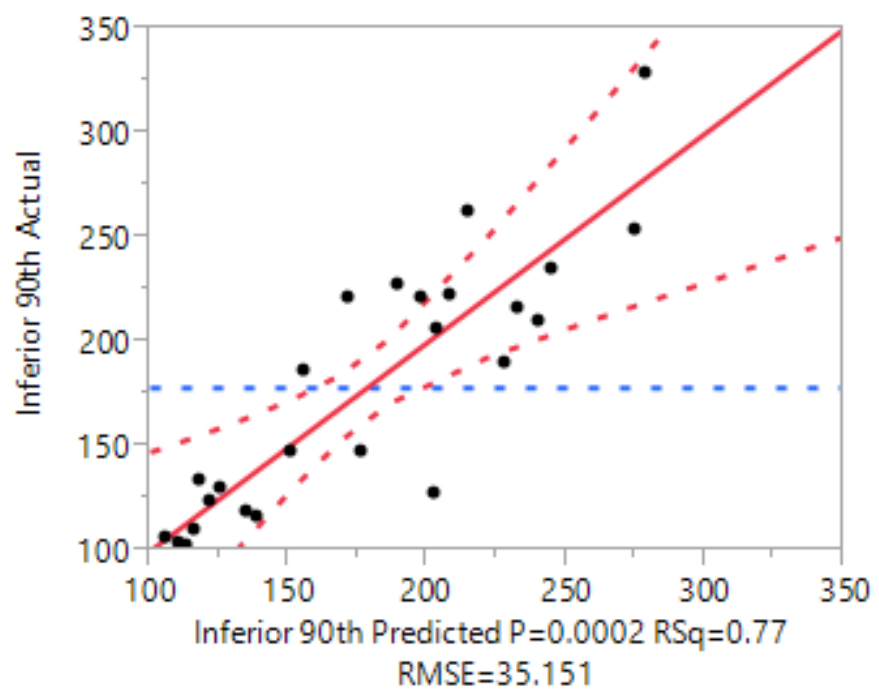

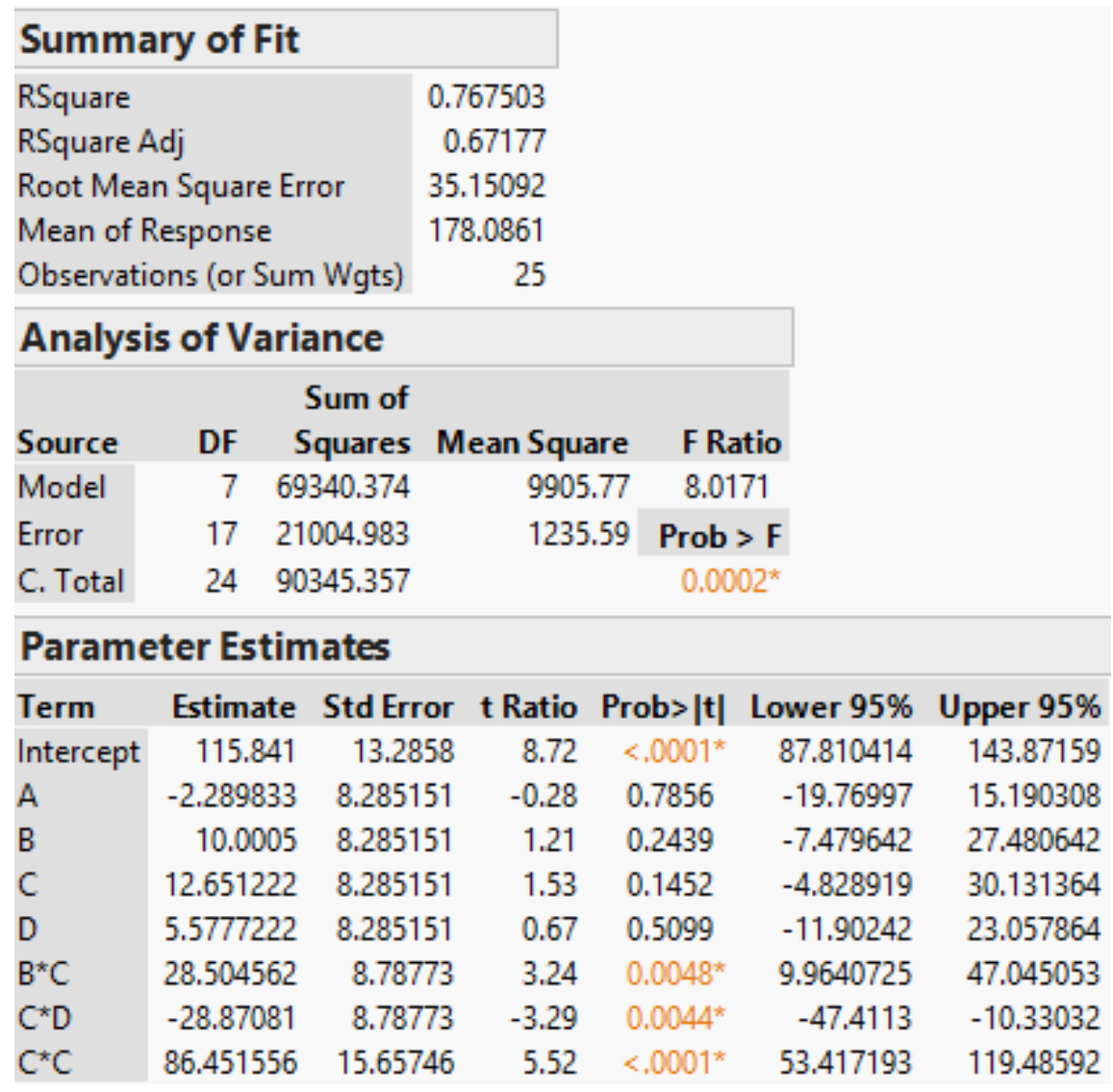




\section{Method 2 - Superior Region \% of Intact:}

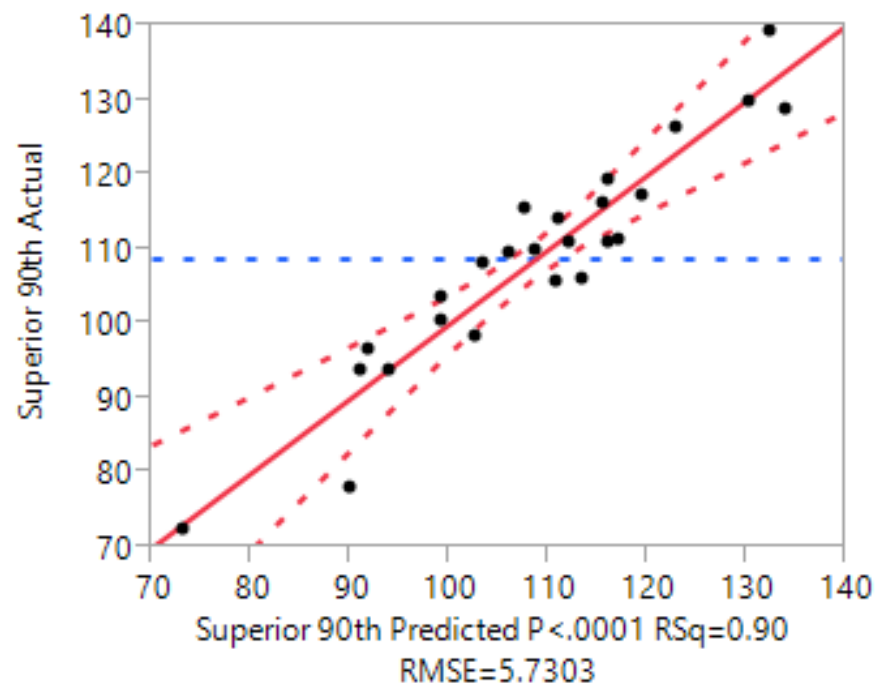

\begin{tabular}{|c|c|c|c|c|}
\hline \multicolumn{4}{|c|}{ Summary of Fit } & \\
\hline \multicolumn{3}{|c|}{ RSquare } & 0.898005 & \\
\hline \multicolumn{3}{|c|}{ RSquare Adj } & 0.856008 & \\
\hline \multicolumn{3}{|c|}{ Root Mean Square Error } & 5.730297 & \\
\hline \multicolumn{3}{|c|}{ Mean of Response } & 108.7023 & \\
\hline \multicolumn{3}{|c|}{ Observations (or Sum Wgts) } & 25 & \\
\hline \multicolumn{5}{|c|}{ Analysis of Variance } \\
\hline \multirow{2}{*}{$\begin{array}{l}\text { Source } \\
\text { Model }\end{array}$} & DF & $\begin{array}{r}\text { Sum of } \\
\text { Squares }\end{array}$ & Mean Square & F Ratio \\
\hline & 7 & 4914.7894 & 702.113 & 21.3822 \\
\hline Error & 17 & 558.2172 & 32.836 & Prob $>\mathrm{F}$ \\
\hline C. Total & 24 & 5473.0066 & & $<.0001^{*}$ \\
\hline
\end{tabular}

\section{Parameter Estimates}

\begin{tabular}{lrrrrrr}
\hline Term & Estimate & Std Error & t Ratio & Prob $>|\mathbf{t}|$ & Lower 95\% & Upper 95\% \\
Intercept & 111.04586 & 2.165849 & 51.27 & $<.0001^{*}$ & 106.47632 & 115.6154 \\
A & -8.377889 & 1.350644 & -6.20 & $<.0001^{*}$ & -11.2275 & -5.528279 \\
B & 4.9595 & 1.350644 & 3.67 & $0.0019^{*}$ & 2.1098902 & 7.8091098 \\
C & -4.275222 & 1.350644 & -3.17 & $0.0057^{\star}$ & -7.124832 & -1.425612 \\
D & -11.92183 & 1.350644 & -8.83 & $<.0001^{*}$ & -14.77144 & -9.072224 \\
$B^{\star} \mathrm{C}$ & 3.93475 & 1.432574 & 2.75 & $0.0138^{*}$ & 0.9122824 & 6.9572176 \\
$C^{\star} D$ & -1.1235 & 1.432574 & -0.78 & 0.4437 & -4.145968 & 1.8989676 \\
$C^{\star} \mathrm{C}$ & -3.254968 & 2.552477 & -1.28 & 0.2194 & -8.640225 & 2.130288
\end{tabular}




\section{Method 2 - Lateral Region \% of Intact:}

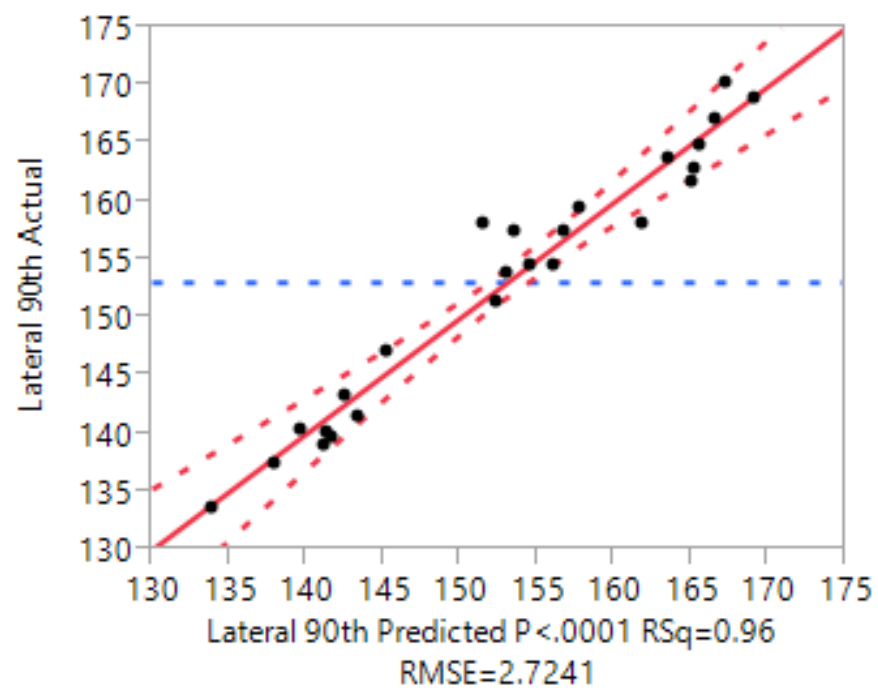

\section{Summary of Fit}

\begin{tabular}{l|r}
\hline RSquare & 0.956606 \\
RSquare Adj & 0.938738 \\
Root Mean Square Error & 2.724056 \\
Mean of Response & 153.0454 \\
Observations (or Sum Wgts) & 25
\end{tabular}

\section{Analysis of Variance}

Sum of

\begin{tabular}{lrrrrr} 
Source & DF & Squares & Mean Square & F Ratio \\
\hline Model & 7 & 2780.9021 & 397.272 & 53.5372 \\
\hline Error & 17 & 126.1482 & 7.420 & Prob $>$ F \\
C. Total & 24 & 2907.0503 & & $<.0001^{*}$
\end{tabular}

\section{Parameter Estimates}

\begin{tabular}{lrrrrrr} 
Term & Estimate & Std Error & t Ratio & Prob $>|\mathbf{t}|$ & Lower 95\% & Upper 95\% \\
\hline Intercept & 154.54314 & 1.029597 & 150.10 & $<.0001^{*}$ & 152.37088 & 156.7154 \\
A & -11.95094 & 0.642066 & -18.61 & $<.0001^{*}$ & -13.30559 & -10.5963 \\
B & 1.5449444 & 0.642066 & 2.41 & $0.0278^{*}$ & 0.190303 & 2.8995859 \\
C & 0.9774444 & 0.642066 & 1.52 & 0.1463 & -0.377197 & 2.3320859 \\
D & 2.2623889 & 0.642066 & 3.52 & $0.0026^{\star}$ & 0.9077475 & 3.6170303 \\
$B^{\star} \mathrm{C}$ & -0.502125 & 0.681014 & -0.74 & 0.4710 & -1.938939 & 0.9346892 \\
$C^{\star} \mathrm{D}$ & -1.412375 & 0.681014 & -2.07 & 0.0536 & -2.849189 & 0.0244392 \\
$\mathrm{C}^{\star} \mathrm{C}$ & -2.080143 & 1.213391 & -1.71 & 0.1046 & -4.640175 & 0.4798888
\end{tabular}




\section{Appendix D: Complete Extension Phase DOE Results Approach 1 Outcomes - Proximal Region}

\section{Method 1 - Mean Stress Model:}

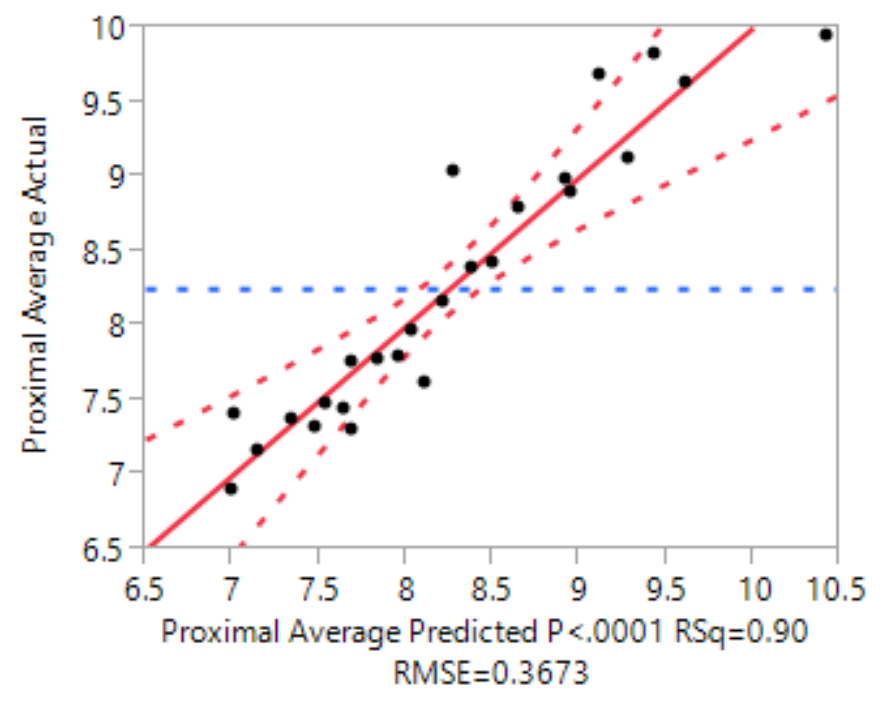

\begin{tabular}{|c|c|c|c|c|c|c|}
\hline \multicolumn{4}{|c|}{ Summary of Fit } & & & \\
\hline \multicolumn{3}{|l|}{ RSquare } & \multicolumn{4}{|l|}{0.901703} \\
\hline \multicolumn{3}{|c|}{ RSquare Adj } & \multicolumn{4}{|l|}{0.842724} \\
\hline \multicolumn{3}{|c|}{ Root Mean Square Error } & \multicolumn{4}{|l|}{0.367287} \\
\hline \multicolumn{3}{|c|}{ Mean of Response } & \multicolumn{4}{|l|}{8.24896} \\
\hline \multicolumn{3}{|c|}{ Observations (or Sum Wgts) } & 25 & & & \\
\hline \multicolumn{7}{|c|}{ Analysis of Variance } \\
\hline \multicolumn{5}{|c|}{ Sum of } & F Ratio & \\
\hline Model & 9 & 18.561992 & 2.06244 & \multicolumn{2}{|c|}{15.2887} & \\
\hline Error & 15 & 2.023497 & 0.1349 & \multicolumn{2}{|c|}{ Prob $>F$} & \\
\hline C. Total & $24 \quad 20$ & 0.585489 & & $<.00$ & $201^{*}$ & \\
\hline \multicolumn{7}{|c|}{ Parameter Estimates } \\
\hline Term & Estimate & Std Error & or $t$ Ratio & Prob $>|t|$ & Lower $95 \%$ & Upper $95 \%$ \\
\hline Intercept & 7.343 & 0.138821 & $21 \quad 52.90$ & $<.0001^{*}$ & 7.0471091 & 7.6388909 \\
\hline A & -0.345889 & 0.08657 & -4.00 & $0.0012^{*}$ & -0.530409 & -0.161368 \\
\hline B & 0.1925 & 0.08657 & 2.22 & $0.0420^{*}$ & 0.0079796 & 0.3770204 \\
\hline C & 0.3245 & 0.08657 & 3.75 & $0.0019^{*}$ & 0.1399796 & 0.5090204 \\
\hline D & 0.3377778 & 0.08657 & 3.90 & $0.0014^{*}$ & 0.1532574 & 0.5222982 \\
\hline$A^{*} B$ & -0.217937 & 0.091822 & -2.37 & $0.0314^{*}$ & -0.413651 & -0.022224 \\
\hline $\mathrm{B}^{\star} \mathrm{C}$ & 0.2461875 & 0.091822 & 2.68 & $0.0171^{*}$ & 0.050474 & 0.441901 \\
\hline$A^{*} D$ & -0.323313 & 0.091822 & -3.52 & $0.0031^{*}$ & -0.519026 & -0.127599 \\
\hline$C^{\star} \mathrm{D}$ & -0.160188 & 0.091822 & -1.74 & 0.1015 & -0.355901 & 0.035526 \\
\hline $\mathrm{C}^{\star} \mathrm{C}$ & 1.2582778 & 0.163603 & 7.69 & $<.0001^{*}$ & 0.909567 & 1.6069886 \\
\hline
\end{tabular}




\section{Method 1 - Stress Standard Deviation Model:}

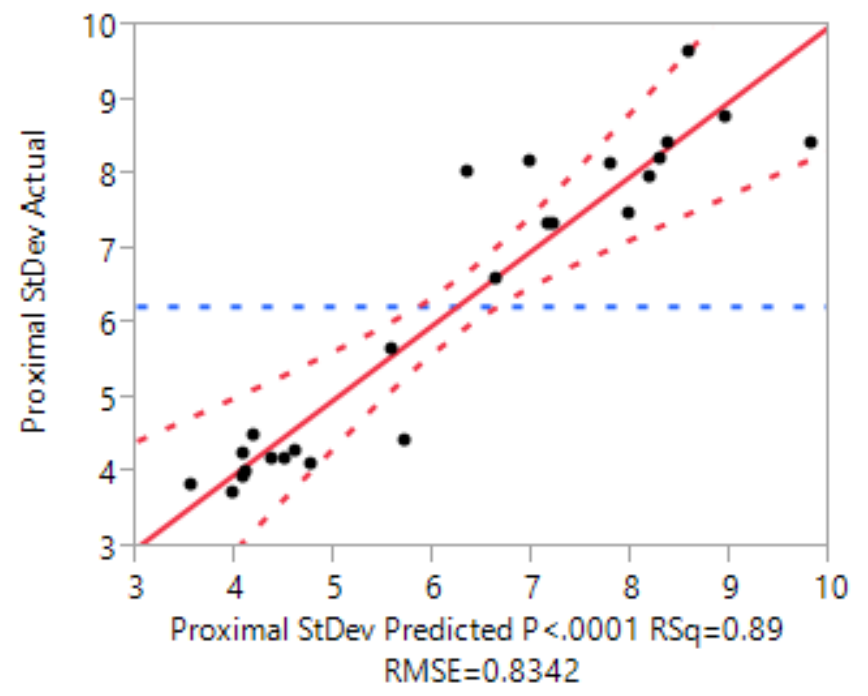

\section{Summary of Fit}

\begin{tabular}{l|r}
\hline RSquare & 0.894579 \\
RSquare Adj & 0.831327 \\
Root Mean Square Error & 0.834157 \\
Mean of Response & 6.23052 \\
Observations (or Sum Wgts) & 25
\end{tabular}

\begin{tabular}{|c|c|c|c|c|}
\hline \multicolumn{5}{|c|}{ Analysis of Variance } \\
\hline Source & DF & $\begin{array}{r}\text { Sum of } \\
\text { Squares }\end{array}$ & Mean Square & F Ratio \\
\hline Model & 9 & 88.568361 & 9.84093 & 14.1430 \\
\hline Error & 15 & 10.437263 & 0.69582 & Prob $>F$ \\
\hline C. Total & 24 & 99.005624 & & $<, 0001^{*}$ \\
\hline
\end{tabular}

\section{Parameter Estimates}

$\begin{array}{lrrrrrr}\text { Term } & \text { Estimate } & \text { Std Error } & \text { t Ratio } & \text { Prob }>\text { |t| } & \text { Lower 95\% } & \text { Upper 95\% } \\ \text { Intercept } & 4.0817143 & 0.315282 & 12.95 & <.0001^{*} & 3.4097074 & 4.7537212 \\ \text { A } & 0.0113333 & 0.196613 & 0.06 & 0.9548 & -0.407737 & 0.4304033 \\ \text { B } & 0.1127778 & 0.196613 & 0.57 & 0.5747 & -0.306292 & 0.5318477 \\ \text { C } & 0.7141667 & 0.196613 & 3.63 & 0.0025^{\star} & 0.2950967 & 1.1332366 \\ \text { D } & 0.5193333 & 0.196613 & 2.64 & 0.0185^{\star} & 0.1002634 & 0.9384033 \\ A^{\star} \mathrm{B} & -0.57125 & 0.208539 & -2.74 & 0.0152^{\star} & -1.015741 & -0.126759 \\ B^{\star} \mathrm{C} & 0.738625 & 0.208539 & 3.54 & 0.0030^{*} & 0.2941342 & 1.1831158 \\ \mathrm{~A}^{\star} \mathrm{D} & -0.746 & 0.208539 & -3.58 & 0.0028^{\star} & -1.190491 & -0.301509 \\ \mathrm{C}^{\star} \mathrm{D} & -0.640125 & 0.208539 & -3.07 & 0.0078^{\star} & -1.084616 & -0.195634 \\ \mathrm{C}^{\star} \mathrm{C} & 2.9844524 & 0.371563 & 8.03 & <.0001^{*} & 2.1924847 & 3.7764201\end{array}$




\section{Method $1-90^{\text {th }}$ Percentile Stress Model:}

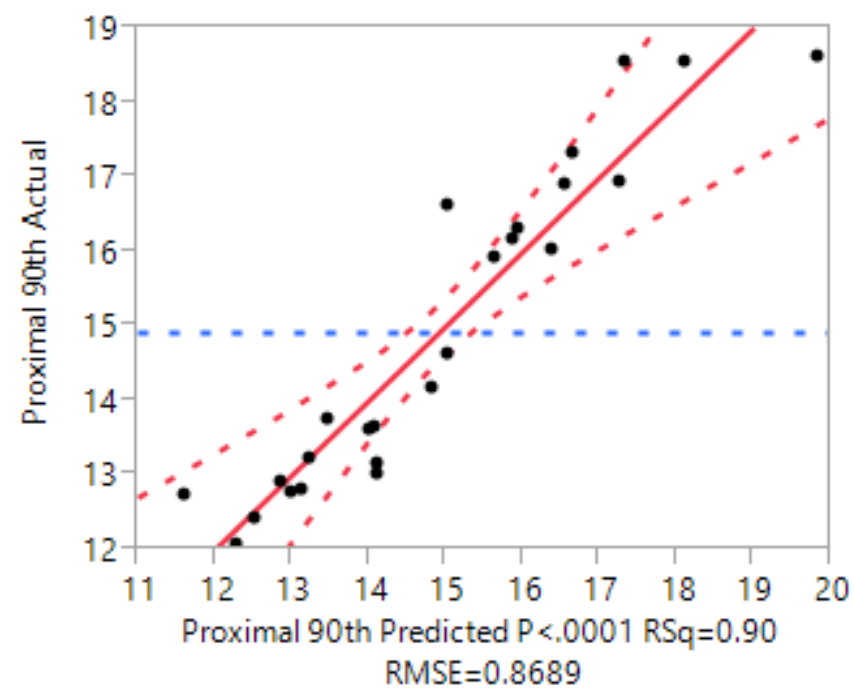

\section{Summary of Fit}

\begin{tabular}{lr}
\hline RSquare & 0.896626 \\
RSquare Adj & 0.834602 \\
Root Mean Square Error & 0.86893 \\
Mean of Response & 14.91264 \\
Observations (or Sum Wgts) & 25 \\
\hline
\end{tabular}

\section{Analysis of Variance}

\begin{tabular}{lrrrrr} 
& & Sum of & & \\
Source & DF & Squares & Mean Square & F Ratio \\
\hline Model & 9 & 98.23377 & 10.9149 & 14.4560 \\
Error & 15 & 11.32558 & 0.7550 & Prob $>$ F \\
C. Total & 24 & 109.55935 & & $<.0001^{*}$
\end{tabular}

\section{Parameter Estimates}

$\begin{array}{lrrrrrr}\text { Term } & \text { Estimate } & \text { Std Error } & \mathbf{t} \text { Ratio } & \text { Prob }>|\mathbf{t}| & \text { Lower 95\% } & \text { Upper 95\% } \\ \text { Intercept } & 12.87 & 0.328425 & 39.19 & <.0001^{*} & 12.16998 & 13.57002 \\ \mathrm{~A} & -0.594222 & 0.204809 & -2.90 & 0.0110^{*} & -1.030762 & -0.157683 \\ \mathrm{~B} & 0.3618333 & 0.204809 & 1.77 & 0.0976 & -0.074706 & 0.7983727 \\ \mathrm{C} & 0.6735556 & 0.204809 & 3.29 & 0.0050^{*} & 0.2370162 & 1.1100949 \\ \mathrm{D} & 1.2533333 & 0.204809 & 6.12 & <.0001^{*} & 0.816794 & 1.6898727 \\ \mathrm{~A}^{\star} \mathrm{B} & -0.483 & 0.217232 & -2.22 & 0.0420^{*} & -0.94602 & -0.01998 \\ \mathrm{~B}^{\star} \mathrm{C} & 0.413375 & 0.217232 & 1.90 & 0.0764 & -0.049645 & 0.8763949 \\ \mathrm{~A}^{\star} \mathrm{D} & -0.57175 & 0.217232 & -2.63 & 0.0189^{*} & -1.03477 & -0.10873 \\ \mathrm{C}^{\star} \mathrm{D} & -0.226125 & 0.217232 & -1.04 & 0.3144 & -0.689145 & 0.2368949 \\ \mathrm{C}^{\star} \mathrm{C} & 2.837 & 0.387052 & 7.33 & <.0001^{*} & 2.0120182 & 3.6619818\end{array}$




\section{Proximal Region Stress Desirability:}

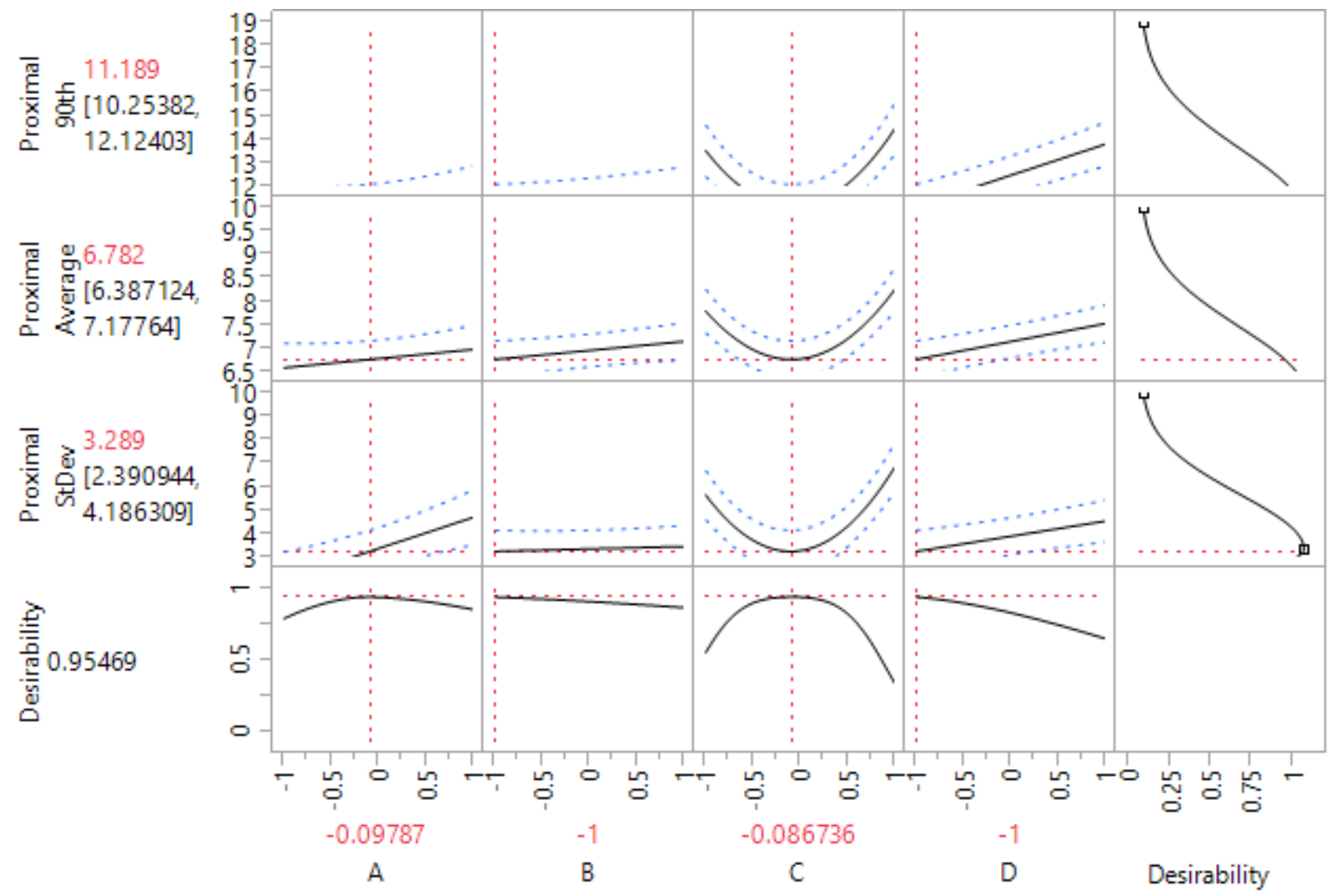


Method 2 - Mean \% of Intact Stress Model:

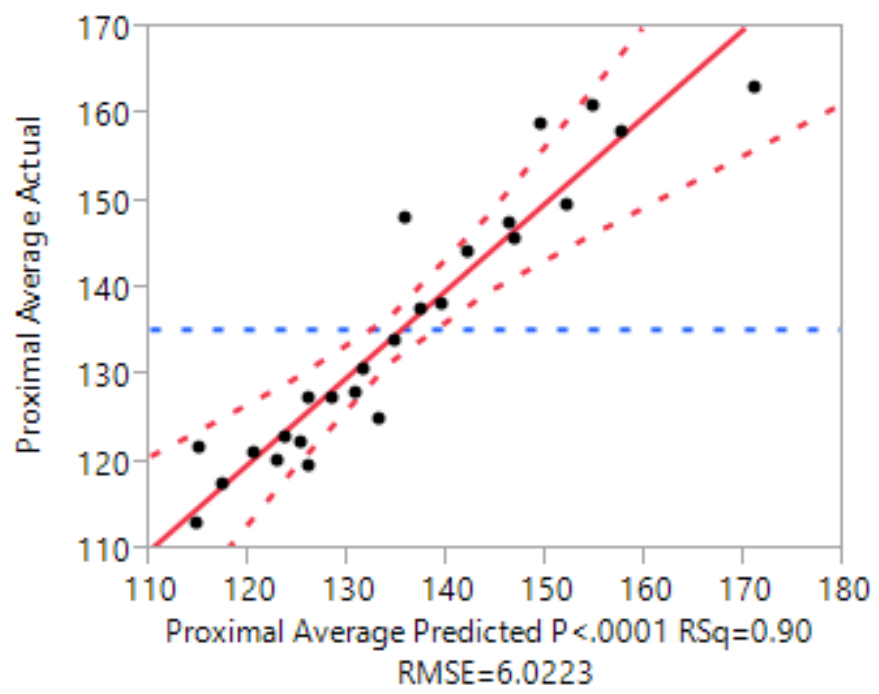

\section{Summary of Fit}

\begin{tabular}{lr}
\hline RSquare & 0.901786 \\
RSquare Adj & 0.842857 \\
Root Mean Square Error & 6.022254 \\
Mean of Response & 135.3211 \\
Observations (or Sum Wgts) & 25
\end{tabular}

\section{Analysis of Variance}

\begin{tabular}{lrrrrr} 
& & \multicolumn{2}{c}{ Sum of } & & \\
Source & DF & Squares & Mean Square & F Ratio \\
Model & 9 & 4995.0235 & 555.003 & 15.3030 \\
Error & 15 & 544.0131 & 36.268 & Prob > F \\
C. Total & 24 & 5539.0366 & & $<.0001^{*}$
\end{tabular}

\section{Parameter Estimates}

$\begin{array}{lrrrrrr}\text { Term } & \text { Estimate } & \text { Std Error } & \text { t Ratio } & \text { Prob }>|\mathbf{t}| & \text { Lower 95\% } & \text { Upper 95\% } \\ \text { Intercept } & 120.46051 & 2.276198 & 52.92 & <.0001^{*} & 115.60891 & 125.31212 \\ \text { A } & -5.675418 & 1.419459 & -4.00 & 0.0012^{\star} & -8.700923 & -2.649913 \\ \text { B } & 3.1578305 & 1.419459 & 2.22 & 0.0419^{\star} & 0.1323256 & 6.1833353 \\ \text { C } & 5.3220977 & 1.419459 & 3.75 & 0.0019^{\star} & 2.2965928 & 8.3476025 \\ \text { D } & 5.5409396 & 1.419459 & 3.90 & 0.0014^{\star} & 2.5154348 & 8.5664445 \\ \text { A }^{\star} \mathrm{B} & -3.574648 & 1.505563 & -2.37 & 0.0314^{\star} & -6.78368 & -0.365615 \\ \mathrm{~B}^{\star} \mathrm{C} & 4.0375332 & 1.505563 & 2.68 & 0.0171^{\star} & 0.8285007 & 7.2465657 \\ \mathrm{~A}^{\star} \mathrm{D} & -5.306571 & 1.505563 & -3.52 & 0.0031^{\star} & -8.515603 & -2.097538 \\ \mathrm{C}^{\star} \mathrm{D} & -2.626808 & 1.505563 & -1.74 & 0.1015 & -5.83584 & 0.5822246 \\ \mathrm{C}^{\star} \mathrm{C} & 20.63971 & 2.682525 & 7.69 & <.0001^{\star} & 14.922043 & 26.357376\end{array}$




\section{Method 2 - \% of Intact Stress Standard Deviation Model:}

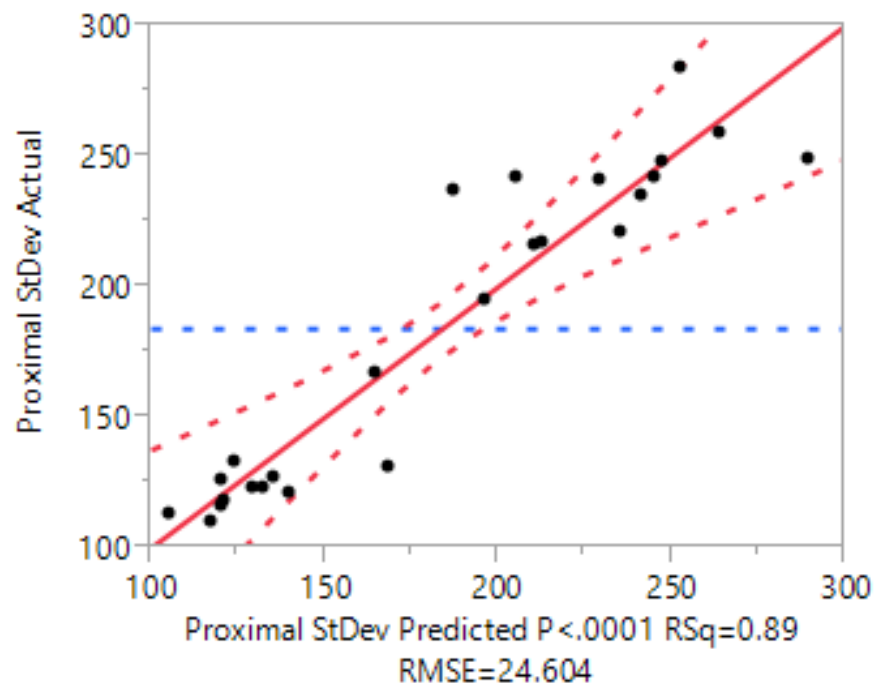

\begin{tabular}{|c|c|c|c|c|}
\hline \multicolumn{4}{|c|}{ Summary of Fit } & \\
\hline \multicolumn{3}{|l|}{ RSquare } & 0.894546 & \\
\hline \multicolumn{3}{|c|}{ RSquare Adj } & 0.831273 & \\
\hline \multicolumn{3}{|c|}{ Root Mean Square Error } & 24.60414 & \\
\hline \multicolumn{3}{|c|}{ Mean of Response } & 183.7604 & \\
\hline \multicolumn{3}{|c|}{ Observations (or Sum Wgts) } & 25 & \\
\hline \multicolumn{5}{|c|}{ Analysis of Variance } \\
\hline Source & DF & $\begin{array}{l}\text { Sum of } \\
\text { Squares }\end{array}$ & Mean Square & F Ratio \\
\hline Model & 9 & 77027.672 & 8558.63 & 14.1380 \\
\hline Error & 15 & 9080.454 & 605.36 & Prob $>F$ \\
\hline C. Total & 24 & 86108.126 & & $<.0001^{*}$ \\
\hline
\end{tabular}

\section{Parameter Estimates}

$\begin{array}{lrrrrrr}\text { Term } & \text { Estimate } & \text { Std Error } & \text { t Ratio } & \text { Prob }>|\mathbf{t}| & \text { Lower 95\% } & \text { Upper 95\% } \\ \text { Intercept } & 120.39211 & 9.29949 & 12.95 & <.0001^{*} & 100.57071 & 140.2135 \\ \text { A } & 0.3352111 & 5.799251 & 0.06 & 0.9547 & -12.0256 & 12.696022 \\ \text { B } & 3.3237075 & 5.799251 & 0.57 & 0.5751 & -9.037103 & 15.684518 \\ \text { C } & 21.063228 & 5.799251 & 3.63 & 0.0025^{*} & 8.7024174 & 33.424039 \\ \text { D } & 15.312249 & 5.799251 & 2.64 & 0.0185^{*} & 2.9514387 & 27.67306 \\ \text { A }^{*} \mathrm{~B} & -16.8452 & 6.151034 & -2.74 & 0.0152^{*} & -29.95582 & -3.734584 \\ \text { B }^{\star} \mathrm{C} & 21.78173 & 6.151034 & 3.54 & 0.0030^{*} & 8.6711103 & 34.89235 \\ \text { A }^{*} \mathrm{D} & -22.00447 & 6.151034 & -3.58 & 0.0028^{*} & -35.11509 & -8.893853 \\ \mathrm{C}^{\star} \mathrm{D} & -18.87825 & 6.151034 & -3.07 & 0.0078^{*} & -31.98887 & -5.767628 \\ \mathrm{C}^{*} \mathrm{C} & 88.01146 & 10.95955 & 8.03 & <.0001^{*} & 64.651724 & 111.3712\end{array}$




\section{Method $2-90^{\text {th }}$ Percentile \% of Intact Stress Model:}

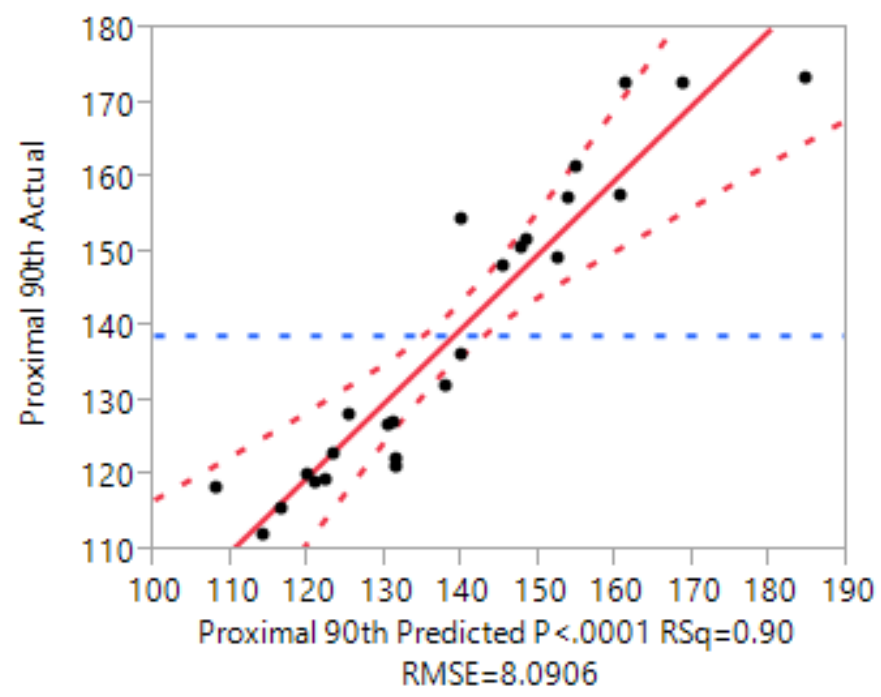

\section{Summary of Fit}

\begin{tabular}{lr}
\hline RSquare & 0.896623 \\
RSquare Adj & 0.834597 \\
Root Mean Square Error & 8.090644 \\
Mean of Response & 138.8471 \\
Observations (or Sum Wgts) & 25
\end{tabular}

Analysis of Variance

\begin{tabular}{|c|c|c|c|c|}
\hline Source & DF & $\begin{array}{l}\text { Sum of } \\
\text { Squares }\end{array}$ & Mean Square & F Ratio \\
\hline Model & 9 & 8516.1715 & 946.241 & 14.4556 \\
\hline Error & 15 & 981.8777 & 65.459 & Prob $>F$ \\
\hline C. Total & 24 & 9498.0492 & & $<.0001^{*}$ \\
\hline
\end{tabular}

\section{Parameter Estimates}

\begin{tabular}{lrrrrrr}
\hline Term & Estimate & Std Error & $\mathbf{t}$ Ratio & Prob $>|\mathbf{t}|$ & Lower 95\% & Upper 95\% \\
Intercept & 119.82892 & 3.057976 & 39.19 & $<.0001^{*}$ & 113.311 & 126.34684 \\
$\mathrm{~A}$ & -5.532796 & 1.906983 & -2.90 & $0.0110^{*}$ & -9.597434 & -1.468158 \\
$\mathrm{~B}$ & 3.3690348 & 1.906983 & 1.77 & 0.0976 & -0.695603 & 7.4336728 \\
$\mathrm{C}$ & 6.2721712 & 1.906983 & 3.29 & $0.0050^{*}$ & 2.2075332 & 10.336809 \\
$\mathrm{D}$ & 11.669444 & 1.906983 & 6.12 & $<.0001^{*}$ & 7.6048064 & 15.734082 \\
$\mathrm{~A}^{\star} \mathrm{B}$ & -4.497139 & 2.022661 & -2.22 & $0.0420^{*}$ & -8.808339 & -0.18594 \\
$\mathrm{~B}^{\star} \mathrm{C}$ & 3.8488799 & 2.022661 & 1.90 & 0.0764 & -0.46232 & 8.1600795 \\
$\mathrm{~A}^{\star} \mathrm{D}$ & -5.324281 & 2.022661 & -2.63 & $0.0188^{*}$ & -9.635481 & -1.013081 \\
$\mathrm{C}^{\star} \mathrm{D}$ & -2.105446 & 2.022661 & -1.04 & 0.3144 & -6.416646 & 2.2057533 \\
$\mathrm{C}^{\star} \mathrm{C}$ & 26.4142 & 3.603859 & 7.33 & $<.0001^{*}$ & 18.732756 & 34.095643
\end{tabular}




\section{Proximal Region \% of Intact Desirability:}

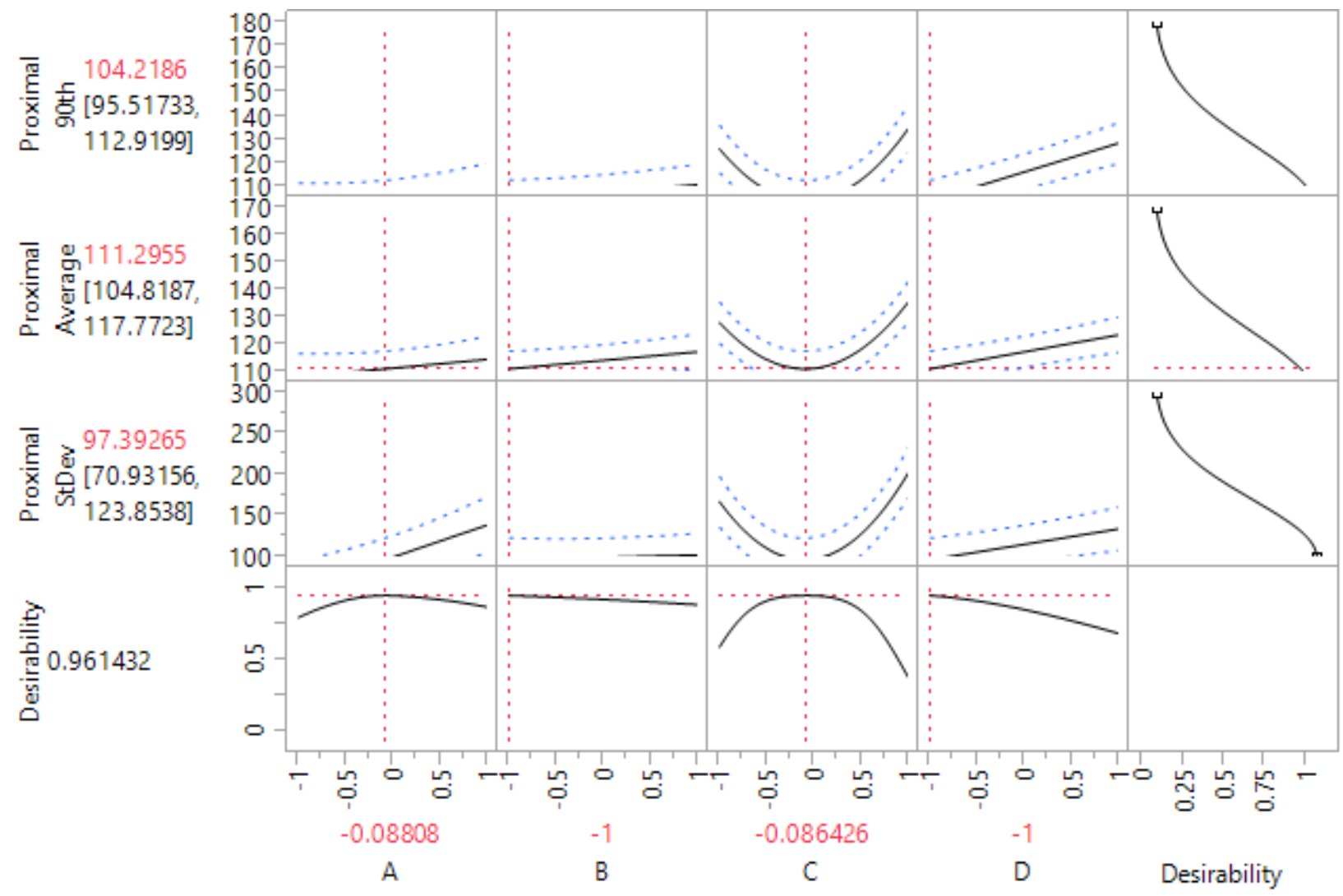




\section{Approach 2 Outcomes - All Regions Mean Stress}

\section{Method 1 - Proximal Region Stress:}

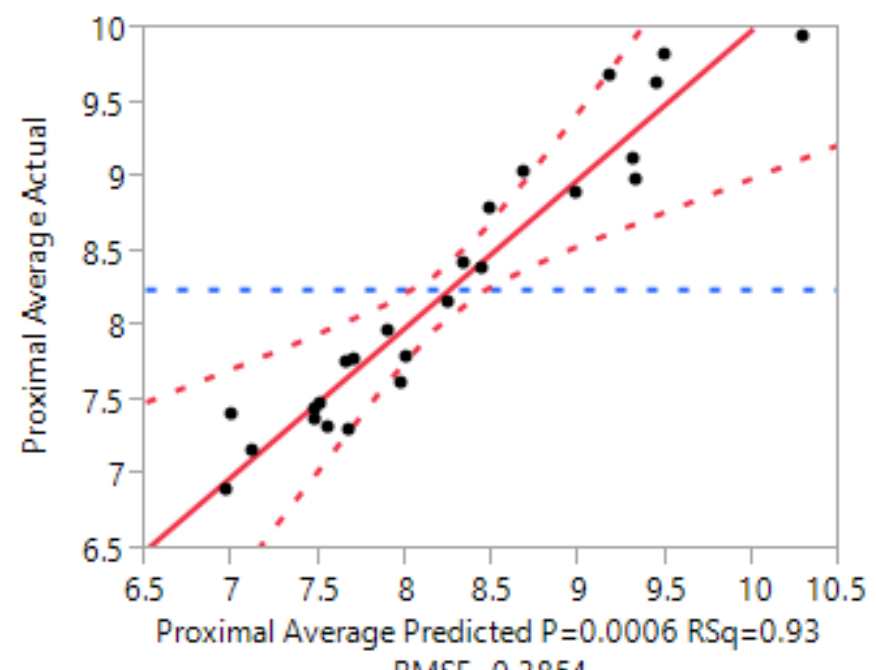

RMSE $=0.3854$

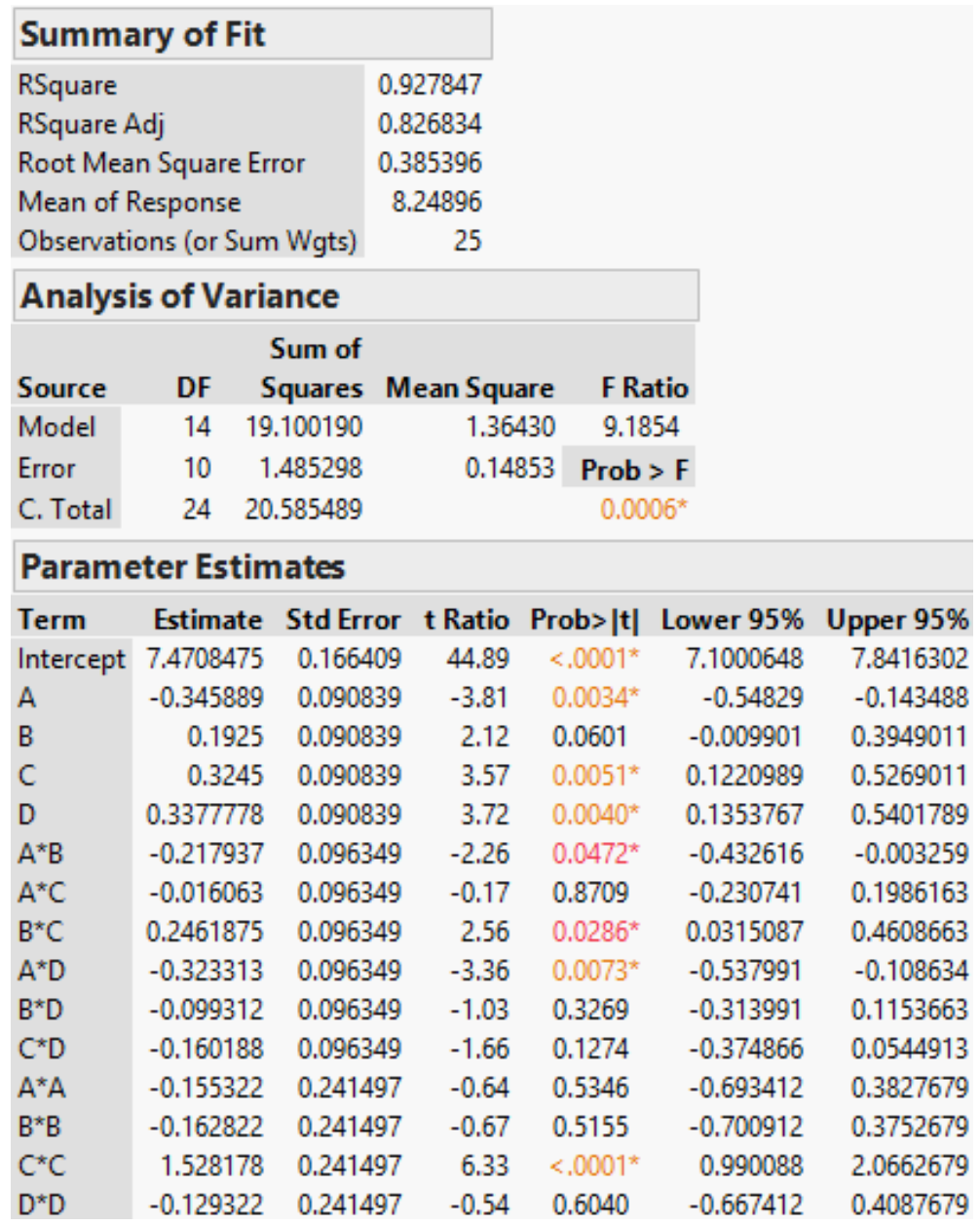




\section{Method 1 - Inferior Region Stress:}

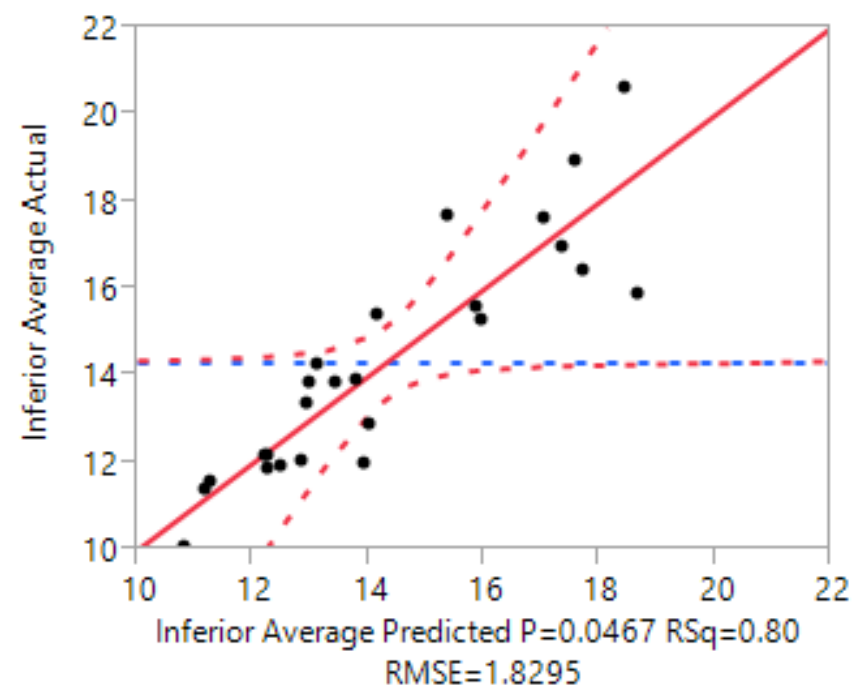

\begin{tabular}{lr|}
\hline Summary of Fit & \\
\hline RSquare & 0.803916 \\
RSquare Adj & 0.5294 \\
Root Mean Square Error & 1.829536 \\
Mean of Response & 14.3012 \\
Observations (or Sum Wgts) & 25 \\
\hline
\end{tabular}

Analysis of Variance

\begin{tabular}{|c|c|c|c|c|}
\hline Source & DF & $\begin{array}{l}\text { Sum of } \\
\text { Squares }\end{array}$ & Mean Square & F Ratio \\
\hline Model & 14 & 137.23092 & 9.80221 & 2.9285 \\
\hline Error & 10 & 33.47203 & 3.34720 & Prob $>F$ \\
\hline C. Total & 24 & 170.70295 & & $0.0467^{\star}$ \\
\hline
\end{tabular}

\section{Parameter Estimates}

\begin{tabular}{lrrrrrr} 
Term & Estimate & Std Error & t Ratio & Prob $>|\mathbf{t}|$ & Lower 95\% & Upper 95\% \\
\hline Intercept & 12.500661 & 0.789971 & 15.82 & $<.0001^{*}$ & 10.740495 & 14.260827 \\
$\mathrm{~A}$ & -1.075722 & 0.431226 & -2.49 & $0.0317^{\star}$ & -2.036553 & -0.114891 \\
$\mathrm{~B}$ & 0.4887222 & 0.431226 & 1.13 & 0.2835 & -0.472109 & 1.4495533 \\
$\mathrm{C}$ & 0.1700556 & 0.431226 & 0.39 & 0.7016 & -0.790776 & 1.1308866 \\
$\mathrm{D}$ & 0.5104444 & 0.431226 & 1.18 & 0.2639 & -0.450387 & 1.4712755 \\
$\mathrm{~A}^{\star} \mathrm{B}$ & -0.60025 & 0.457384 & -1.31 & 0.2187 & -1.619365 & 0.4188653 \\
$\mathrm{~A}^{\star} \mathrm{C}$ & -0.345125 & 0.457384 & -0.75 & 0.4679 & -1.36424 & 0.6739903 \\
$\mathrm{~B}^{\star} \mathrm{C}$ & 0.855375 & 0.457384 & 1.87 & 0.0910 & -0.16374 & 1.8744903 \\
$\mathrm{~A}^{\star} \mathrm{D}$ & -0.742875 & 0.457384 & -1.62 & 0.1354 & -1.76199 & 0.2762403 \\
$\mathrm{~B}^{\star} \mathrm{D}$ & -0.299625 & 0.457384 & -0.66 & 0.5272 & -1.31874 & 0.7194903 \\
$\mathrm{C}^{\star} \mathrm{D}$ & -0.8315 & 0.457384 & -1.82 & 0.0991 & -1.850615 & 0.1876153 \\
$\mathrm{~A}^{\star} \mathrm{A}$ & -0.621438 & 1.146428 & -0.54 & 0.5996 & -3.175839 & 1.9329631 \\
$\mathrm{~B}^{\star} \mathrm{B}$ & -0.745438 & 1.146428 & -0.65 & 0.5302 & -3.299839 & 1.8089631 \\
$\mathrm{C}^{\star} \mathrm{C}$ & 4.6875621 & 1.146428 & 4.09 & $0.0022^{*}$ & 2.1331612 & 7.2419631 \\
$\mathrm{D}^{\star} \mathrm{D}$ & -0.819938 & 1.146428 & -0.72 & 0.4908 & -3.374339 & 1.7344631
\end{tabular}




\section{Method 1 - Superior Region Stress:}

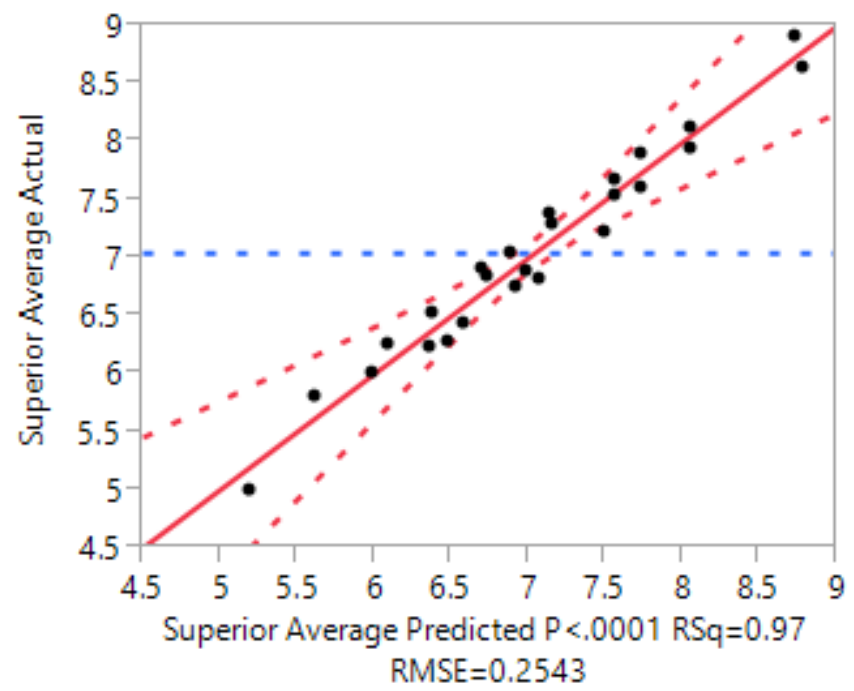

\section{Summary of Fit}

\begin{tabular}{lr}
\hline RSquare & 0.966723 \\
RSquare Adj & 0.920136 \\
\hline Root Mean Square Error & 0.254323 \\
Mean of Response & 7.03968 \\
\hline Observations (or Sum Wgts) & 25
\end{tabular}

Analysis of Variance

\begin{tabular}{|c|c|c|c|c|}
\hline Source & DF & $\begin{array}{l}\text { Sum of } \\
\text { Squares }\end{array}$ & Mean Square & F Ratio \\
\hline Model & 14 & 18.790422 & 1.34217 & 20.7509 \\
\hline Error & 10 & 0.646804 & 0.06468 & Prob $>F$ \\
\hline C. Total & 24 & 19.437225 & & $<.0001^{*}$ \\
\hline
\end{tabular}

\section{Parameter Estimates}

\begin{tabular}{|c|c|c|c|c|c|c|}
\hline Term & Estimate & Std Error & t Ratio & Prob $>|t|$ & Lower $95 \%$ & Upper $95 \%$ \\
\hline Intercept & 7.1399492 & 0.109814 & 65.02 & $<.0001^{*}$ & 6.8952689 & 7.3846294 \\
\hline A & -0.545889 & 0.059945 & -9.11 & $<.0001^{*}$ & -0.679454 & -0.412324 \\
\hline B & 0.3994444 & 0.059945 & 6.66 & $<.0001^{*}$ & 0.2658796 & 0.5330093 \\
\hline c & -0.094889 & 0.059945 & -1.58 & 0.1445 & -0.228454 & 0.038676 \\
\hline D & -0.733278 & 0.059945 & -12.23 & $<.0001^{*}$ & -0.866843 & -0.599713 \\
\hline$A^{\star} B$ & 0.0315 & 0.063581 & 0.50 & 0.6310 & -0.110167 & 0.1731669 \\
\hline$A^{*} \mathrm{C}$ & -0.01375 & 0.063581 & -0.22 & 0.8331 & & 0.1279169 \\
\hline $\mathrm{B}^{\star} \mathrm{C}$ & 0.133 & 0.063581 & 2.09 & 0.0629 & 8667 & 46669 \\
\hline$A^{\star} D$ & 0.001375 & 0.063581 & 0.02 & 0.9832 & 292 & 30419 \\
\hline$B^{\star} D$ & -0.109375 & 0.063581 & -1.72 & 0.1161 & -0.251042 & 0.0322919 \\
\hline$C^{\star} \mathrm{D}$ & 0.027625 & 0.063581 & 0.43 & 0.6732 & -0.114042 & 0.1692919 \\
\hline$A^{\star} A$ & -0.116441 & 0.159365 & -0.73 & 0.4818 & -0.471527 & 0.2386459 \\
\hline$B^{\star} B$ & -0.040441 & 0.159365 & -0.25 & 0.8048 & -0.395527 & 0.3146459 \\
\hline $\mathrm{C}^{\star} \mathrm{C}$ & -0.163441 & 0.159365 & -1.03 & 0.3293 & -0.518527 & 0.1916459 \\
\hline$D^{\star} D$ & 0.1810593 & 0.159365 & 1.14 & 0.2824 & -0.174027 & 0.5361459 \\
\hline
\end{tabular}




\section{Method 1 - Lateral Region Stress:}

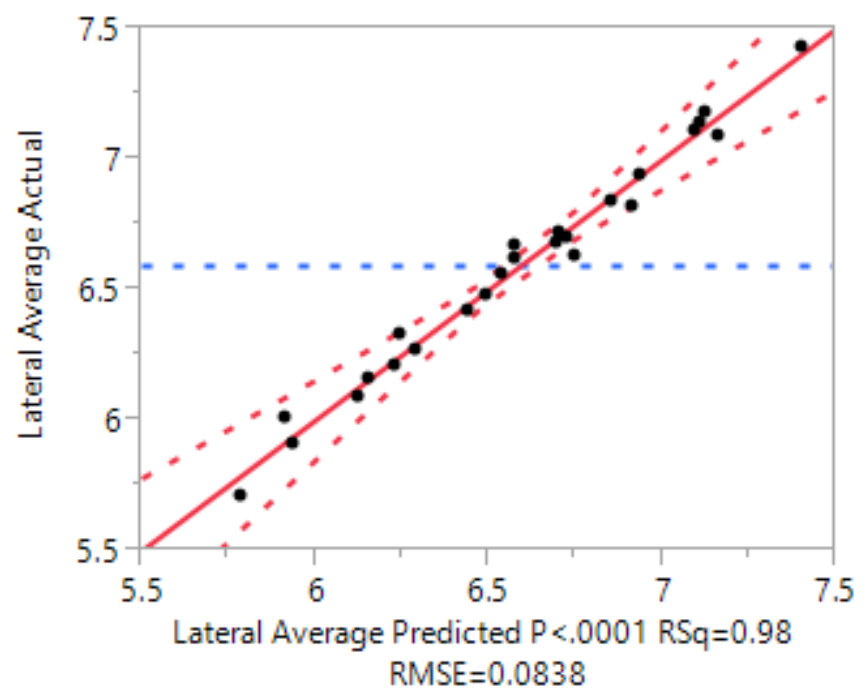

\section{Summary of Fit}

$\begin{array}{lr}\text { RSquare } & 0.984491 \\ \text { RSquare Adj } & 0.962778 \\ \text { Root Mean Square Error } & 0.083769 \\ \text { Mean of Response } & 6.59052 \\ \text { Observations (or Sum Wgts) } & 25\end{array}$

\section{Analysis of Variance}

Sum of

\begin{tabular}{lrrrr|} 
Source & DF & Squares & Mean Square & F Ratio \\
\hline Model & 14 & 4.4544400 & 0.318174 & 45.3419 \\
\hline Error & 10 & 0.0701722 & 0.007017 & Prob > F \\
C. Total & 24 & 4.5246122 & & $<.0001^{*}$
\end{tabular}

\section{Parameter Estimates}

\begin{tabular}{|c|c|c|c|c|c|c|}
\hline Term & Estimate & Std Error & t Ratio & Prob $>|t|$ & Lower 95\% & Upper $95 \%$ \\
\hline Intercept & 6.6977288 & 0.03617 & 185.17 & $<.0001^{*}$ & 6.6171362 & 6.7783214 \\
\hline$A$ & -0.437722 & 0.019745 & -22.17 & $<.0001^{*}$ & -0.481716 & -0.393729 \\
\hline B & 0.0928333 & 0.019745 & 4.70 & $0.0008^{*}$ & 0.0488398 & 0.1368268 \\
\hline C & 0.1688889 & 0.019745 & 8.55 & $<.0001^{*}$ & 0.1248954 & 0.2128824 \\
\hline $\mathrm{D}$ & 0.1078333 & 0.019745 & 5.46 & $0.0003^{*}$ & 0.0638398 & 0.1518268 \\
\hline$A^{\star} B$ & -0.01175 & 0.020942 & -0.56 & 0.5871 & -0.058412 & 0.0349122 \\
\hline$A^{*} \mathrm{C}$ & 0.000875 & 0.020942 & 0.04 & 0.9675 & -0.045787 & 0.0475372 \\
\hline $\mathrm{B}^{\star} \mathrm{C}$ & 0.0015 & 0.020942 & 0.07 & 0.9443 & -0.045162 & 0.0481622 \\
\hline$A^{\star} D$ & -0.031875 & 0.020942 & -1.52 & 0.1590 & -0.078537 & 0.0147872 \\
\hline$B^{\star} \mathrm{D}$ & 0.01475 & 0.020942 & 0.70 & 0.4973 & -0.031912 & 0.0614122 \\
\hline$C^{*} \mathrm{D}$ & -0.015375 & 0.020942 & -0.73 & 0.4797 & -0.062037 & 0.0312872 \\
\hline$A^{*} A$ & -0.02785 & 0.052491 & -0.53 & 0.6073 & -0.144808 & 0.0891079 \\
\hline$B^{\star} B$ & -0.06685 & 0.052491 & -1.27 & 0.2316 & -0.183808 & 0.0501079 \\
\hline $\mathrm{C}^{\star} \mathrm{C}$ & 0.0456497 & 0.052491 & 0.87 & 0.4049 & -0.071308 & 0.1626079 \\
\hline$D^{*} \mathrm{D}$ & -0.09985 & 0.052491 & -1.90 & 0.0863 & -0.216808 & 0.0171079 \\
\hline
\end{tabular}




\section{All Regions Mean Stress Desirability:}

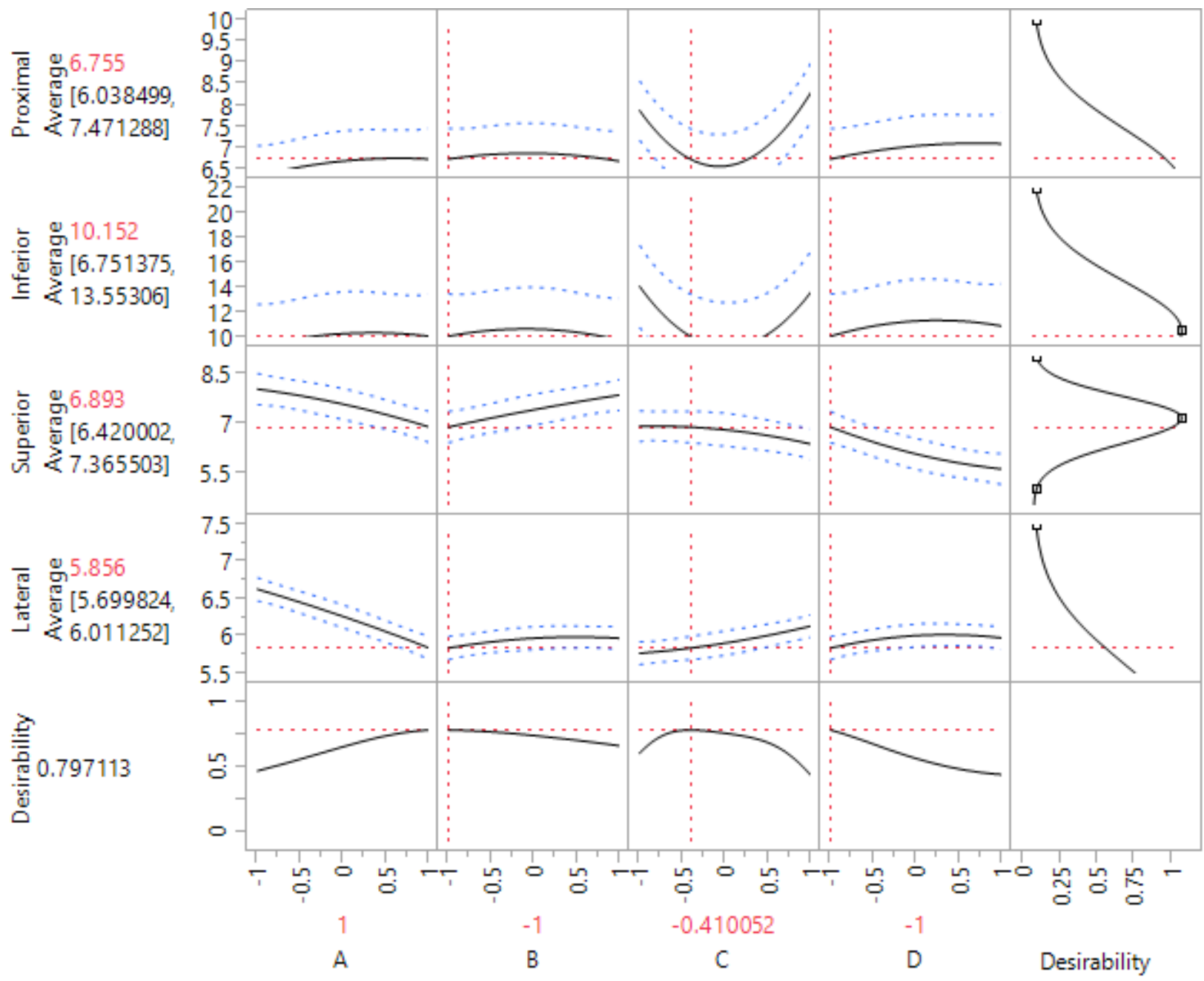




\section{Method 2 - Proximal Region \% of Intact:}

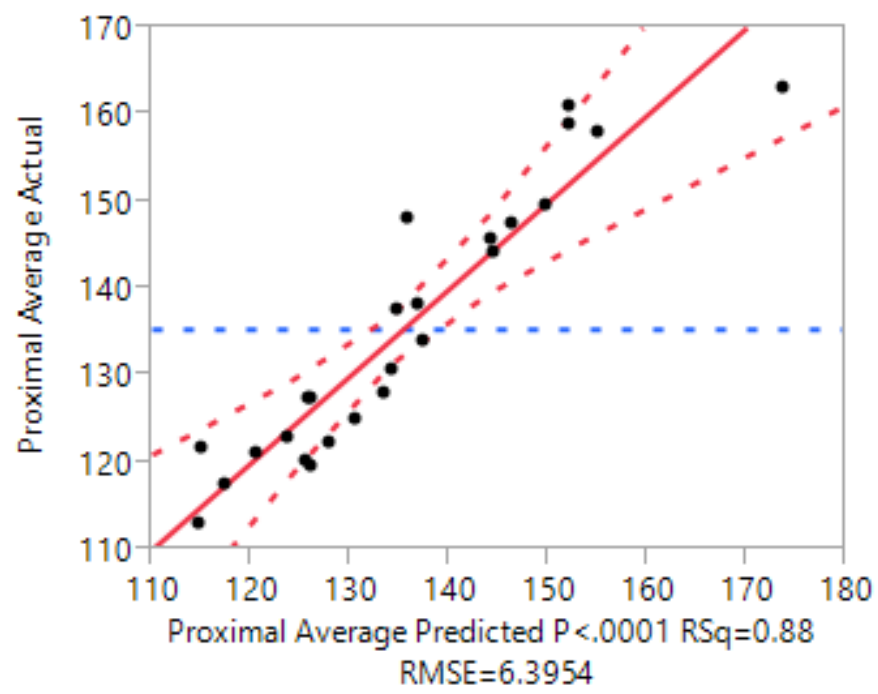

Summary of Fit

\begin{tabular}{|c|c|c|c|c|}
\hline \multicolumn{3}{|l|}{ RSquare } & \multicolumn{2}{|l|}{0.881854} \\
\hline \multicolumn{3}{|c|}{ RSquare Adj } & 0.822781 & \\
\hline \multicolumn{3}{|c|}{ Root Mean Square Error } & 6.395384 & \\
\hline \multicolumn{3}{|c|}{ Mean of Response } & 135.3211 & \\
\hline \multicolumn{3}{|c|}{ Observations (or Sum Wgts) } & 25 & \\
\hline \multicolumn{5}{|c|}{ Analysis of Variance } \\
\hline Source & DF & $\begin{array}{r}\text { Sum of } \\
\text { Squares }\end{array}$ & Mean Square & F Ratio \\
\hline Model & 8 & 4884.6215 & 610.578 & 14.9282 \\
\hline Error & 16 & 654.4150 & 40.901 & Prob $>F$ \\
\hline C. Total & 24 & 5539.0366 & & $<.0001^{*}$ \\
\hline
\end{tabular}

\section{Parameter Estimates}

\begin{tabular}{lrrrrrr}
\hline Term & Estimate & Std Error & t Ratio & Prob $>|\mathbf{t}|$ & Lower 95\% & Upper 95\% \\
Intercept & 120.46051 & 2.417228 & 49.83 & $<.0001^{*}$ & 115.33622 & 125.58481 \\
A & -5.675418 & 1.507406 & -3.77 & $0.0017^{\star}$ & -8.870977 & -2.479859 \\
B & 3.1578305 & 1.507406 & 2.09 & 0.0525 & -0.037729 & 6.3533895 \\
C & 5.3220977 & 1.507406 & 3.53 & $0.0028^{*}$ & 2.1265386 & 8.5176567 \\
D & 5.5409396 & 1.507406 & 3.68 & $0.0020^{*}$ & 2.3453806 & 8.7364986 \\
$A^{*} B$ & -3.574648 & 1.598846 & -2.24 & $0.0400^{*}$ & -6.96405 & -0.185245 \\
$B^{\star} \mathrm{C}$ & 4.0375332 & 1.598846 & 2.53 & $0.0225^{*}$ & 0.648131 & 7.4269354 \\
$A^{*} D$ & -5.306571 & 1.598846 & -3.32 & $0.0043^{*}$ & -8.695973 & -1.917169 \\
$C^{*} \mathrm{C}$ & 20.63971 & 2.848731 & 7.25 & $<.0001^{*}$ & 14.600671 & 26.678749
\end{tabular}




\section{Method 2 - Inferior Region \% of Intact:}

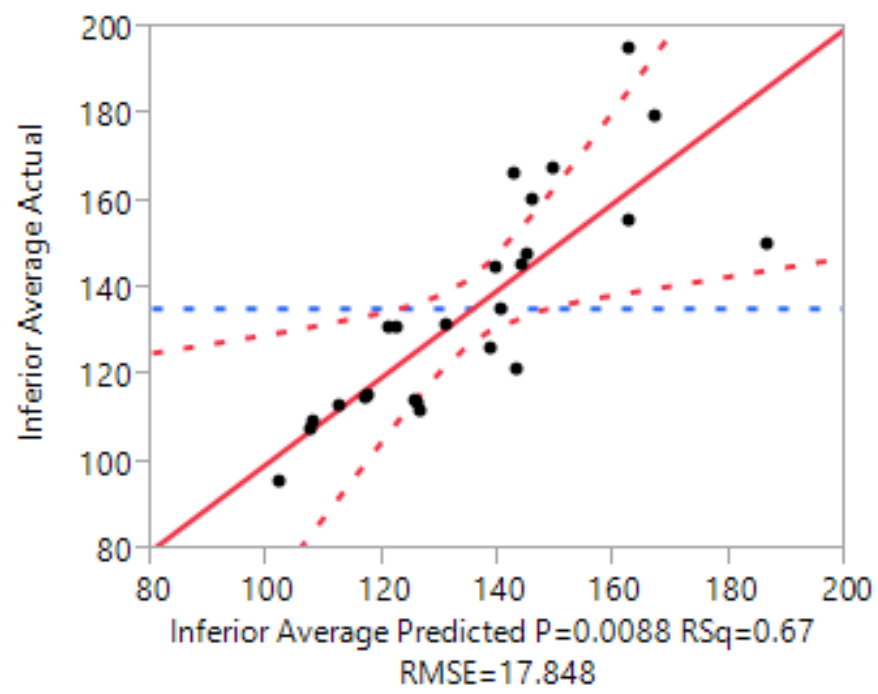

\begin{tabular}{lr}
\hline Summary of Fit & \\
\hline RSquare & 0.666833 \\
RSquare Adj & 0.50025 \\
Root Mean Square Error & 17.84768 \\
Mean of Response & 135.3797 \\
Observations (or Sum Wgts) & 25 \\
\hline
\end{tabular}

Analysis of Variance

\begin{tabular}{|c|c|c|c|c|}
\hline Source & DF & $\begin{array}{l}\text { Sum of } \\
\text { Squares }\end{array}$ & Mean Square & F Ratio \\
\hline Model & 8 & 10200.905 & 1275.11 & 4.0030 \\
\hline Error & 16 & 5096.635 & 318.54 & Prob $>F$ \\
\hline C. Total & 24 & 15297.540 & & $0,0088^{*}$ \\
\hline
\end{tabular}

\section{Parameter Estimates}

\begin{tabular}{lrrrrrr}
\hline Term & Estimate & Std Error & t Ratio & Prob $>|\mathbf{t}|$ & Lower 95\% & Upper 95\% \\
Intercept & 112.4194 & 6.745789 & 16.67 & $<.0001^{*}$ & 98.118967 & 126.71983 \\
A & -10.18306 & 4.206739 & -2.42 & $0.0278^{\star}$ & -19.10095 & -1.265172 \\
B & 4.62477 & 4.206739 & 1.10 & 0.2879 & -4.293117 & 13.542658 \\
C & 1.6101907 & 4.206739 & 0.38 & 0.7069 & -7.307697 & 10.528078 \\
D & 4.8322092 & 4.206739 & 1.15 & 0.2676 & -4.085678 & 13.750097 \\
A $^{\star} \mathrm{B}$ & -5.681824 & 4.46192 & -1.27 & 0.2211 & -15.14067 & 3.7770237 \\
$\mathrm{~B}^{\star} \mathrm{C}$ & 8.0973817 & 4.46192 & 1.81 & 0.0884 & -1.361466 & 17.55623 \\
$\mathrm{~A}^{\star} \mathrm{D}$ & -7.033502 & 4.46192 & -1.58 & 0.1345 & -16.49235 & 2.4253456 \\
$\mathrm{C}^{\star} \mathrm{C}$ & 31.889256 & 7.949989 & 4.01 & $0.0010^{*}$ & 15.036033 & 48.742479
\end{tabular}




\section{Method 2 - Superior Region \% of Intact:}

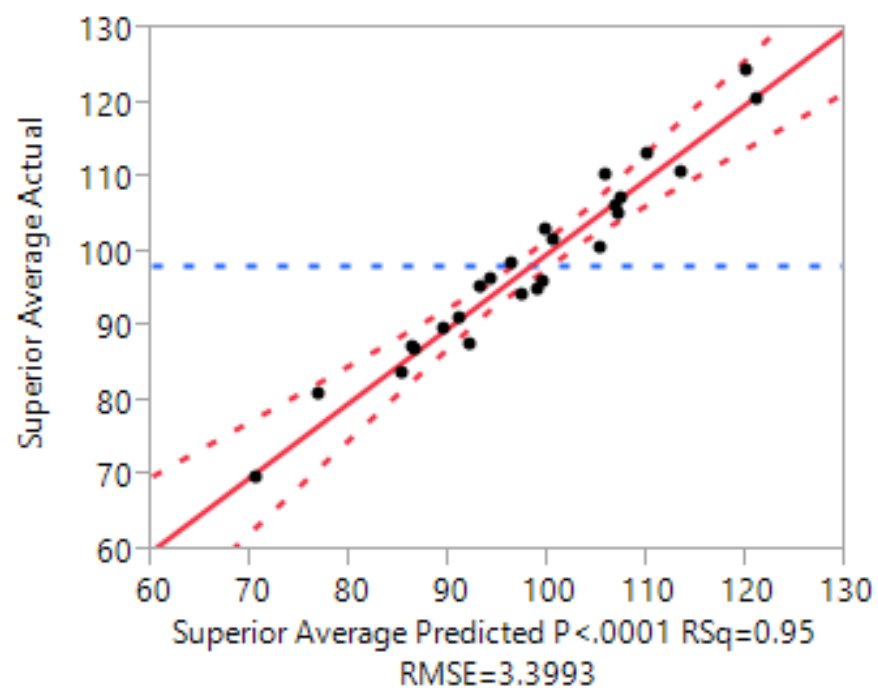

\section{Summary of Fit}

\begin{tabular}{l|r|}
\hline RSquare & 0.951125 \\
RSquare Adj & 0.926688 \\
Root Mean Square Error & 3.399263 \\
Mean of Response & 98.2038 \\
Observations (or Sum Wgts) & 25
\end{tabular}

\section{Analysis of Variance}

\begin{tabular}{|c|c|c|c|c|}
\hline Source & DF & $\begin{array}{l}\text { Sum of } \\
\text { Squares }\end{array}$ & Mean Square & F Ratio \\
\hline Model & 8 & 3597.8466 & 449.731 & 38.9209 \\
\hline Error & 16 & 184.8798 & 11.555 & Prob $>F$ \\
\hline C. Total & 24 & 3782.7264 & & $<.0001^{*}$ \\
\hline
\end{tabular}

\section{Parameter Estimates}

$\begin{array}{lrrrrrr}\text { Term } & \text { Estimate } & \text { Std Error } & \text { t Ratio } & \text { Prob>|t| } & \text { Lower 95\% } & \text { Upper 95\% } \\ \text { Intercept } & 99.696805 & 1.284801 & 77.60 & <.0001^{*} & 96.973149 & 102.42046 \\ \text { A } & -7.614389 & 0.801214 & -9.50 & <.0001^{*} & -9.312887 & -5.915892 \\ \text { B } & 5.570768 & 0.801214 & 6.95 & <.0001^{*} & 3.8722702 & 7.2692657 \\ \text { C } & -1.324214 & 0.801214 & -1.65 & 0.1179 & -3.022712 & 0.3742839 \\ \text { D } & -10.23123 & 0.801214 & -12.77 & <.0001^{*} & -11.92973 & -8.532731 \\ A^{*} B & 0.4389487 & 0.849816 & 0.52 & 0.6126 & -1.36258 & 2.2404776 \\ B^{*} \mathrm{C} & 1.8556427 & 0.849816 & 2.18 & 0.0442^{*} & 0.0541138 & 3.6571716 \\ \text { A }^{*} \mathrm{D} & 0.0199979 & 0.849816 & 0.02 & 0.9815 & -1.781531 & 1.8215268 \\ \mathrm{C}^{*} \mathrm{C} & -2.073619 & 1.514152 & -1.37 & 0.1898 & -5.283478 & 1.1362404\end{array}$




\section{Method 2 - Lateral Region \% of Intact:}

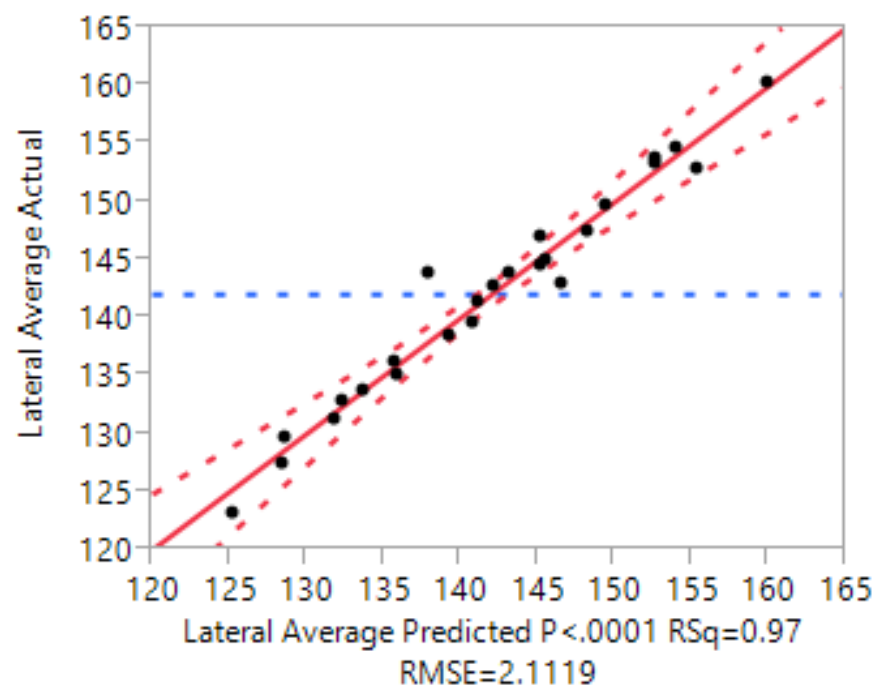

\section{Summary of Fit}

\begin{tabular}{l|r|}
\hline RSquare & 0.966058 \\
\hline RSquare Adj & 0.949087 \\
\hline Root Mean Square Error & 2.111947 \\
\hline Mean of Response & 142.027 \\
\hline Observations (or Sum Wgts) & 25
\end{tabular}

Analysis of Variance

\begin{tabular}{|c|c|c|c|c|}
\hline Source & DF & $\begin{array}{r}\text { Sum of } \\
\text { Squares }\end{array}$ & Mean Square & F Ratio \\
\hline Model & 8 & 2031.1935 & 253.899 & 56.9240 \\
\hline Error & 16 & 71.3651 & 4.460 & Prob $>F$ \\
\hline C. Total & 24 & 2102.5586 & & $<.0001^{*}$ \\
\hline
\end{tabular}

\section{Parameter Estimates}

$\begin{array}{lrrrrrr}\text { Term } & \text { Estimate } & \text { Std Error } & \text { t Ratio } & \text { Prob }>|\mathbf{t}| & \text { Lower 95\% } & \text { Upper 95\% } \\ \text { Intercept } & 143.14207 & 0.798241 & 179.32 & <.0001^{*} & 141.44988 & 144.83427 \\ \text { A } & -9.436322 & 0.497791 & -18.96 & <.0001^{*} & -10.49159 & -8.381053 \\ \text { B } & 1.9991581 & 0.497791 & 4.02 & 0.0010^{*} & 0.943889 & 3.0544272 \\ \text { C } & 3.6398423 & 0.497791 & 7.31 & <.0001^{*} & 2.5845733 & 4.6951114 \\ \text { D } & 2.325121 & 0.497791 & 4.67 & 0.0003^{*} & 1.2698519 & 3.3803901 \\ A^{\star} B & -0.252964 & 0.527987 & -0.48 & 0.6383 & -1.372246 & 0.8663174 \\ B^{\star} \mathrm{C} & 0.0341589 & 0.527987 & 0.06 & 0.9492 & -1.085123 & 1.1534408 \\ A^{\star} \mathrm{D} & -0.686675 & 0.527987 & -1.30 & 0.2118 & -1.805957 & 0.432607 \\ \mathrm{C}^{\star} \mathrm{C} & -1.548713 & 0.940736 & -1.65 & 0.1192 & -3.542984 & 0.445558\end{array}$




\section{All Regions \% of Intact Desirability:}

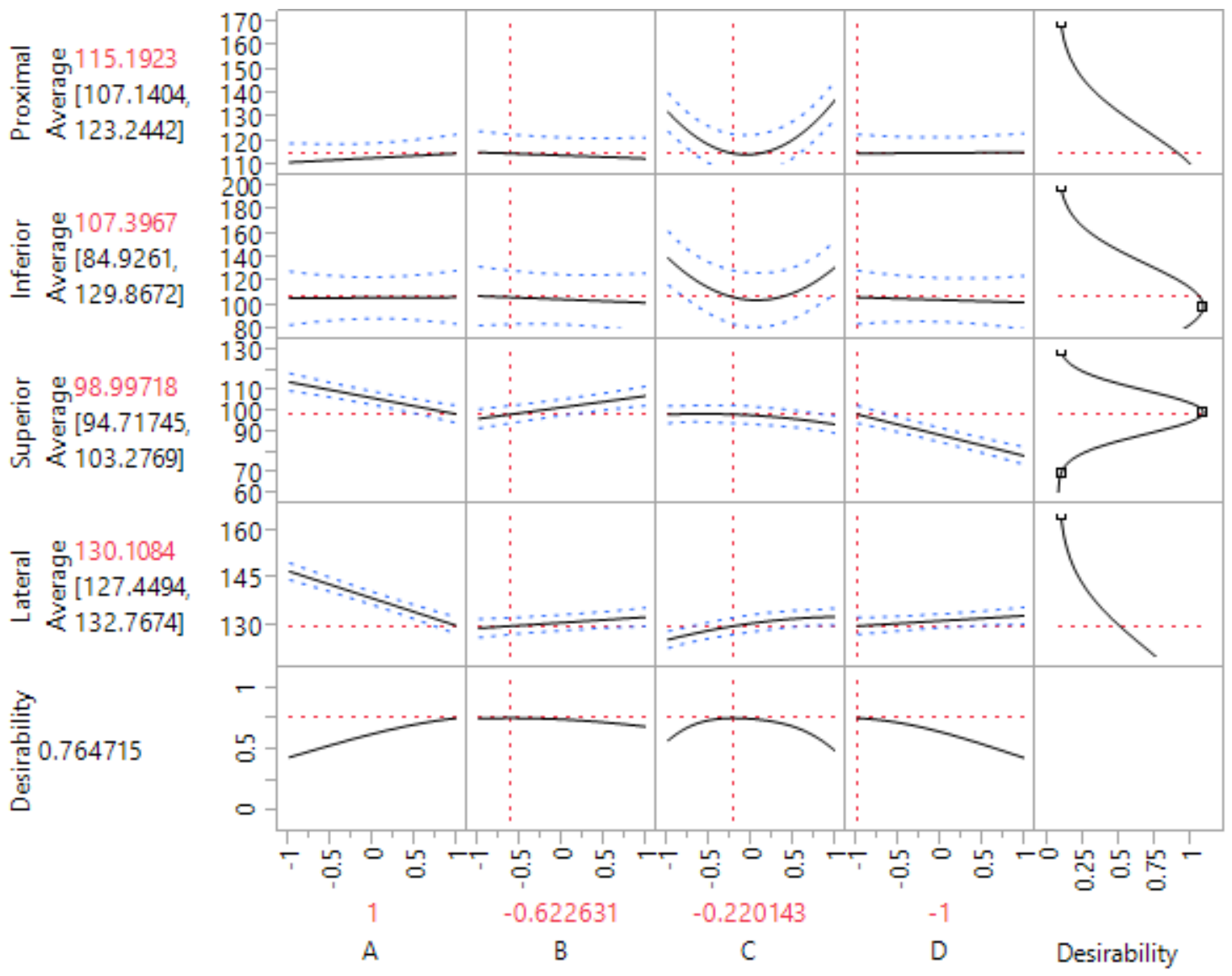




\section{Approach 3 Outcomes - All Regions $90^{\text {th }}$ Percentile}

\section{Method 1 - Proximal Region Stress:}

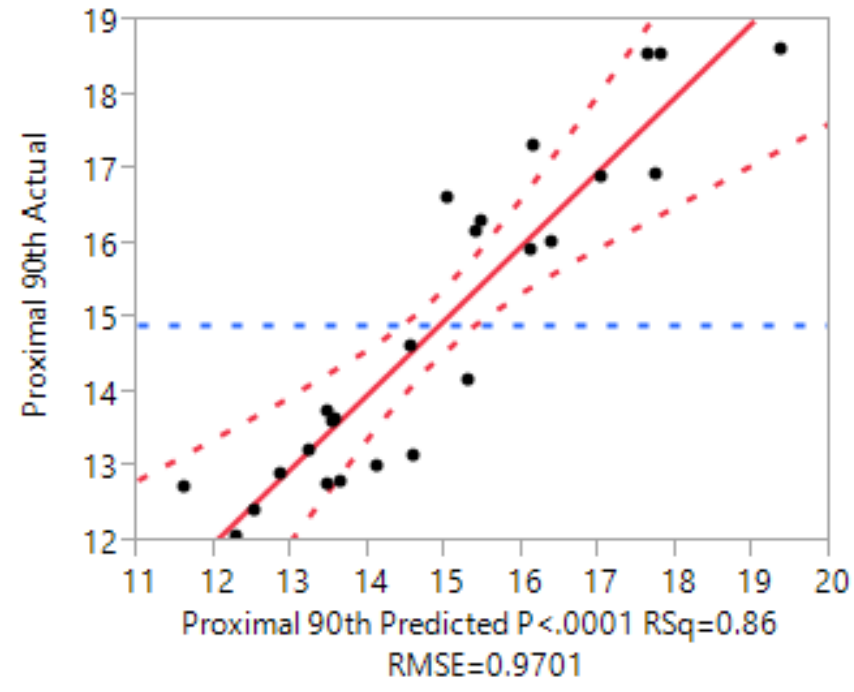

\section{Summary of Fit}

\begin{tabular}{l|r} 
RSquare & 0.862557 \\
RSquare Adj & 0.793835 \\
Root Mean Square Error & 0.970123 \\
Mean of Response & 14.91264 \\
\hline Observations (or Sum Wgts) & 25
\end{tabular}

Analysis of Variance

\begin{tabular}{|c|c|c|c|c|}
\hline Source & DF & $\begin{array}{l}\text { Sum of } \\
\text { Squares }\end{array}$ & Mean Square & F Ratio \\
\hline Model & 8 & 94.50115 & 11.8126 & 12.5515 \\
\hline Error & 16 & 15.05820 & 0.9411 & Prob $>F$ \\
\hline C. Total & 24 & 109.55935 & & $<.0001^{*}$ \\
\hline
\end{tabular}

\section{Parameter Estimates}

$\begin{array}{lrrrrrr}\text { Term } & \text { Estimate } & \text { Std Error } & \text { t Ratio } & \text { Prob }>|\mathbf{t}| & \text { Lower 95\% } & \text { Upper 95\% } \\ \text { Intercept } & 12.87 & 0.366672 & 35.10 & <.0001^{*} & 12.09269 & 13.64731 \\ \text { A } & -0.594222 & 0.22866 & -2.60 & 0.0194^{*} & -1.07896 & -0.109485 \\ \text { B } & 0.3618333 & 0.22866 & 1.58 & 0.1331 & -0.122904 & 0.846571 \\ \text { C } & 0.6735556 & 0.22866 & 2.95 & 0.0095^{*} & 0.1888179 & 1.1582933 \\ \text { D } & 1.2533333 & 0.22866 & 5.48 & <.0001^{*} & 0.7685956 & 1.738071 \\ \mathrm{~B}^{\star} \mathrm{C} & 0.413375 & 0.242531 & 1.70 & 0.1076 & -0.100767 & 0.927517 \\ \mathrm{~A}^{\star} \mathrm{D} & -0.57175 & 0.242531 & -2.36 & 0.0315^{*} & -1.085892 & -0.057608 \\ \mathrm{C}^{\star} \mathrm{D} & -0.226125 & 0.242531 & -0.93 & 0.3650 & -0.740267 & 0.288017 \\ \mathrm{C}^{\star} \mathrm{C} & 2.837 & 0.432127 & 6.57 & <.0001^{*} & 1.9209319 & 3.7530681\end{array}$




\section{Method 1 - Inferior Region Stress:}

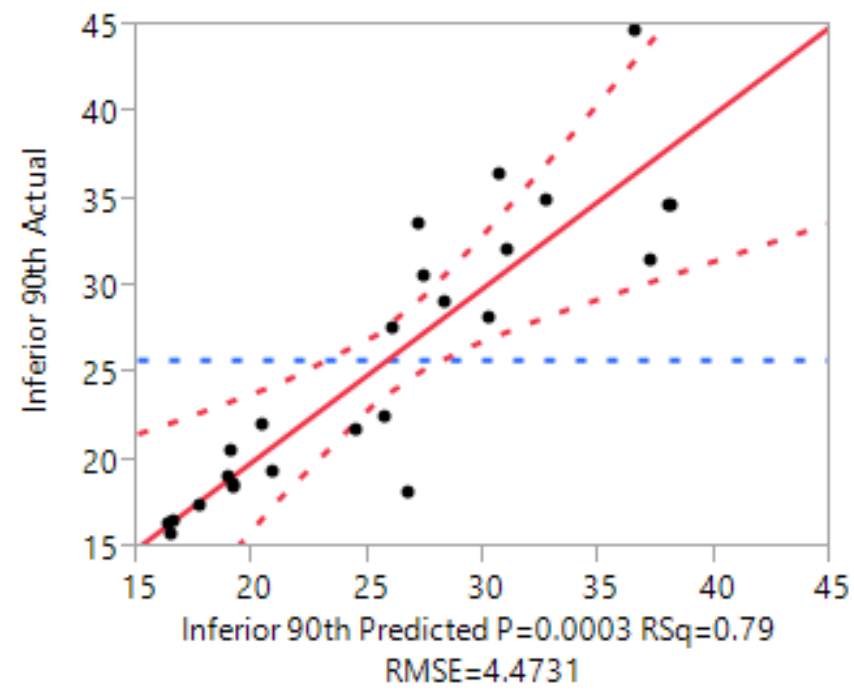

\section{Summary of Fit}

\begin{tabular}{l|r}
\hline RSquare & 0.794707 \\
RSquare Adj & 0.692061 \\
\hline Root Mean Square Error & 4.473087 \\
Mean of Response & 25.7994 \\
Observations (or Sum Wgts) & 25
\end{tabular}

Analysis of Variance

\begin{tabular}{lrrrrr|} 
& & Sum of & & \\
Source & DF & Squares & Mean Square & F Ratio \\
\hline Model & 8 & 1239.2766 & 154.910 & 7.7422 \\
\hline Error & 16 & 320.1361 & 20.009 & Prob > F \\
\hline C. Total & 24 & 1559.4126 & & $0.0003^{*}$
\end{tabular}

\section{Parameter Estimates}

$\begin{array}{lrrrrrr}\text { Term } & \text { Estimate } & \text { Std Error } & \text { t Ratio } & \text { Prob }>\mid \mathbf{t |} & \text { Lower 95\% } & \text { Upper 95\% } \\ \text { Intercept } & 17.757571 & 1.690668 & 10.50 & <.0001^{*} & 14.173516 & 21.341627 \\ \text { A } & -1.341278 & 1.054317 & -1.27 & 0.2215 & -3.576329 & 0.8937737 \\ \text { B } & 1.1553333 & 1.054317 & 1.10 & 0.2894 & -1.079718 & 3.3903848 \\ \text { C } & 1.6946667 & 1.054317 & 1.61 & 0.1275 & -0.540385 & 3.9297181 \\ \text { D } & 1.4048889 & 1.054317 & 1.33 & 0.2014 & -0.830163 & 3.6399403 \\ B^{\star} \mathrm{C} & 3.7716875 & 1.118272 & 3.37 & 0.0039^{*} & 1.4010574 & 6.1423176 \\ A^{\star} \mathrm{D} & -2.152188 & 1.118272 & -1.92 & 0.0723 & -4.522818 & 0.2184426 \\ \mathrm{C}^{\star} \mathrm{D} & -3.213063 & 1.118272 & -2.87 & 0.0110^{*} & -5.583693 & -0.842432 \\ \mathrm{C}^{\star} \mathrm{C} & 11.169206 & 1.992471 & 5.61 & <.0001^{*} & 6.9453561 & 15.393057\end{array}$




\section{Method 1 - Superior Region Stress:}

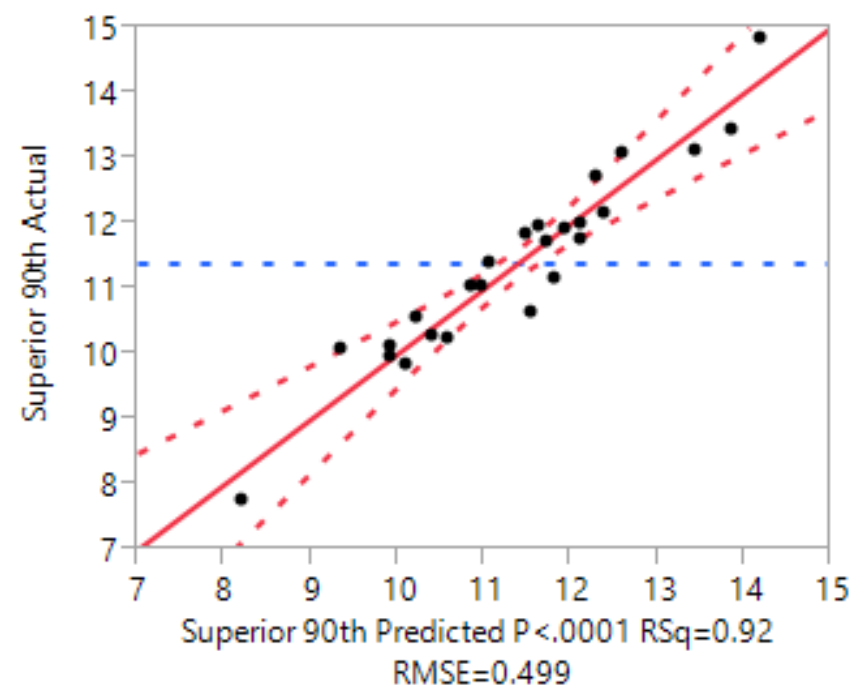

\begin{tabular}{|c|c|c|c|c|}
\hline \multicolumn{4}{|c|}{ Summary of Fit } & \\
\hline \multicolumn{3}{|c|}{ RSquare } & 0.921522 & \\
\hline \multicolumn{3}{|c|}{ RSquare Adj } & 0.882283 & \\
\hline \multicolumn{3}{|c|}{ Root Mean Square Error } & 0.499001 & \\
\hline \multicolumn{3}{|c|}{ Mean of Response } & 11.38644 & \\
\hline \multicolumn{3}{|c|}{ Observations (or Sum Wgts) } & 25 & \\
\hline \multicolumn{5}{|c|}{ Analysis of Variance } \\
\hline \multirow{2}{*}{ Source } & DF & $\begin{array}{l}\text { Sum of } \\
\text { Squares }\end{array}$ & Mean Square & F Ratio \\
\hline & 8 & 46.782087 & 5.84776 & 23.4848 \\
\hline Error & 16 & 3.984030 & 0.24900 & Prob $>F$ \\
\hline C. Total & 24 & 50.766116 & & $<.0001^{*}$ \\
\hline
\end{tabular}

\section{Parameter Estimates}

$\begin{array}{lrrrrrrr}\text { Term } & \text { Estimate } & \text { Std Error } & \text { t Ratio } & \text { Prob }>|\mathbf{t}| & \text { Lower 95\% } & \text { Upper 95\% } \\ \text { Intercept } & 11.483 & 0.188605 & 60.88 & <.0001^{*} & 11.083176 & 11.882824 \\ \text { A } & -0.900778 & 0.117616 & -7.66 & <.0001^{*} & -1.150112 & -0.651444 \\ \text { B } & 0.6197778 & 0.117616 & 5.27 & <.0001^{*} & 0.3704438 & 0.8691118 \\ \text { C } & -0.371778 & 0.117616 & -3.16 & 0.0061^{*} & -0.621112 & -0.122444 \\ \text { D } & -1.097778 & 0.117616 & -9.33 & <.0001^{*} & -1.347112 & -0.848444 \\ B^{\star} \mathrm{C} & 0.2399375 & 0.12475 & 1.92 & 0.0724 & -0.024521 & 0.5043961 \\ A^{\star} D & 0.0509375 & 0.12475 & 0.41 & 0.6885 & -0.213521 & 0.3153961 \\ C^{\star} D & 0.0428125 & 0.12475 & 0.34 & 0.7359 & -0.221646 & 0.3072711 \\ C^{\star} \mathrm{C} & -0.134111 & 0.222273 & -0.60 & 0.5547 & -0.605308 & 0.3370858\end{array}$




\section{Method 1 - Lateral Region Stress:}

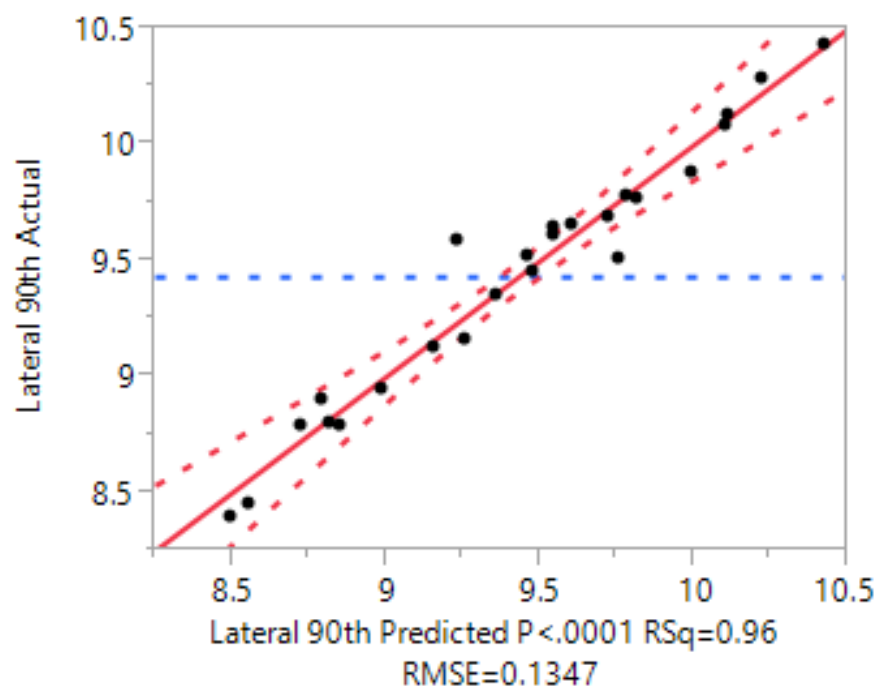

\section{Summary of Fit}

\begin{tabular}{l|r}
\hline RSquare & 0.960098 \\
RSquare Adj & 0.940147 \\
Root Mean Square Error & 0.134682 \\
Mean of Response & 9.43016 \\
Observations (or Sum Wgts) & 25
\end{tabular}

Analysis of Variance

\begin{tabular}{|c|c|c|c|c|}
\hline Source & DF & $\begin{array}{r}\text { Sum of } \\
\text { Squares }\end{array}$ & Mean Square & F Ratio \\
\hline Model & 8 & 6.9833099 & 0.872914 & 48.1227 \\
\hline Error & 16 & 0.2902294 & 0.018139 & Prob $>F$ \\
\hline C. Total & 24 & 7.2735394 & & $<.0001^{*}$ \\
\hline
\end{tabular}

\section{Parameter Estimates}

Term Estimate Std Error t Ratio Prob $>|\mathbf{t}|$ Lower 95\% Upper 95\%

$\begin{array}{lllllll}\text { Intercept } & 9.5408571 & 0.050905 & 187.42 & <.0001^{*} & 9.432943 & 9.6487712\end{array}$

$\begin{array}{lrrrrrr}\text { A } & -0.557667 & 0.031745 & -17.57 & <.0001^{*} & -0.624963 & -0.49037 \\ \text { B } & 0.1826111 & 0.031745 & 5.75 & <.0001^{*} & 0.1153148 & 0.2499074 \\ \text { C } & 0.1585556 & 0.031745 & 4.99 & 0.0001^{*} & 0.0912593 & 0.2258518 \\ \text { D } & 0.0663889 & 0.031745 & 2.09 & 0.0528 & -0.000907 & 0.1336852 \\ B^{\star} \mathrm{C} & 0.0349375 & 0.033671 & 1.04 & 0.3149 & -0.036441 & 0.106316 \\ A^{\star} \mathrm{D} & -0.075438 & 0.033671 & -2.24 & 0.0396^{*} & -0.146816 & -0.004059 \\ C^{\star} \mathrm{D} & -0.038437 & 0.033671 & -1.14 & 0.2704 & -0.109816 & 0.032941 \\ \mathrm{C}^{\star} \mathrm{C} & -0.153746 & 0.059992 & -2.56 & 0.0209^{*} & -0.280924 & -0.026568\end{array}$


All Regions $90^{\text {th }}$ Percentile Stress Desirability:

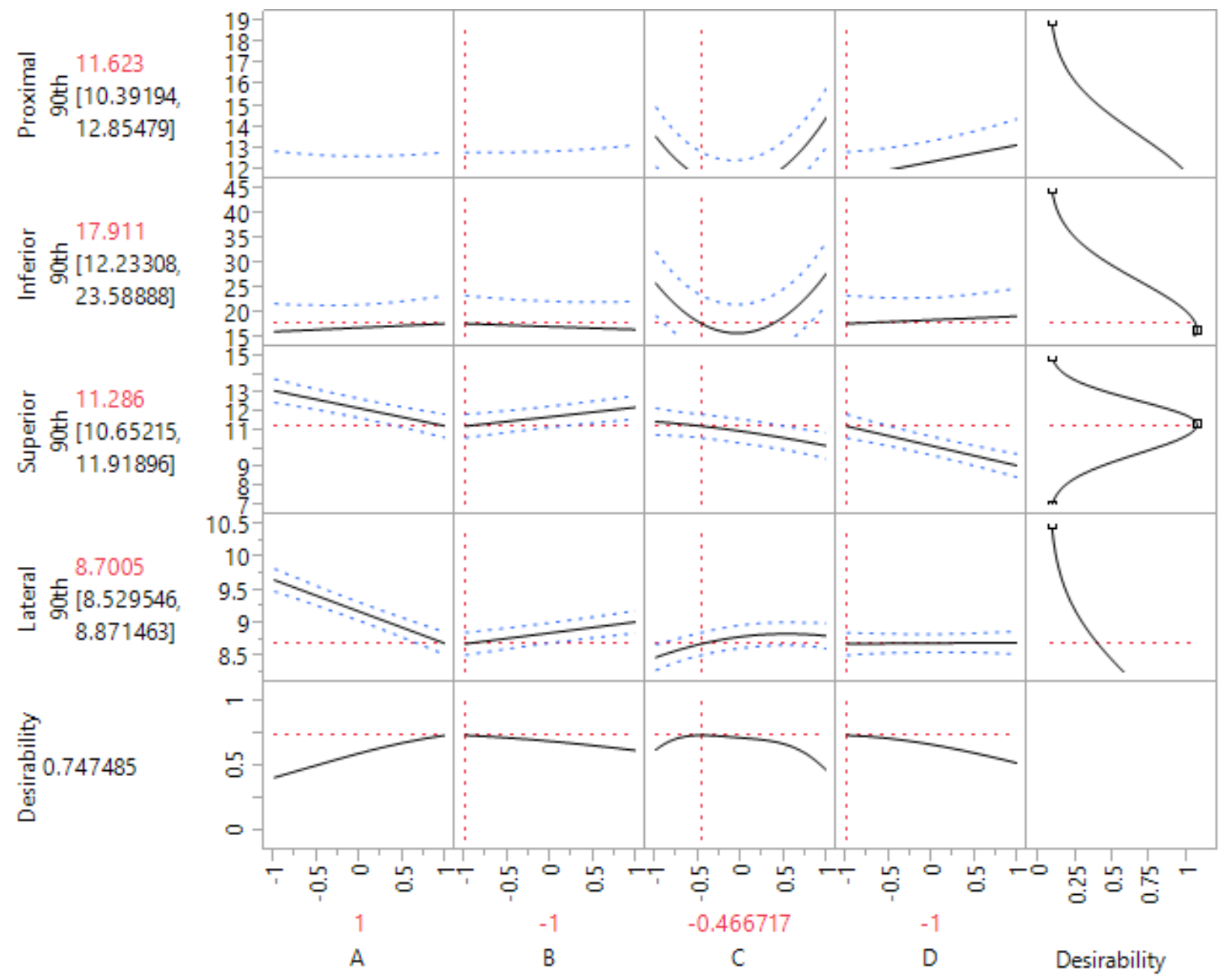




\section{Method 2 - Proximal Region \% of Intact:}

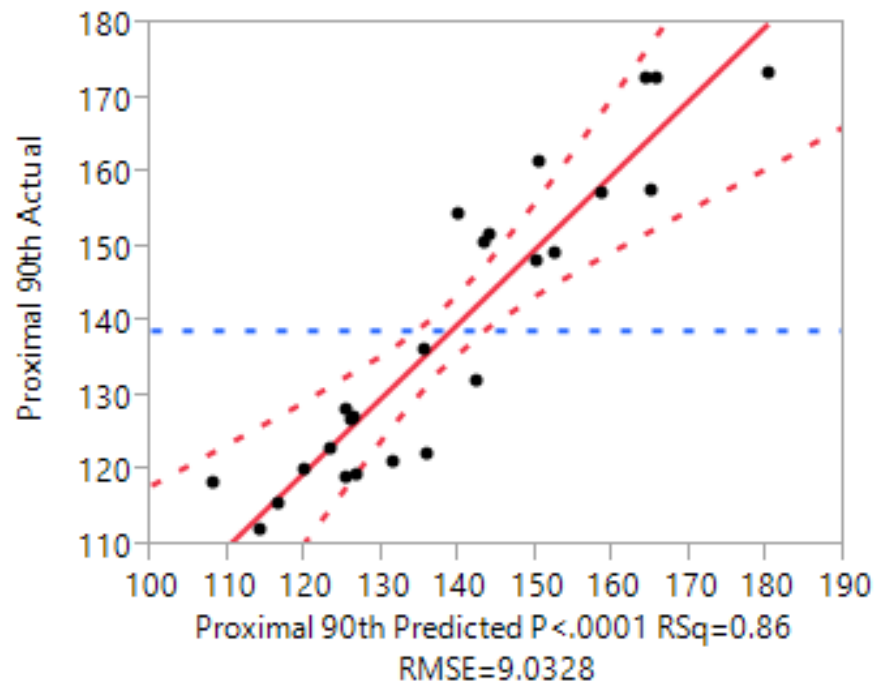

\section{Summary of Fit}

\begin{tabular}{l|r}
\hline RSquare & 0.862554 \\
RSquare Adj & 0.793831 \\
Root Mean Square Error & 9.032808 \\
Mean of Response & 138.8471 \\
\hline Observations (or Sum Wgts) & 25 \\
\hline
\end{tabular}

Analysis of Variance

\begin{tabular}{|c|c|c|c|c|}
\hline Source & DF & $\begin{array}{r}\text { Sum of } \\
\text { Squares }\end{array}$ & Mean Square & F Ratio \\
\hline Model & 8 & 8192.5833 & 1024.07 & 12.5512 \\
\hline Error & 16 & 1305.4659 & 81.59 & Prob $>F$ \\
\hline C. Total & 24 & 9498.0492 & & $<.0001^{*}$ \\
\hline
\end{tabular}

\section{Parameter Estimates}

$\begin{array}{lrrrrrr}\text { Term } & \text { Estimate } & \text { Std Error } & \text { t Ratio } & \text { Prob }>|\mathbf{t}| & \text { Lower 95\% } & \text { Upper 95\% } \\ \text { Intercept } & 119.82892 & 3.41408 & 35.10 & <.0001^{*} & 112.59139 & 127.06645 \\ \text { A } & -5.532796 & 2.129053 & -2.60 & 0.0194^{*} & -10.04619 & -1.019405 \\ \text { B } & 3.3690348 & 2.129053 & 1.58 & 0.1331 & -1.144356 & 7.882426 \\ \text { C } & 6.2721712 & 2.129053 & 2.95 & 0.0095^{*} & 1.75878 & 10.785562 \\ \text { D } & 11.669444 & 2.129053 & 5.48 & <.0001^{*} & 7.1560532 & 16.182836 \\ B^{*} \mathrm{C} & 3.8488799 & 2.258202 & 1.70 & 0.1076 & -0.938294 & 8.6360542 \\ A^{*} \mathrm{D} & -5.324281 & 2.258202 & -2.36 & 0.0314^{*} & -10.11146 & -0.537107 \\ \mathrm{C}^{\star} \mathrm{D} & -2.105446 & 2.258202 & -0.93 & 0.3650 & -6.892621 & 2.681728 \\ \mathrm{C}^{*} \mathrm{C} & 26.4142 & 4.023532 & 6.56 & <.0001^{*} & 17.884692 & 34.943707\end{array}$




\section{Method 2 - Inferior Region \% of Intact:}

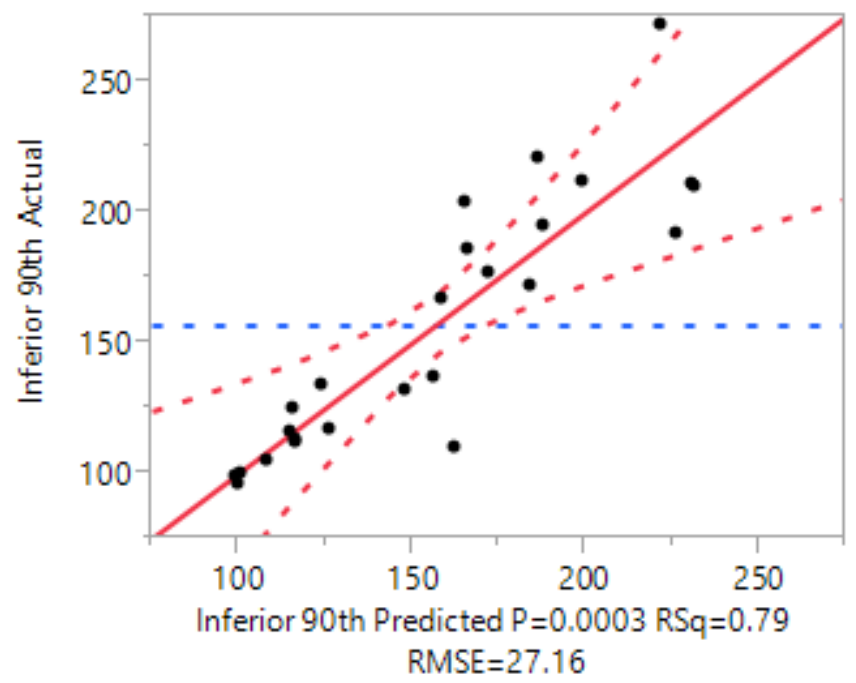

\section{Summary of Fit}

$\begin{array}{lr}\text { RSquare } & 0.7947 \\ \text { RSquare Adj } & 0.69205 \\ \text { Root Mean Square Error } & 27.15992 \\ \text { Mean of Response } & 156.6488 \\ \text { Observations (or Sum Wgts) } & 25\end{array}$

\section{Analysis of Variance}

\begin{tabular}{|c|c|c|c|c|}
\hline Source & DF & $\begin{array}{l}\text { Sum of } \\
\text { Squares }\end{array}$ & Mean Square & F Ratio \\
\hline Model & 8 & 45686.839 & 5710.85 & 7.7418 \\
\hline Error & 16 & 11802.576 & 737.66 & Prob $>F$ \\
\hline C. Total & 24 & 57489.415 & & $0.0003^{*}$ \\
\hline
\end{tabular}

\section{Parameter Estimates}

\begin{tabular}{lrrrrrr} 
Term & Estimate & Std Error & t Ratio & Prob $>|\mathbf{t}|$ & Lower 95\% & Upper 95\% \\
\hline Intercept & 107.82121 & 10.26548 & 10.50 & $<.0001^{*}$ & 86.059359 & 129.58306 \\
A & -8.143707 & 6.401653 & -1.27 & 0.2215 & -21.71461 & 5.4271924 \\
B & 7.0150548 & 6.401653 & 1.10 & 0.2894 & -6.555844 & 20.585954 \\
C & 10.28966 & 6.401653 & 1.61 & 0.1275 & -3.281239 & 23.860559 \\
D & 8.5309208 & 6.401653 & 1.33 & 0.2013 & -5.039978 & 22.10182 \\
$B^{\star} \mathrm{C}$ & 22.900513 & 6.789979 & 3.37 & $0.0039^{*}$ & 8.5064009 & 37.294625 \\
A $^{\star} \mathrm{D}$ & -13.06749 & 6.789979 & -1.92 & 0.0723 & -27.4616 & 1.3266237 \\
$\mathrm{C}^{\star} \mathrm{D}$ & -19.50864 & 6.789979 & -2.87 & $0.0110^{*}$ & -33.90275 & -5.114525 \\
$\mathrm{C}^{\star} \mathrm{C}$ & 67.816045 & 12.09799 & 5.61 & $<.0001^{*}$ & 42.169456 & 93.462633
\end{tabular}




\section{Method 2 - Superior Region \% of Intact:}

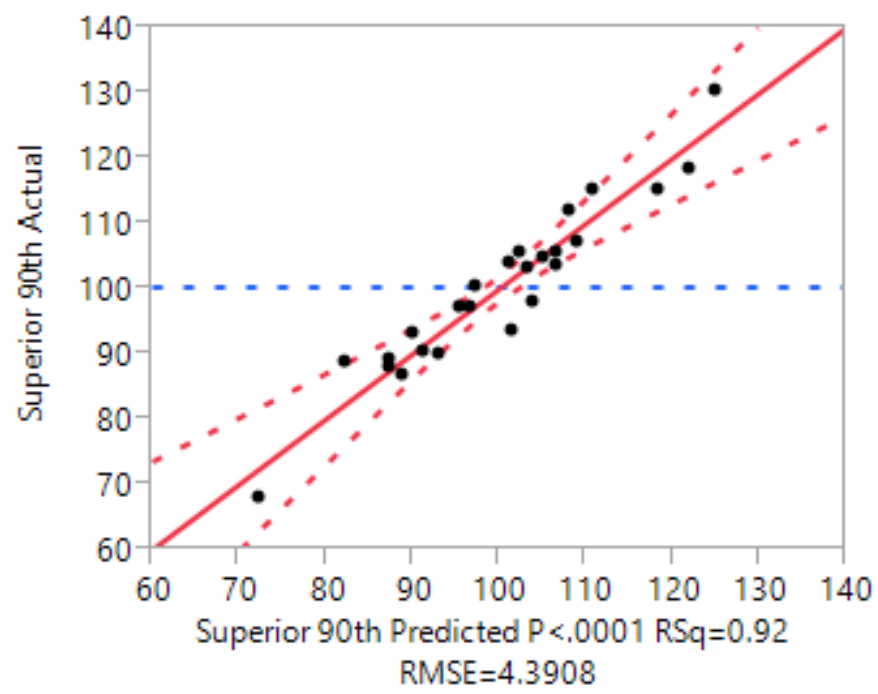

\begin{tabular}{|lr|}
\hline Summary of Fit & \\
\hline RSquare & 0.921578 \\
\hline RSquare Adj & 0.882367 \\
\hline Root Mean Square Error & 4.390826 \\
\hline Mean of Response & 100.2198 \\
\hline Observations (or Sum Wgts) & 25 \\
\hline
\end{tabular}

\begin{tabular}{l|r|r|r|r|}
\hline \multicolumn{7}{l}{ Analysis of Variance } \\
Source & DF & $\begin{array}{r}\text { Sum of } \\
\text { Squares }\end{array}$ & Mean Square & F Ratio \\
Model & 8 & 3624.9734 & 453.122 & 23.5030 \\
Error & 16 & 308.4696 & 19.279 & Prob > F \\
C. Total & 24 & 3933.4430 & & $<.0001^{*}$
\end{tabular}

\section{Parameter Estimates}

\begin{tabular}{lrrrrrr} 
Term & Estimate & Std Error & t Ratio & Prob $>$ |t| & Lower 95\% & Upper 95\% \\
\hline Intercept & 101.07004 & 1.659576 & 60.90 & $<.0001^{*}$ & 97.551895 & 104.58818 \\
A & -7.929001 & 1.034928 & -7.66 & $<.0001^{*}$ & -10.12295 & -5.735053 \\
B & 5.4550202 & 1.034928 & 5.27 & $<.0001^{*}$ & 3.2610717 & 7.6489687 \\
C & -3.273169 & 1.034928 & -3.16 & $0.0060^{*}$ & -5.467117 & -1.07922 \\
D & -9.663764 & 1.034928 & -9.34 & $<.0001^{*}$ & -11.85771 & -7.469816 \\
$B^{\star} \mathrm{C}$ & 2.1115355 & 1.097706 & 1.92 & 0.0724 & -0.215498 & 4.4385693 \\
A $^{*} \mathrm{D}$ & 0.4487673 & 1.097706 & 0.41 & 0.6881 & -1.878266 & 2.7758011 \\
$\mathrm{C}^{\star} \mathrm{D}$ & 0.3771542 & 1.097706 & 0.34 & 0.7356 & -1.94988 & 2.704188 \\
$\mathrm{C}^{\star} \mathrm{C}$ & -1.180941 & 1.955829 & -0.60 & 0.5544 & -5.327114 & 2.965232
\end{tabular}




\section{Method 2 - Lateral Region \% of Intact:}

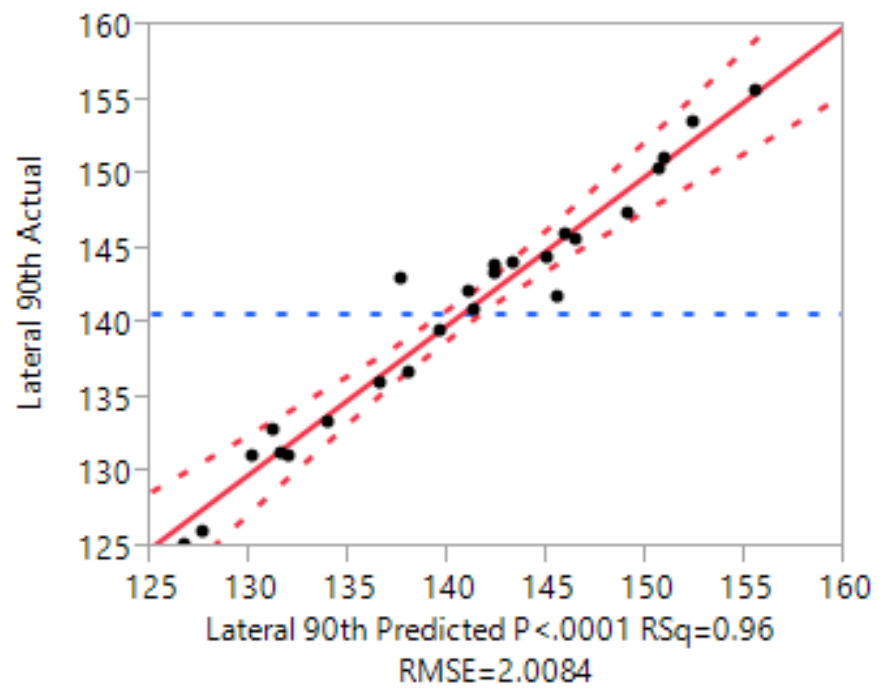

\section{Summary of Fit}

\begin{tabular}{l|r}
\hline RSquare & 0.96013 \\
RSquare Adj & 0.940195 \\
Root Mean Square Error & 2.008354 \\
Mean of Response & 140.6806 \\
Observations (or Sum Wgts) & 25
\end{tabular}

\section{Analysis of Variance}

Sum of

\begin{tabular}{lrrrrr|} 
Source & DF & Squares & Mean Square & F Ratio \\
\hline Model & 8 & 1554.1151 & 194.264 & 48.1629 \\
\hline Error & 16 & 64.5358 & 4.033 & Prob > F \\
C. Total & 24 & 1618.6509 & & $<.0001^{*}$
\end{tabular}

\section{Parameter Estimates}

\begin{tabular}{lrrrrrr} 
Term & Estimate & Std Error & t Ratio & Prob $>|\mathbf{t}|$ & Lower 95\% & Upper 95\% \\
\hline Intercept & 142.33183 & 0.759086 & 187.50 & $<.0001^{*}$ & 140.72264 & 143.94102 \\
A & -8.319758 & 0.473374 & -17.58 & $<.0001^{*}$ & -9.323265 & -7.316251 \\
B & 2.7252738 & 0.473374 & 5.76 & $<.0001^{*}$ & 1.7217668 & 3.7287808 \\
C & 2.3630256 & 0.473374 & 4.99 & $0.0001^{*}$ & 1.3595186 & 3.3665326 \\
D & 0.9896931 & 0.473374 & 2.09 & 0.0529 & -0.013814 & 1.9932001 \\
$B^{\star} \mathrm{C}$ & 0.5220268 & 0.502088 & 1.04 & 0.3139 & -0.542353 & 1.5864067 \\
$A^{*} \mathrm{D}$ & -1.12519 & 0.502088 & -2.24 & $0.0396^{*}$ & -2.18957 & -0.06081 \\
$\mathrm{C}^{\star} \mathrm{D}$ & -0.571816 & 0.502088 & -1.14 & 0.2715 & -1.636196 & 0.4925635 \\
$\mathrm{C}^{\star} \mathrm{C}$ & -2.293315 & 0.894592 & -2.56 & $0.0208^{*}$ & -4.189765 & -0.396865
\end{tabular}


All Regions $90^{\text {th }}$ Percentile $\%$ of Intact Desirability:

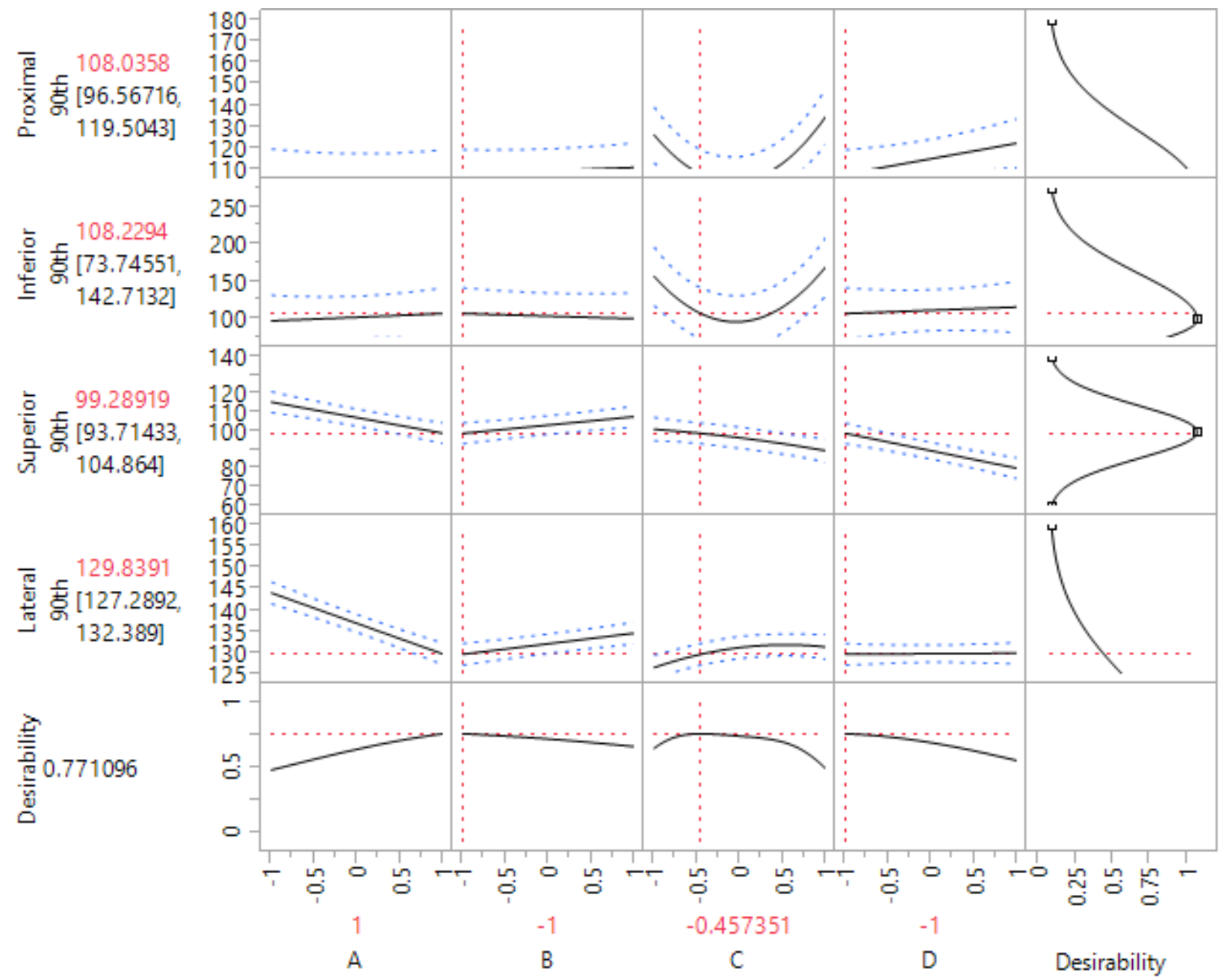




\section{Appendix E: Complete Flexion Phase DOE Results Approach 1 Outcomes - Proximal Region}

\section{Method 1 - Mean Stress Model:}

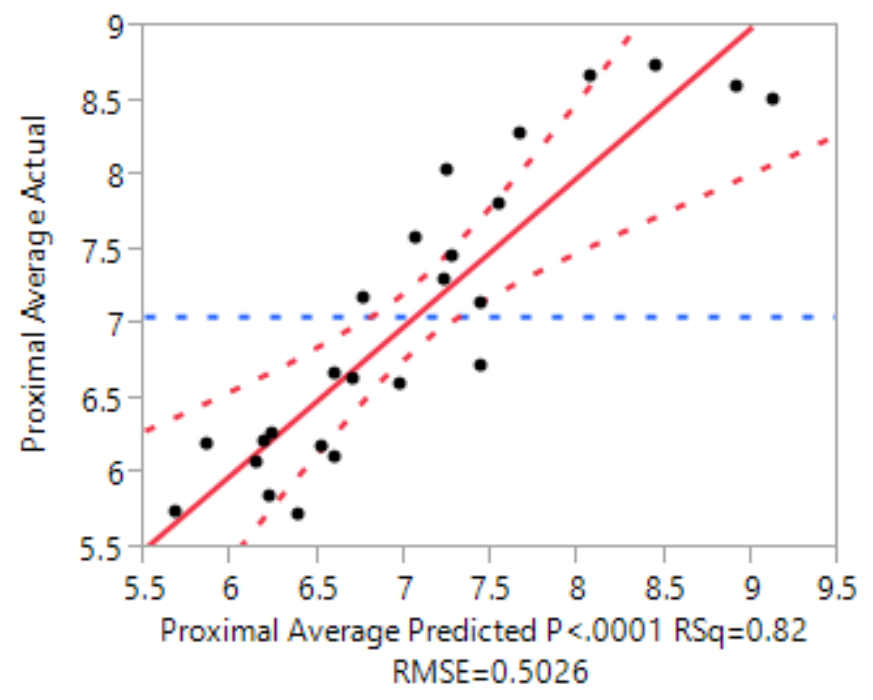

\begin{tabular}{|c|c|c|c|c|}
\hline \multicolumn{4}{|c|}{ Summary of Fit } & \\
\hline \multicolumn{3}{|l|}{ RSquare } & 0.818672 & \\
\hline \multicolumn{3}{|c|}{ RSquare Adj } & 0.744008 & \\
\hline \multicolumn{3}{|c|}{ Root Mean Square Error } & 0.5026 & \\
\hline \multicolumn{3}{|c|}{ Mean of Response } & 7.05376 & \\
\hline \multicolumn{3}{|c|}{ Observations (or Sum Wgts) } & 25 & \\
\hline \multicolumn{5}{|c|}{ Analysis of Variance } \\
\hline Source & DF & $\begin{array}{r}\text { Sum of } \\
\text { Squares }\end{array}$ & Mean Square & F Ratio \\
\hline Model & 7 & 19.388319 & 2.76976 & 10.9647 \\
\hline Error & 17 & 4.294318 & 0.25261 & Prob $>F$ \\
\hline C. Total & 24 & 23.682637 & & $<.0001^{*}$ \\
\hline
\end{tabular}

\section{Parameter Estimates}

$\begin{array}{lrrrrrr}\text { Term } & \text { Estimate } & \text { Std Error } & \text { t Ratio } & \text { Prob }>|\mathbf{t}| & \text { Lower 95\% } & \text { Upper 95\% } \\ \text { Intercept } & 6.1922857 & 0.189965 & 32.60 & <.0001^{*} & 5.7914946 & 6.5930768 \\ \text { A } & -0.517389 & 0.118464 & -4.37 & 0.0004^{*} & -0.767326 & -0.267452 \\ \text { B } & -0.041278 & 0.118464 & -0.35 & 0.7318 & -0.291215 & 0.2086594 \\ \text { C } & 0.1491111 & 0.118464 & 1.26 & 0.2251 & -0.100826 & 0.3990483 \\ \text { D } & 0.3265556 & 0.118464 & 2.76 & 0.0135^{*} & 0.0766184 & 0.5764927 \\ B^{\star} \mathrm{C} & 0.3806875 & 0.12565 & 3.03 & 0.0076^{*} & 0.1155891 & 0.6457859 \\ A^{*} \mathrm{D} & -0.409687 & 0.12565 & -3.26 & 0.0046^{*} & -0.674786 & -0.144589 \\ \mathrm{C}^{*} \mathrm{C} & 1.1964921 & 0.223876 & 5.34 & <.0001^{*} & 0.7241552 & 1.6688289\end{array}$




\section{Method 1 - Stress Standard Deviation Model:}

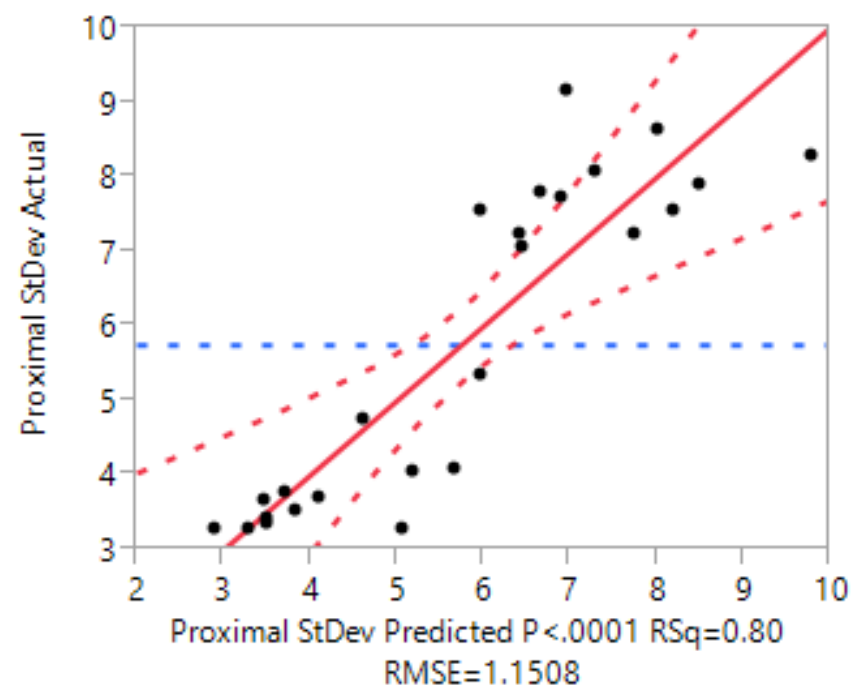

\section{Summary of Fit}

\begin{tabular}{l|r}
\hline RSquare & 0.796593 \\
RSquare Adj & 0.712837 \\
Root Mean Square Error & 1.150784 \\
Mean of Response & 5.74608 \\
Observations (or Sum Wgts) & 25
\end{tabular}

Analysis of Variance

\begin{tabular}{lrrrrr|} 
& & Sum of & & \\
Source & DF & Squares & Mean Square & F Ratio \\
\hline Model & 7 & 88.16692 & 12.5953 & 9.5109 \\
\hline Error & 17 & 22.51315 & 1.3243 & Prob $>$ F \\
\hline C. Total & 24 & 110.68007 & & $<.0001^{*}$
\end{tabular}

\section{Parameter Estimates}

$\begin{array}{lrrrrrr}\text { Term } & \text { Estimate } & \text { Std Error } & \text { t Ratio } & \text { Prob }>|\mathbf{t}| & \text { Lower 95\% } & \text { Upper 95\% } \\ \text { Intercept } & 3.4944286 & 0.434955 & 8.03 & <.0001^{*} & 2.576753 & 4.4121041 \\ \text { A } & -0.207 & 0.271242 & -0.76 & 0.4558 & -0.779271 & 0.3652713 \\ \text { B } & -0.015611 & 0.271242 & -0.06 & 0.9548 & -0.587882 & 0.5566602 \\ \text { C } & 0.6587222 & 0.271242 & 2.43 & 0.0265^{*} & 0.086451 & 1.2309935 \\ \text { D } & 0.5986111 & 0.271242 & 2.21 & 0.0414^{\star} & 0.0263398 & 1.1708824 \\ B^{\star} \mathrm{C} & 0.9011875 & 0.287696 & 3.13 & 0.0061^{*} & 0.2942022 & 1.5081728 \\ A^{\star} \mathrm{D} & -0.823313 & 0.287696 & -2.86 & 0.0108^{\star} & -1.430298 & -0.216327 \\ \mathrm{C}^{\star} \mathrm{C} & 3.1272937 & 0.5126 & 6.10 & <.0001^{*} & 2.0458026 & 4.2087847\end{array}$




\section{Method $1-90^{\text {th }}$ Percentile Stress Model:}

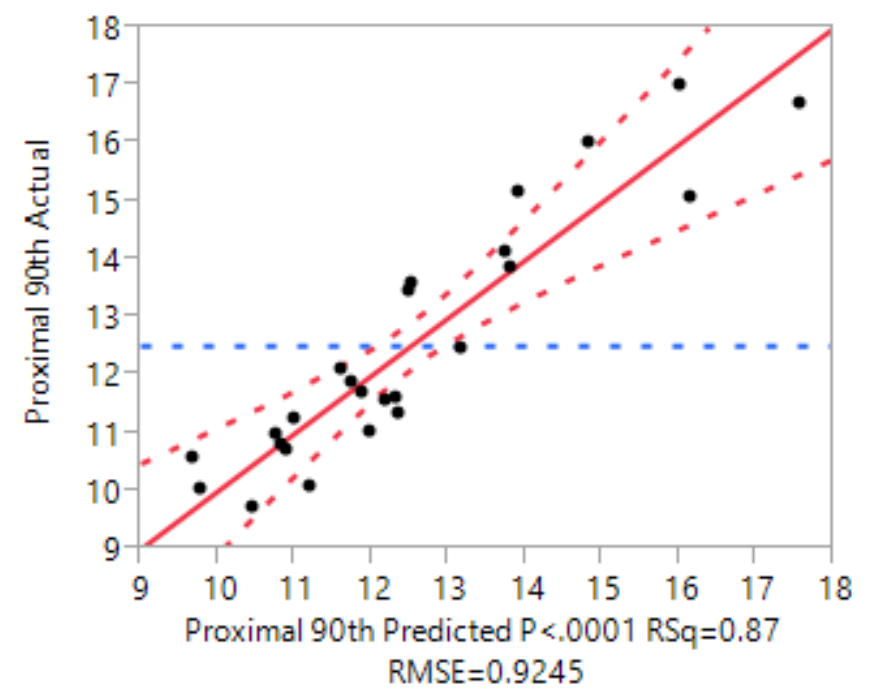

\section{Summary of Fit}

\begin{tabular}{lr}
\hline RSquare & 0.868507 \\
RSquare Adj & 0.814362 \\
Root Mean Square Error & 0.924466 \\
Mean of Response & 12.50812 \\
Observations (or Sum Wgts) & 25
\end{tabular}

\section{Analysis of Variance}

\begin{tabular}{|c|c|c|c|c|}
\hline Source & DF & $\begin{array}{l}\text { Sum of } \\
\text { Squares }\end{array}$ & Mean Square & F Ratio \\
\hline Model & 7 & 95.96203 & 13.7089 & 16.0406 \\
\hline Error & 17 & 14.52883 & 0.8546 & Prob $>F$ \\
\hline C. Total & 24 & 110.49085 & & $<.0001^{*}$ \\
\hline
\end{tabular}

\section{'Parameter Estimates}

$\begin{array}{lrrrrrr}\text { Term } & \text { Estimate } & \text { Std Error } & \text { t Ratio } & \text { Prob }>|\mathbf{t}| & \text { Lower 95\% } & \text { Upper 95\% } \\ \text { Intercept } & 10.829286 & 0.349415 & 30.99 & <.0001^{*} & 10.092084 & 11.566487 \\ \text { A } & -1.05 & 0.217899 & -4.82 & 0.0002^{*} & -1.509726 & -0.590274 \\ \text { B } & 0.0655 & 0.217899 & 0.30 & 0.7674 & -0.394226 & 0.525226 \\ \text { C } & 0.6468889 & 0.217899 & 2.97 & 0.0086^{*} & 0.1871629 & 1.1066149 \\ \text { D } & 1.1401667 & 0.217899 & 5.23 & <.0001^{*} & 0.6804407 & 1.5998926 \\ \text { B }^{\star} \mathrm{C} & 0.715125 & 0.231116 & 3.09 & 0.0066^{*} & 0.227512 & 1.202738 \\ \text { A D }^{\star} & -0.7715 & 0.231116 & -3.34 & 0.0039^{\star} & -1.259113 & -0.283887 \\ \text { C }^{\star} \mathrm{C} & 2.3317143 & 0.41179 & 5.66 & <.0001^{*} & 1.4629139 & 3.2005147\end{array}$




\section{Proximal Region Stress Desirability:}

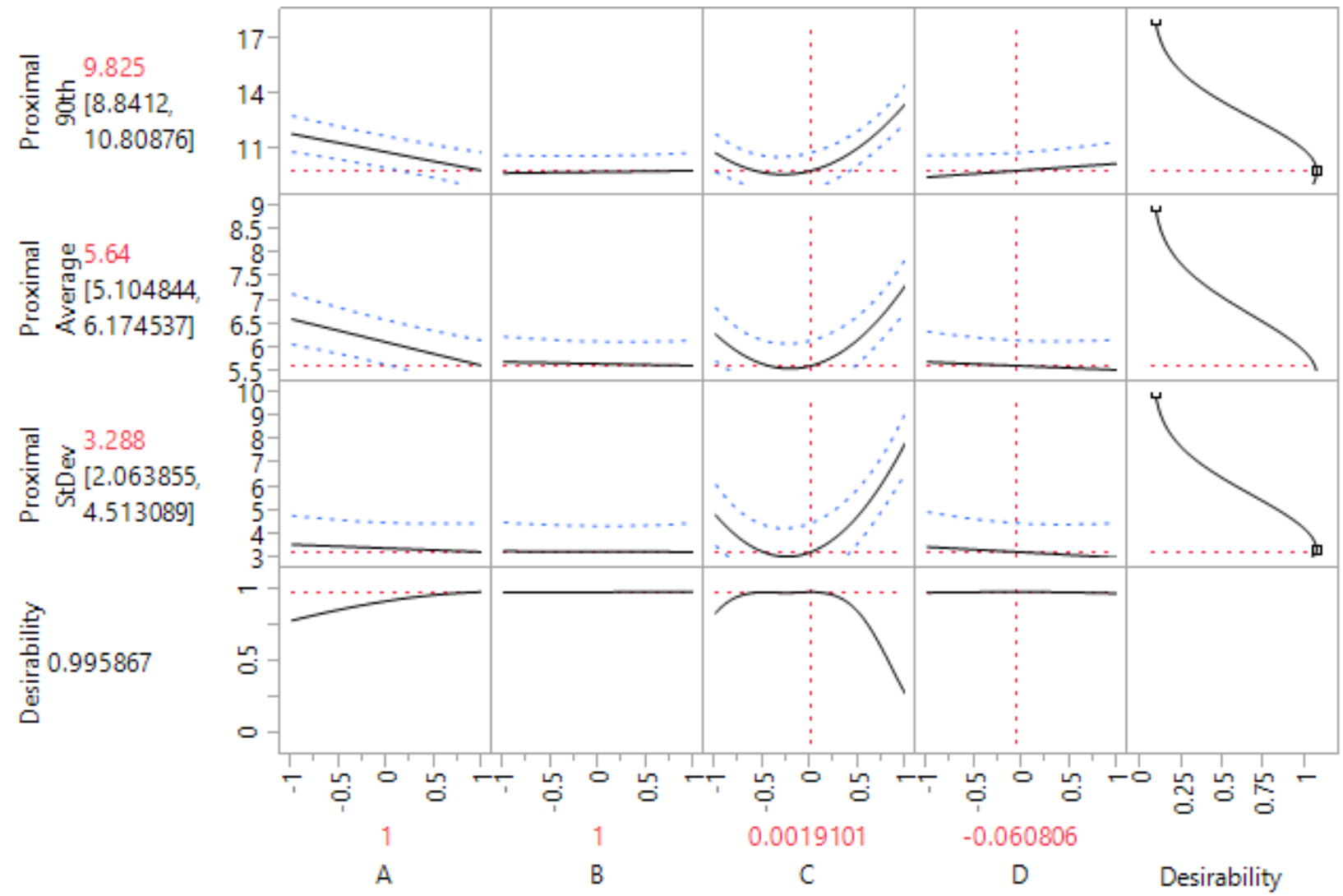




\section{Method 2 - Mean \% of Intact Stress Model:}

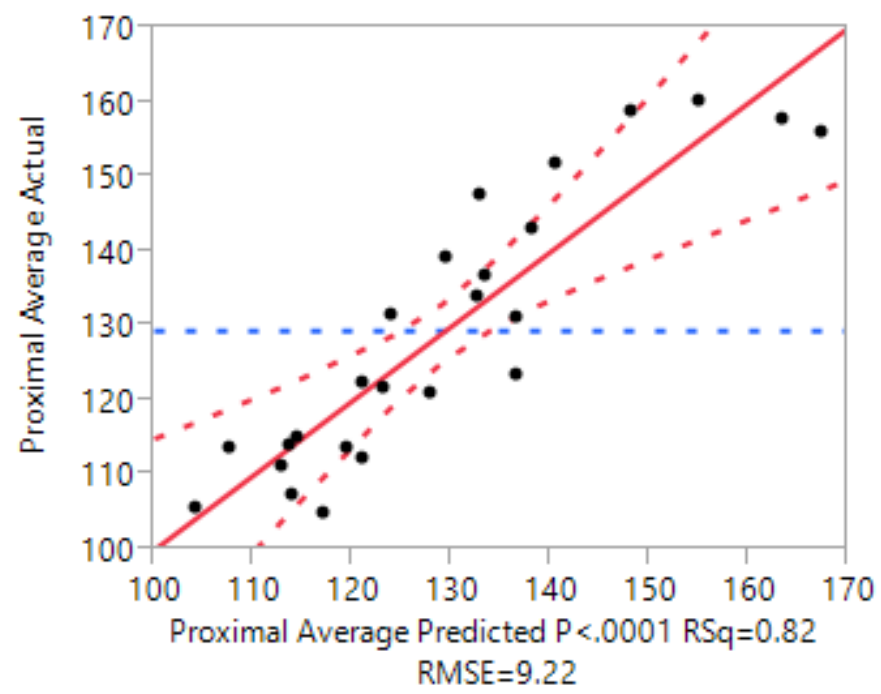

\section{Summary of Fit}

\begin{tabular}{|c|c|c|c|c|}
\hline \multicolumn{3}{|c|}{ RSquare } & \multicolumn{2}{|l|}{0.818626} \\
\hline \multicolumn{3}{|c|}{ RSquare Adj } & \multicolumn{2}{|l|}{0.743942} \\
\hline \multicolumn{3}{|c|}{ Root Mean Square Error } & \multicolumn{2}{|l|}{9.219953} \\
\hline \multicolumn{3}{|c|}{ Mean of Response } & \multicolumn{2}{|l|}{129.3869} \\
\hline \multicolumn{3}{|c|}{ Observations (or Sum Wgts) } & \multicolumn{2}{|l|}{25} \\
\hline \multicolumn{5}{|c|}{ Analysis of Variance } \\
\hline Source & DF & $\begin{array}{l}\text { Sum of } \\
\text { Squares }\end{array}$ & Mean Square & F Ratio \\
\hline Model & 7 & 6522.5195 & 931.789 & 10.9612 \\
\hline Error & 17 & 1445.1280 & 85.008 & Prob $>F$ \\
\hline C. Total & 24 & 7967.6475 & & $<.0001^{*}$ \\
\hline
\end{tabular}

\section{Parameter Estimates}

$\begin{array}{lrrrrrr}\text { Term } & \text { Estimate } & \text { Std Error } & \text { t Ratio } & \text { Prob }>|\mathbf{t}| & \text { Lower 95\% } & \text { Upper 95\% } \\ \text { Intercept } & 113.58719 & 3.484815 & 32.59 & <.0001^{*} & 106.23487 & 120.93951 \\ \text { A } & -9.488682 & 2.173164 & -4.37 & 0.0004^{*} & -14.07366 & -4.903707 \\ \text { B } & -0.757854 & 2.173164 & -0.35 & 0.7316 & -5.342829 & 3.8271203 \\ \text { C } & 2.7334636 & 2.173164 & 1.26 & 0.2255 & -1.851511 & 7.3184381 \\ \text { D } & 5.9894365 & 2.173164 & 2.76 & 0.0135^{*} & 1.404462 & 10.574411 \\ B^{\star} \mathrm{C} & 6.9829582 & 2.304988 & 3.03 & 0.0076^{*} & 2.1198583 & 11.846058 \\ \text { A }^{*} & -7.517357 & 2.304988 & -3.26 & 0.0046^{*} & -12.38046 & -2.654257 \\ \text { C }^{\star} \mathrm{C} & 21.944098 & 4.106893 & 5.34 & <.0001^{*} & 13.279311 & 30.608886\end{array}$




\section{Method 2 - \% of Intact Stress Standard Deviation Model:}

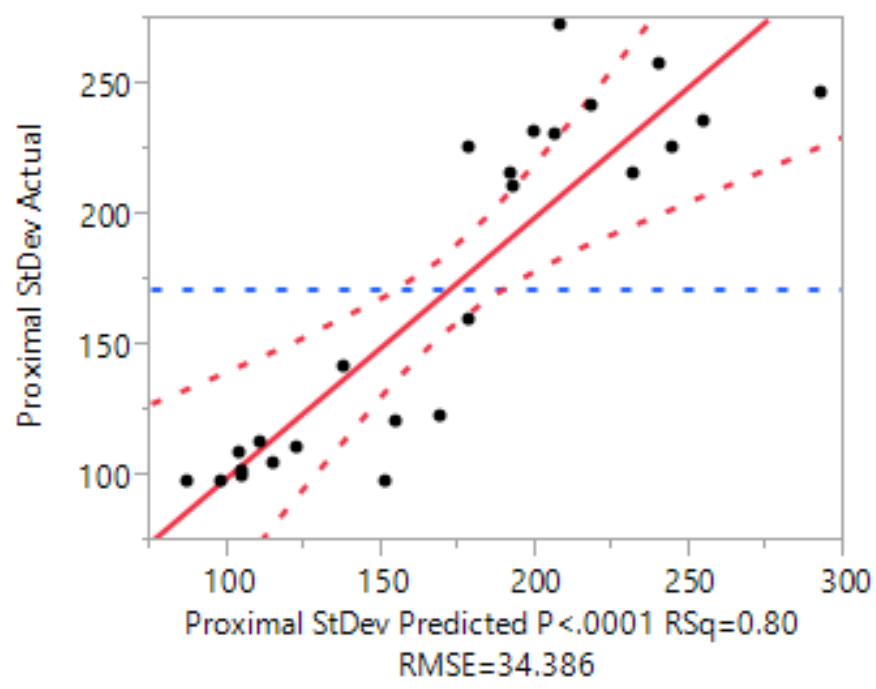

\section{Summary of Fit}

\begin{tabular}{lr}
\hline RSquare & 0.796599 \\
RSquare Adj & 0.712846 \\
Root Mean Square Error & 34.38594 \\
Mean of Response & 171.6974 \\
Observations (or Sum Wgts) & 25
\end{tabular}

\begin{tabular}{|l|r|rrr|}
\hline \multicolumn{5}{l}{ Analysis of Variance } \\
\hline \multicolumn{5}{c|}{ Sum of } \\
Source & DF & Squares & Mean Square & F Ratio \\
Model & 7 & 78722.478 & 11246.1 & 9.5113 \\
\hline Error & 17 & 20100.681 & 1182.4 & Prob > F \\
\hline C. Total & 24 & 98823.159 & & $<.0001^{*}$
\end{tabular}

\section{Parameter Estimates}

$\begin{array}{lrrrrrr}\text { Term } & \text { Estimate } & \text { Std Error } & \text { t Ratio } & \text { Prob }>|\mathbf{t}| & \text { Lower 95\% } & \text { Upper 95\% } \\ \text { Intercept } & 104.41718 & 12.99666 & 8.03 & <.0001^{*} & 76.996614 & 131.83774 \\ \text { A } & -6.184021 & 8.104844 & -0.76 & 0.4559 & -23.28375 & 10.915706 \\ \text { B } & -0.465196 & 8.104844 & -0.06 & 0.9549 & -17.56492 & 16.63453 \\ \text { C } & 19.682267 & 8.104844 & 2.43 & 0.0266^{*} & 2.5825407 & 36.781994 \\ \text { D } & 17.887652 & 8.104844 & 2.21 & 0.0413^{*} & 0.7879251 & 34.987378 \\ B^{\star} \mathrm{C} & 26.931472 & 8.596485 & 3.13 & 0.0061^{*} & 8.7944731 & 45.068471 \\ A^{*} \mathrm{D} & -24.60178 & 8.596485 & -2.86 & 0.0108^{*} & -42.73878 & -6.464784 \\ \mathrm{C}^{\star} \mathrm{C} & 93.444789 & 15.31672 & 6.10 & <.0001^{*} & 61.129343 & 125.76023\end{array}$




\section{Method $2-90^{\text {th }}$ Percentile \% of Intact Stress Model:}

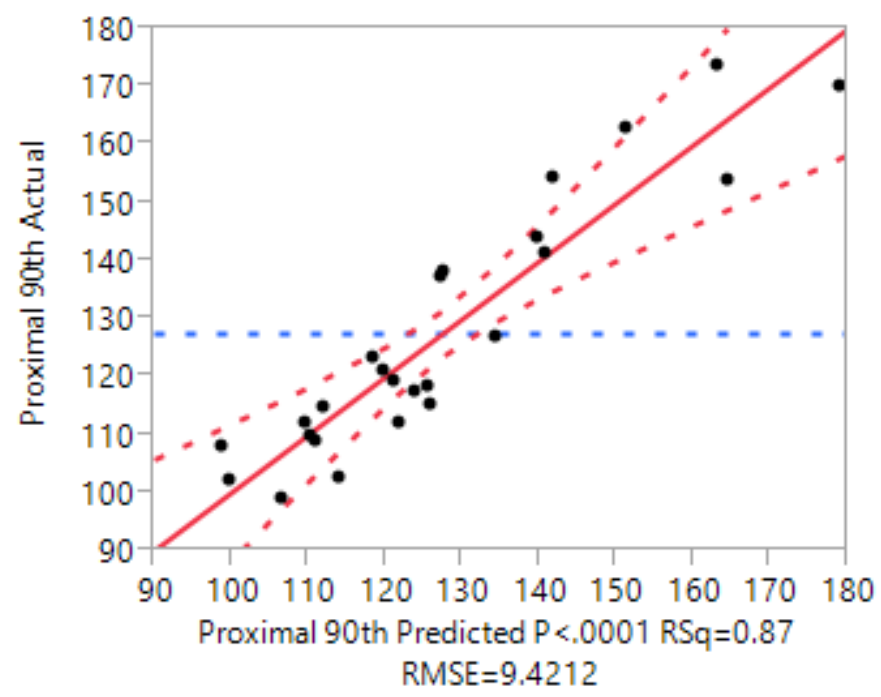

\section{Summary of Fit}

\begin{tabular}{l|r}
\hline RSquare & 0.868494 \\
RSquare Adj & 0.814344 \\
Root Mean Square Error & 9.421224 \\
Mean of Response & 127.459 \\
Observations (or Sum Wgts) & 25
\end{tabular}

\begin{tabular}{l|rrrr|}
\hline \multicolumn{7}{l|}{ Analysis of Variance } \\
\hline \multicolumn{7}{c|}{ Sum of } \\
Source & DF & Squares & Mean Square & F Ratio \\
Model & 7 & 9965.162 & 1423.59 & 16.0388 \\
\hline Error & 17 & 1508.911 & 88.76 & Prob > F \\
C. Total & 24 & 11474.073 & & $<.0001^{*}$
\end{tabular}

\section{Parameter Estimates}

$\begin{array}{lrrrrrr}\text { Term } & \text { Estimate } & \text { Std Error } & \text { t Ratio } & \text { Prob }>|\mathbf{t}| & \text { Lower 95\% } & \text { Upper 95\% } \\ \text { Intercept } & 110.35179 & 3.560888 & 30.99 & <.0001^{*} & 102.83897 & 117.86461 \\ \text { A } & -10.69962 & 2.220604 & -4.82 & 0.0002^{*} & -15.38468 & -6.014554 \\ \text { B } & 0.6684413 & 2.220604 & 0.30 & 0.7671 & -4.016623 & 5.3535059 \\ \text { C } & 6.5917684 & 2.220604 & 2.97 & 0.0086^{*} & 1.9067038 & 11.276833 \\ \text { D } & 11.619217 & 2.220604 & 5.23 & <.0001^{*} & 6.9341525 & 16.304282 \\ B^{\star} \mathrm{C} & 7.288236 & 2.355306 & 3.09 & 0.0066^{*} & 2.3189746 & 12.257497 \\ A^{*} \mathrm{D} & -7.862256 & 2.355306 & -3.34 & 0.0039^{*} & -12.83152 & -2.892995 \\ \mathrm{C}^{\star} \mathrm{C} & 23.760017 & 4.196547 & 5.66 & <.0001^{*} & 14.906077 & 32.613957\end{array}$




\section{Proximal Region \% of Intact Desirability:}

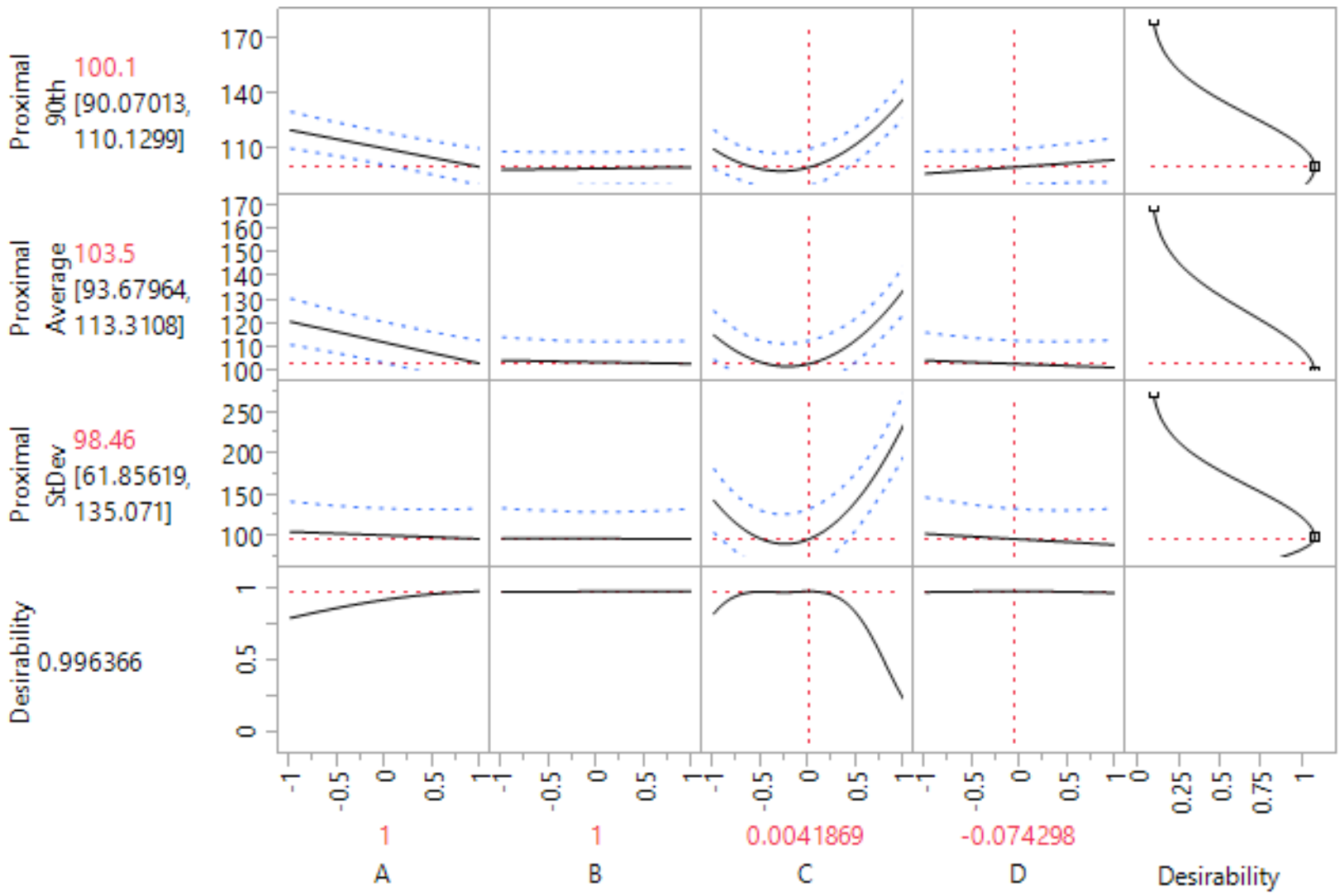




\section{Approach 2 Outcomes - All Regions Mean Stress}

\section{Method 1 - Proximal Region Stress:}

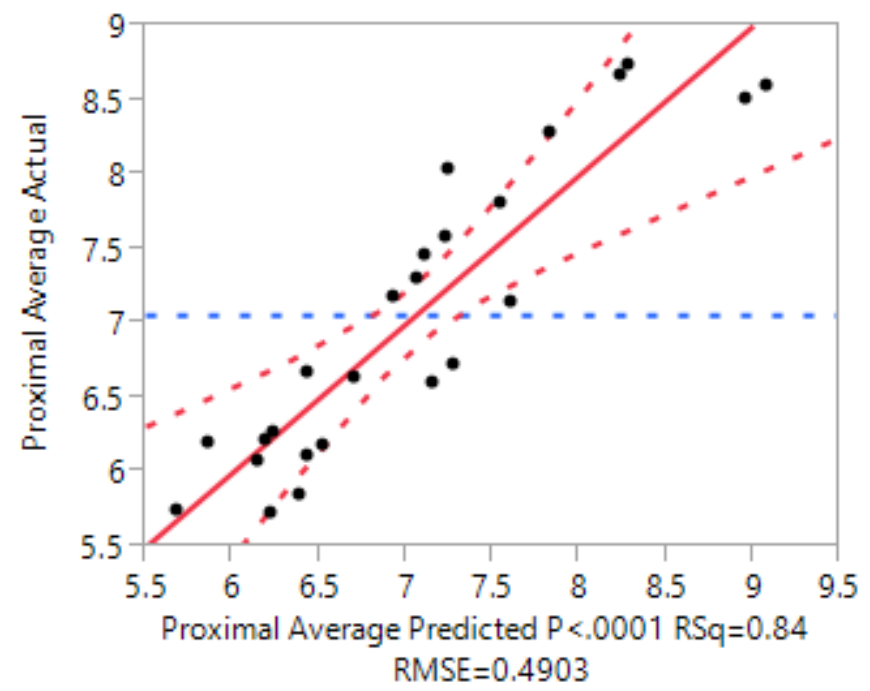

\begin{tabular}{l|r}
\hline Summary of Fit & \\
\hline RSquare & 0.837585 \\
RSquare Adj & 0.756377 \\
Root Mean Square Error & 0.490307 \\
Mean of Response & 7.05376 \\
Observations (or Sum Wgts) & 25
\end{tabular}

\section{Analysis of Variance}

$\begin{array}{lrrrrr} & & \text { Sum of } & & \\ \text { Source } & \text { DF } & \text { Squares } & \text { Mean Square } & \text { F Ratio } \\ \text { Model } & 8 & 19.836215 & 2.47953 & 10.3141 \\ \text { Error } & 16 & 3.846422 & 0.24040 & \text { Prob }>\text { F } \\ \text { C. Total } & 24 & 23.682637 & & <.0001^{*}\end{array}$

\begin{tabular}{lrrrrrrr}
\hline \multicolumn{6}{l}{ Parameter Estimates } \\
\hline Term & Estimate & Std Error & t Ratio & Prob $>|\mathbf{t}|$ & Lower 95\% & Upper 95\% \\
Intercept & 6.1922857 & 0.185319 & 33.41 & $<.0001^{*}$ & 5.7994274 & 6.585144 \\
A & -0.517389 & 0.115567 & -4.48 & $0.0004^{*}$ & -0.762379 & -0.272399 \\
B & -0.041278 & 0.115567 & -0.36 & 0.7256 & -0.286268 & 0.2037124 \\
C & 0.1491111 & 0.115567 & 1.29 & 0.2153 & -0.095879 & 0.3941013 \\
D & 0.3265556 & 0.115567 & 2.83 & $0.0122^{*}$ & 0.0815654 & 0.5715457 \\
$B^{\star}$ C & 0.3806875 & 0.122577 & 3.11 & $0.0068^{*}$ & 0.1208362 & 0.6405388 \\
$A^{*} D$ & -0.409687 & 0.122577 & -3.34 & $0.0041^{*}$ & -0.669539 & -0.149836 \\
$C^{*} D$ & -0.167313 & 0.122577 & -1.36 & 0.1912 & -0.427164 & 0.0925388 \\
$C^{*} \mathrm{C}$ & 1.1964921 & 0.2184 & 5.48 & $<.0001^{*}$ & 0.7335041 & 1.65948
\end{tabular}




\section{Method 1 - Inferior Region Stress:}

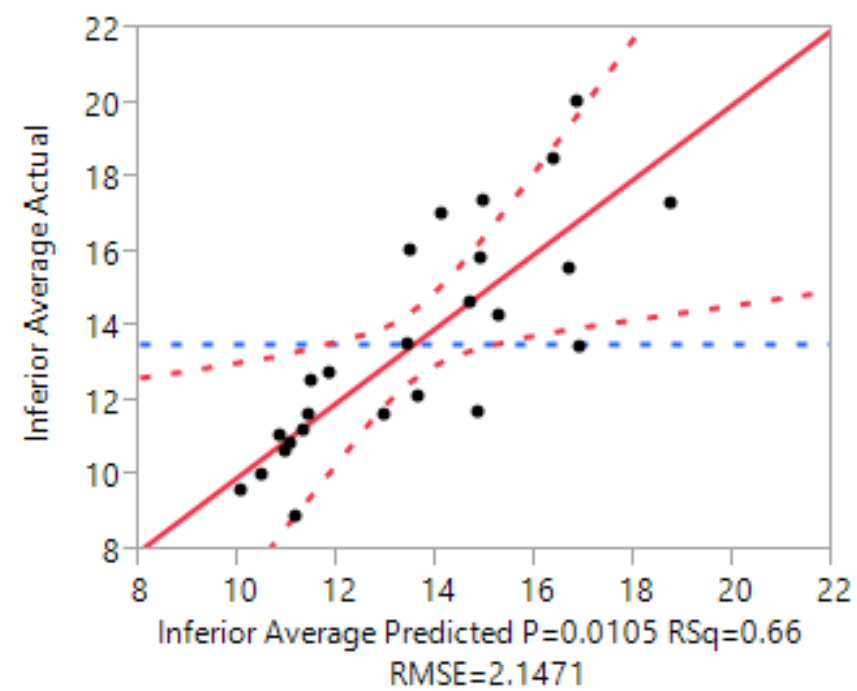

\section{Summary of Fit}

\begin{tabular}{lr}
\hline RSquare & 0.657996 \\
RSquare Adj & 0.486994 \\
Root Mean Square Error & 2.147082 \\
Mean of Response & 13.53512 \\
Observations (or Sum Wgts) & 25
\end{tabular}

\section{Analysis of Variance}

\begin{tabular}{lrrrrr} 
& & Sum of & & \\
Source & DF & Squares & Mean Square & F Ratio \\
\hline Model & 8 & 141.90865 & 17.7386 & 3.8479 \\
\hline Error & 16 & 73.75938 & 4.6100 & Prob > F \\
C. Total & 24 & 215.66803 & & $0.0105^{*}$
\end{tabular}

\section{Parameter Estimates}

\begin{tabular}{lrrrrrr} 
Term & Estimate & Std Error & t Ratio & Prob $>|\mathbf{t}|$ & Lower 95\% & Upper 95\% \\
\hline Intercept & 10.944286 & 0.811521 & 13.49 & $<.0001^{*}$ & 9.2239386 & 12.664633 \\
A & -0.898778 & 0.506072 & -1.78 & 0.0948 & -1.971603 & 0.1740471 \\
\hline B & -0.086833 & 0.506072 & -0.17 & 0.8659 & -1.159658 & 0.9859916 \\
C & 0.4237778 & 0.506072 & 0.84 & 0.4147 & -0.649047 & 1.4966027 \\
D & 0.4621111 & 0.506072 & 0.91 & 0.3747 & -0.610714 & 1.534936 \\
$B^{\star} \mathrm{C}$ & 1.082625 & 0.536771 & 2.02 & 0.0608 & -0.055278 & 2.2205276 \\
$A^{\star} D$ & -0.823 & 0.536771 & -1.53 & 0.1447 & -1.960903 & 0.3149026 \\
$C^{\star} D$ & -1.257625 & 0.536771 & -2.34 & $0.0324^{*}$ & -2.395528 & -0.119722 \\
$C^{\star} \mathrm{C}$ & 3.598381 & 0.956386 & 3.76 & $0.0017^{\star}$ & 1.5709325 & 5.6258295
\end{tabular}




\section{Method 1 - Superior Region Stress:}

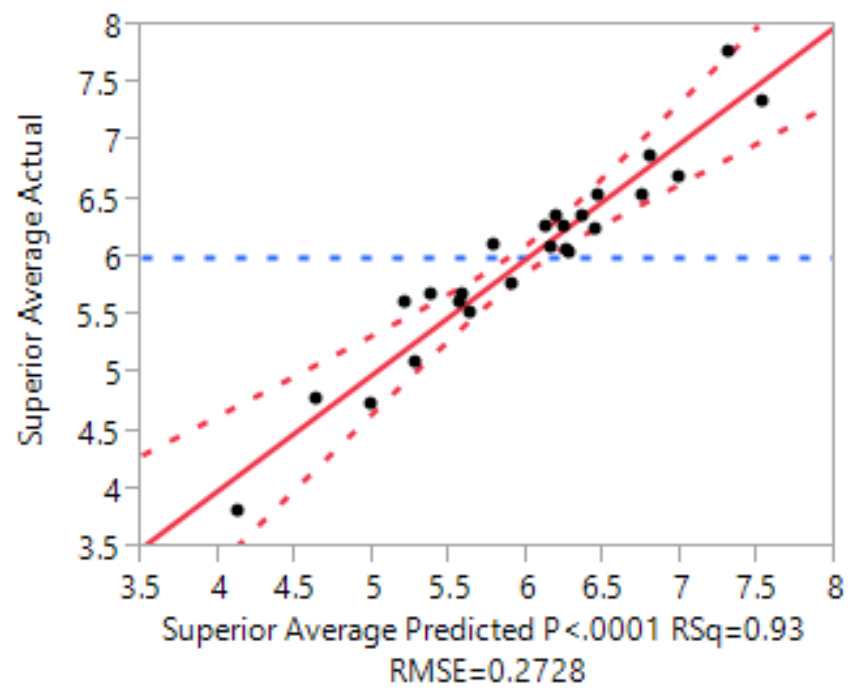

\section{Summary of Fit}

\begin{tabular}{l|r}
\hline RSquare & 0.928664 \\
\hline RSquare Adj & 0.892995 \\
Root Mean Square Error & 0.272804 \\
Mean of Response & 5.9978 \\
Observations (or Sum Wgts) & 25
\end{tabular}

\begin{tabular}{|c|c|c|c|c|}
\hline \multicolumn{5}{|c|}{ Analysis of Variance } \\
\hline Source & DF & $\begin{array}{r}\text { Sum of } \\
\text { Squares }\end{array}$ & Mean Square & F Ratio \\
\hline Model & 8 & 15.501319 & 1.93766 & 26.0362 \\
\hline Error & 16 & 1.190753 & 0.07442 & Prob $>F$ \\
\hline C. Total & 24 & 16.692072 & & $<.0001^{*}$ \\
\hline
\end{tabular}

\section{Parameter Estimates}

$\begin{array}{lrrrrrr}\text { Term } & \text { Estimate } & \text { Std Error } & \text { t Ratio } & \text { Prob }>|\mathbf{t}| & \text { Lower 95\% } & \text { Upper 95\% } \\ \text { Intercept } & 6.1897143 & 0.10311 & 60.03 & <.0001^{*} & 5.9711303 & 6.4082983 \\ \text { A } & -0.559722 & 0.064301 & -8.70 & <.0001^{*} & -0.696033 & -0.423411 \\ \text { B } & 0.0751111 & 0.064301 & 1.17 & 0.2599 & -0.0612 & 0.2114222 \\ \text { C } & -0.354056 & 0.064301 & -5.51 & <.0001^{*} & -0.490367 & -0.217745 \\ \text { D } & -0.606333 & 0.064301 & -9.43 & <.0001^{*} & -0.742644 & -0.470022 \\ B^{\star} \mathrm{C} & 0.1805625 & 0.068201 & 2.65 & 0.0176^{*} & 0.0359828 & 0.3251422 \\ A^{\star} D & -0.019938 & 0.068201 & -0.29 & 0.7738 & -0.164517 & 0.1246422 \\ C^{\star} D & -0.005938 & 0.068201 & -0.09 & 0.9317 & -0.150517 & 0.1386422 \\ C^{\star} \mathrm{C} & -0.266548 & 0.121517 & -2.19 & 0.0434^{*} & -0.524151 & -0.008944\end{array}$




\section{Method 1 - Lateral Region Stress:}

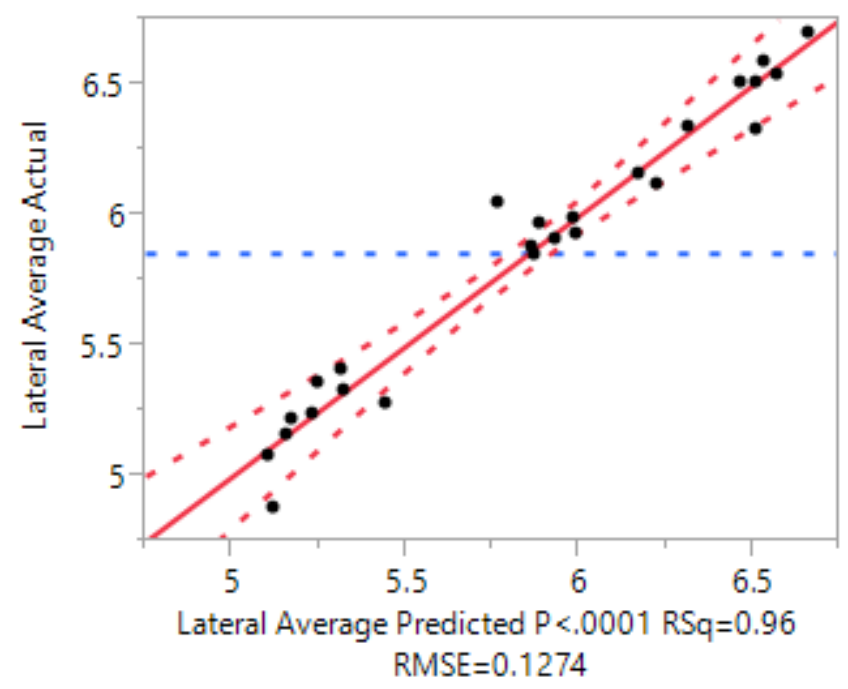

\section{Summary of Fit}

\begin{tabular}{lr}
\hline RSquare & 0.96375 \\
RSquare Adj & 0.945625 \\
Root Mean Square Error & 0.127358 \\
Mean of Response & 5.85376 \\
Observations (or Sum Wgts) & 25
\end{tabular}

\begin{tabular}{lrrrrr|}
\hline \multicolumn{5}{|c|}{ Analysis of Variance } \\
\\
Source & DF & $\begin{array}{r}\text { Sum of } \\
\text { Squares }\end{array}$ & Mean Square & F Ratio \\
Model & 8 & 6.8996547 & 0.862457 & 53.1721 \\
Error & 16 & 0.2595218 & 0.016220 & Prob > F \\
C. Total & 24 & 7.1591766 & & $<.0001^{*}$
\end{tabular}

\section{Parameter Estimates}

$\begin{array}{lrrrrrrr}\text { Term } & \text { Estimate } & \text { Std Error } & \text { t Ratio } & \text { Prob }>|\mathbf{t}| & \text { Lower 95\% } & \text { Upper 95\% } \\ \text { Intercept } & 5.927 & 0.048137 & 123.13 & <.0001^{*} & 5.8249544 & 6.0290456 \\ \text { A } & -0.602222 & 0.030019 & -20.06 & <.0001^{*} & -0.665859 & -0.538586 \\ \text { B } & 0.0628889 & 0.030019 & 2.09 & 0.0524 & -0.000748 & 0.1265255 \\ \text { C } & 0.0588333 & 0.030019 & 1.96 & 0.0677 & -0.004803 & 0.12247 \\ \text { D } & 0.054 & 0.030019 & 1.80 & 0.0909 & -0.009637 & 0.1176366 \\ B^{\star} \mathrm{C} & 0.0351875 & 0.03184 & 1.11 & 0.2854 & -0.032309 & 0.1026843 \\ A^{\star} D & -0.068062 & 0.03184 & -2.14 & 0.0483^{*} & -0.135559 & -0.000566 \\ C^{\star} D & -0.049688 & 0.03184 & -1.56 & 0.1382 & -0.117184 & 0.0178093 \\ C^{\star} \mathrm{C} & -0.101722 & 0.05673 & -1.79 & 0.0919 & -0.221984 & 0.0185397\end{array}$




\section{All Regions Mean Stress Desirability:}

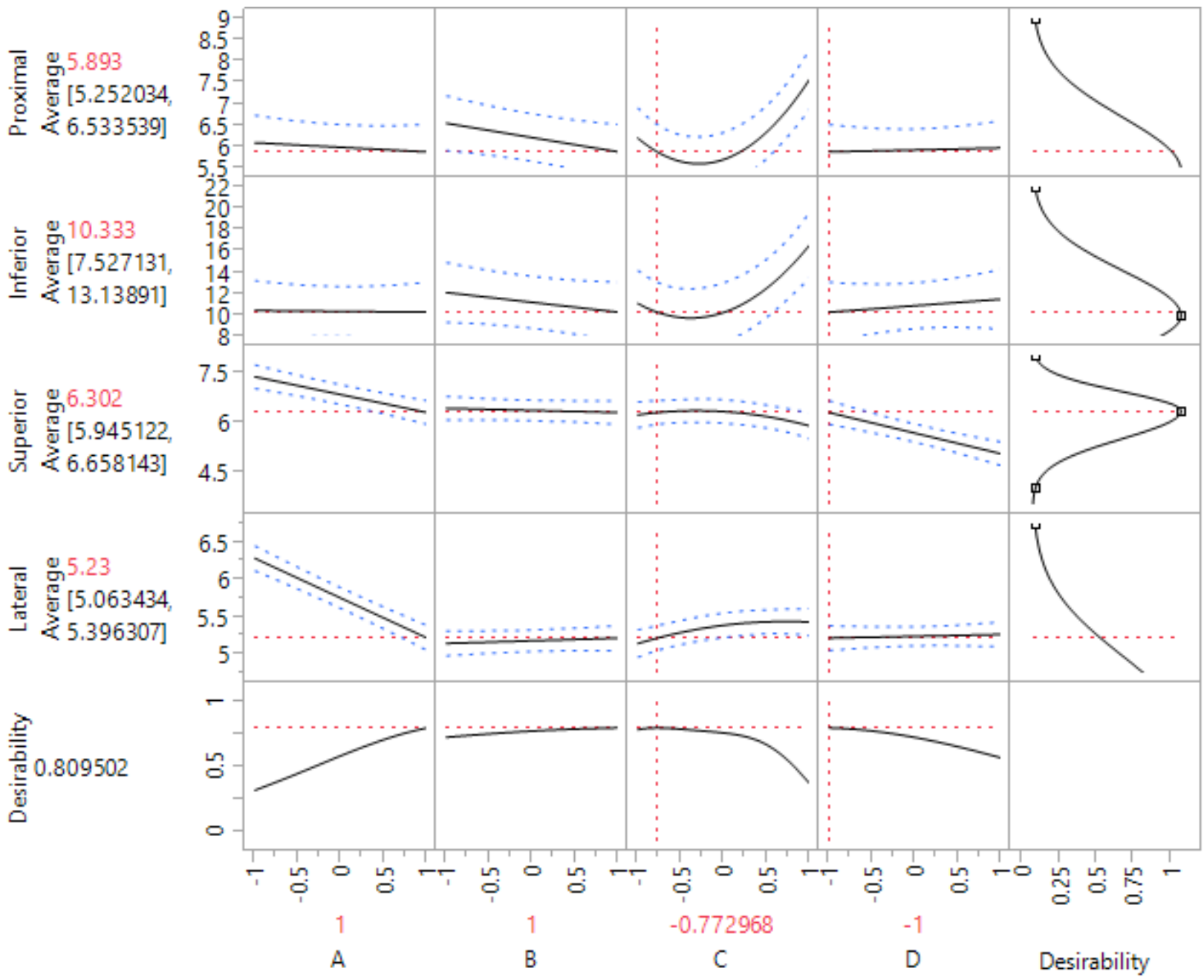


Method 2 - Proximal Region \% of Intact:

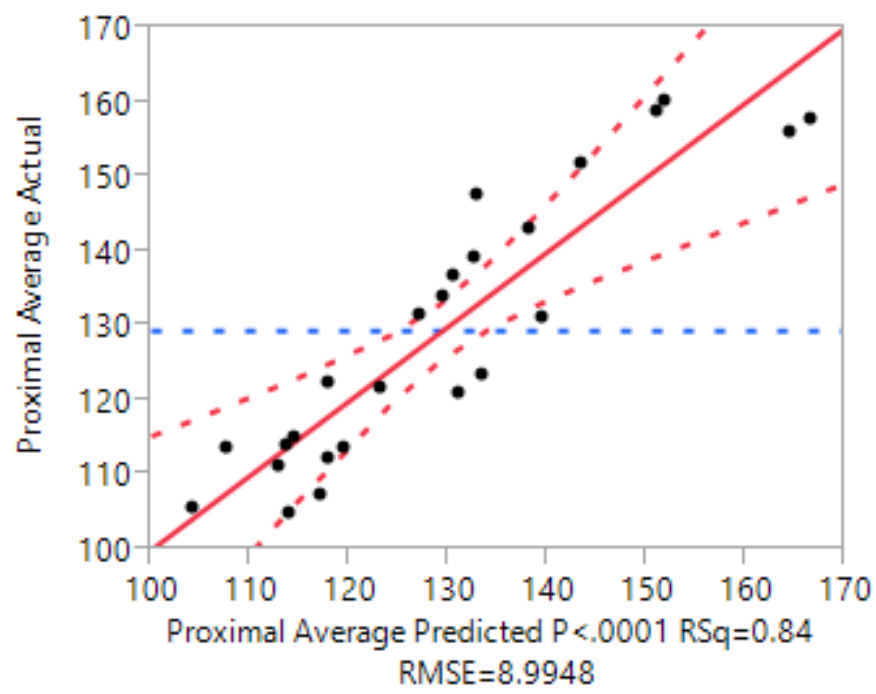

Summary of Fit

$\begin{array}{lr}\text { RSquare } & 0.837529 \\ \text { RSquare Adj } & 0.756294 \\ \text { Root Mean Square Error } & 8.994829 \\ \text { Mean of Response } & 129.3869 \\ \text { Observations (or Sum Wgts) } & 25\end{array}$

Analysis of Variance

\begin{tabular}{|c|c|c|c|c|}
\hline Source & DF & $\begin{array}{r}\text { Sum of } \\
\text { Squares }\end{array}$ & Mean Square & F Ratio \\
\hline Model & 8 & 6673.1363 & 834.142 & 10.3099 \\
\hline Error & 16 & 1294.5112 & 80.907 & Prob $>F$ \\
\hline C. Total & 24 & 7967.6475 & & $<.0001^{*}$ \\
\hline
\end{tabular}

\section{Parameter Estimates}

\begin{tabular}{lrrrrrr}
\hline Term & Estimate & Std Error & t Ratio & Prob $>|\mathbf{t}|$ & Lower 95\% & Upper 95\% \\
Intercept & 113.58719 & 3.399726 & 33.41 & $<.0001^{*}$ & 106.38009 & 120.79429 \\
A & -9.488682 & 2.120102 & -4.48 & $0.0004^{*}$ & -13.9831 & -4.994267 \\
B & -0.757854 & 2.120102 & -0.36 & 0.7254 & -5.252269 & 3.7365602 \\
C & 2.7334636 & 2.120102 & 1.29 & 0.2156 & -1.760951 & 7.227878 \\
D & 5.9894365 & 2.120102 & 2.83 & $0.0122^{*}$ & 1.4950221 & 10.483851 \\
$B^{\star} \mathrm{C}$ & 6.9829582 & 2.248707 & 3.11 & $0.0068^{*}$ & 2.2159118 & 11.750005 \\
$A^{\star} \mathrm{D}$ & -7.517357 & 2.248707 & -3.34 & $0.0041^{*}$ & -12.2844 & -2.750311 \\
$\mathrm{C}^{\star} \mathrm{D}$ & -3.068151 & 2.248707 & -1.36 & 0.1913 & -7.835197 & 1.6988957 \\
$\mathrm{C}^{\star} \mathrm{C}$ & 21.944098 & 4.006615 & 5.48 & $<.0001^{*}$ & 13.450453 & 30.437743
\end{tabular}




\section{Method 2 - Inferior Region \% of Intact:}

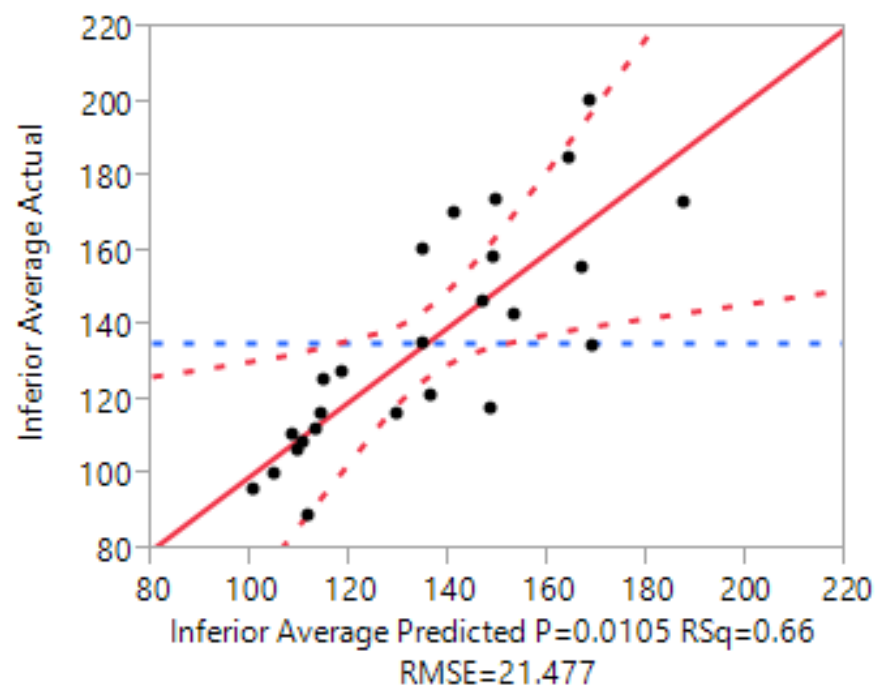

\section{Summary of Fit}

\begin{tabular}{lr}
\hline RSquare & 0.658007 \\
RSquare Adj & 0.48701 \\
Root Mean Square Error & 21.47684 \\
Mean of Response & 135.3918 \\
Observations (or Sum Wgts) & 25
\end{tabular}

\begin{tabular}{lrrrrr|}
\hline \multicolumn{5}{l}{ Analysis of Variance } \\
Source & DF & Sum of & & \\
Squares & Mean Square & F Ratio \\
Model & 8 & 14199.525 & 1774.94 & 3.8481 \\
Error & 16 & 7380.072 & 461.25 & Prob > F \\
C. Total & 24 & 21579.597 & & $0.0105^{*}$
\end{tabular}

\section{Parameter Estimates}

$\begin{array}{lrrrrrr}\text { Term } & \text { Estimate } & \text { Std Error } & \text { t Ratio } & \text { Prob }>|\mathbf{t}| & \text { Lower 95\% } & \text { Upper 95\% } \\ \text { Intercept } & 109.47553 & 8.117481 & 13.49 & <.0001^{*} & 92.267239 & 126.68382 \\ \text { A } & -8.990583 & 5.062139 & -1.78 & 0.0947 & -19.72184 & 1.7406717 \\ \text { B } & -0.868092 & 5.062139 & -0.17 & 0.8660 & -11.59935 & 9.8631632 \\ \text { C } & 4.239716 & 5.062139 & 0.84 & 0.4146 & -6.491539 & 14.970971 \\ \text { D } & 4.6233002 & 5.062139 & 0.91 & 0.3746 & -6.107955 & 15.354555 \\ B^{\star} \mathrm{C} & 10.829175 & 5.369209 & 2.02 & 0.0608 & -0.55304 & 22.21139 \\ A^{*} D & -8.232864 & 5.369209 & -1.53 & 0.1447 & -19.61508 & 3.1493505 \\ C^{\star} D & -12.57956 & 5.369209 & -2.34 & 0.0324^{\star} & -23.96177 & -1.197341 \\ C^{\star} \mathrm{C} & 35.994838 & 9.566543 & 3.76 & 0.0017^{\star} & 15.714672 & 56.275004\end{array}$




\section{Method 2 - Superior Region \% of Intact:}

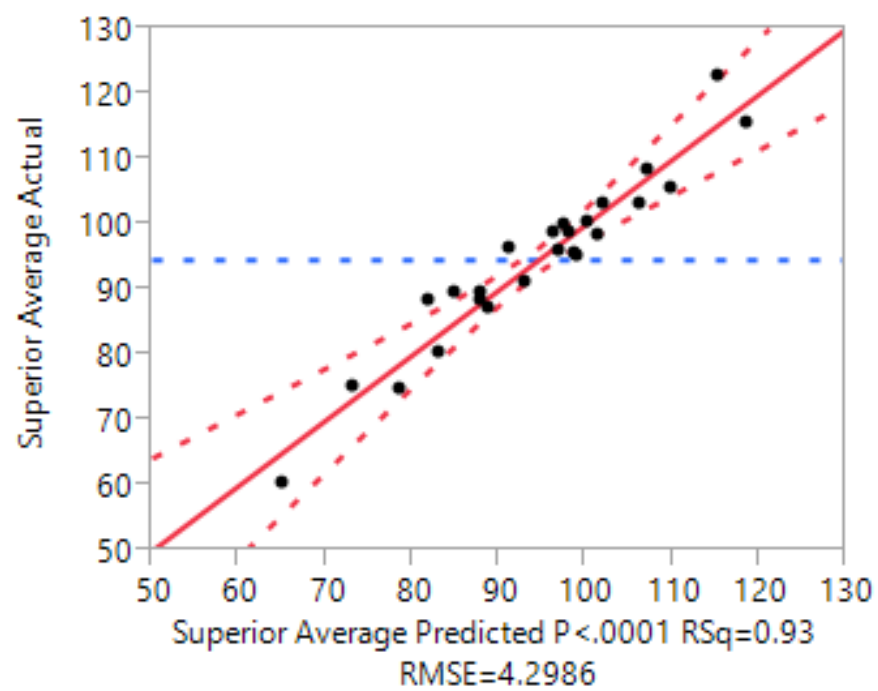

\section{Summary of Fit}

\begin{tabular}{lr}
\hline RSquare & 0.928681 \\
RSquare Adj & 0.893022 \\
Root Mean Square Error & 4.298623 \\
Mean of Response & 94.52402 \\
Observations (or Sum Wgts) & 25
\end{tabular}

\begin{tabular}{|c|c|c|c|c|}
\hline \multicolumn{5}{|c|}{ Analysis of Variance } \\
\hline Source & DF & $\begin{array}{l}\text { Sum of } \\
\text { Squares }\end{array}$ & Mean Square & F Ratio \\
\hline Model & 8 & 3849.8264 & 481.228 & 26.0431 \\
\hline Error & 16 & 295.6505 & 18.478 & Prob $>F$ \\
\hline C. Total & 24 & 4145.4769 & & $<.0001 *$ \\
\hline
\end{tabular}

\section{Parameter Estimates}

$\begin{array}{lrrrrrr}\text { Term } & \text { Estimate } & \text { Std Error } & \text { t Ratio } & \text { Prob }>|\mathbf{t}| & \text { Lower 95\% } & \text { Upper 95\% } \\ \text { Intercept } & 97.549312 & 1.624727 & 60.04 & <.0001^{*} & 94.105045 & 100.99358 \\ \text { A } & -8.820771 & 1.013195 & -8.71 & <.0001^{*} & -10.96865 & -6.672893 \\ \text { B } & 1.1824112 & 1.013195 & 1.17 & 0.2603 & -0.965466 & 3.3302888 \\ \text { C } & -5.579482 & 1.013195 & -5.51 & <.0001^{*} & -7.727359 & -3.431604 \\ \text { D } & -9.555477 & 1.013195 & -9.43 & <.0001^{*} & -11.70335 & -7.4076 \\ B^{\star} \mathrm{C} & 2.8458994 & 1.074656 & 2.65 & 0.0175^{*} & 0.5677312 & 5.1240676 \\ A^{\star} D & -0.312467 & 1.074656 & -0.29 & 0.7750 & -2.590635 & 1.9657012 \\ C^{\star} \mathrm{D} & -0.094539 & 1.074656 & -0.09 & 0.9310 & -2.372707 & 2.1836294 \\ C^{\star} \mathrm{C} & -4.201792 & 1.914759 & -2.19 & 0.0433^{*} & -8.2609 & -0.142685\end{array}$




\section{Method 2 - Lateral Region \% of Intact:}

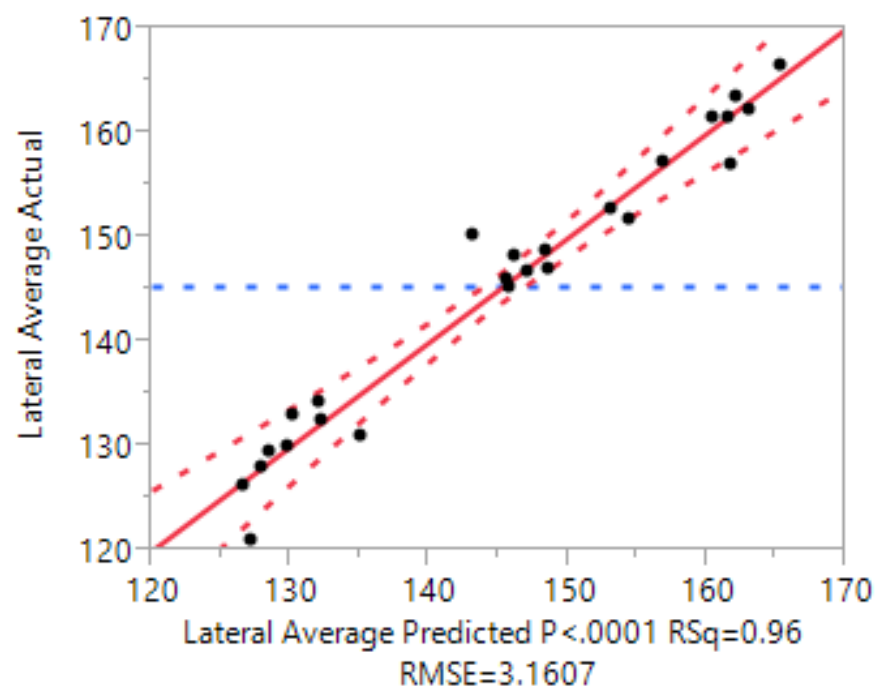

\section{Summary of Fit}

\begin{tabular}{|c|c|c|c|c|}
\hline \multicolumn{3}{|c|}{ RSquare } & \multicolumn{2}{|l|}{0.963747} \\
\hline \multicolumn{3}{|c|}{ RSquare Adj } & 0.94562 & \\
\hline \multicolumn{3}{|c|}{ Root Mean Square Error } & 3.160714 & \\
\hline \multicolumn{3}{|c|}{ Mean of Response } & 145.2828 & \\
\hline \multicolumn{3}{|c|}{ Observations (or Sum Wgts) } & 25 & \\
\hline \multicolumn{5}{|c|}{ Analysis of Variance } \\
\hline Source & DF & $\begin{array}{r}\text { Sum of } \\
\text { Squares }\end{array}$ & Mean Square & F Ratio \\
\hline Model & 8 & 4249.2133 & 531.152 & 53.1677 \\
\hline Error & 16 & 159.8419 & 9.990 & Prob $>F$ \\
\hline C. Total & 24 & 4409.0551 & & $<.0001^{*}$ \\
\hline
\end{tabular}

\section{Parameter Estimates}

$\begin{array}{lrrrrrr}\text { Term } & \text { Estimate } & \text { Std Error } & \text { t Ratio } & \text { Prob }>|\mathbf{t}| & \text { Lower 95\% } & \text { Upper 95\% } \\ \text { Intercept } & 147.09972 & 1.194638 & 123.13 & <.0001^{*} & 144.5672 & 149.63224 \\ \text { A } & -14.94444 & 0.744988 & -20.06 & <.0001^{*} & -16.52374 & -13.36513 \\ \text { B } & 1.5620332 & 0.744988 & 2.10 & 0.0523 & -0.01727 & 3.1413363 \\ \text { C } & 1.4612548 & 0.744988 & 1.96 & 0.0675 & -0.118048 & 3.0405578 \\ \text { D } & 1.3431307 & 0.744988 & 1.80 & 0.0903 & -0.236172 & 2.9224338 \\ B^{\star} \mathrm{C} & 0.8717527 & 0.790179 & 1.10 & 0.2862 & -0.803351 & 2.5468566 \\ A^{\star} D & -1.69032 & 0.790179 & -2.14 & 0.0482^{*} & -3.365424 & -0.015216 \\ C^{\star} D & -1.234099 & 0.790179 & -1.56 & 0.1379 & -2.909203 & 0.4410051 \\ C^{\star} \mathrm{C} & -2.523473 & 1.407894 & -1.79 & 0.0920 & -5.508076 & 0.4611289\end{array}$




\section{All Regions \% of Intact Desirability:}

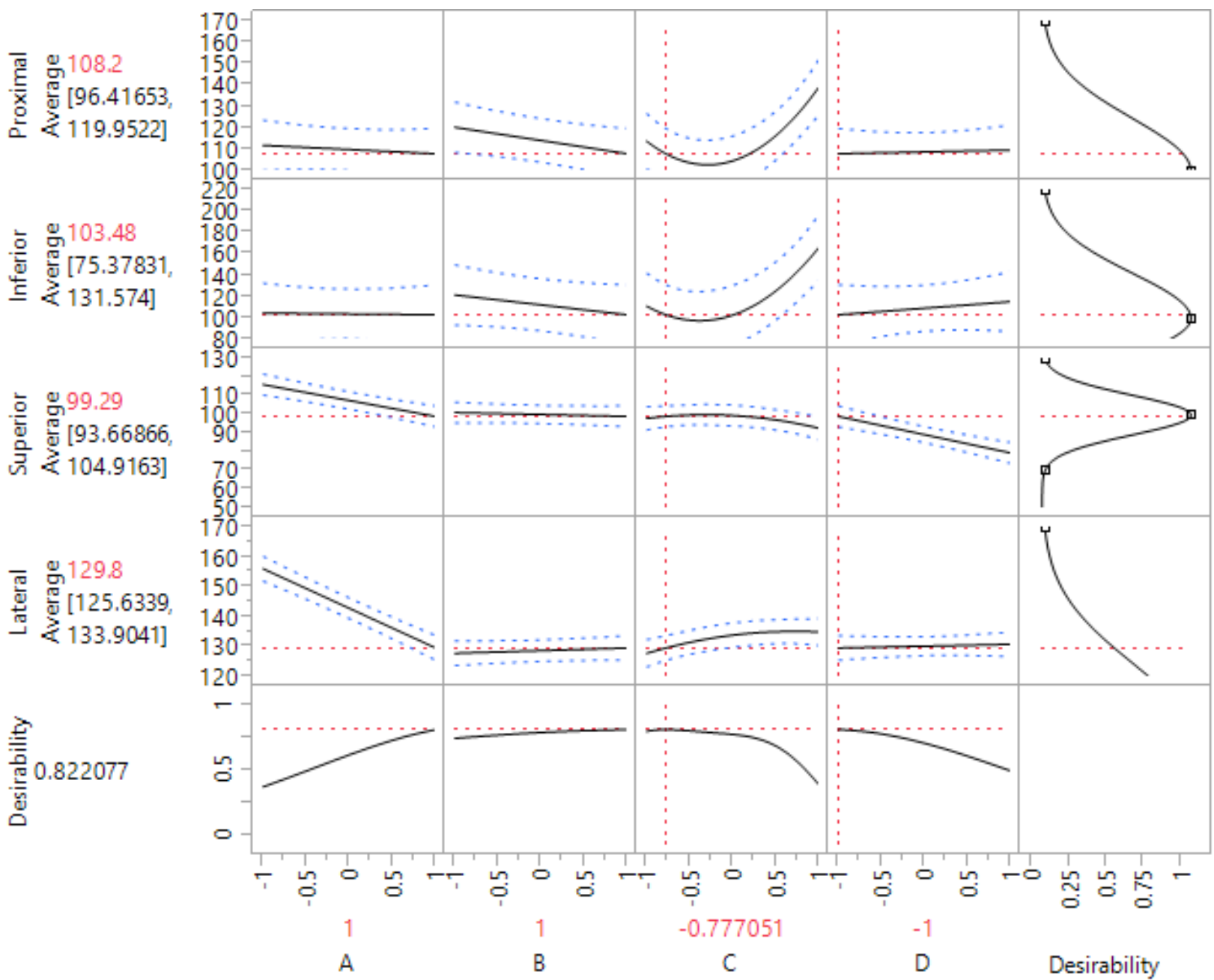




\section{Approach 3 Outcomes - All Regions $90^{\text {th }}$ Percentile}

\section{Method 1 - Proximal Region Stress:}

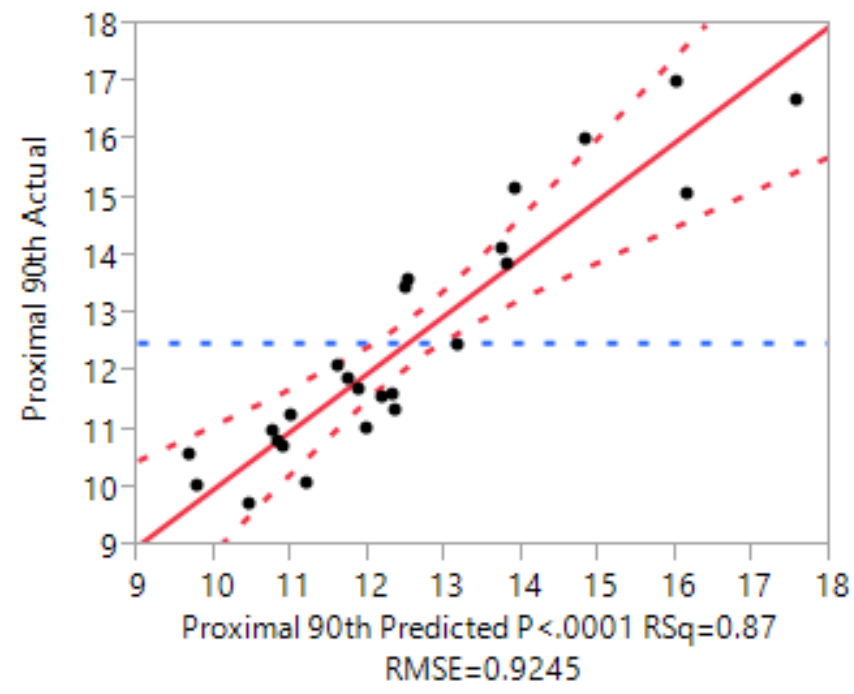

\begin{tabular}{l|r|}
\hline Summary of Fit & \\
\hline RSquare & 0.868507 \\
RSquare Adj & 0.814362 \\
Root Mean Square Error & 0.924466 \\
Mean of Response & 12.50812 \\
\hline Observations (or Sum Wgts) & 25 \\
\hline
\end{tabular}

Analysis of Variance

\begin{tabular}{|c|c|c|c|c|}
\hline Source & DF & $\begin{array}{l}\text { Sum of } \\
\text { Squares }\end{array}$ & Mean Square & F Ratio \\
\hline Model & 7 & 95.96203 & 13.7089 & 16.0406 \\
\hline Error & 17 & 14.52883 & 0.8546 & Prob $>F$ \\
\hline Total & 24 & 110.49085 & & $<.0001^{*}$ \\
\hline
\end{tabular}

\section{Parameter Estimates}

$\begin{array}{lrrrrrr}\text { Term } & \text { Estimate } & \text { Std Error } & \text { t Ratio } & \text { Prob }>|\mathbf{t}| & \text { Lower 95\% } & \text { Upper 95\% } \\ \text { Intercept } & 10.829286 & 0.349415 & 30.99 & <.0001^{*} & 10.092084 & 11.566487 \\ \text { A } & -1.05 & 0.217899 & -4.82 & 0.0002^{*} & -1.509726 & -0.590274 \\ \text { B } & 0.0655 & 0.217899 & 0.30 & 0.7674 & -0.394226 & 0.525226 \\ \text { C } & 0.6468889 & 0.217899 & 2.97 & 0.0086^{*} & 0.1871629 & 1.1066149 \\ \text { D } & 1.1401667 & 0.217899 & 5.23 & <.0001^{*} & 0.6804407 & 1.5998926 \\ \mathrm{~B}^{\star} \mathrm{C} & 0.715125 & 0.231116 & 3.09 & 0.0066^{*} & 0.227512 & 1.202738 \\ \mathrm{~A}^{*} \mathrm{D} & -0.7715 & 0.231116 & -3.34 & 0.0039^{*} & -1.259113 & -0.283887 \\ \mathrm{C}^{\star} \mathrm{C} & 2.3317143 & 0.41179 & 5.66 & <.0001^{*} & 1.4629139 & 3.2005147\end{array}$




\section{Method 1 - Inferior Region Stress:}

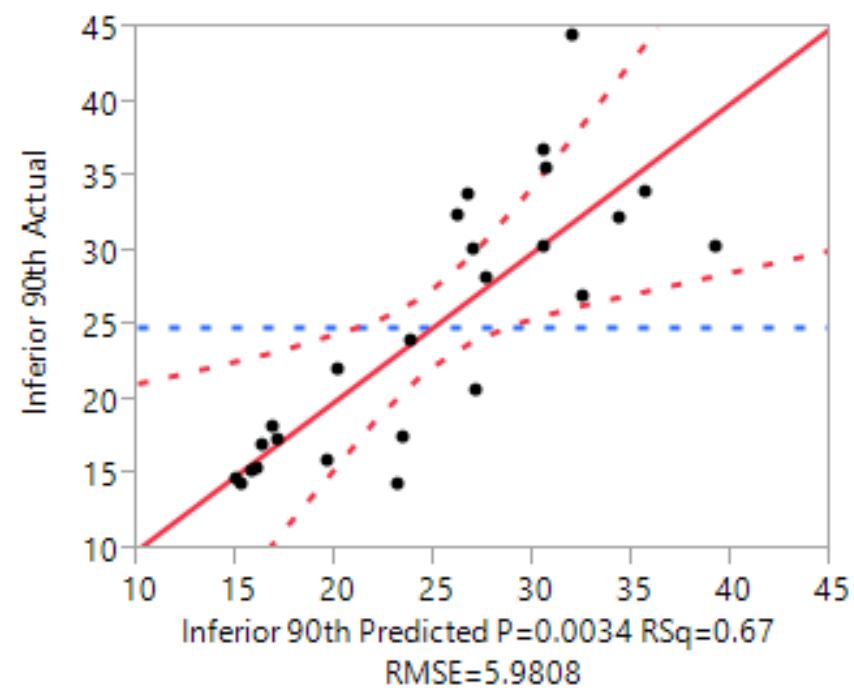

\section{Summary of Fit}

\begin{tabular}{l|r}
\hline RSquare & 0.670701 \\
RSquare Adj & 0.535108 \\
Root Mean Square Error & 5.980775 \\
Mean of Response & 24.90104 \\
Observations (or Sum Wgts) & 25
\end{tabular}

Analysis of Variance

\begin{tabular}{|c|c|c|c|c|}
\hline Source & DF & $\begin{array}{r}\text { Sum of } \\
\text { Squares }\end{array}$ & Mean Square & F Ratio \\
\hline Model & 7 & 1238.5191 & 176.931 & 4.9464 \\
\hline Error & 17 & 608.0844 & 35.770 & Prob $>F$ \\
\hline C. Total & 24 & 1846.6035 & & $0.0034^{*}$ \\
\hline
\end{tabular}

\section{Parameter Estimates}

$\begin{array}{lrrrrrr}\text { Term } & \text { Estimate } & \text { Std Error } & \text { t Ratio } & \text { Prob }>|\mathbf{t}| & \text { Lower 95\% } & \text { Upper 95\% } \\ \text { Intercept } & 16.074143 & 2.26052 & 7.11 & <.0001^{*} & 11.304862 & 20.843424 \\ \text { A } & -0.765944 & 1.409682 & -0.54 & 0.5939 & -3.740114 & 2.208225 \\ \text { B } & 0.2767778 & 1.409682 & 0.20 & 0.8467 & -2.697392 & 3.2509472 \\ \text { C } & 2.1656111 & 1.409682 & 1.54 & 0.1429 & -0.808558 & 5.1397806 \\ \text { D } & 1.0191667 & 1.409682 & 0.72 & 0.4795 & -1.955003 & 3.9933361 \\ B^{\star} \mathrm{C} & 4.0309375 & 1.495194 & 2.70 & 0.0153^{*} & 0.8763544 & 7.1855206 \\ \text { A D }^{*} & -2.573813 & 1.495194 & -1.72 & 0.1033 & -5.728396 & 0.5807706 \\ \text { C }^{*} \mathrm{C} & 12.259579 & 2.664049 & 4.60 & 0.0003^{*} & 6.6389274 & 17.880231\end{array}$




\section{Method 1 - Superior Region Stress:}

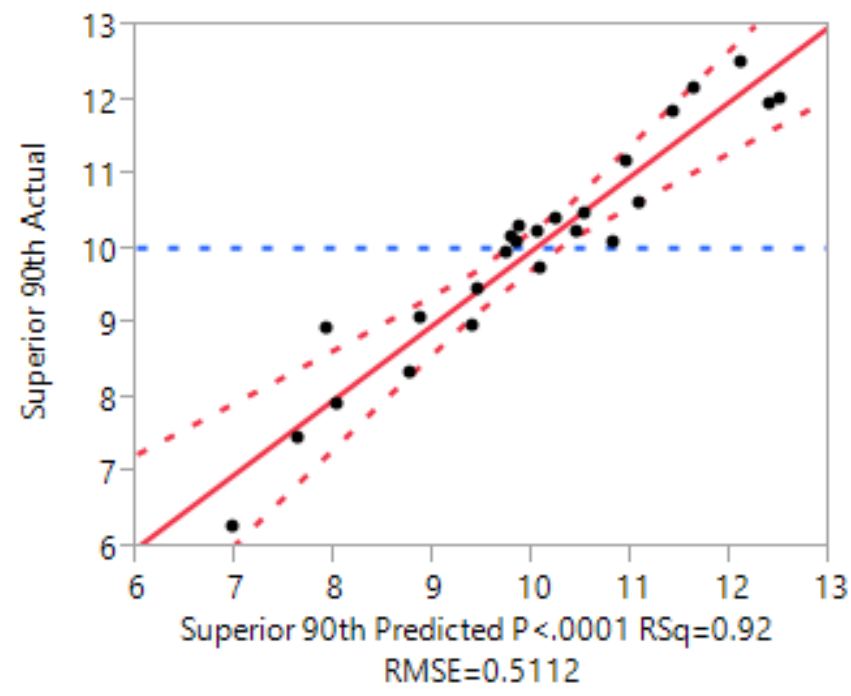

\section{Summary of Fit}

$\begin{array}{lr}\text { RSquare } & 0.919732 \\ \text { RSquare Adj } & 0.886681 \\ \text { Root Mean Square Error } & 0.51122 \\ \text { Mean of Response } & 10.02124 \\ \text { Observations (or Sum Wgts) } & 25\end{array}$

\section{Analysis of Variance}

$\begin{array}{lrrrrr} & & \text { Sum of } & & \\ \text { Source } & \text { DF } & \text { Squares } & \text { Mean Square } & \text { F Ratio } \\ \text { Model } & 7 & 50.907988 & 7.27257 & 27.8274 \\ \text { Error } & 17 & 4.442877 & 0.26135 & \text { Prob }>\text { F } \\ \text { C. Total } & 24 & 55.350865 & & <.0001^{*}\end{array}$

\section{Parameter Estimates}

\begin{tabular}{lrrrrrr} 
Term & Estimate & Std Error & t Ratio & Prob $>|\mathbf{t}|$ & Lower 95\% & Upper 95\% \\
\hline Intercept & 10.246857 & 0.193223 & 53.03 & $<.0001^{*}$ & 9.8391924 & 10.654522 \\
\hline A & -0.841944 & 0.120496 & -6.99 & $<.0001^{*}$ & -1.096168 & -0.587721 \\
B & 0.1925556 & 0.120496 & 1.60 & 0.1285 & -0.061668 & 0.4467792 \\
C & -0.1385 & 0.120496 & -1.15 & 0.2663 & -0.392724 & 0.1157236 \\
D & -1.388833 & 0.120496 & -11.53 & $<.0001^{*}$ & -1.643057 & -1.13461 \\
B $^{\star}$ C & 0.34125 & 0.127805 & 2.67 & $0.0162^{*}$ & 0.0716051 & 0.6108949 \\
A $^{\star}$ D & -0.06025 & 0.127805 & -0.47 & 0.6433 & -0.329895 & 0.2093949 \\
C $^{\star} \mathrm{C}$ & -0.313357 & 0.227715 & -1.38 & 0.1867 & -0.793795 & 0.1670804
\end{tabular}




\section{Method 1 - Lateral Region Stress:}

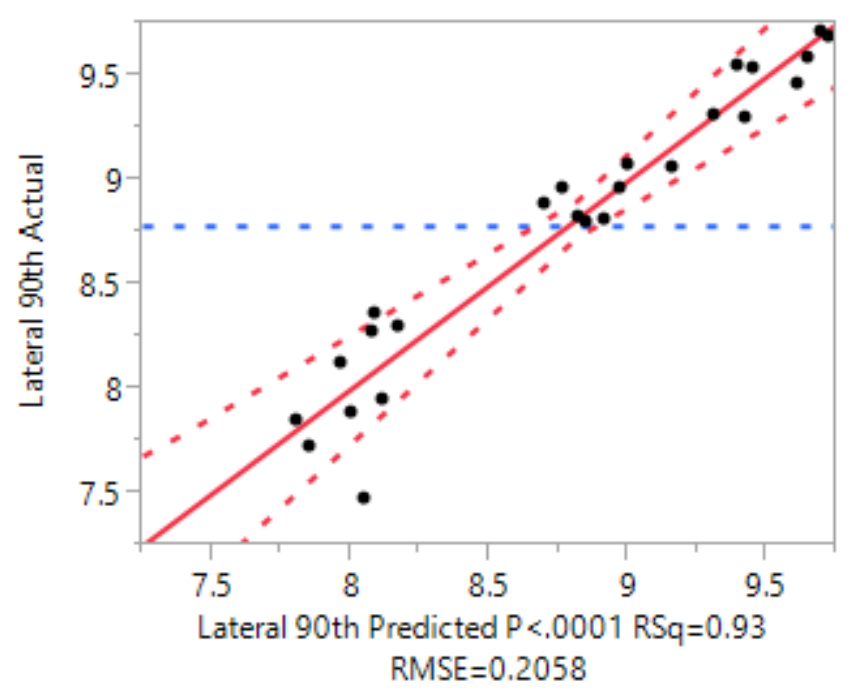

\section{Summary of Fit}

\begin{tabular}{l|r}
\hline RSquare & 0.934863 \\
RSquare Adj & 0.908041 \\
Root Mean Square Error & 0.205765 \\
Mean of Response & 8.7804 \\
Observations (or Sum Wgts) & 25
\end{tabular}

\section{Analysis of Variance}

\begin{tabular}{lrrrrc} 
& \multicolumn{5}{c}{ Sum of } \\
Source & DF & Squares & Mean Square & F Ratio \\
Model & 7 & 10.330228 & 1.47575 & 34.8553 \\
Error & 17 & 0.719766 & 0.04234 & Prob > F \\
C. Total & 24 & 11.049994 & & $<.0001^{*}$
\end{tabular}

\section{Parameter Estimates}

\begin{tabular}{lrrrrrr}
\hline Term & Estimate & Std Error & t Ratio & Prob $>|\mathbf{t}|$ & Lower 95\% & Upper 95\% \\
Intercept & 8.9094286 & 0.077772 & 114.56 & $<.0001^{*}$ & 8.7453443 & 9.0735128 \\
A & -0.7375 & 0.048499 & -15.21 & $<.0001^{*}$ & -0.839824 & -0.635176 \\
B & -0.088 & 0.048499 & -1.81 & 0.0873 & -0.190324 & 0.0143245 \\
C & 0.0321111 & 0.048499 & 0.66 & 0.5168 & -0.070213 & 0.1344356 \\
D & 0.0633333 & 0.048499 & 1.31 & 0.2090 & -0.038991 & 0.1656578 \\
$B^{\star} \mathrm{C}$ & 0.0464375 & 0.051441 & 0.90 & 0.3793 & -0.062094 & 0.154969 \\
A $^{\star} \mathrm{D}$ & -0.084187 & 0.051441 & -1.64 & 0.1201 & -0.192719 & 0.024344 \\
$\mathrm{C}^{\star} \mathrm{C}$ & -0.179206 & 0.091655 & -1.96 & 0.0672 & -0.372581 & 0.0141688
\end{tabular}


All Regions $90^{\text {th }}$ Percentile Stress Desirability:

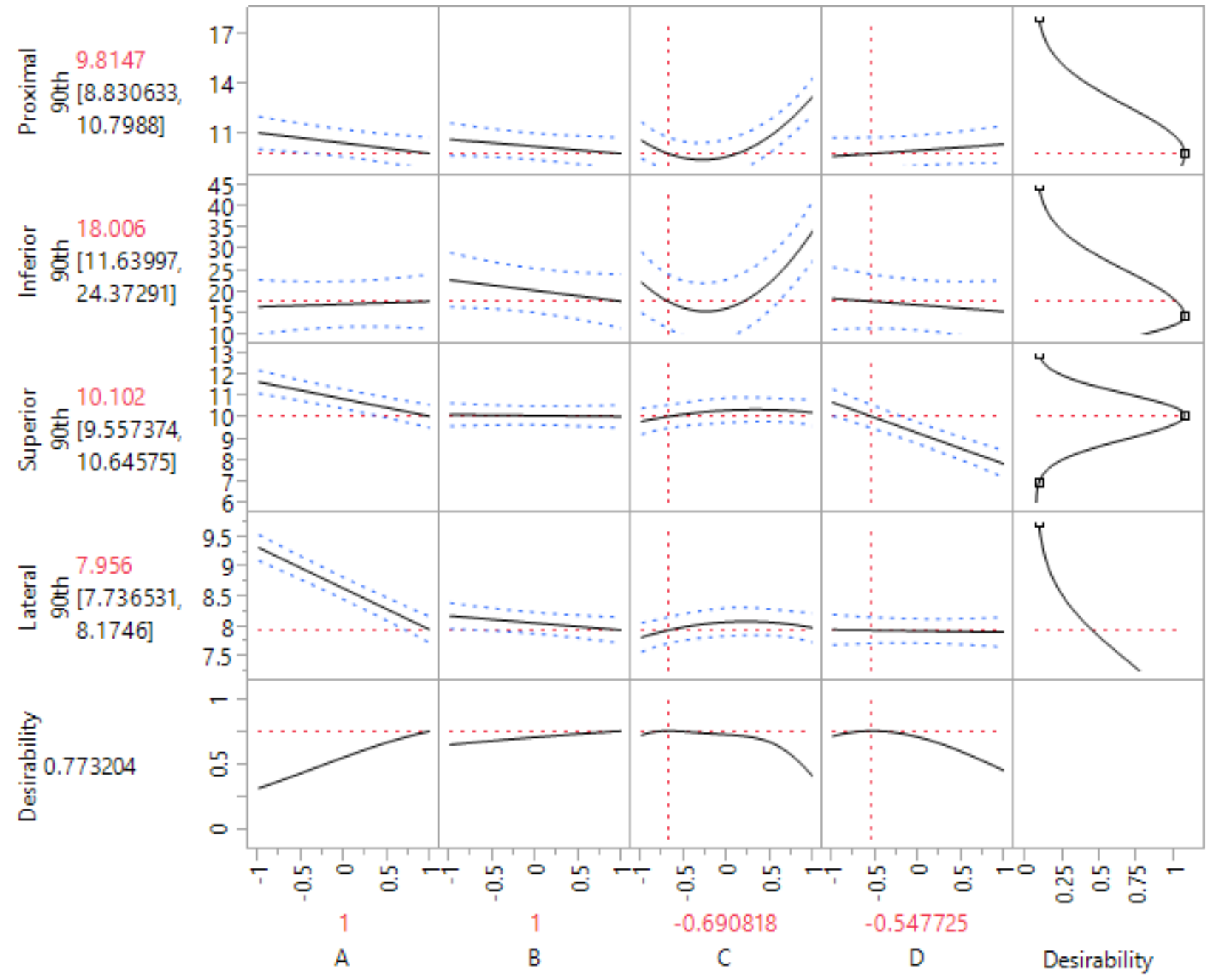


Method 2 - Proximal Region \% of Intact:

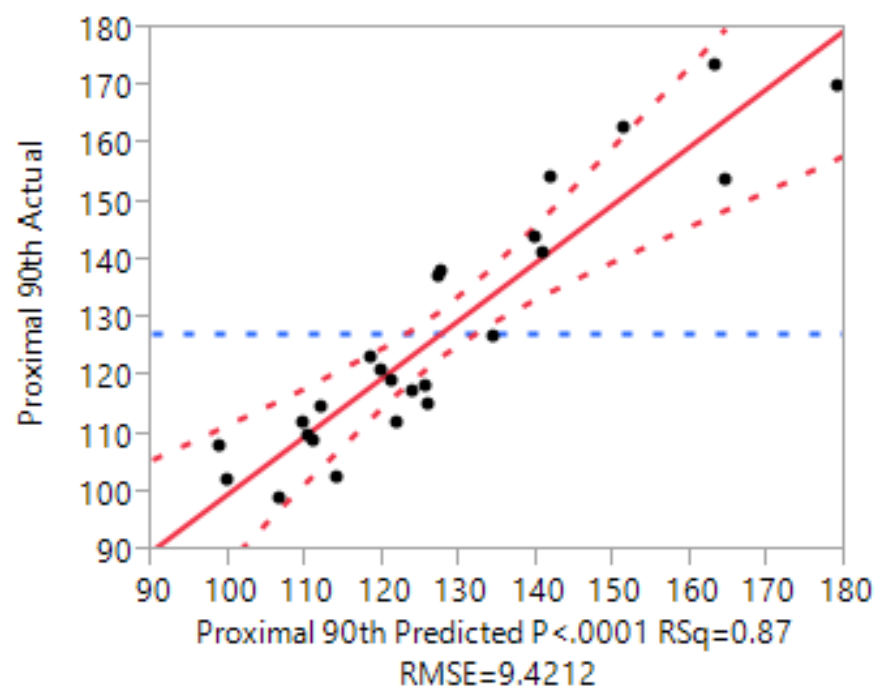

\begin{tabular}{l|r|}
\hline Summary of Fit & \\
\hline RSquare & 0.868494 \\
RSquare Adj & 0.814344 \\
Root Mean Square Error & 9.421224 \\
Mean of Response & 127.459 \\
\hline Observations (or Sum Wgts) & 25 \\
\hline
\end{tabular}

\section{Analysis of Variance}

\begin{tabular}{lrrrrr} 
& \multicolumn{5}{c}{ Sum of } \\
Source & DF & Squares & Mean Square & F Ratio \\
Model & 7 & 9965.162 & 1423.59 & 16.0388 \\
Error & 17 & 1508.911 & 88.76 & Prob $>$ F \\
C. Total & 24 & 11474.073 & & $<.0001^{*}$
\end{tabular}

\section{Parameter Estimates}

\begin{tabular}{lrrrrrr}
\hline Term & Estimate & Std Error & t Ratio & Prob $>|\mathbf{t}|$ & Lower 95\% & Upper 95\% \\
Intercept & 110.35179 & 3.560888 & 30.99 & $<.0001^{*}$ & 102.83897 & 117.86461 \\
A & -10.69962 & 2.220604 & -4.82 & $0.0002^{*}$ & -15.38468 & -6.014554 \\
B & 0.6684413 & 2.220604 & 0.30 & 0.7671 & -4.016623 & 5.3535059 \\
C & 6.5917684 & 2.220604 & 2.97 & $0.0086^{*}$ & 1.9067038 & 11.276833 \\
D & 11.619217 & 2.220604 & 5.23 & $<.0001^{*}$ & 6.9341525 & 16.304282 \\
B $^{\star} \mathrm{C}$ & 7.288236 & 2.355306 & 3.09 & $0.0066^{*}$ & 2.3189746 & 12.257497 \\
A $^{\star} \mathrm{D}$ & -7.862256 & 2.355306 & -3.34 & $0.0039^{*}$ & -12.83152 & -2.892995 \\
C $^{\star} \mathrm{C}$ & 23.760017 & 4.196547 & 5.66 & $<.0001^{*}$ & 14.906077 & 32.613957
\end{tabular}


Method 2 - Inferior Region \% of Intact:

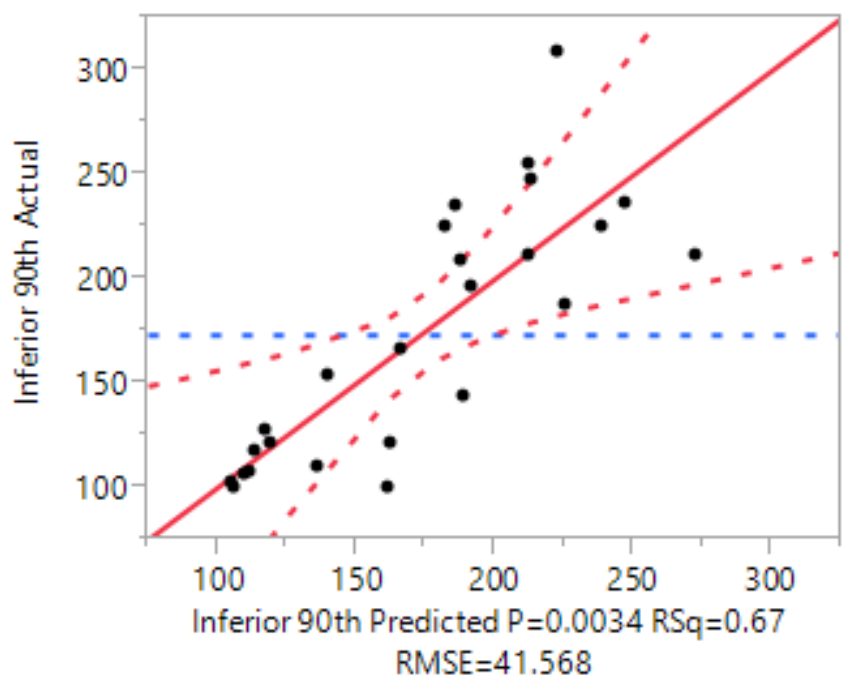

\begin{tabular}{l|r}
\hline Summary of Fit & \\
\hline RSquare & 0.670702 \\
RSquare Adj & 0.535108 \\
Root Mean Square Error & 41.56827 \\
Mean of Response & 173.0702 \\
\hline Observations (or Sum Wgts) & 25 \\
\hline
\end{tabular}

Analysis of Variance

\begin{tabular}{lrrrrr} 
& \multicolumn{5}{c}{ Sum of } \\
Source & DF & Squares & Mean Square & F Ratio \\
Model & 7 & 59829.117 & 8547.02 & 4.9464 \\
Error & 17 & 29374.660 & 1727.92 & Prob > F \\
C. Total & 24 & 89203.777 & & $0.0034^{*}$
\end{tabular}

\section{Parameter Estimates}

$\begin{array}{lrrrrrr}\text { Term } & \text { Estimate } & \text { Std Error } & \text { t Ratio } & \text { Prob }>\mid \mathbf{t |} & \text { Lower 95\% } & \text { Upper 95\% } \\ \text { Intercept } & 111.72064 & 15.71133 & 7.11 & <.0001^{*} & 78.572636 & 144.86865 \\ \text { A } & -5.323608 & 9.797735 & -0.54 & 0.5939 & -25.99502 & 15.347807 \\ \text { B } & 1.9240838 & 9.797735 & 0.20 & 0.8466 & -18.74733 & 22.595499 \\ \text { C } & 15.051911 & 9.797735 & 1.54 & 0.1429 & -5.619504 & 35.723326 \\ \text { D } & 7.0838565 & 9.797735 & 0.72 & 0.4795 & -13.58756 & 27.755271 \\ B^{\star} \mathrm{C} & 28.016401 & 10.39207 & 2.70 & 0.0153^{\star} & 6.0910546 & 49.941747 \\ \text { A }^{\star} \mathrm{D} & -17.88872 & 10.39207 & -1.72 & 0.1033 & -39.81407 & 4.036623 \\ \mathrm{C}^{\star} \mathrm{C} & 85.207733 & 18.51598 & 4.60 & 0.0003^{*} & 46.142431 & 124.27304\end{array}$




\section{Method 2 - Superior Region \% of Intact:}

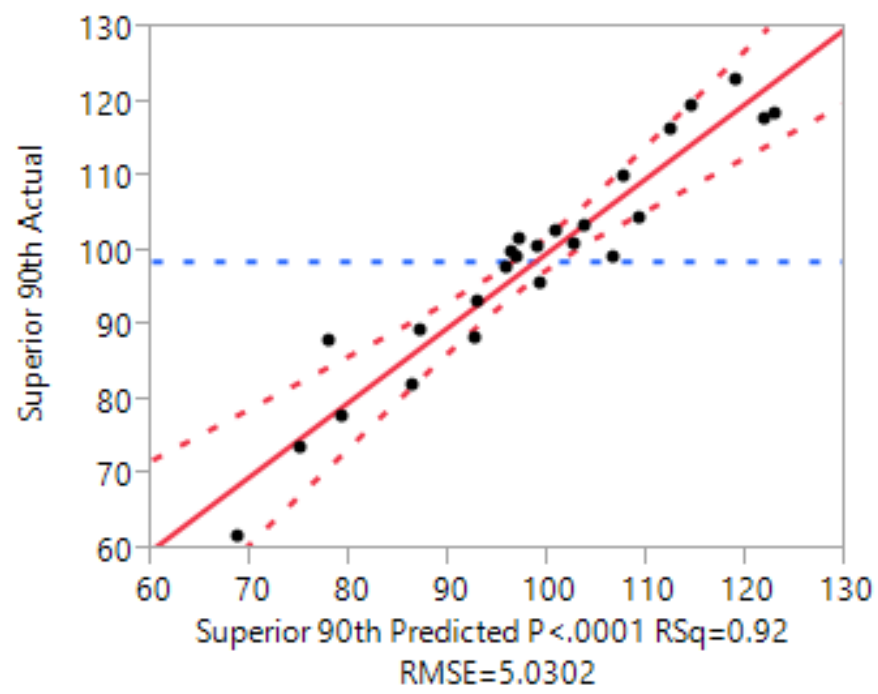

\begin{tabular}{|c|c|c|c|c|}
\hline \multicolumn{4}{|c|}{ Summary of Fit } & \\
\hline \multicolumn{3}{|l|}{ RSquare } & 0.919737 & \\
\hline \multicolumn{3}{|c|}{ RSquare Adj } & 0.886687 & \\
\hline \multicolumn{3}{|c|}{ Root Mean Square Error } & 5.030196 & \\
\hline \multicolumn{3}{|c|}{ Mean of Response } & 98.60017 & \\
\hline \multicolumn{3}{|c|}{ Observations (or Sum Wgts) } & 25 & \\
\hline \multicolumn{5}{|c|}{ Analysis of Variance } \\
\hline \multirow{2}{*}{ Source } & DF & $\begin{array}{r}\text { Sum of } \\
\text { Squares }\end{array}$ & Mean Square & F Ratio \\
\hline & 7 & 4929.0606 & 704.152 & 27.8289 \\
\hline Error & 17 & 430.1488 & 25.303 & Prob $>F$ \\
\hline C. Total & 24 & 5359.2095 & & $<.0001^{*}$ \\
\hline
\end{tabular}

\section{Parameter Estimates}

\begin{tabular}{lrrrrrr} 
Term & Estimate & Std Error & t Ratio & Prob $>|\mathbf{t}|$ & Lower 95\% & Upper 95\% \\
\hline Intercept & 100.82011 & 1.901235 & 53.03 & $<.0001^{*}$ & 96.808858 & 104.83137 \\
A & -8.284487 & 1.185629 & -6.99 & $<.0001^{*}$ & -10.78594 & -5.783029 \\
B & 1.8935374 & 1.185629 & 1.60 & 0.1287 & -0.60792 & 4.3949951 \\
C & -1.362181 & 1.185629 & -1.15 & 0.2665 & -3.863639 & 1.1392768 \\
D & -13.6663 & 1.185629 & -11.53 & $<.0001^{*}$ & -16.16776 & -11.16485 \\
$B^{\star} \mathrm{C}$ & 3.3575624 & 1.257549 & 2.67 & $0.0162^{*}$ & 0.7043659 & 6.010759 \\
$A^{*} D$ & -0.593072 & 1.257549 & -0.47 & 0.6432 & -3.246268 & 2.0601248 \\
$C^{*} \mathrm{C}$ & -3.083251 & 2.240627 & -1.38 & 0.1867 & -7.810561 & 1.64406
\end{tabular}




\section{Method 2 - Lateral Region \% of Intact:}

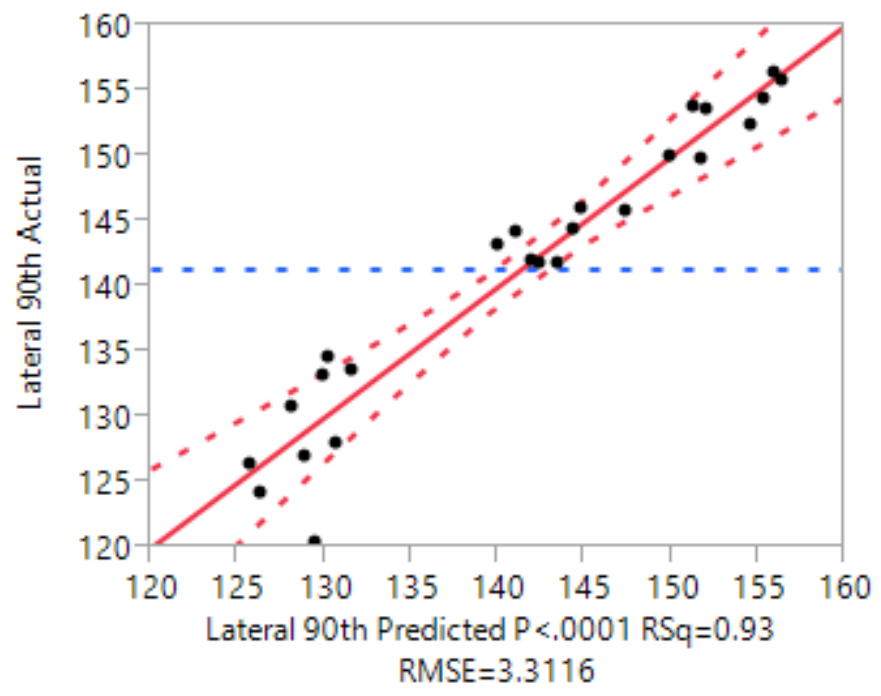

\section{Summary of Fit}

\begin{tabular}{lr}
\hline RSquare & 0.934869 \\
RSquare Adj & 0.90805 \\
Root Mean Square Error & 3.311608 \\
Mean of Response & 141.3405 \\
Observations (or Sum Wgts) & 25
\end{tabular}

Analysis of Variance

\begin{tabular}{lrrrrr} 
& & Sum of & & \\
Source & DF & Squares & Mean Square & F Ratio \\
\hline Model & 7 & 2676.0113 & 382.287 & 34.8588 \\
\hline Error & 17 & 186.4348 & 10.967 & Prob > F \\
C. Total & 24 & 2862.4461 & & $<.0001^{*}$
\end{tabular}

\section{Parameter Estimates}

$\begin{array}{lrrrrrr}\text { Term } & \text { Estimate } & \text { Std Error } & \text { t Ratio } & \text { Prob }>|\mathbf{t}| & \text { Lower 95\% } & \text { Upper 95\% } \\ \text { Intercept } & 143.41829 & 1.25167 & 114.58 & <.0001^{*} & 140.7775 & 146.05909 \\ \text { A } & -11.87025 & 0.780554 & -15.21 & <.0001^{*} & -13.51707 & -10.22342 \\ \text { B } & -1.416084 & 0.780554 & -1.81 & 0.0873 & -3.062908 & 0.2307398 \\ \text { C } & 0.5170974 & 0.780554 & 0.66 & 0.5165 & -1.129727 & 2.1639216 \\ \text { D } & 1.0174805 & 0.780554 & 1.30 & 0.2098 & -0.629344 & 2.6643047 \\ B^{\star} \mathrm{C} & 0.7455946 & 0.827902 & 0.90 & 0.3804 & -1.001126 & 2.4923154 \\ A^{\star} \mathrm{D} & -1.354433 & 0.827902 & -1.64 & 0.1202 & -3.101154 & 0.3922878 \\ \mathrm{C}^{\star} \mathrm{C} & -2.885767 & 1.475108 & -1.96 & 0.0671 & -5.997972 & 0.2264377\end{array}$


All Regions $90^{\text {th }}$ Percentile \% of Intact Desirability:

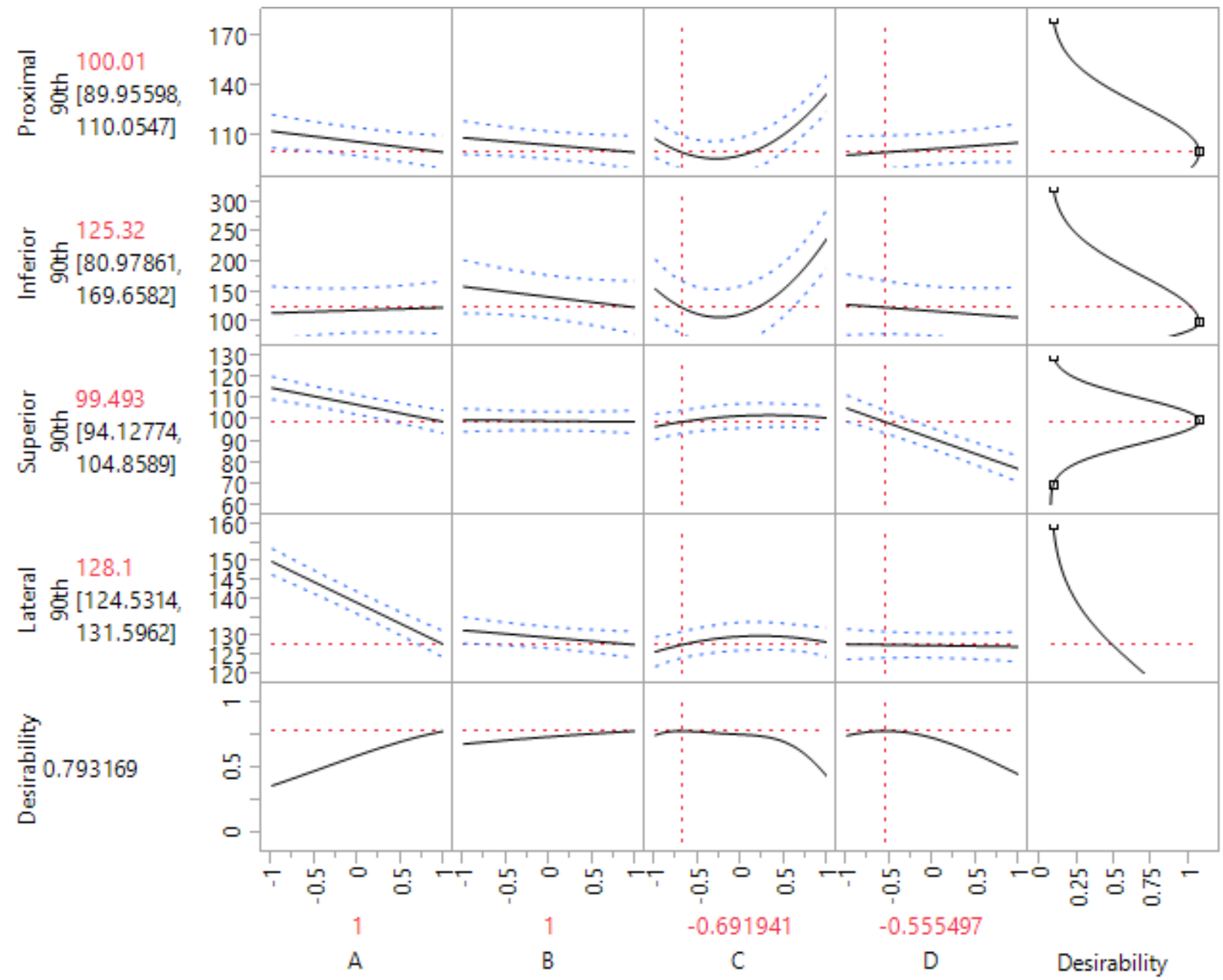

